\title{
₹USGS
}

\section{HAWAIIAN VOLCANO OBSERVATORY}

1963 QUARTERLY ADMINISTRATIVE REPORTS

INTRODUCTORY NOTE BY THOMAS L. WRIGHT AND JENNIFER S. NAKATA

\section{COMPILED BY JENNIFER S. NAKATA}

\author{
SUMMARY 29 \\ JANUARY, FEBRUARY, AND MARCH 1963 \\ By RoBERT Y. KoYAnAgi, ARnOLd T. OKAMURA, \\ harold L. KRIVOY, AND AKIRA Yamamoto
}

\section{SUMMARY 30}

APRIL, MAY, AND JUNE 1963

BY HAROLD L. KRIVOY, WiLLIE T. KinOSHITA, ARNOLD T. OKAMURA, AND ROBERT Y. KOYANAGI

SUMMARY 31

July, August, ANd SEPTEMBER 1963

By RoBERT Y. KOYANAgi, ARNOLd T. OKAMURA,

Willie T. Kinoshita, James G. MoOre, and Howard A. PoWers

SUMMARY 32

OCTOBER, NOVEMBER, AND DECEMBER 1963

By ARnold T. OKAMura, Robert Y. Koyanagi, Willie T. Kinoshita, James G. MoOre, DAllas L. PECK, ANd HoWARd A. POWERS

OPEN-FILE REPORT 2007-1323

U.S. DEPARTMENT OF THE INTERIOR

U.S. GEOLOGICAL SURVEY 


\title{
U.S. Department of the Interior DIRK KEMPTHORNE, Secretary
}

\author{
U.S. Geological Survey \\ Mark D. Myers, Director
}

U.S. Geological Survey, Reston, Virginia 2007

For product and ordering information:

World Wide Web: http://www.usgs.gov/pubprod

Telephone: 1-888-ASK-USGS

For more information on the USGS - the Federal source for science about the Earth, its natural and living resources, natural hazards, and the environment:

World Wide Web: http://www.usgs.gov

Telephone: 1-888-ASK-USGS

Any use of trade, product, or firm names is for descriptive purposes only and does not imply endorsement by the U.S. Government.

Although this report is in the public domain, permission must be secured from the individual copyright owners to reproduce any copyrighted material contained within this report. 


\section{INTRODUCTORY NOTE}

The Hawaiian Volcano Observatory Summaries have been published in the current format since 1956. The Quarterly Summaries (1956 through 1973) and the Annual Summaries (1974 through 1985) were originally published as Administrative Reports. These reports have been compiled and published as U.S. Geological Survey Open-File Reports. The quarterly reports have been combined and published as one annual summary. All the summaries from 1956 to the present are now available as .pdf files at http://www.usgs.gov/pubprod.

The earthquake summary data are presented as a listing of origin time, depth, magnitude, and other location parameters. Network instrumentation, field station sites, and location algorithms are described. Tilt and other deformation data are included until Summary 77, January to December 1977. From 1978, the seismic and deformation data are published separately, due to differing schedules of data reduction.

There are eight quarters - from the fourth quarter of 1959 to the third quarter of 1961 — that were never published. Two of these ( $4^{\text {th }}$ quarter $1959,1^{\text {st }}$ quarter 1960) have now been published, using handwritten notes of Jerry Eaton (HVO seismologist at the time) and his colleagues. The seismic records for the remaining six summaries went back to California in 1961 with Jerry Eaton. Other responsibilities intervened, and the seismic summaries were never prepared.

\section{Chronology}

The following Kìlauea eruption chronology covers the two recent reports and the six missing quarters:

\begin{tabular}{|l|l|l|l|}
\hline Location & Beginning Date & Ending Date & Comment \\
\hline Kìlauea Iki crater (Kîlauea's summit) & $11 / 14 / 1959$ & $12 / 20 / 1959$ & 19 eruptive episodes \\
\hline Kapoho (lower east rift zone) & $1 / 13 / 1960$ & $2 / 18 / 1960$ & 4 eruption stages \\
\hline Halemaumau (Kīlauea's summit) & $2 / 24 / 1961$ & $2 / 24 / 1961$ & $\begin{array}{l}\text { Intermittent activity during } \\
\text { uninterrupted inflation fol- } \\
\text { lowing the 1960 eruption }\end{array}$ \\
\hline Halemaumau (Kīlauea's summit) & $3 / 22 / 1961$ & $3 / 25 / 1961$ & Same as above. \\
\hline Halemaumau (Kīlauea's summit) & $7 / 10 / 1961$ & $7 / 17 / 1961$ & Same as above. \\
\hline Heiheiahulu (middle east rift zone) & $9 / 22 / 1961$ & $9 / 25 / 1961$ & $\begin{array}{l}\text { First historical east rift erup- } \\
\text { tion at this location }\end{array}$ \\
\hline
\end{tabular}

The 1959-1960 eruptions were among two of the most spectacular Kilauea eruptions. The HVO staff was kept busy with acquisition of unusually high quantities of instrumental data and observations of the two sequences, which were separated by less than one month. Even with a year's interval before the beginning of the summit-east rift sequence in 1961, the staff never caught up, and the seismic records were set aside for later study.

A total of 1,672 earthquakes-1,106 for 1960 and 566 for 1961-are part of HVO's cataloged database. The annual listings have been appended to the $1^{\text {st }}$ Quarter Report of 1960 and to the $4^{\text {th }}$ Quarter Report for 1961 . The number of earthquakes is probably low, biased toward the larger magnitudes. The entire HVO catalog, including 1960 and 1961, is accessible from the ANSS CATALOG SEARCH site at http://www.ncedc.org/anss/catalog-search. 
UNITED STATES

DEPARTMENT OF THE INTERIOR

GEOLOGICAL SURVEY

\section{HAWAIIAN VOLCANO OBSERVATORY \\ SUMMARY 29 \\ January, February, and March, 1963}

By

Robert Y. Koyanagi, Arnold T. Okamura, Harold L. Krivoy, and Akira Yamamoto

Issued September 1964

Observatory Staff

Geology:

Geochemistry :

J. G. Moore (Scientist-in-Charge)

C. K. Wentworth

R. T. Okamura

Geophysics:

Support:

J. C. Forbes

G. Kojima

W. H. Francis

R. Y. Koyanagi

B. J. Loucks

H. L. Krivoy

N. Sherrill

A. T. Okamura

A. Yamamoto

A. S. Ryall

Japan-U.S. Cooperative Program

E. T. Endo 
Chronological summary-1.

Tilting of the ground around Kilauea caldera-...... 4

Seismic summary-1 8

Persons or agencies reporting felt earthquakes during the quarter--- 36 Illustrations

Figure 1. Map of the island of Hawaii showing seismograph stations and localities mentioned in the text............... 3

2. Tilting of the ground around Kilauea caldera-.......- 7

\section{Tables}

Table 1. Tilt coordinates at Uwekahuna vault-_... 5

2. Tilt coordinates and changes at bases around Kilauea

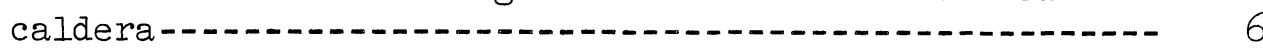

3. Numbers of earthquakes and minutes of tremor recorded on seismographs around Kilauea caldera-......... 9

4. Local earthquakes- 14

5. Distant earthquakes

6. U.S. Geological Survey seismograph stations in Hawaii--- 33 
Chronological summary

Following its small, sharp subsidence during the December 1962 Aloi eruption, the summit region of Kilauea promptly resumed the cycle of swelling that began in the summer of 1960. A moderate rate of inflation during the first quarter of 1963 is indicated by the average rate of outward tilting at the inner ring of tilt bases (Uwe, TM, Kea, and Kam), which was 11 microradians per month between December 18, 1962, and March 18, 1963. As was true throughout 1962, tilting at Kamokukolau was more rapid than at any other base during this interval.

The average daily count of shallow Kilauea caldera earthquakes diminished from 113 during the first half of January, to 62 during the second half, and to 50 during the first half of February. A moderate resurgence of these earthquakes during the second half of February sent the average daily count to 138. The level of this activity remained moderately high throughout March, with an average of 92 per day.

The frequency of earthquakes from the southwest rift zone of Kilauea and the nearby Kaoiki fault system rose from an average of 6 per day during January to 15 per day during February and March.

The number of earthquakes from the upper part of the east rift zone of Kilauea (near Aloi Crater) also increased during the quarter, from a total of 20 in January, to 49 in February, and to 92 in March. These quakes were particularly frequent late in March, when 83 occurred between the 19th and 27 th.

Deep tremor was quite prominent during the quarter with totals of 82 minutes during January, 75 minutes during February, and 119 minutes during March.

Excluding January 8 and 9, the frequency of earthquakes from the source about $30 \mathrm{~km}$ beneath Halemaumau was about 5 per day during the entire quarter. A flurry of activity from this source on January 8 and 9, however, produced 154 earthquakes. Four of these were felt (table 4, p. 14 and 15 ).

The first of these earthquakes--which occurred on January 8, at 09 h $39^{m} 44.9^{5}$, magnitude 4.3 , was the largest earthquake in Hawaii during January and was felt throughout Hawaii and on Maui and Oahu.

The lower part of Kilauea's east rift zone near Pahoa was the source of occasional earthquakes throughout the quarter and of a mild swarm of 55 earthquakes during the first week in March. Five earthquakes from this region were felt in Pahoa (table 4, p. 16, 17, and 18).

The largest earthquake in the Hawaiian region during February was not felt. It originated $83 \mathrm{~km}$ west of Keahole Point at $09^{\mathrm{h}} 08^{\mathrm{m}}$ on the 17 th and had a magnitude of 4.1 . 
During March, 6 earthquakes in the Hawaiian Islands had magnitudes of 3.5 or greater (table 4, p. 18 and 19).

The largest earthquake in March, with a magnitude of 4.5 , originated $14 \mathrm{~km}$ southeast of Waikii at a depth of about $13 \mathrm{~km}$ at $22^{\mathrm{h}} 32^{\mathrm{m}}$ on the $24 \mathrm{th}$. It was felt over all of the island of Hawaii and on Maui.

A very interesting earthquake was felt throughout Maui at $07^{\mathrm{h}_{1}} 9^{\mathrm{m}}$ on March 25. It had a magnitude of 3.9 and originated $35 \mathrm{~km}$ beneath Haleakala National Park headquarters. There were neither foreshocks nor aftershocks.

other earthquakes felt during the quarter are listed in table 4. 


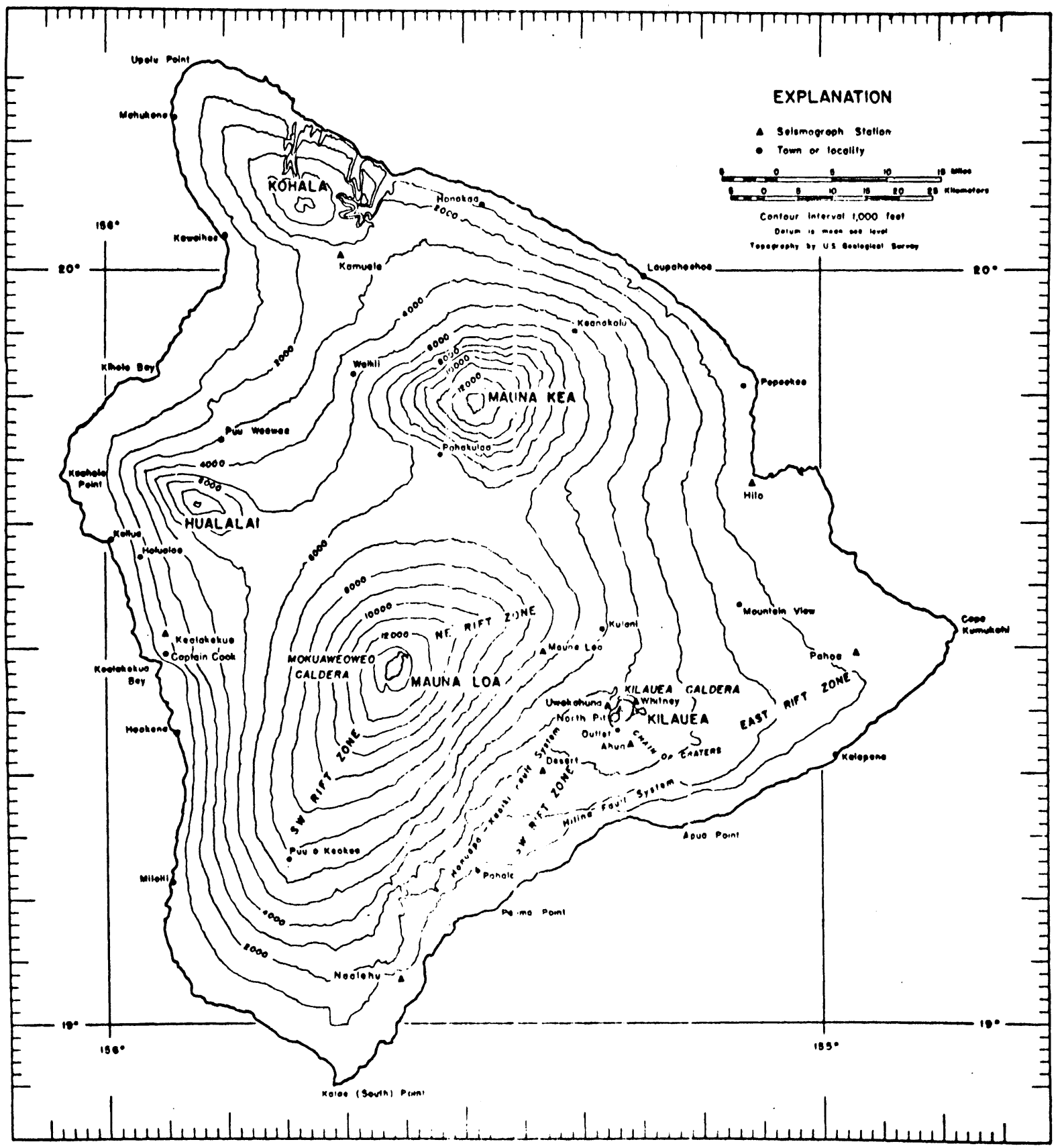

Figure 1.--Map of the island of Hawail showing selsmograph stations operated by the Geological Survey and localities mentioned in the text. Epicenters of local earthquakes are given in terms of geographic coordinates, which are indicated at the edges of the map. 
Tilting of the ground around Kilauea caldera.--Tilting of the ground around the sumit of Kilauea is monitored daily by a shortabase water-tube tiltmeter in Uwekahuna Vault (table 1), and at irregular intervals it is measured on a regional scale by means of a network of field tilt bases and a portable water-tube tiltmeter (table 2). The attitude of the ground surface at each tilt base is reported in terms of north-south and east-west tilt coordinates. Both coordinates at each station were arbitrarily set equal to 500 when measurements at that station were begun. Increasing tilt coordinates correspond to northward anci eastward tilting of the earth's surface, i.e., to a relative subsidence toward the north and east. A one-unit change in coordinate cooresponds to a tilting of 1 microradian ( $1 \mathrm{~mm}$ per $\mathrm{km}$ ) in the direction indicated. 
Table 1.--Tilt coordinates Uwekahuna Vault, January,

February, and March, 1963

\begin{tabular}{|c|c|c|c|c|c|}
\hline Date & $\mathrm{N}-\mathrm{S}$ & $E-W$ & Date & $\mathrm{N}-\mathrm{S}$ & $E-W$ \\
\hline $\operatorname{Jan} .6$ & 460 & 492 & Mar. 3 & 470 & 486 \\
\hline 13 & 465 & 492 & 10 & 476 & 482 \\
\hline 20 & 467 & 490 & 17 & 485 & 482 \\
\hline 27 & 467 & 490 & 24 & 488 & 482 \\
\hline Feb. 3 & 467 & 490 & 31 & 489 & 483 \\
\hline 10 & 468 & 491 & & & \\
\hline 17 & 470 & 487 & & & \\
\hline 24 & 469 & 488 & & & \\
\hline
\end{tabular}


Table 2.--Tilt coordinates and changes at bases around Kilauea caldera,

Ist Quarter, 1963 (see fig. 2)

\begin{tabular}{|c|c|c|c|c|c|c|}
\hline \multirow{2}{*}{$\begin{array}{l}\text { Tilt base } \\
\text { (location) }\end{array}$} & \multirow{2}{*}{$\begin{array}{l}\text { Date } \\
(1963)\end{array}$} & \multicolumn{2}{|c|}{ Tilt coordinates } & \multirow{2}{*}{\multicolumn{2}{|c|}{$\begin{array}{l}\text { Rate }\left(10^{-6} \mathrm{rad} / \mathrm{mo}\right) \\
\text { and direction of } \\
\text { tilting since } \\
\text { last reading }\end{array}$}} & \multirow{2}{*}{$\begin{array}{c}\text { Date } \\
\text { last reading } \\
(1962)\end{array}$} \\
\hline & & $\mathrm{N}-\mathrm{S}$ & $E-W$ & & & \\
\hline $\begin{array}{l}\text { Uwekahuna } \\
\left(19^{\circ} 25.5^{\prime} \mathrm{N} ., 155^{\circ} 17.4^{\prime} \mathrm{W} .\right)\end{array}$ & Mar. 20 & 471.2 & 481.2 & 11.6 & N. $28.5^{\circ} \mathrm{W}$. & Dec. 10 \\
\hline $\begin{array}{l}\text { Tree Molds } \\
\left(19^{\circ} 26.3^{\prime} \text { N., } 155^{\circ} 17.3^{\prime} \text { W. }\right)\end{array}$ & Mar. 19 & 448.7 & 511.0 & 4.0 & N. $6.9^{\circ} \mathrm{W}$. & Dec. 12 \\
\hline $\begin{array}{l}\text { Sand Spit } \\
\left(19^{\circ} 24.1^{\prime} \mathrm{N} ., 155^{\circ} 16.8^{\prime} \mathrm{W} .\right)\end{array}$ & Mar. 21 & 938.5 & 707.5 & $13 \cdot 9$ & N. $6.0^{\circ} \mathrm{E}$. & Dec. 12 \\
\hline $\begin{array}{l}\text { Kalihipaa } \\
\left(19^{\circ} 21.4^{\prime} \mathrm{N} ., 155^{\circ} 15.3^{\prime} \mathrm{W} .\right)\end{array}$ & Mar. 18 & 568.5 & $428 \cdot 3$ & 2.6 & $\mathrm{~S} .33 .0^{\circ} \mathrm{E}$. & Dec. 11 \\
\hline $\begin{array}{l}\text { Keamoku } \\
\left(19^{\circ} 25.1^{\prime} \mathrm{N}^{\prime}, 155^{\circ} 19.0^{\prime} \mathrm{W} .\right)\end{array}$ & Mar. 18 & $494 \cdot 5$ & 577.6 & 10.7 & N. $54.1^{\circ} \mathrm{W}$. & Dec. II \\
\hline $\begin{array}{l}\text { Ahua Kamokukolau } \\
\left(19^{\circ} 22.7^{\prime} \text { N., } 155^{\circ} 16.6^{\prime} \text { W. }\right)\end{array}$ & Mar. 19 & $599 \cdot 3$ & 539.3 & 18.5 & S. $16.7^{\circ} \mathrm{E}$. & Dec. 10 \\
\hline $\begin{array}{l}\text { Kipuka Nene } \\
\left(19^{\circ} 19.4^{\prime} \text { W., } 155^{\circ} 16.7^{\prime} \mathrm{W} \text {. }\right)\end{array}$ & Mar. 15 & 507.1 & 496.7 & 1.1 & S. $5^{\circ} \mathrm{E}$. & Dec. 13 \\
\hline $\begin{array}{l}\text { Hilina Pali } \\
\left(19^{\circ} 18.2^{\prime} \mathrm{N} ., 155^{\circ} 18.6^{\prime} \mathrm{W} .\right)\end{array}$ & Mar. 13 & 509.6 & 497.4 & 0.6 & S. $27.6^{\circ} \mathrm{E}$. & oct. 24 \\
\hline $\begin{array}{l}\text { Kapapala Ranch } \\
\left(19^{\circ} 20.5^{\prime} \text { N., } 155^{\circ} 23.8^{\prime} \text { W. }\right)\end{array}$ & Mar. 14 & 497.4 & 505.0 & 0.1 & S. $27^{\circ} \mathrm{E}$. & Dec. 19 \\
\hline
\end{tabular}




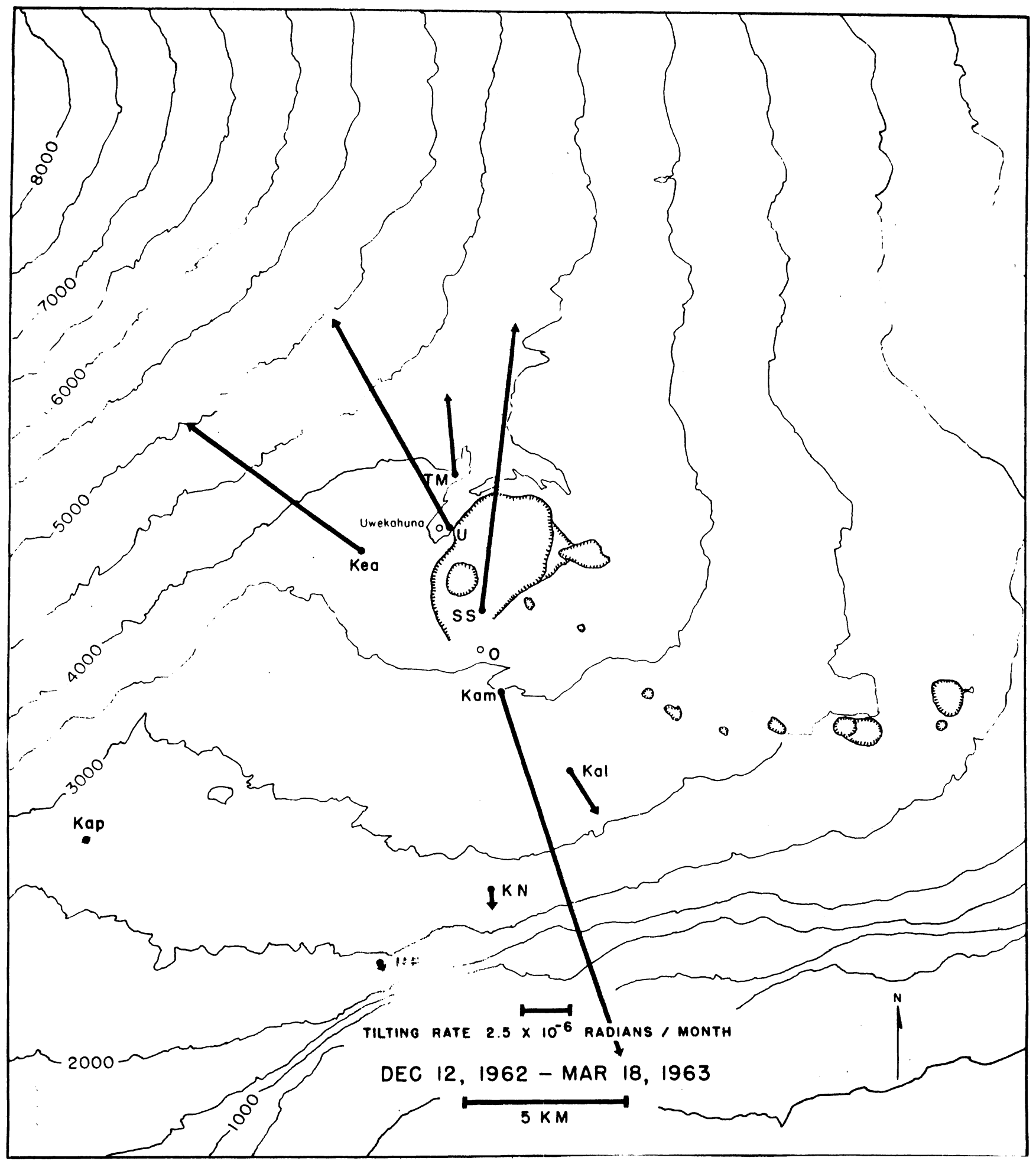

Figure 2.--Tilting of the ground around Kilauea caldera. The vector depicting tilting at a given base points in the direction of maximum relative subsidence and has a length proportional to the rate of tilting during the measurement interval. 
Seismic summary.--Events recorded by the U.S. Geological Survey seismograph network in Hawail fall into two categories: local earthquakes and tremor originating in the region of the Hawaiian Islands, usually within $100 \mathrm{~km}$ of at least one seismograph, and distant earthquakes originating more than $3,000 \mathrm{~km}$ from Hawail. As an index of seismic activity at Hawaiian volcanoes, daily counts of earthquakes and minutes of tremor recorded by seismographs in Hawaii are listed in table 3. The earthquakes are separated into groups on the basis of region of origin as determined primarily by analysis of records obtained daily at the Observatory (U, M, A, D, N). Earthquakes of magnitude 2.5 or greater are generally sufficiently well recorded to be located with greater precision; they are listed individually in table 4. Data on identifiable phases from distant earthquakes are listed in table 5.

Locations of the seismograph stations are shown on figure 1. Essential data on the stations are given in table 6. 
Table 3.--Numbers of earthquakes and minutes of tremor recorded on

\section{seismographs $\mathrm{U}, \mathrm{M}, \mathrm{A}, \mathrm{D}$, and $\mathrm{N}$ around Kilauea caldera}

Tremor is separated into three categories: deep, intermediate, and shallow, on the basis of relative amplitudes on seismographs in the sumit region. Unless otherwise stated, tremor is presumed to be associated with movement of magma within the central complex of Kilauea.

Earthquake categories are: Halemaumau rock slides, which are detected by the characteristic record they produce on the North Pit seismograph; shallow earthquakes in the Kilauea caldera region; shallow earthquakes along the SW. rift zone of Kilauea and the adjacent portion of the Kaoiki fault system; earthquakes along the eastern half of Kilauea's east rift zone (from the Pahoa seismograph); earthquakes from a source about $30 \mathrm{~km}$ beneath Halemaumau; earthquakes from the upper east rift zone and the adjacent fault systems on Kilauea's south flank, and earthquakes from other regions: Kona, Mauna Kea, etc.

\begin{tabular}{|c|c|c|c|c|c|c|c|c|c|c|}
\hline \multirow{2}{*}{$\begin{array}{l}\text { Date } \\
(1963)\end{array}$} & & $\begin{array}{l}\text { Tremor } \\
\text { in minute }\end{array}$ & & \multicolumn{7}{|c|}{ Earthquakes } \\
\hline & Deep & $\begin{array}{l}\text { Inter- } \\
\text { mediate }\end{array}$ & Shallow & $\begin{array}{l}\text { Hale- } \\
\text { maumau } \\
\text { slides }\end{array}$ & $\begin{array}{l}\text { Kilauea } \\
\text { Caldera }\end{array}$ & $\begin{array}{c}\text { SW. rift } \\
\text { and } \\
\text { Kaoiki }\end{array}$ & $\begin{array}{c}\text { Eastern } \\
\text { East } \\
\text { rift }\end{array}$ & $\begin{array}{l}\text { Hale- } \\
\text { maumau } \\
30 \mathrm{~km}\end{array}$ & $\begin{array}{l}\text { Kala- } \\
\text { pana } \\
\text { Trail }\end{array}$ & Others \\
\hline $\begin{array}{r}\operatorname{Jan} .1 \\
2 \\
3 \\
4 \\
5 \\
6 \\
7 \\
8 \\
9 \\
10 \\
11 \\
12 \\
13 \\
14\end{array}$ & 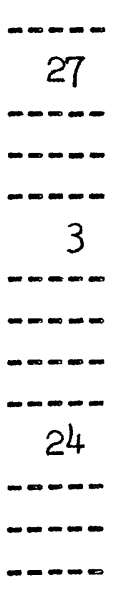 & 正 & & $\begin{array}{c}- \\
- \\
-\end{array}$ & $\begin{array}{r}105 \\
110 \\
115 \\
110 \\
110 \\
127 \\
75 \\
82 \\
160 \\
115 \\
80 \\
110 \\
107 \\
170\end{array}$ & $\begin{array}{r}9 \\
12 \\
3 \\
3 \\
4 \\
7 \\
3 \\
6 \\
4 \\
5 \\
5 \\
8 \\
5 \\
5\end{array}$ & $\begin{array}{c}- \\
- \\
- \\
-\end{array}$ & $\begin{array}{r}2 \\
1 \\
3 \\
2 \\
5 \\
4 \\
6 \\
112 \\
42 \\
5 \\
2 \\
5 \\
2 \\
4\end{array}$ & 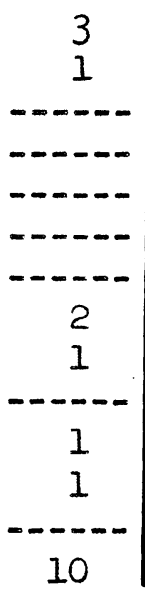 & $\begin{array}{l}1 \text { South Point } \\
1 \text { South Point } \\
1 \text { Mauna Kea } \\
1 \text { Kona } \\
1 \text { Mauna Kea } \\
1 \text { Kona } \\
1 \text { Apua Pt. } \\
1 \text { Kona } \\
-1 \text { - }\end{array}$ \\
\hline
\end{tabular}


Table 3.--Numbers of earthquakes and minutes of tremor recorded on seismographs $U_{2} M_{2} A_{2} D_{2}$ and $\mathrm{N}$ around Kilauea caldera- Continued

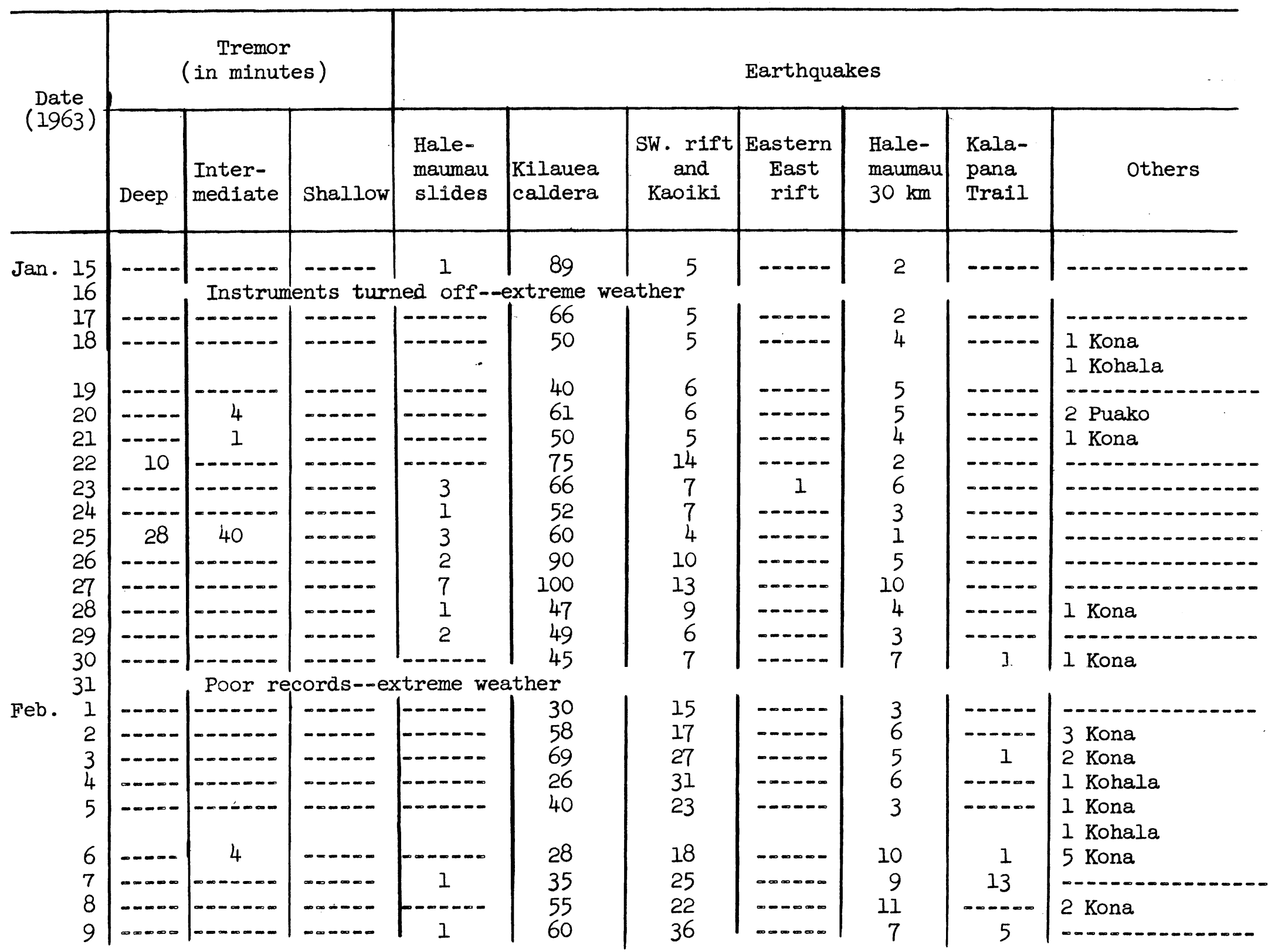


Table 3.--Numbers of earthquakes and minutes of tremor recorded on seismographs $\mathrm{U}, \mathrm{M}, \mathrm{A}, \mathrm{D}$, and $\mathrm{N}$ around Kilauea caldera--Continued

\begin{tabular}{|c|c|c|c|c|c|c|c|c|c|c|}
\hline \multirow{2}{*}{$\begin{array}{l}\text { Date } \\
(1963)\end{array}$} & \multicolumn{3}{|c|}{$\begin{array}{c}\text { Tremor } \\
\text { (in minutes) }\end{array}$} & \multicolumn{7}{|c|}{ Earthquakes } \\
\hline & Deep & $\begin{array}{l}\text { Inter- } \\
\text { mediate }\end{array}$ & Shallow & $\begin{array}{l}\text { Hale- } \\
\text { maumau } \\
\text { slides }\end{array}$ & $\begin{array}{l}\text { Kilauea } \\
\text { caldera }\end{array}$ & $\begin{array}{l}\text { SW. rift } \\
\text { and } \\
\text { Kaoiki }\end{array}$ & $\begin{array}{c}\text { Eastern } \\
\text { East } \\
\text { rift }\end{array}$ & $\begin{array}{l}\text { Hale- } \\
\text { maumau } \\
30 \mathrm{~km}\end{array}$ & $\begin{array}{l}\text { Kala- } \\
\text { pana } \\
\text { Trail }\end{array}$ & Others \\
\hline $\begin{array}{c}10 \\
11 \\
12 \\
13 \\
14 \\
15 \\
16 \\
17 \\
18 \\
19 \\
20 \\
21 \\
22 \\
23 \\
24 \\
25 \\
26 \\
27 \\
28\end{array}$ & 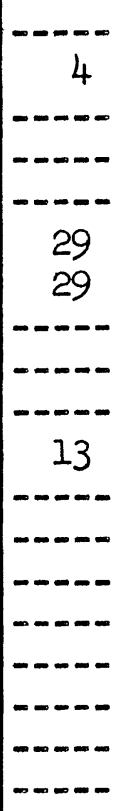 & - & - & 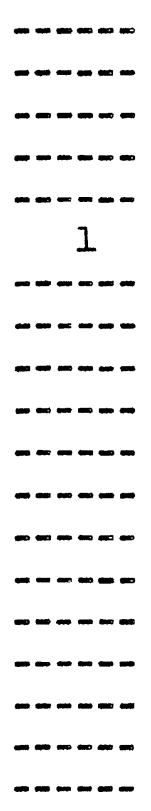 & $\begin{array}{r}50 \\
53 \\
74 \\
34 \\
40 \\
80 \\
240 \\
350 \\
300 \\
60 \\
100 \\
100 \\
45 \\
70 \\
120 \\
115 \\
150 \\
80 \\
65\end{array}$ & $\begin{array}{r}18 \\
13 \\
7 \\
7 \\
6 \\
10 \\
11 \\
10 \\
12 \\
15 \\
16 \\
8 \\
5 \\
15 \\
20 \\
14 \\
15 \\
15 \\
17\end{array}$ & 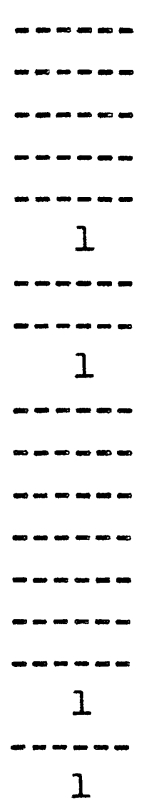 & $\begin{array}{c}4 \\
2 \\
--- \\
2 \\
2 \\
3 \\
3 \\
3 \\
13 \\
12 \\
7 \\
3 \\
5 \\
7 \\
5 \\
1 \\
2 \\
3 \\
1\end{array}$ & 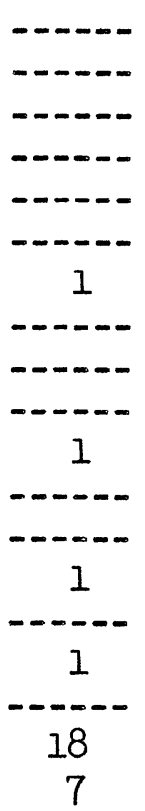 & $\begin{array}{l}3 \text { Kona } \\
1 \text { Kona } \\
1 \text { Kona } \\
1 \text { Maui } \\
1 \text { Kona } \\
4 \text { Kona } \\
1 \text { Kona } \\
1 \text { Kona } \\
1 \text { Kona } \\
1 \text { Kohala } \\
1 \text { Kona } \\
-2\end{array}$ \\
\hline
\end{tabular}


Table 3.-Numbers of earthquakes and minutes of tremor recorded on seismographs $\mathrm{V}_{2} \mathrm{M}_{5} \mathrm{~A}_{4} \mathrm{D}$, and $\mathrm{N}$ around Kilauea caldera $=$ Continued

\begin{tabular}{|c|c|c|c|c|c|c|c|c|c|c|}
\hline \multirow{2}{*}{$\begin{array}{l}\text { Date } \\
(1963)\end{array}$} & \multicolumn{3}{|c|}{$\begin{array}{c}\text { Tremor } \\
\text { (in minutes) }\end{array}$} & \multicolumn{7}{|c|}{ Earthquakes } \\
\hline & Deep. & $\begin{array}{l}\text { Inter- } \\
\text { mediate }\end{array}$ & Shallow. & $\begin{array}{l}\text { Hale- } \\
\text { maumau } \\
\text { slides }\end{array}$ & $\begin{array}{l}\text { Kilauea } \\
\text { caldera }\end{array}$ & $\begin{array}{l}\text { SW rift } \\
\text { and } \\
\text { Kaoiki }\end{array}$ & $\begin{array}{c}\text { Eastern } \\
\text { East } \\
\text { rift }\end{array}$ & $\begin{array}{l}\text { Hale- } \\
\text { maumau } \\
30 \mathrm{~km}\end{array}$ & $\begin{array}{l}\text { Kala- } \\
\text { pana } \\
\text { Trail }\end{array}$ & others \\
\hline 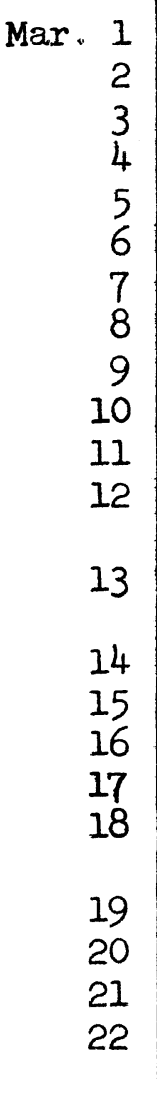 & 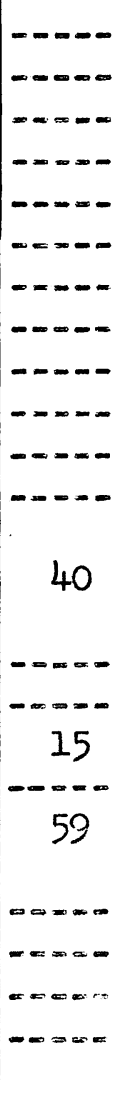 & 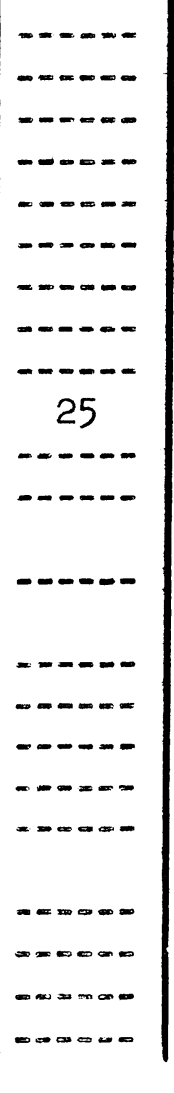 & 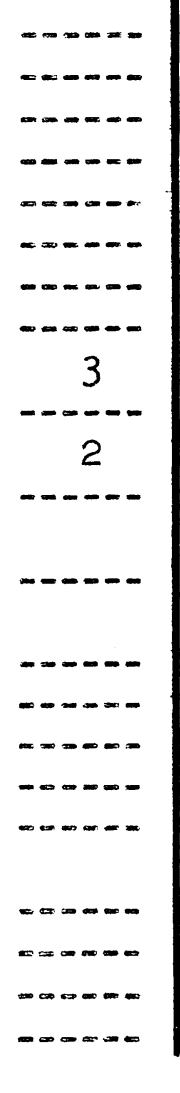 & 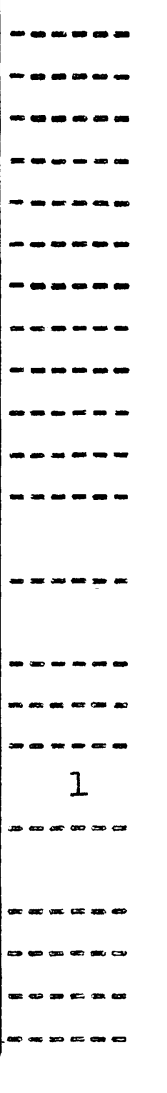 & $\begin{array}{r}50 \\
70 \\
65 \\
166 \\
100 \\
80 \\
120 \\
100 \\
70 \\
60 \\
80 \\
80 \\
\\
90 \\
\\
75 \\
100 \\
90 \\
75 \\
79 \\
76 \\
96 \\
90 \\
90\end{array}$ & $\begin{array}{r}12 \\
17 \\
11 \\
12 \\
13 \\
14 \\
20 \\
7 \\
16 \\
15 \\
30 \\
22 \\
\\
27 \\
\\
6 \\
17 \\
20 \\
20 \\
32 \\
18 \\
15 \\
15 \\
10\end{array}$ & 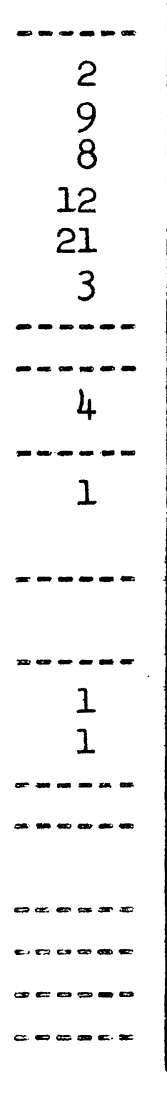 & \begin{tabular}{c}
2 \\
2 \\
$-\ldots$ \\
3 \\
3 \\
1 \\
2 \\
\hdashline 2 \\
2 \\
6 \\
2 \\
\hdashline \\
3 \\
\\
9 \\
3 \\
7 \\
1 \\
6 \\
5 \\
12 \\
10 \\
7
\end{tabular} & 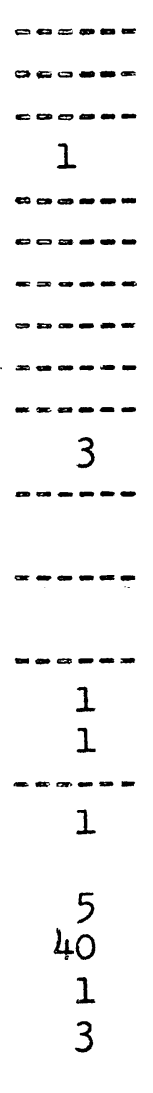 & $\begin{array}{l}4 \text { Kona } \\
1 \text { Mauna Kea } \\
1 \text { Maui } \\
1 \text { Maui } \\
1 \text { Kona } \\
1 \text { Mauna Kea } \\
1 \text { Kona } \\
1 \text { Mauna Kea } \\
3 \text { Kona } \\
1 \text { Mauna Kea } \\
-2 \text { - } \\
1 \text { Kona } \\
2 \text { Kona } \\
3 \text { Kona } \\
2 \text { Mauna Kea } \\
2 \text { Kona } \\
1 \text { Kona } \\
2 \text { Kona } \\
1 \text { Mauna Loa }\end{array}$ \\
\hline
\end{tabular}


Table 3.- Numbers of earthquakes and minutes of tremor recorded on seismographs $\mathrm{U}_{2} \mathrm{M}, \mathrm{A}, \mathrm{D}$, and $\mathrm{N}$ around Kilauea caldera--Continued

\begin{tabular}{|c|c|c|c|c|c|c|c|c|c|c|}
\hline \multirow{2}{*}{$\begin{array}{l}\text { Date } \\
(1963)\end{array}$} & \multicolumn{3}{|c|}{$\begin{array}{c}\text { Tremor } \\
\text { (in minutes) }\end{array}$} & \multicolumn{7}{|c|}{ Earthquakes } \\
\hline & Deep & $\begin{array}{l}\text { Intero } \\
\text { mediate }\end{array}$ & Shallow & $\begin{array}{l}\text { Hale- } \\
\text { maumau } \\
\text { slides }\end{array}$ & $\begin{array}{l}\text { Kilauea } \\
\text { caldera }\end{array}$ & $\begin{array}{l}\text { SW. rift } \\
\text { and } \\
\text { Kaoiki }\end{array}$ & $\begin{array}{c}\text { Eastern } \\
\text { East } \\
\text { rift }\end{array}$ & $\begin{array}{l}\text { Hale- } \\
\text { maumau } \\
30 \mathrm{~km}\end{array}$ & $\begin{array}{l}\text { Kala- } \\
\text { pana } \\
\text { Trail }\end{array}$ & Others \\
\hline $\begin{array}{r}23 \\
24 \\
\\
25 \\
26 \\
27 \\
\\
28 \\
29 \\
30 \\
31\end{array}$ & 3 & 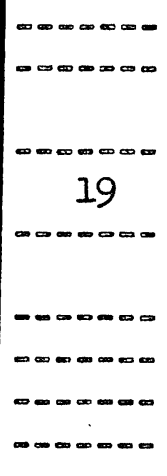 & 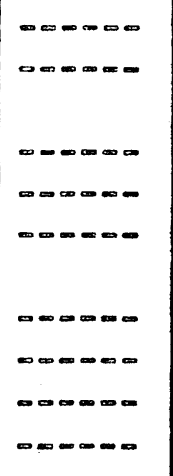 & 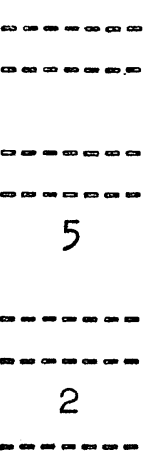 & $\begin{array}{r}80 \\
115 \\
176 \\
120 \\
153 \\
100 \\
120 \\
100 \\
3\end{array}$ & $\begin{array}{r}16 \\
11 \\
28 \\
8 \\
19 \\
\\
9 \\
8 \\
7 \\
3\end{array}$ & 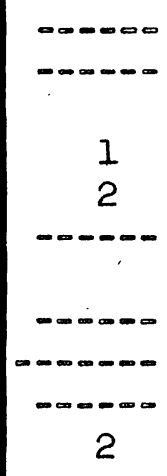 & $\begin{array}{r}10 \\
6 \\
9 \\
5 \\
5 \\
4 \\
1 \\
-9 \\
4\end{array}$ & $\begin{array}{c}-\infty \\
10 \\
16 \\
\cdots-\infty \\
9 \\
-\infty-\infty \\
\cdots-\infty \\
-\infty\end{array}$ & $\begin{array}{l}1 \text { Pohakuloa } \\
1 \text { Maui } \\
3 \text { Kona } \\
1 \text { Mauna Kea } \\
1 \text { Mauna Kea } \\
1 \text { Mauna Kea }\end{array}$ \\
\hline
\end{tabular}


Table 4.-- Local earthquakes recorded by seismographs of the U.S. Geological Survey,

$$
\text { January, February, and March, } 1963
$$

[Except for smaller earthquakes of special interest, only earthquakes with magnitudes of 2.5 or greater are listed. Origin time is Hawailan standard.

In the following list some origin times are followed only by "KM 30" and a statement of magnitude. These are all members of a continuing family of quakes noted also in other Summaries. The best mean focus for this group is beneath Halemaumau at a depth of $30 \mathrm{~km}\left(19^{\circ} 24_{.1}^{\prime} \mathrm{N} ., 155^{\circ} 17^{\prime} I^{\prime} \mathrm{W}\right.$. ).

Origin times of members of a second persistent sequence of earthquakes are followed by "Kr" (Kalapana Trail). These earthquakes originate at very shallow depths in a remote region along the Kalapana Trail west of Kalapana, and they generally are not felt. Seismograms of these earthquakes are poorly recorded and difficult to interpret; so only an approximate epicenter, $19^{\circ} 20^{\prime} \mathrm{N}_{0}, 155^{\circ} 05^{\prime} \mathrm{W}_{0}$, can be assigned to them.

The mean focus of the magnitude 6.1 Kaoiki fault system earthquake of June 27 and its aftershocks is $19^{\circ} 24^{\prime} \mathrm{N} ., 155^{\circ} 25^{\prime} \mathrm{W}$., at a depth of 3 to $8 \mathrm{~km}$. This focus has been abbreviated "Kaolki."

Since the flank eruption near Aloi Crater (Sumnary 28) numerous earthquakes have been recorded from the Aloi region and the Koae fault system southwest of Aloi. These are described as

\begin{tabular}{|c|c|c|c|c|c|c|c|c|c|}
\hline \multirow{2}{*}{$\begin{array}{l}\text { Date } \\
(1963)\end{array}$} & \multicolumn{3}{|c|}{ Time } & \multirow{2}{*}{$\begin{array}{l}\text { Magni- } \\
\text { tude }\end{array}$} & \multirow{2}{*}{ Depth } & \multicolumn{3}{|c|}{ Epicenter } & \multirow{2}{*}{$\begin{array}{c}\text { Felt } \\
\text { Report }\end{array}$} \\
\hline & $\underline{\mathrm{h}}$ & $m$ & $\underline{s}$ & & & Iat. N. & Long. W. & Description & \\
\hline $\begin{array}{r}\text { Jan. } \\
2 \\
5 \\
5 \\
8 \\
8 \\
\\
8 \\
8 \\
8 \\
8 \\
8\end{array}$ & $\begin{array}{l}03 \\
08 \\
17 \\
22 \\
07 \\
09 \\
09 \\
09 \\
09 \\
09 \\
10\end{array}$ & $\begin{array}{l}41 \\
03 \\
33 \\
27 \\
56 \\
39 \\
\\
46 \\
48 \\
49 \\
50 \\
02\end{array}$ & $\begin{array}{l}54.4 \\
00.4 \\
57.2 \\
59.4 \\
00.6 \\
44.9 \\
\\
25.3 \\
44.0 \\
25.1 \\
49.2 \\
08.5\end{array}$ & $\begin{array}{l}2.5 \\
2.9 \\
2.2 \\
2.2 \\
2.5 \\
4.3 \\
\\
2.4 \\
2.2 \\
2.3 \\
2.4 \\
2.1\end{array}$ & $\begin{array}{c}8 \\
3 \\
-\infty \\
3 \\
-\infty- \\
-\infty \\
-\infty \\
-\infty \\
-\infty \\
-\infty \\
-\infty\end{array}$ & $\begin{array}{l}19^{\circ} 11.2^{\prime} \\
19^{\circ} 13.8^{\prime} \\
19^{\circ} 48.8^{\prime} \\
-\infty \ldots \infty \\
-\infty\end{array}$ & $\begin{array}{l}155^{\circ} 37.9^{\prime} \\
155^{\circ} 35.2^{\prime} \\
155^{\circ} 20.2^{\prime} \\
\ldots-\infty\end{array}$ & 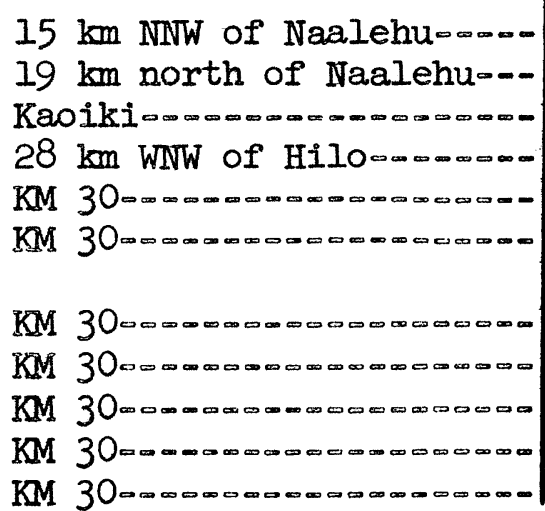 & $\begin{array}{l}\text { Felt island wide, } \\
\text { Maui and Oahu. } \\
-\infty \\
-\infty \infty \\
-\infty\end{array}$ \\
\hline
\end{tabular}


Table 4.--Iocal earthquakes recorded by seismographs of the U.S. Geological Survey, January, February, and March, 1963 --Continued

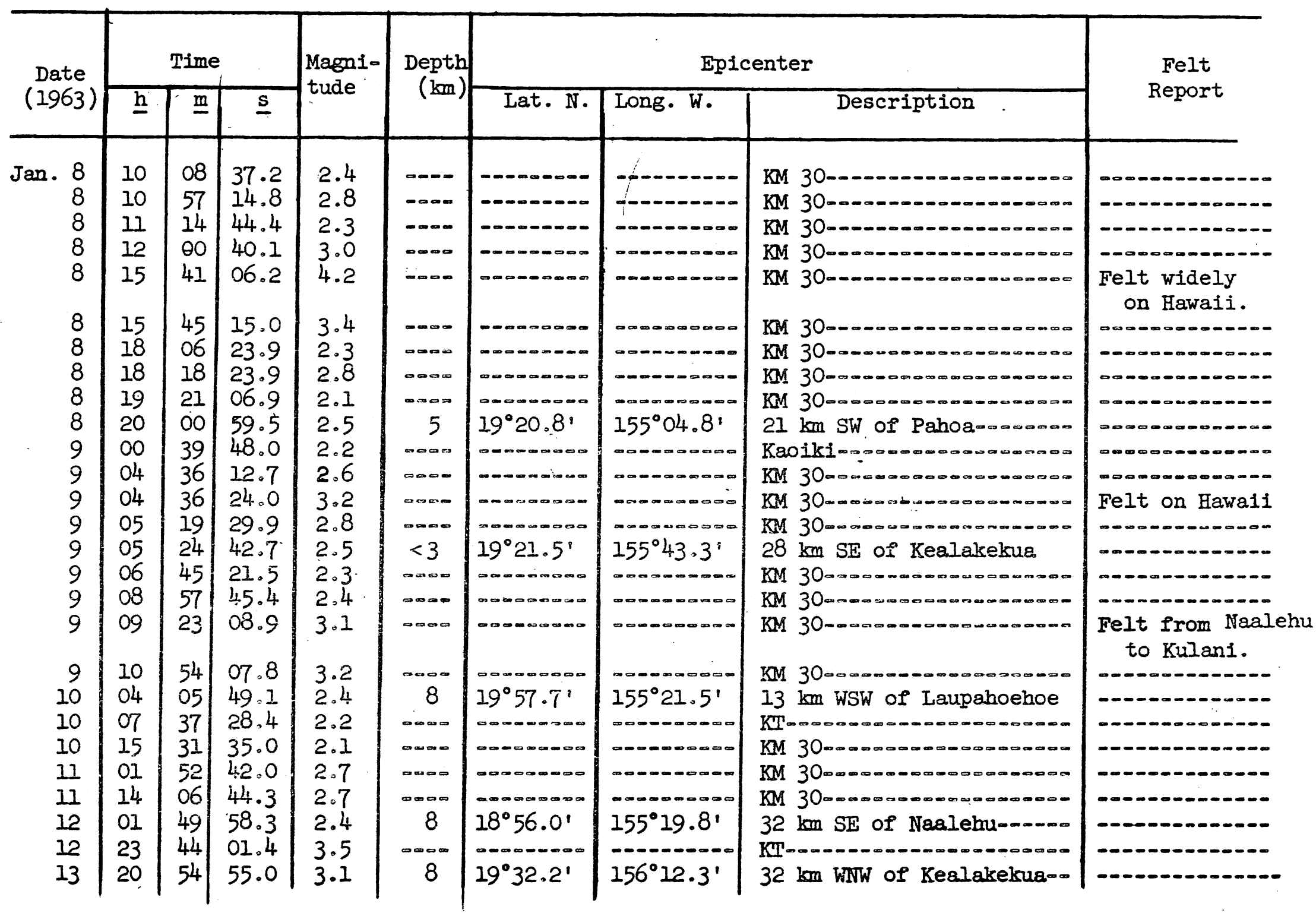


Table 4.--Local earthquakes recorded by seismographs of the U.S Geological Survey, January, February, and March, 1963--Continued

\begin{tabular}{|c|c|c|c|c|c|c|c|c|c|c|}
\hline \multirow{2}{*}{\multicolumn{2}{|c|}{$\begin{array}{l}\text { Date } \\
\text { (1963) }\end{array}$}} & \multicolumn{3}{|c|}{ Time } & \multirow{2}{*}{$\begin{array}{l}\text { Magni- } \\
\text { tude }\end{array}$} & \multirow{2}{*}{$\begin{array}{l}\text { Depth } \\
(\mathrm{km})\end{array}$} & \multicolumn{3}{|r|}{ Epicenter } & \multirow{2}{*}{$\begin{array}{l}\text { Felt } \\
\text { Report }\end{array}$} \\
\hline & & $\underline{h}$ & $\underline{m}$ & $\underline{s}$ & & & Lat. N. & Long. W. & Description & \\
\hline \multirow[t]{17}{*}{ Jan. } & & 13 & 50 & 21.0 & 2.5 & 3 & $19^{\circ} 21.9^{\prime}$ & $155^{\circ} 08.2^{\prime}$ & $14 \mathrm{~km}$ east of Ahua seismometer & $000000-000000$ \\
\hline & 15 & 03 & 21 & 20.0 & 3.2 & $-\infty-\infty$ & $-\infty-\infty-\infty$ & $-\infty-\infty-\infty$ & $\mathrm{KM} 30 \ldots \ldots \ldots$ & $-0--0-0-\infty-0-0-00-$ \\
\hline & 18 & 05 & 58 & 50.2 & 2.2 & $-\infty$ & $-\infty-\infty-\infty$ & $-\ldots-\infty-\infty$ & 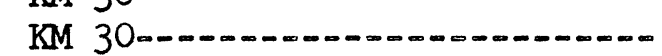 & 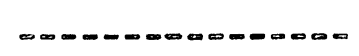 \\
\hline & 18 & 10 & 51 & 06.0 & $2 \cdot 3$ & 5 & $19^{\circ} 11.5^{\prime}$ & $155^{\circ} 37.5^{\prime}$ & $15 \mathrm{~km}$ NNW of Naalehu & $0-0-0.00000000-$ \\
\hline & 18 & 20 & 59 & 57.0 & 2.7 & 13 & $20^{\circ} 04.9^{\prime}$ & $155^{\circ} 48.9^{\prime}$ & $12 \mathrm{~km}$ WNW of Kamuela & $-\infty-\infty-\infty-\infty-\infty-\infty 0-\infty-\infty-\infty)$ \\
\hline & 20 & 10 & 26 & 35.0 & 3.1 & 13 & $20^{\circ} 13^{\prime}$ & $156^{\circ} 15^{\prime}$ & $40 \mathrm{~km}$ WSW of Upolu Point....... & $\infty+\infty 0-\infty-\infty)$ \\
\hline & 20 & 17 & 42 & 32.8 & 2.2 & $\ldots$ & $-\infty-\infty-\infty$ & $-\infty-\infty-\infty$ & $\mathrm{KM} 30 \cdots \ldots$ & $000=-0-0-0-\infty-\infty)$ \\
\hline & 23 & 06 & 12 & 41.0 & 2.8 & 8 & $19^{\circ} 27.0^{\prime}$ & $154^{\circ} 52.8^{\prime}$ & " $10 \mathrm{~km} \mathrm{SE}$ of Pahoa & Felt in Hilo and \\
\hline & 26 & 22 & 12 & 04,4 & 2.2 & 3 & $19^{\circ} 24.8^{\prime}$ & $155^{\circ} 15.7^{\prime}$ & $\mathrm{SE}$ edge of Kilauea Caldera..... & $-\infty--\infty-\infty)$ \\
\hline & 26 & 23 & 14 & 01.8 & 2.3 & 3 & $19^{\circ} 24.8^{\prime}$ & $155^{\circ} 15.7^{\prime}$ & $\mathrm{SE}$ edge of Kilauea Caldera.... & $-----\infty-\infty$ \\
\hline & 26 & 23 & 29 & 59.3 & 2.2 & $-\infty-$ & $-\infty-\infty-\infty$ & $\infty-\infty-\infty+\infty$ & 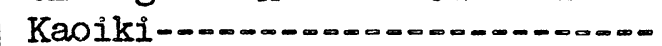 & $=---n-\infty-\infty-\infty-\infty$ \\
\hline & 27 & 13 & 58 & 05.5 & 3.4 & $\ldots-$ & $-\infty-\infty-\infty$ & $\infty-\infty-\infty-\infty$ & $\mathrm{KM} \quad 30 \cdots \cdots \cdots \cdots \infty \ldots$ & $=--\infty---0-0--\infty-\infty-0$ \\
\hline & 28 & 02 & 08 & 18.7 & 2.5 & 8 & $19^{\circ} 11.2^{\prime}$ & $155^{\circ} 34.0^{\prime}$ & $14 \mathrm{~km}$ NNE of Naalehu $\ldots \ldots$ & $--\infty--\infty--\infty \infty-\infty-\infty \infty$ \\
\hline & 29 & 12 & 53 & 19.0 & 2.3 & $-\infty$ & $-\infty-\infty \ldots$ & $-\infty-\infty$ & 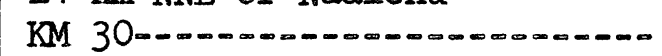 & $---\infty--\infty+\infty-\infty-\infty-\infty$ \\
\hline & 29 & 14 & 08 & 02.9 & 3.2 & $-\infty-$ & $-\infty-\infty-\infty$ & $-\cdots-\cdots-n$ & 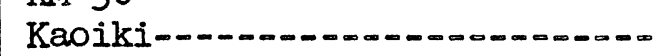 & $-m--n-0-0--m-\infty$ \\
\hline & 30 & 23 & 08 & 10.8 & 2.2 & 5 & $19^{\circ} 13 \cdot 3^{\prime}$ & $155^{\circ} 42.6^{\prime}$ & 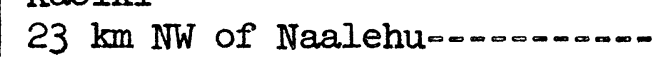 & $\infty--\infty--\infty---\infty-\infty-\infty)$ \\
\hline & 31 & 14 & 25 & 05.6 & 2.8 & 8 & $19^{\circ} 18.5^{\prime}$ & $155^{\circ} 09.5^{\prime}$ & $13 \mathrm{~km} \mathrm{SE}$ of Ahua seismometer-- & $--\infty---\infty-\infty-\infty-\infty$ \\
\hline \multirow[t]{11}{*}{ Feb。 } & 2 & 03 & 24 & $23 \cdot 3$ & 2.1 & 8 & $19^{\circ} 14^{\circ} 7^{\circ}$ & $155^{\circ} 02.0^{\prime}$ & $14 \mathrm{~km} \mathrm{SSW}$ of Kalapana $\ldots \ldots$ & $0-\infty--\infty--\infty-\infty-\infty$ \\
\hline & 4 & 12 & 48 & 44.0 & 2.1 & 13 & $20^{\circ} 19^{\prime}$ & $155^{\circ} 30^{\prime}$ & $\begin{array}{l}34 \mathrm{~km} \text { NE of Kamuela } \\
\text { seismograph. }\end{array}$ & \\
\hline & 5 & 13 & 36 & 47.8 & 2.3 & $\ldots--$ & $-\infty-\infty-\infty \cos 0$ & $-\ldots-n-n-\infty$ & Kaoiki-n & \\
\hline & 5 & 20 & 39 & 44.3 & 2.7 & 13 & $19^{\circ} 57.1^{\prime}$ & $155^{\circ} 44.8^{\prime}$ & $10 \mathrm{~km} \mathrm{SW}$ of Kamuela & \\
\hline & 5 & 22 & 47 & 25.5 & 2.2 & 5 & $19^{\circ} 13 \cdot 9^{\prime}$ & $155^{\circ} 38.2^{\prime}$ & $19 \mathrm{~km}$ NNW of Naalehu & $=-\infty-\infty$ \\
\hline & 7 & 19 & 05 & 10.9 & $2 \cdot 3$ & --- & $-\infty-\infty-\infty$ & $--\infty-\infty-\infty)$ & KT'-n-m, & $-\infty-\infty-\infty-\infty-\infty .-1$ \\
\hline & 9 & 04 & 25 & $53 \cdot 5$ & 2.5 & 8 & $19^{\circ} 35.7^{\prime}$ & $156^{\circ} 00.8^{\prime}$ & $13 \mathrm{~km} \mathrm{NW}$ of Kealakekua-..m.... & $-\cdots--n-\infty-\infty n$ \\
\hline & 9 & 10 & 19 & 53.4 & 2.0 & --- & $-\infty-\infty$ & $--\infty-2-\infty-\infty$ & 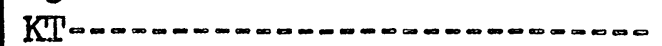 & $--m-\infty---\infty-n-\infty-\infty$ \\
\hline & 9 & 04 & 24 & 53.0 & 2.9 & 8 & $19^{\circ} 35.7^{\prime}$ & $156^{\circ} 00.8^{\prime}$ & $13 \mathrm{~km} \mathrm{NW}$ of Kealakekua $\ldots \ldots$ & $0-0-0----\infty--\infty-\infty$ \\
\hline & 10 & 18 & 49 & 48.5 & 2.7 & $-\cdots$ & $-\ldots \ldots$ & $-\ldots-\infty-\infty$ & Kaoiki-n & $-\infty-\infty-\infty-\infty-\infty m-\infty-\infty-\infty$ \\
\hline & 12 & 02 & 28 & 28.5 & 2.5 & 8 & $19^{\circ} 29.0^{\prime}$ & $155^{\circ} 58.2^{\prime}$ & $7 \mathrm{~km}$ WSW of Kealakekua............ & \\
\hline
\end{tabular}


Table 4.--Local earthquakes recorded by seismographs of the U.S. Geological Survey, January, February, and March, 1963--Continued

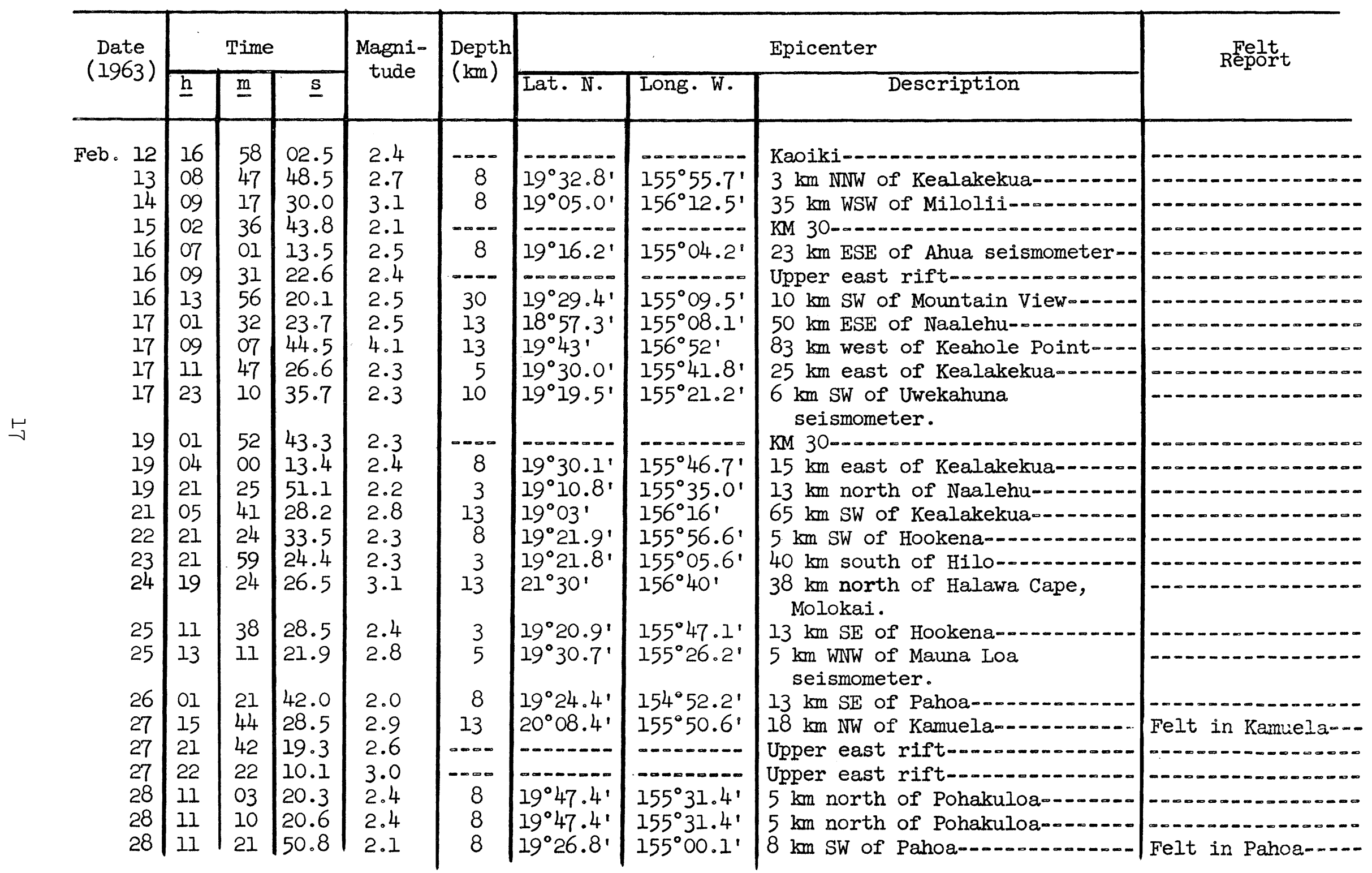


Table 4.--Iocal earthquakes recorded by seismographs of the U.S. Geological Survey, January, February, and March, 1963--Continued

\begin{tabular}{|c|c|c|c|c|c|c|c|c|c|}
\hline \multirow{2}{*}{$\begin{array}{l}\text { Date } \\
\text { (1963) }\end{array}$} & \multicolumn{3}{|c|}{ Time } & \multirow{2}{*}{$\begin{array}{l}\text { Magni- } \\
\text { tude }\end{array}$} & \multirow{2}{*}{$\begin{array}{r}\text { Depth } \\
(\mathrm{km})\end{array}$} & \multicolumn{3}{|c|}{ Epicenter } & \multirow{2}{*}{ Repolt } \\
\hline & $\underline{\underline{h}}$ & $\underline{m}$ & $\underline{s}$ & & & Lat. N. & Long. W. & Description & \\
\hline Mar. 1 & $\mathrm{OI}$ & 43 & 00.7 & $3 \cdot 2$ & 13 & $\begin{array}{l}19^{\circ} 19.8^{\prime} \\
18^{\circ} 57.3^{\prime}\end{array}$ & $\begin{array}{l}155^{\circ} 45.0^{\prime} \\
155^{\circ} 38.3^{\prime}\end{array}$ & $\begin{array}{l}17 \mathrm{~km} \mathrm{SE} \text { of Hookena } \\
5 \mathrm{~km} \mathrm{NE} \text { of Kalae Point }\end{array}$ & $\begin{array}{l}\text { Felt along south- } \\
\text { western Shore of } \\
\text { Hawaii Island. }\end{array}$ \\
\hline 3 & 06 & 14 & 38.0 & 2.3 & $\ldots$ & $--\infty-\infty-\infty$ & 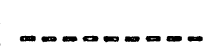 & KM 30 & n-n \\
\hline 5 & 17 & 11 & 16.4 & 2.5 & 5 & $19^{\circ} 28.5^{\circ}$ & $154^{\circ} 54.9^{\prime}$ & $5 \mathrm{~km} \mathrm{SE}$ of Pahoa & $------\infty--\infty-\infty-\infty$ \\
\hline 5 & 20 & 04 & 19.8 & 2.4 & 5 & $19^{\circ} 28.5^{\prime}$ & $154^{\circ} 54.9^{\prime}$ & $5 \mathrm{~km} \mathrm{SE}$ of Pahoa & $-----\infty-\infty-\infty-\infty---$ \\
\hline 5 & 20 & 44 & 02.3 & 2.6 & 8 & $19^{\circ} 59.0^{\prime}$ & $155^{\circ} 24.1$ & $14 \mathrm{~km}$ SE of Honokaa & 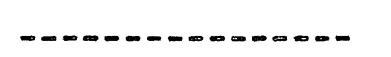 \\
\hline 6 & 02 & 23 & 55.1 & 2.8 & 5 & $19^{\circ} 28.5^{\prime}$ & $154^{\circ} 54.9^{\prime}$ & $5 \mathrm{~km}$ SE of Pahoa & Felt in Pahoa--- \\
\hline 6 & 05 & 46 & 14.3 & 2.9 & 5 & $19^{\circ} 28.5^{\prime}$ & $154^{\circ} 54.9^{\prime}$ & $5 \mathrm{~km} \mathrm{SE}$ of Pahoa & Felt in Pahoa \\
\hline 7 & 16 & 08 & 34.7 & 2.4 & 8 & $19^{\circ} 30.8^{\prime}$ & $156^{\circ} 04.8^{\prime}$ & $18 \mathrm{~km}$ west of Kealakekuam-... & $m---\infty-\infty---\infty-\infty-$ \\
\hline 8 & 14 & 25 & $23 \cdot 3$ & $2 \cdot 3$ & 8 & $19^{\circ} 17.9^{\circ}$ & $155^{\circ} 11.8^{\circ}$ & $11 \mathrm{~km} \mathrm{SE}$ of Ahua seismometer-- & $-----\infty---\infty-\infty-0-\infty$ \\
\hline 8 & 15 & 45 & 27.0 & 2.3 & 8 & $19^{\circ} 17.9^{\prime}$ & $155^{\circ} 11.8^{\circ}$ & $11 \mathrm{~km} \mathrm{SE}$ of Ahua seismometer-- & $-\infty-\infty-\infty-\infty--\infty--\infty-\infty$ \\
\hline 8 & 17 & 22 & 17.8 & 2.7 & 8 & $19^{\circ} 53.0^{\prime}$ & $155^{\circ} 31.8^{\prime}$ & $23 \mathrm{~km} \mathrm{SE}$ of Kamuela & $-\infty--\infty-\infty----\infty-\infty \infty$ \\
\hline 9 & 12 & 47 & 50.5 & 2.4 & 3 & $19^{\circ} 38.1^{\prime}$ & $156^{\circ} 04.2^{\prime}$ & $21 \mathrm{~km} \mathrm{NW}$ of Kealakekua............ & $-----\infty-\infty--\infty-\infty$ \\
\hline 10 & 11 & 51 & 52.0 & 2.3 & 8 & $19^{\circ} 14.9^{\prime}$ & $155^{\circ} 04.1^{\prime}$ & $54 \mathrm{~km}$ south of Hilo & $-----\infty--\infty-\infty-\infty--\infty$ \\
\hline 11 & 10 & 06 & 25.0 & 2.6 & 8 & $19^{\circ} 57.0^{\prime}$ & $155^{\circ} 21.9^{\prime}$ & $15 \mathrm{~km}$ WSW of Laupahoehoe.......... & Felt in Hilo-n- \\
\hline 11 & 17 & 45 & 28.3 & 3.0 & 13 & $21^{\circ} 08^{\prime}$ & $156^{\circ} 07^{\prime}$ & $\begin{array}{l}43 \mathrm{~km} \text { NNE of Haleakala } \\
\text { seismometer (Maui). }\end{array}$ & $---\infty-\infty---\infty-\infty-\infty$ \\
\hline 11 & 21 & 33 & 22.6 & 2.4 & 8 & $19^{\circ} 05.5^{\prime}$ & $155^{\circ} 21.9^{\prime}$ & $39 \mathrm{~km}$ south of Hilo & $-\infty--\infty--\infty-\infty \infty \infty-\infty-\infty)$ \\
\hline 12 & 22 & 08 & 05.2 & 2.7 & 3 & $19^{\circ} 53.1^{\circ}$ & $155^{\circ} 22.8^{\prime}$ & $6 \mathrm{~km} \mathrm{SW}$ of Keanakolu $\ldots \ldots$ & $0-\infty--\infty-\infty--\infty-\infty-\infty)$ \\
\hline 13 & 01 & 37 & $33 \cdot 5$ & 3.0 & 8 & $19^{\circ} 40.1^{\prime}$ & $155^{\circ} 50.4^{\prime}$ & $19 \mathrm{~km} \mathrm{NE}$ of Kealakekua & 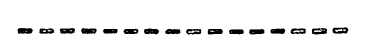 \\
\hline 13 & 03 & 22 & 52.7 & 2.3 & 3 & $19^{\circ} 51.0^{\circ}$ & $155^{\circ} 23 \cdot 3^{\prime}$ & $10 \mathrm{~km} \mathrm{SW}$ of Keanakolu $\ldots$ & $-\infty--\infty-\infty-\infty-\infty-\infty-\infty$ \\
\hline 13 & 10 & 57 & 26.9 & 3.5 & 5 & $19^{\circ} 24.2^{\prime}$ & $155^{\circ} 30.0^{\prime}$ & $\begin{array}{l}15 \mathrm{~km} \text { SW of Mauna Loa } \\
\text { seismometer. }\end{array}$ & $\begin{array}{l}\text { Felt in Pahala, } \\
\text { Hilo, and Kilauea }\end{array}$ \\
\hline 13 & 22 & 28 & 18.9 & 2.5 & 3 & $19^{\circ} 51.0^{\prime}$ & $155^{\circ} 23 \cdot 3^{\prime}$ & $10 \mathrm{~km}$ SW of Keanakolu........... & Caldéra area. \\
\hline 14 & 00 & 18 & 35.7 & $3 \cdot 3$ & 13 & $19^{\circ} 29^{\circ}$ & $157^{\circ} 14^{\prime}$ & $138 \mathrm{~km}$ west of Kealakekua-.... & 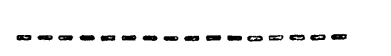 \\
\hline 14 & 01 & 23 & 26.0 & 2.1 & $\infty$ & $\infty-\infty \infty-\infty$ & $\infty-\infty \ldots+\infty$ & 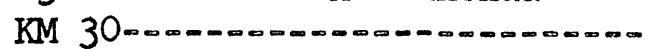 & 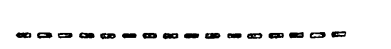 \\
\hline 14 & 08 & 23 & 20.5 & 3.0 & 8 & $20^{\circ} 12.7^{\prime}$ & $155^{\circ} 55^{\circ} 8^{\circ}$ & $31 \mathrm{~km} \mathrm{NW}$ of Kamuela $\ldots \ldots \ldots$ & $-\infty--\infty-\infty-\infty-\infty-\infty-\infty$ \\
\hline 15 & 18 & 10 & 07.0 & 2.7 & 8 & $19^{\circ} 31.0^{\prime}$ & $155^{\circ} 03.3^{\prime}$ & II $\mathrm{km} \mathrm{NW}$ of Pahoa & Felt in Pahoa-oo \\
\hline
\end{tabular}


Table 4.--Local earthquakes recorded by seismographs of the U.S. Geological Survey, January, February, and March, 1963--Continued

\begin{tabular}{|c|c|c|c|c|c|c|c|c|c|}
\hline \multirow{2}{*}{$\begin{array}{c}\text { Date } \\
(1963)\end{array}$} & \multicolumn{3}{|c|}{ Time } & \multirow{2}{*}{$\begin{array}{l}\text { Magni- } \\
\text { tude }\end{array}$} & \multirow{2}{*}{$\begin{array}{l}\text { Depth } \\
(\mathrm{km})\end{array}$} & \multicolumn{3}{|c|}{ Epicenter } & \multirow{2}{*}{$\begin{array}{c}\text { Felt } \\
\text { Report }\end{array}$} \\
\hline & $\underline{\underline{h}}$ & $\underline{\underline{m}}$ & $\underline{s}$ & & & Lat. N. & Long. W. & Description & \\
\hline $\begin{array}{r}\text { Mar. } 17 \\
17 \\
18 \\
18 \\
18 \\
19 \\
19 \\
20 \\
20 \\
20 \\
21 \\
22\end{array}$ & $\begin{array}{l}17 \\
18 \\
20 \\
21 \\
21 \\
21 \\
04 \\
10 \\
11 \\
20 \\
22 \\
\\
23 \\
06 \\
06 \\
07\end{array}$ & $\begin{array}{l}32 \\
44 \\
44 \\
50 \\
02 \\
35 \\
56 \\
17 \\
42 \\
15 \\
57 \\
59\end{array}$ & $\begin{array}{l}16.3 \\
16.9 \\
43.0 \\
56.0 \\
02.3 \\
16.5 \\
13.4 \\
34.5 \\
08.2 \\
35.9 \\
51.8 \\
06.3 \\
50.2 \\
05.6 \\
35.0\end{array}$ & $\begin{array}{l}2.5 \\
2.5 \\
2.9 \\
2.3 \\
2.4 \\
2.4 \\
2.7 \\
2.2 \\
2.6 \\
2.2 \\
2.3 \\
3.8\end{array}$ & $\begin{array}{c}<3 \\
<3 \\
--- \\
3 \\
8 \\
5 \\
8 \\
8 \\
--- \\
--- \\
<3 \\
13\end{array}$ & 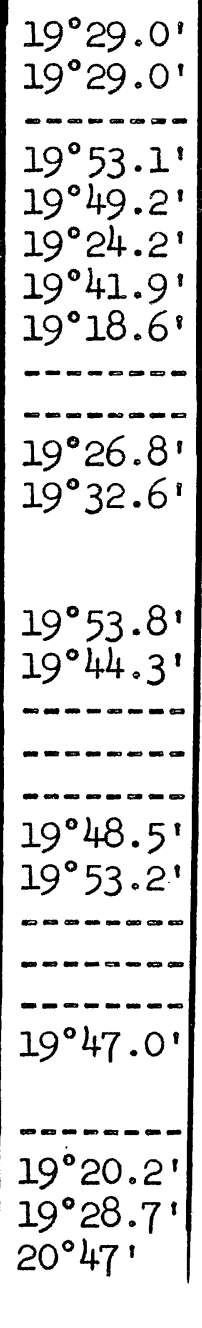 & 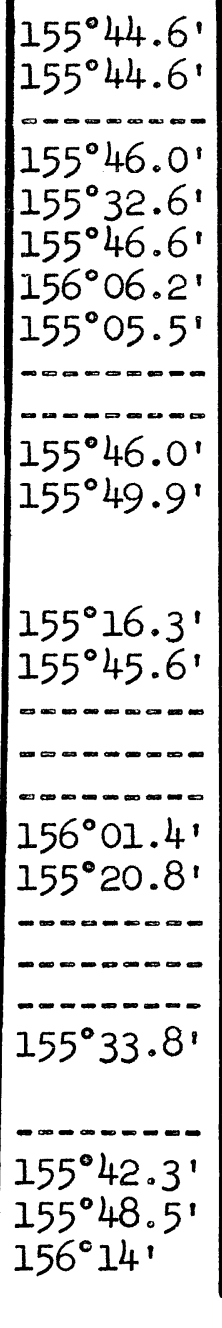 & $\begin{array}{l}28 \mathrm{~km} \mathrm{NW} \text { of Hilo } \\
30 \mathrm{~km} \text { NE of Kealakekua } \\
\text { Upper east rift } \\
\text { Upper east rift } \\
\text { Upper east rift } \\
35 \mathrm{~km} \text { NNW of Kealakekua } \\
5 \mathrm{~km} \text { SW of Keanakolu } \\
\mathrm{KM} 30 \\
\mathrm{KM} 30 \\
\mathrm{KaO} \mathrm{ki} \\
14 \mathrm{~km} \mathrm{SE} \text { of Waikii } \\
\mathrm{KM} 30 \\
22 \mathrm{~km} \text { ESE of Hookena. } \\
13 \mathrm{~km} \text { ESE of Kealakekua } \\
3 \mathrm{~km} \text { NE of Haleakala } \\
\text { seismometer (Maui). }\end{array}$ & $\begin{array}{l}\text { Kelt in Hilo, } \\
\text { Kamuela, and } \\
\text { Kealakekua. } \\
\text { Felt throughout } \\
\text { Maui. } \\
\text { and Maui. } \\
\text { Felt in Hawai1 } \\
\text { Felt in Kealakekua } \\
\text { Fa } \\
\text { Fa }\end{array}$ \\
\hline
\end{tabular}


Table 4.--Local earthquakes recorded by seismographs of the U.S. Geological Survey, January, February, and March, 1963--Continued

\begin{tabular}{|c|c|c|c|c|c|c|c|c|c|}
\hline \multirow{2}{*}{$\begin{array}{c}\text { Date } \\
\text { (1963) }\end{array}$} & \multicolumn{3}{|c|}{ Time } & \multirow{2}{*}{$\begin{array}{l}\text { Magni- } \\
\text { tude }\end{array}$} & \multirow{2}{*}{$\begin{array}{l}\text { Depth } \\
(\mathrm{km})\end{array}$} & \multicolumn{3}{|c|}{ Epicenter } & \multirow{2}{*}{$\begin{array}{c}\text { Felt } \\
\text { Report }\end{array}$} \\
\hline & $\underline{\mathrm{h}}$ & $\underline{m}$ & $\underline{\mathbf{s}}$ & & & Lat. N. & Long. W. & Description & \\
\hline $\begin{array}{r}\text { Mar. } 25 \\
26 \\
27 \\
27 \\
27 \\
28 \\
28 \\
28 \\
28 \\
29 \\
30 \\
31\end{array}$ & $\begin{array}{l}23 \\
20 \\
04 \\
11 \\
17 \\
00 \\
02 \\
13 \\
19 \\
08 \\
20 \\
16\end{array}$ & $\begin{array}{l}30 \\
56 \\
55 \\
12 \\
24 \\
26 \\
37 \\
08 \\
47 \\
50 \\
49 \\
35\end{array}$ & $\begin{array}{l}20.0 \\
19.2 \\
50.6 \\
57.0 \\
25.9 \\
22.9 \\
45.2 \\
27.1 \\
02.5 \\
08.8 \\
01.9 \\
57.5\end{array}$ & $\begin{array}{l}2.5 \\
2.5 \\
2.2 \\
2.9 \\
2.4 \\
2.3 \\
2.1 \\
2.6 \\
2.8 \\
2.1 \\
2.8 \\
3.7\end{array}$ & 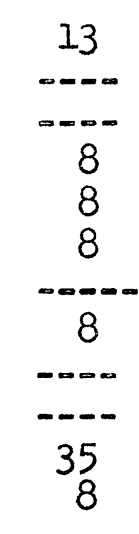 & \begin{tabular}{l}
$19^{\circ} 05.4^{\prime}$ \\
\hdashline $19^{\circ} 55.7^{\prime}$ \\
$19^{\circ} 21.4^{\prime}$ \\
$19^{\circ} 26.1^{\prime}$ \\
\hdashline $19^{\circ} 47.9^{\prime}$ \\
$19^{\circ} 09.5^{\prime}$ \\
$20^{\circ} 01.0^{\prime}$
\end{tabular} & $\begin{array}{l}155^{\circ} 25.2^{\prime} \\
155^{\circ} 32.1^{\prime} \\
155^{\circ} 45.4^{\prime} \\
155^{\circ} 36.8^{\prime} \\
155^{\circ} 34.6^{\prime} \\
155^{\circ} 30.0^{\prime} \\
155^{\circ} 52.9^{\prime}\end{array}$ & $\begin{array}{l}18 \mathrm{~km} \text { ENE of Naalehu } \\
\text { KaOiki } \\
\mathrm{KM} 30 \\
20 \mathrm{~km} \mathrm{SE} \text { of Kamuela } \\
16 \mathrm{~km} \text { ESE of Hookena } \\
5 \mathrm{~km} \mathrm{SW} \text { of Mokuaweoweo Caldera } \\
\mathrm{KM} 30 \mathrm{~km} \mathrm{NW} \text { of Pohakuloa } \\
\text { KaOiki- } \\
\text { Upper east rift } \\
14 \mathrm{~km} \mathrm{NE} \text { of Naalehu } \\
6 \mathrm{~km} \text { SW of Kawaihae }\end{array}$ & Felt in Kamuela \\
\hline
\end{tabular}


[Times are reported in Greenwich Civil Time which is 10 hours faster than Hawaiian Standard Time. A " $c$ " following the time of $P$ indicates compressional first motion; a "d" indicates dilatational first motion. Station symbols, locations, and instrumentation are presented in table 6 . Magnitudes calculated from the Hawaii seismograms are followed by (HVO). Location of epicenter, origin times, and focal depths, and magnitudes reported by other institutions are taken from "Preliminary Determination of Epicenters" published by the U.S. Coast and Geodetic Survey]

\begin{tabular}{cccl}
\cline { 1 - 2 } $\operatorname{Jan} .1$, & 1963 & & \\
\cline { 1 - 3 } & $\mathrm{Z}$ & iP & $12: 26: 51.6 \mathrm{~d}$ \\
$\mathrm{~A}$ & $\mathrm{Z}$ & $\mathrm{eP}$ & $12: 26: 50.1 \mathrm{~d}$ \\
$\mathrm{D}$ & $\mathrm{Z}$ & iP & $12: 26: 49.8 \mathrm{~d}$ \\
$\mathrm{~N}$ & $\mathrm{Z}$ & $\mathrm{eP}$ & $12: 26: 51.2 \mathrm{~d}$ \\
$\mathrm{WP}$ & $\mathrm{Z}$ & iP & $12: 26: 51.1 \mathrm{~d}$ \\
$\mathrm{Na}$ & $\mathrm{Z}$ & iP & $12: 26: 48.0$ \\
$\mathrm{~Pa}$ & $\mathrm{Z}$ & iP & $12: 26: 52.0 \mathrm{c}$ \\
$\mathrm{Hi}$ & $\mathrm{Z}$ & $\mathrm{eP}$ & $12: 26: 53.3 \mathrm{~d}$ \\
$\mathrm{Ka}$ & $\mathrm{Z}$ & $\mathrm{eP}$ & $12: 26: 54.5 \mathrm{~d}$
\end{tabular}

C\&GS card 1-63:

$12: 17: 38.6$

$6.8^{\circ} \mathrm{S} ., 155.9^{\circ} \mathrm{E}$.

Solomon Islands

$\mathrm{h}$ about $165 \mathrm{~km}$.

Jan. 1

\begin{tabular}{|c|c|c|c|c|}
\hline & & & & $14: 56: 05.4$ \\
\hline M & $\mathrm{Z}$ & eP & $23: 46: 13.6 \mathrm{~d}$ & $4.1^{\circ} \mathrm{S} ., 132.2^{\circ} \mathrm{E}$ \\
\hline A & $\mathrm{Z}$ & eP & $23: 46: 14.4 \mathrm{~d}$ & Near south coast of western New \\
\hline D & $\mathrm{Z}$ & iP & $23: 46: 14.5 \mathrm{~d}$ & Guinea. \\
\hline$N$ & $\mathrm{Z}$ & $\mathrm{eP}$ & $23: 46: 13.9 d$ & $\mathrm{~h}$ about $33 \mathrm{~km}$. \\
\hline WP & $\mathrm{Z}$ & eP & $23: 46: 13.9 \mathrm{~d}$ & \\
\hline $\mathrm{U}$ & $\mathrm{Z}$ & iP & $23: 46: 13.7 \mathrm{~d}$ & $\tan .2$ \\
\hline $\mathrm{Ha}$ & $\mathrm{Z}$ & $\begin{array}{l}\text { iP } \\
\text { epp }\end{array}$ & $23: 46: 03.4 \mathrm{c}$ & $\mathrm{U} \quad \mathrm{PEZ} \quad \mathrm{eR}$ \\
\hline $\mathrm{Ka}$ & Z & $\mathrm{eP}$ & $23: 46: 08.6$ a & C\&GS card 3-63: \\
\hline $\mathrm{Hi}$ & $\mathrm{Z}$ & iP & $23: 46: 11.4 \mathrm{~d}$ & $15: 55: 47.9$ \\
\hline $\mathrm{Pa}$ & $\mathrm{Z}$ & iP & $23: 46: 13.5 \mathrm{~d}$ & $52.9^{\circ}$ S., $118.2^{\circ}$ W. \\
\hline $\mathrm{Na}$ & $\mathrm{Z}$ & iP & $23: 46: 16.8 \mathrm{~d}$ & South Pacitic Ocean \\
\hline $\mathrm{U}$ & PEZ & iP & $23: 46: 14 \quad d$ & $33 \mathrm{~km}$. \\
\hline & & is & $23: 51: 57$ & $\operatorname{Jan} .3$ \\
\hline $\mathrm{U}$ & $\mathrm{PEF}$ & iSS & $\begin{array}{l}23: 55: 03 \\
23.52 \cdot 27\end{array}$ & $\mathrm{U} \quad \mathrm{PEZ} \quad \mathrm{eR}$ \\
\hline & & eL & $23: 54: 41$ & C\&:GS card 3-63: \\
\hline $\mathrm{U}$ & PEN & $i$ & $23: 52: 52$ & $09: 39: 46.8$ \\
\hline & & $i$ & $23: 56: 16$ & $5.3^{\circ} \mathrm{S} ., 151.5^{\circ} \mathrm{E}$. \\
\hline M & $\mathrm{Z}$ & $T \max$ & $00: 24: 45$ & New Britain \\
\hline A & $\mathrm{Z}$ & $T \max$ & $00: 24: 48$ & h about $74 \mathrm{~km}$ \\
\hline $\mathrm{D}$ & $\mathrm{Z}$ & $T \max$ & $00: 24: 40$ & Magnitude 5.3 (PaI). \\
\hline $\mathbb{N}$ & $\mathrm{Z}$ & $T \max$ & $00: 24: 48$ & \\
\hline WP & $\mathrm{Z}$ & $T \max$ & $00: 24: 44$ & \\
\hline $\mathrm{U}$ & $\mathrm{Z}$ & $T \max$ & $00: 24: 45$ & \\
\hline $\mathrm{Ha}$ & $\mathrm{Z}$ & $T \max$ & $00: 23: 07$ & \\
\hline $\mathrm{Ka}$ & Z & $T \max$ & $00: 24: 01$ & \\
\hline $\mathrm{Hi}$ & $\mathrm{Z}$ & $T \max$ & $00: 24: 11$ & \\
\hline
\end{tabular}

Jan. 1--Continued

$\mathrm{Pa} Z \mathrm{~T} \max 00: 24: 43$

C\&ESS card 1-63:

$23: 39: 05.6$

$56.6^{\circ} \mathrm{N} ., 157.7^{\circ} \mathrm{W}$.

Alaska Peninsula

$\mathrm{h}$ about $50 \mathrm{~km}$

Magnitude 6.5 (Pas)

5.75 (Pal)

6.5 (HVO)

Jan. 2

$\begin{array}{llll}M & Z & \text { iP } & 15: 07: 35.8 \mathrm{c} \\ N & Z & \text { eP } & 15: 07: 35.5 \mathrm{c}\end{array}$

C\&GS card 4-63:

$14: 56: 05.4$

$4.1^{\circ} \mathrm{S} ., 132.2^{\circ} \mathrm{E}$.

ear south coast of western New

Guinea.

h about $33 \mathrm{~km}$.

Jan. 2

GS card 3-63:

$15: 55: 47.9$

$52.9^{\circ}$ S., $118.2^{\circ} \mathrm{W}$.

South Pacific Ocean

$h$ about $33 \mathrm{~km}$.

$23: 46: 14$ d

$23: 51: 57$

$23: 55: 03$

$23: 52: 27$

23:54:41

$23: 52: 52$

$23: 56: 16$

$00: 24: 45$

$00: 24: 48$

$00: 24: 40$

$00: 24: 48$

$00: 24: 44$

$00: 24: 45$

00:23:07

$00: 24: 01$

$00: 24: 11$ 
Table 5.--Distant earthquakes--Continued

Jan. 4, 1963

$\begin{array}{llll}U & \text { PEZ } & \text { iS } & 12: 33: 51 \\ U & \text { PEZ } & \text { IR } & 12: 41: 46\end{array}$

C\&GS card 2-63: $12: 16: 38.0$

$4.7^{\circ} \mathrm{S} ., 154.0^{\circ} \mathrm{E}$. Solomon Islands region h about $69 \mathrm{~km}$.

Jan. 5

$\begin{array}{lllll}\mathrm{M} & \mathrm{Z} & \text { iP } & 00: 31: 16.7 \mathrm{c} \\ & \mathrm{Z} & \text { ipP } & 00: 32: 08.2 \mathrm{c} \\ \mathrm{A} & \mathrm{Z} & \text { eP } & 00: 31: 15.7 \mathrm{c} \\ & \mathrm{Z} & \text { epP } & 00: 32: 07.3 \mathrm{c} \\ \mathrm{H} & \mathrm{Z} & \text { eP } & 00: 31: 15.6 \mathrm{c} \\ & \mathrm{Z} & \text { epP } & 00: 32: 07.2 \mathrm{c}\end{array}$

C\&\&i card 1-63:

$00: 20: 11.6$

$3.2^{\circ} \mathrm{N} ., 127.0^{\circ} \mathrm{E}$.

Halmahera region

$\mathrm{h}$ about $33 \mathrm{~km}$.

Jan. 5

$M \quad Z$ iP $13: 29: 19.1 \mathrm{~d}$

A Z eP 13:29:18.5 d

$\mathrm{D} \quad \mathrm{Z}$ eP $13: 29: 18.1 \mathrm{~d}$

C\&GS card 2-63:

$13: 16: 38.0$

$10.0^{\circ} \mathrm{S} ., 124.0^{\circ} \mathrm{E}$.

Timor

h about $33 \mathrm{~km}$.

Jan. 7

$\begin{array}{llll}\text { M } & Z & \text { eP } & 12: 00: 27.6 \mathrm{~d} \\ \text { WP } & \text { Z } & \text { eP } & 12: 00: 27.0 \mathrm{~d} \\ \text { U } & Z & \text { eP } & 12: 00: 27.2 \mathrm{~d} \\ \mathrm{Ka} & \mathrm{Z} & \text { eP } & 12: 00: 28.8 \mathrm{~d} \\ \mathrm{I} & \text { PEZ } & \text { eR } & 12: 25: 12\end{array}$

C\&GS card 3-63:

$11: 48: 22.7$

$0.6^{\circ} \mathrm{N} ., 126.7^{\circ} \mathrm{E}$.

Halmahera region

$\mathrm{h}$ about $42 \mathrm{~km}$
Jan. 7--Continued

C\&GS card--Continued

Magnitude 5.5-5.8 ( $\mathrm{Pal}$ )

6.0 (HVO).

Jan. 9

$\mathrm{Pa} \mathrm{Z}$ eP 03:22:47.9 c

C\&GS card 4-63:

$03: 13: 26.4$

$18.6^{\circ} \mathrm{N} ., 145.4^{\circ} \mathrm{E}$.

Mariana Islands

$\mathrm{h}$ about $192 \mathrm{~km}$.

Jan. 15

$\begin{array}{lllll}\text { M } & \text { Z } & \text { eP } & 02: 42: 23.2 & \mathrm{~d} \\ \text { A } & \text { Z } & \text { eP } & 02: 42: 23.7 & \mathrm{~d} \\ \text { N } & \text { Z } & \text { eP } & 02: 42: 23.7 & \mathrm{~d} \\ \text { WP } & \text { Z } & \text { eP } & 02: 42: 23.3 & \mathrm{~d}\end{array}$

C\&GS card 4-63:

$02: 32: 39.9$

$13.4^{\circ} \mathrm{N} ., 145.3^{\circ} \mathrm{E}$.

Mariana Islands

$\mathrm{h}$ about $38 \mathrm{~km}$.

Jan. 24

$\begin{array}{lllll}\mathrm{Na} & \mathrm{Z} & \text { iP } & 12: 16: 32.5 \mathrm{c} \\ \mathrm{Hi} & \mathrm{Z} & \text { eP } & 12: 16: 40 \mathrm{~d}\end{array}$

C\&GS card 9-63:

12:09:01.2

$15.2^{\circ}$ S., $173.6^{\circ} \mathrm{W}$.

Tonga Islands region

Felt: Apia

$h$ about $33 \mathrm{~km}$.

Jan. 25

A Z eP 12:59:54.1 c

C\&GS card 7-63:

$12: 49: 42.0$

$21.8^{\circ} \mathrm{N} ., 143.8^{\circ} \mathrm{E}$.

Mariana Islands region

h about $190 \mathrm{~km}$. 
Table 5.--Distant earthquakes--Continued

\begin{tabular}{clll}
\cline { 1 - 2 } Jan. 28, 1963 & & \\
\cline { 1 - 2 } & & & \\
M & Z & eP & $12: 22: 12.8 \mathrm{~d}$ \\
$\mathrm{~A}$ & $\mathrm{Z}$ & $\mathrm{eP}$ & $12: 22: 11.9 \mathrm{~d}$ \\
$\mathrm{D}$ & $\mathrm{Z}$ & $\mathrm{eP}$ & $12: 22: 11.9 \mathrm{~d}$ \\
$\mathrm{U}$ & $\mathrm{Z}$ & $\mathrm{eP}$ & $12: 22: 12.7 \mathrm{~d}$ \\
$\mathrm{Na}$ & $\mathrm{Z}$ & $\mathrm{eP}$ & $12: 22: 11.5 \mathrm{c}$ \\
$\mathrm{Pa}$ & $\mathrm{Z}$ & $\mathrm{eP}$ & $12: 22: 13.7 \mathrm{c}$ \\
$\mathrm{Hi}$ & $\mathrm{Z}$ & iP & $12: 22: 16.2 \mathrm{c}$ \\
$\mathrm{Ha}$ & $\mathrm{Z}$ & iP & $12: 22: 16.5 \mathrm{c}$ \\
$\mathrm{U}$ & $\mathrm{PEE}$ & iS & $22: 30: 25$ \\
$\mathrm{U}$ & $\mathrm{PEZ}$ & eSS & $22: 34: 10$ \\
& & iR & $22: 38: 37$
\end{tabular}

C\&GS card 10-63:

$12: 12: 19.8$

$2.6^{\circ} \mathrm{S}:, 149.9^{\circ} \mathrm{E}$.

New Britain

h about $33 \mathrm{~km}$

Magnitude 6.5 (Pas)

6.7 (HVO).

Jan. 28

\begin{tabular}{|c|c|c|c|}
\hline M & $\mathrm{Z}$ & iP & $13: 07: 44.8 \mathrm{~d}$ \\
\hline A & $\mathrm{Z}$ & $e P$ & $13: 07: 45.7 \mathrm{~d}$ \\
\hline \multirow[t]{2}{*}{ D } & $\mathrm{Z}$ & iP & $13: 07: 45.9 \mathrm{~d}$ \\
\hline & & $i$ & $13: 08: 54.1 \mathrm{c}$ \\
\hline $\mathrm{N}$ & $\mathrm{Z}$ & $\mathrm{eP}$ & $13: 07: 45.5 \mathrm{~d}$ \\
\hline WP & $\mathrm{Z}$ & eP & $13: 07: 45.4 \mathrm{~d}$ \\
\hline \multirow[t]{3}{*}{ U } & Z & $\mathrm{eP}$ & $13: 07: 45.6 \mathrm{~d}$ \\
\hline & & ePP & $13: 09: 10.7 \mathrm{c}$ \\
\hline & & IPcP & $13: 10: 06.9 \mathrm{c}$ \\
\hline $\mathrm{Ha}$ & Z & eP & $13: 07: 40.5 \mathrm{c}$ \\
\hline $\mathrm{Hi}$ & Z & eP & $13: 07: 43.7 \mathrm{c}$ \\
\hline $\mathrm{Pa}$ & $\mathrm{Z}$ & eP & $13: 07: 44.1 \mathrm{~d}$ \\
\hline $\mathrm{Na}$ & $\mathrm{Z}$ & eP & $13: 07: 49.1 \mathrm{c}$ \\
\hline \multirow[t]{2}{*}{ U } & PEZ & is & $13: 13: 21$ \\
\hline & & iR & $13: 16: 49$ \\
\hline M & $\mathrm{Z}$ & $T \max$ & $13: 45: 33$ \\
\hline A & $\mathrm{Z}$ & $T \max$ & $13: 45: 28$ \\
\hline D & $\mathrm{Z}$ & $T \max$ & $13: 45: 30$ \\
\hline $\mathrm{N}$ & $\mathrm{Z}$ & $T \max$ & $13: 45: 11$ \\
\hline WP & $\mathrm{Z}$ & $\operatorname{Tmax}$ & $13: 45: 24$ \\
\hline $\mathrm{U}$ & $\mathrm{Z}$ & $T \max$ & $13: 45: 21$ \\
\hline $\mathrm{HA}$ & $\mathrm{Z}$ & $T \max$ & $13: 43: 33$ \\
\hline $\mathrm{Hi}$ & $\mathrm{Z}$ & $T \max$ & $13: 44: 55$ \\
\hline $\mathrm{Pa}$ & $\mathrm{Z}$ & $T \max$ & $13: 45: 08$ \\
\hline $\mathrm{Na}$ & $\mathrm{Z}$ & $T \max$ & $13: 45: 15$ \\
\hline
\end{tabular}

Jan. 28--Continued

C\&GS card 7-63:

13:00:50.7

$54.7^{\circ}$ N., $161.6^{\circ} \mathrm{E}$.

Alaska Peninsula

$\mathrm{h}$ about $33 \mathrm{~km}$

Magnitude 6.0-6.5 (Pas)

6.7 (HVO).

Jan. 29

\begin{tabular}{|c|c|c|c|}
\hline M & $\mathrm{Z}$ & iP & $09: 29: 54.6 \mathrm{~d}$ \\
\hline A & Z & $\mathrm{eP}$ & $09: 29: 55.4$ \\
\hline D & Z & $\mathrm{eP}$ & $09: 29: 54.8$ \\
\hline $\mathbb{N}$ & $\mathrm{Z}$ & eP & $09: 29: 55.0$ \\
\hline $\mathrm{U}$ & Z & eP & $09: 29: 55.1$ \\
\hline $\mathrm{Ka}$ & $\mathrm{Z}$ & iP & $09: 29: 49.4$ \\
\hline $\mathrm{Hi}$ & $\mathrm{Z}$ & iP & $09: 29: 54.0$ \\
\hline $\mathrm{Pa}$ & $\mathrm{Z}$ & iP & $09: 29: 55.6$ \\
\hline $\mathrm{Na}$ & $\mathrm{Z}$ & IP & $09: 29: 5$ \\
\hline
\end{tabular}

C\&GS card 8-63:

$09: 21: 14.3$

$49.7^{\circ} \mathrm{N} ., 154.9^{\circ} \mathrm{E}$.

Kurile Islands

h about $126 \mathrm{~km}$.

Jan. 30

$\begin{array}{llll}M & Z & \text { eP } & 10: 29: 10.9 \mathrm{c} \\ \mathrm{U} & \text { PEN } & \text { eSKSP } 10: 41: 26 \\ & & \text { iSS } & 10: 48: 36 \\ & & \text { iG } & 11: 02: 36\end{array}$

C\&GS card 10-63:

10:10:04.1

$55.6^{\circ} \mathrm{S} ., 28.3^{\circ} \mathrm{W}$.

Sandwich Islands region

h about $33 \mathrm{~km}$

Magnitude 6.5 (Pas)

7.5 (HVO).

$\underline{J a n} \cdot 31$

$\mathrm{Hi} \quad \mathrm{Z} \quad \mathrm{eP} \quad 05: 18: 03.6 \mathrm{~d}$

C\&GS card 9-63:

05:06:46.0

$27.9^{\circ} \mathrm{N} ., 126.3^{\circ} \mathrm{E}$. 
Table 5.--Distant earthquakes--Continued

Jan. 31, 1963--Continued
C\&GS card--Continued
Ryukyu Islands
h about $33 \mathrm{~km}$.

Feb. 4

$\begin{array}{llll}\text { M } & \text { Z } & \text { iP } & 23: 29: 50.5 \mathrm{~d} \\ \mathrm{~A} & \mathrm{Z} & \text { eP } & 23: 29: 51.5 \mathrm{~d} \\ \mathrm{D} & \mathrm{Z} & \text { eP } & 23: 29: 51.0 \mathrm{~d} \\ \mathrm{~N} & \mathrm{Z} & \text { eP } & 23: 29: 51.3 \mathrm{c} \\ \text { WP } & \mathrm{Z} & \text { eP } & 23: 29: 51.4 \mathrm{~d} \\ \mathrm{Hi} & \mathrm{Z} & \text { iP } & 23: 29: 49.5 \mathrm{~d} \\ \mathrm{U} & \mathrm{Z} & \text { eP } & 23: 29: 50.8 \mathrm{~d} \\ \mathrm{~Pa} & \mathrm{Z} & \text { iP } & 23: 29: 52.0 \mathrm{~d}\end{array}$

C\&GS card 10-63:

$$
\begin{aligned}
& 23: 21: 09.0 \\
& 48.5^{\circ} \mathrm{N} ., 154.9^{\circ} \mathrm{E} . \\
& \text { Kurile Islands } \\
& \mathrm{h} \text { about } 85 \mathrm{~km} .
\end{aligned}
$$

Feb. 5

$$
\begin{gathered}
\text { U PEZ eSS } 21: 10: 35 \\
\text { eR } 21: 22: 35
\end{gathered}
$$

Feb. 12

$\begin{array}{llll}\text { M } & \text { Z } & \text { iP } & 23: 14: 45.2 \mathrm{~d} \\ \mathrm{~A} & \mathrm{Z} & \text { iP } & 23: 14: 44.3 \mathrm{~d} \\ \mathrm{D} & \mathrm{Z} & \text { iP } & 23: 14: 43.6 \mathrm{~d} \\ \mathrm{~N} & \mathrm{Z} & \text { iP } & 23: 14: 44.5 \mathrm{~d} \\ \mathrm{WP} & \mathrm{Z} & \text { iP } & 23: 14: 44.3 \mathrm{~d} \\ \mathrm{U} & \mathrm{Z} & \text { iP } & 23: 14: 44.4 \mathrm{~d} \\ \mathrm{Na} & \mathrm{Z} & \text { iP } & 23: 14: 40.5 \mathrm{~d} \\ \mathrm{~Pa} & \mathrm{Z} & \text { eP } & 23: 14: 45.4 \mathrm{~d} \\ \mathrm{Hi} & \mathrm{Z} & \text { iP } & 23: 14: 50.0 \mathrm{~d} \\ \mathrm{Ha} & \mathrm{Z} & \text { iP } & 23: 14: 54.3 \mathrm{~d}\end{array}$

Feb. 12--Continued

$$
\begin{aligned}
& \text { C\&GS card 13-63: } \\
& 23: 07: 28.9 \\
& 17.8^{\circ} \mathrm{S} ., 178.6^{\circ} \mathrm{W} . \\
& \text { Fiji Islands } \\
& \text { h about } 583 \mathrm{~km} \\
& \text { Magnitude } 5.5 \text { (CGS) }
\end{aligned}
$$

Feb. 13

$\begin{array}{llll}M & Z & \text { eP } & 09: 01: 47.8 \mathrm{c} \\ \mathrm{A} & \mathrm{Z} & \text { iP } & 09: 01: 48.4 \mathrm{c} \\ \mathrm{D} & \mathrm{Z} & \text { iP } & 09: 01: 47.5 \mathrm{c} \\ \mathrm{N} & \mathrm{Z} & \text { iP } & 09: 01: 48.1 \mathrm{c} \\ \mathrm{WP} & \mathrm{Z} & \text { iP } & 09: 01: 48.0 \mathrm{c} \\ \mathrm{U} & \mathrm{Z} & \mathrm{eP} & 09: 01: 48.3 \mathrm{c} \\ \mathrm{Ha} & \mathrm{Z} & \text { iP } & 09: 01: 46.3 \mathrm{c} \\ \mathrm{Ka} & \mathrm{Z} & \mathrm{eP} & 09: 01: 44.2 \mathrm{c} \\ \mathrm{Hi} & \mathrm{Z} & \text { iP } & 09: 01: 48.7 \mathrm{c} \\ \mathrm{Pa} & \mathrm{Z} & \text { eP } & 09: 01: 49.4 \mathrm{c} \\ \mathrm{Na} & \mathrm{Z} & \text { iP } & 09: 01: 47.0 \mathrm{c} \\ \mathrm{U} & \mathrm{PEZ} & \text { iP } & 09: 01: 48.0 \mathrm{c} \\ & & \text { eR } & 09: 25: 34 \\ \mathrm{U} & \text { PEE } & \text { iS } & 09: 11: 32 \\ & & \text { iSS } & 09: 16: 41 \\ \mathrm{U} & \text { PEN } & \text { eSSS } & 09: 19: 46\end{array}$

C\&GS card 13-63:

$08: 50: 02.2$

$24.5^{\circ} \mathrm{N} ., 121.8^{\circ} \mathrm{E}$.

Northern Formosa

3 deaths and widespread damage

$\mathrm{h}$ about $33 \mathrm{~km}$

Magnitude 7.3 (Pas)

$$
\begin{aligned}
& 7.3 \text { (Brk) } \\
& 7.0-7.3 \text { ( } \mathrm{PaI}) \\
& 7.5 \text { (HVO) }
\end{aligned}
$$

Feb. 13

$\begin{array}{llll}M & Z & \text { iP } & 18: 23: 05.5 \mathrm{c} \\ \mathrm{A} & \mathrm{Z} & \text { iP } & 18: 23: 04.8 \mathrm{~d} \\ \mathrm{D} & \mathrm{Z} & \text { iP } & 18: 23: 03.9 \mathrm{~d} \\ \mathrm{~N} & \mathrm{Z} & \text { iP } & 18: 23: 05.0 \mathrm{~d} \\ \mathrm{WP} & \mathrm{Z} & \text { iP } & 18: 23: 04.9 \mathrm{~d} \\ \mathrm{Na} & \mathrm{Z} & \text { iP } & 18: 23: 02.8 \mathrm{~d} \\ \mathrm{~Pa} & \mathrm{Z} & \text { iP } & 18: 23: 06.3 \mathrm{~d}\end{array}$


Table 5.--Distant earthquakes--Continued

Feb. 13, 1963--Continued

$\begin{array}{lllll}\mathrm{Hi} & \mathrm{Z} & \text { eP } & 18: 23: 07.5 \mathrm{~d} \\ \mathrm{Ka} & \mathrm{Z} & \text { eP } & 18: 23: 06.8 \mathrm{c} \\ \mathrm{Ha} & \mathrm{Z} & \text { iP } & 18: 23: 05.8 \mathrm{c} \\ \mathrm{U} & \mathrm{PEZ} & \text { iP } & 18: 23: 06 & \mathrm{c} \\ & & \text { eR } & 18: 37: 34 & \\ \mathrm{U} & \text { PEE } & \text { iS } & 18: 30: 42 \\ \mathrm{U} & \text { PEN } & \text { eG } & 18: 35: 00\end{array}$

C\&GS card 16-63:

$18: 13: 55.1$

$9.9^{\circ} \mathrm{S.}, 160.8^{\circ} \mathrm{E}$.

Solomon Islands

$\mathrm{h}$ about $29 \mathrm{~km}$

Magnitude 6.0-6.3 (Pal)

6.5 (Pas)

6.5 (Brk)

5.8 (CGS)

6.6 (HVO).

Feb. 14

$\begin{array}{llll}\mathrm{M} & \mathrm{Z} & \text { iP } & 07: 16: 32.8 \mathrm{c} \\ \mathrm{D} & \mathrm{Z} & \mathrm{eP} & 07: 16: 31.3 \mathrm{c} \\ \mathrm{N} & \mathrm{Z} & \mathrm{eP} & 07: 16: 32.3 \mathrm{c} \\ \mathrm{WP} & \mathrm{Z} & \mathrm{eP} & 07: 16: 32.1 \mathrm{c} \\ \mathrm{U} & \mathrm{Z} & \text { iP } & 07: 16: 32.0 \mathrm{c} \\ \mathrm{Na} & \mathrm{Z} & \text { iP } & 07: 16: 30.4 \mathrm{c} \\ \mathrm{Pa} & \mathrm{Z} & \text { iP } & 07: 16: 33.9 \mathrm{~d} \\ \mathrm{Hi} & \mathrm{Z} & \text { iP } & 07: 16: 34.6 \mathrm{c} \\ \mathrm{Ka} & \mathrm{Z} & \text { iP } & 07: 16: 34.0 \mathrm{c} \\ \mathrm{Ha} & \mathrm{Z} & \text { iP } & 07: 16: 34.2 \mathrm{c}\end{array}$

C\&GS card 15-63:

$07: 04: 40.8$

$7.2^{\circ}$ S., $128.2^{\circ} \mathrm{E}$.

Banda Sea

h about $197 \mathrm{~km}$

Magnitude 6.5 (Pas)

5.8 (CGS)

Felt: Darwin, Australia.

Feb. 14

$\begin{array}{llll}\text { M } & \text { Z } & \text { iP } & 22: 18: 22.3 \mathrm{c} \\ \text { D } & \text { Z } & \text { eP } & 22: 18: 21.5 \mathrm{~d} \\ \mathrm{~N} & \mathrm{Z} & \text { eP } & 22: 18: 21.8 \mathrm{~d} \\ \mathrm{U} & \mathrm{Z} & \text { eP } & 22: 18: 21.8 \mathrm{~d} \\ \mathrm{Hi} & \mathrm{Z} & \text { eP } & 22: 18: 24.3 \mathrm{c} \\ \mathrm{U} & \text { PEN } & \text { eG } & 22: 34: 28\end{array}$

Feb. 14--Continued

U PEZ IR 22:37:14

C\&GS card 16-63:

$22: 07: 54.3$

$5.0^{\circ} \mathrm{S} ., 144.6^{\circ} \mathrm{E}$.

Eastern New Guinea

$\mathrm{h}$ about $80 \mathrm{~km}$

Magnitude 6.5 (Pas)

$6.0(\mathrm{Pal})$

$6.2($ HVO
6.0 (CGS)

Feb. 21

$\begin{array}{lllll}M & Z & \text { iP } & 02: 43: 22.7 \mathrm{c} \\ \mathrm{A} & \mathrm{Z} & \mathrm{eP} & 02: 43: 23.6 \mathrm{c} \\ \mathrm{D} & \mathrm{Z} & \mathrm{eP} & 02: 43: 23.0 \mathrm{c} \\ \mathrm{WP} & \mathrm{Z} & \text { iP } & 02: 43: 23.4 \mathrm{c} \\ \mathrm{U} & \mathrm{Z} & \mathrm{eP} & 02: 43: 23.3 \mathrm{c} \\ \mathrm{Ha} & \mathrm{Z} & \mathrm{eP} & 02: 43: 13.3 \mathrm{c} \\ \mathrm{Na} & \mathrm{Z} & \text { iP } & 02: 43: 22.3 \mathrm{c}\end{array}$

C\&GS card 14-63:

$02: 33: 35.9$

$33.4^{\circ} \mathrm{N} ., 139.2^{\circ} \mathrm{E}$.

South of Honshu, Japan

$\mathrm{h}$ about $168 \mathrm{~km}$

Magnitude 4.4 (CGS)

Feb. 21

Ha Tmax 12:42:14

C\&GS card 17-63:

12:01: 19.4

$40.4^{\circ} \mathrm{N} ., 125.0^{\circ} \mathrm{W}$.

Near coast of northern California $\mathrm{h}$ about $33 \mathrm{~km}$.

Feb. 21

$\begin{array}{llll}M & Z & \text { eP } & 13: 24: 14.6 \mathrm{~d} \\ \mathrm{~A} & \mathrm{Z} & \mathrm{eP} & 13: 24: 14.0 \mathrm{~d} \\ \mathrm{~N} & \mathrm{Z} & \mathrm{eP} & 13: 24: 14.5 \mathrm{~d} \\ \mathrm{WP} & \mathrm{Z} & \mathrm{IP} & 13: 24: 14.4 \mathrm{~d} \\ \mathrm{U} & \mathrm{Z} & \mathrm{eP} & 13: 24: 14.0 \mathrm{~d} \\ \mathrm{Hi} & \mathrm{Z} & \mathrm{iP} & 13: 24: 15.8 \mathrm{c} \\ \mathrm{Ka} & \mathrm{Z} & \mathrm{eP} & 13: 24: 16.6 \mathrm{c} \\ \mathrm{Ha} & \mathrm{Z} & \mathrm{eP} & 13: 24: 18.9 \mathrm{~d}\end{array}$


Table 5.--Distant earthquakes--Continued

Feb. 21, 1963--Continued

C\&GS card 15-63:

$13: 16: 05.6$

$20.6^{\circ} \mathrm{S} ., 175.1^{\circ} \mathrm{W}$.

Tonga Is lands region

$\mathrm{h}$ about $33 \mathrm{~km}$

Magnitude 5.2 (CGS).

Feb. 22

$\begin{array}{llll}\text { M } & \text { Z } & \text { eP } & 08: 06: 14.2 c \\ \text { A } & \text { Z } & \text { eP } & 08: 06: 13.5 c \\ \text { N } & \text { Z } & \text { iP } & 08: 06: 14.1 c \\ \text { WP } & \text { Z } & \text { iP } & 08: 06: 14.1 c \\ \text { U } & \text { Z } & \text { eP } & 08: 06: 13.9 c \\ \mathrm{Na} & \text { Z } & \text { iP } & 08: 06: 10.7 \mathrm{c} \\ \mathrm{Pa} & \text { Z } & \text { iP } & 08: 06: 15.8 c \\ \mathrm{Hi} & \text { Z } & \text { iP } & 08: 06: 16.8 \mathrm{c} \\ \mathrm{Ka} & \text { Z } & \text { iP } & 08: 06: 17.1 \mathrm{c} \\ \mathrm{Ha} & \text { Z } & \text { iP } & 08: 06: 19.8 \mathrm{c}\end{array}$

C\&GS card 16-63:

$07: 58: 57.0$

$17.8^{\circ} \mathrm{S} ., 178.8^{\circ} \mathrm{W}$.

Fiji Islands region

$\mathrm{h}$ about $550 \mathrm{~km}$

Magnitude 5.0 (CGS).

Feb'. 24

$\begin{array}{lllll}\text { M } & \text { Z } & \text { eP } & 13: 44: 18.2 \mathrm{~d} \\ \mathrm{~A} & \mathrm{Z} & \text { eP } & 13: 44: 17.4 \mathrm{~d} \\ \mathrm{D} & \mathrm{Z} & \text { eP } & 13: 44: 17.9 \mathrm{~d} \\ \mathrm{~N} & \mathrm{Z} & \text { eP } & 13: 44: 17.5 \mathrm{~d} \\ \mathrm{WP} & \mathrm{Z} & \text { eP } & 13: 44: 17.5 \mathrm{~d} \\ \mathrm{U} & \mathrm{Z} & \text { eP } & 13: 44: 17.4 \mathrm{~d} \\ \mathrm{Hi} & \mathrm{Z} & \text { iP } & 13: 44: 15.5 \mathrm{~d} \\ \mathrm{~Pa} & \mathrm{Z} & \text { iP } & 13: 44: 14.8 \mathrm{~d} \\ \mathrm{Ka} & \mathrm{Z} & \text { eP } & 13: 44: 20.9 \mathrm{~d}\end{array}$

C\&GS card 17-63:

$13: 34: 15.7$

$14.6^{\circ} \mathrm{N} ., 91.4^{\circ} \mathrm{W}$.

Central Guatemala

$\mathrm{h}$ about $135 \mathrm{~km}$

Magnitude 5.7 (CGS).
Feb. 26

$M \quad Z \quad$ iP $20: 24: 22.2 d$

A Z eP 20:24:20.7 d

$\mathrm{U} \quad \mathrm{Z}$ iP $20: 24: 22.0 \mathrm{~d}$

$\mathrm{Na} Z$ iP $20: 24: 20.4 \mathrm{~d}$

isP $20: 25: 20.5 \mathrm{c}$

$\mathrm{Pa} \quad \mathrm{Z}$ iP $20: 24: 24.3 \mathrm{~d}$

$\mathrm{Hi} \quad \mathrm{Z}$ IP $20: 24: 24.8 \mathrm{~d}$

ipP 20:25:03.0 d

$\mathrm{Ka} \quad \mathrm{Z}$ iP $20: 24: 25.6 \mathrm{~d}$

$\mathrm{Ha} Z$ iP 20:24:26.5 d

isP 20:25:17.9 c

U PEZ iP 20:24:22 d

ipP 20:25:05 d

isP 20:25:25 c

$U$ PEZ ipPP 20:27:31 an

ipPPP 20:28:46 dn

U PEE IPP 20:26:47

is $20: 32: 46$

iscs $20: 33: 21$

isS 20:34:00

U PEN IG 20:40:05

M Z Tmax 21:32:24

A Z Tmax 21:32:16

WP $\quad \mathrm{T} \quad T \max 21: 32: 16$

Ha Z Tmax 21:32:11

C\&GS card 16-63:

20:14:08.7

$7.5^{\circ} \mathrm{S} ., 146.2^{\circ} \mathrm{E}$.

Eastern New Guinea

$\mathrm{h}$ about $171 \mathrm{~km}$

Magnitude 7.3-7.5 (Pas)

7.0-7.3 (Brk)

6.8-7.0 (Pal)

7.1 (CGS)

7.0 (HVO).

Feb. 27

$\begin{array}{llll}\text { M } & \text { Z } & \text { eP } & 04: 40: 17 \\ \text { U } & \text { PEZ } & \text { eP } & 04: 40: 15 d \\ & & \text { iS } & 04: 48: 27 \\ & & \text { iSS } & 04: 52: 39 \\ & & \text { eR } & 04: 57: 55 \\ \text { U } & \text { PEE } & \text { iG } & 04: 55: 15\end{array}$


Table 5.--Distant earthquakes--Continued

Feb. 27, 1963--Continued

C\&GS card 19-63:

$04: 30: 00.8$

$6.0^{\circ} \mathrm{S} ., 149.4^{\circ} \mathrm{E}$.

New Britain region

$\mathrm{h}$ about $52 \mathrm{~km}$

Magnitude 6.5-6.8 ( $\mathrm{Pal}$ )

$$
\begin{aligned}
& 5.2 \text { (CGS) } \\
& 6.8 \text { (HVO). }
\end{aligned}
$$

Feb. 27

Ha $T$ max $28: 00: 18: 56$

C\&GS card 16-63:

$23: 36: 20.4$

$54.8^{\circ} \mathrm{N} ., 161.6^{\circ} \mathrm{W}$.

Alaska Peninsula

$\mathrm{h}$ about $33 \mathrm{~km}$

Magnitude 5.3 (CGS).

Mar. 4

$\begin{array}{llll}M & Z & \text { eP } & 15: 54: 52.8 \mathrm{~d} \\ \text { D } & Z & \text { eP } & 15: 54: 52.3 \mathrm{c} \\ \text { WP } & Z & \text { eP } & 15: 54: 52.0 \mathrm{~d} \\ . U & Z & \text { iP } & 15: 54: 52.2 \mathrm{~d}\end{array}$

C\&GS card 19-63:

$15: 43: 04.0$

$4.5^{\circ} \mathrm{S} ., 81.6^{\circ} \mathrm{W}$.

Off coast of northern Peru

$\mathrm{h}$ about $33 \mathrm{~km}$

Magnitude 5.6 (CGS).

$\underline{\operatorname{Mar} .5}$

\begin{tabular}{|c|c|c|c|}
\hline M & $\mathrm{Z}$ & iP & $07: 16: 51.7$ \\
\hline A & Z & ip & $07: 16: 50.7$ \\
\hline D & $\mathrm{Z}$ & $\mathrm{eP}$ & $07: 16: 51.0$ \\
\hline $\mathrm{N}$ & $\mathrm{Z}$ & $\mathrm{eP}$ & $07: 16: 50.8$ \\
\hline WP & $\mathrm{Z}$ & iP & 07: \\
\hline $\mathrm{U}$ & $\mathrm{Z}$ & iP & $07: 16:$ \\
\hline $\mathrm{Pa}$ & $\mathrm{Z}$ & iP & $07:$ \\
\hline $\mathrm{Hi}$ & & $\mathrm{eP}$ & 07: \\
\hline $\mathrm{Ha}$ & & iP & \\
\hline
\end{tabular}

C\&GS card 19-63:

07:05:01.7

$4.5^{\circ} \mathrm{S} ., 81.5^{\circ} \mathrm{W}$.
Mar. 5--Continued

C\&GS card--Continued

Off coast of northern Peru

$h$ about $31 \mathrm{~km}$

Magnitude 5.6 (CGS).

$\underline{\operatorname{Mar} .7}$

$M \quad Z \quad$ iP $05: 32: 18.0 \mathrm{c}$

A Z eP $05: 32: 17.4 \mathrm{c}$

D Z eP $05: 32: 17.7 \mathrm{c}$

$\mathrm{N} \quad \mathrm{Z} \quad \mathrm{eP} \quad 05: 32: 17.8 \mathrm{c}$

WP $\mathrm{Z}$ eP $05: 32: 17.8 \mathrm{c}$

U Z eP 05:32:18.0 c

$\mathrm{Pa} \quad \mathrm{Z} \quad \mathrm{eP} \quad 05: 32: 14.8 \mathrm{c}$

$\mathrm{Hi} Z$ iP $05: 32: 18.1 \mathrm{c}$

$U$ PEZ iP 05:32:19 d

isS 05:44:39

eR 05:50:05

$U$ PEE IS 05:40:41

iG $05: 47: 43$

M Z T Tmax 06:38:56

A Z T Tmax 06:38:49

D Z T $\quad$ max 06:38:55

N $\quad$ Z $\quad T \max 06: 38: 57$

WP Z Tmax 06:38:56

U Z Tmax 06:38:57

$\mathrm{Pa} \quad \mathrm{Z} \quad T \max 06: 38: 49$

$\mathrm{Na} \quad \mathrm{Z} \quad \mathrm{Tmax} \quad 06: 38: 41$

C\&GS card 18-63:

05:22:01.1

$27.0^{\circ} \mathrm{S.}, 113.5^{\circ} \mathrm{W}$.

$500 \mathrm{~km}$ west of Easter Island

h about $33 \mathrm{~km}$

Magnitude 6.8 (Pas)

6.8 (Brk)

5.6 ( CGS)

6.8 (HVO).

Mar. 7

U PEZ ePS 12:42:35

eSS $12: 47: 51$

eR 13:00:55

C\&GS card 20-63:

$12: 16: 28.5$

$44.3^{\circ} \mathrm{S}, 75.3^{\circ} \mathrm{W}$. 


Mar. 7, 1963--Continued
C\&GS card--Continued
Near coast of southern Chile
h about $45 \mathrm{~km}$
Magnitude 5.6 (CGS).

Mar. 8

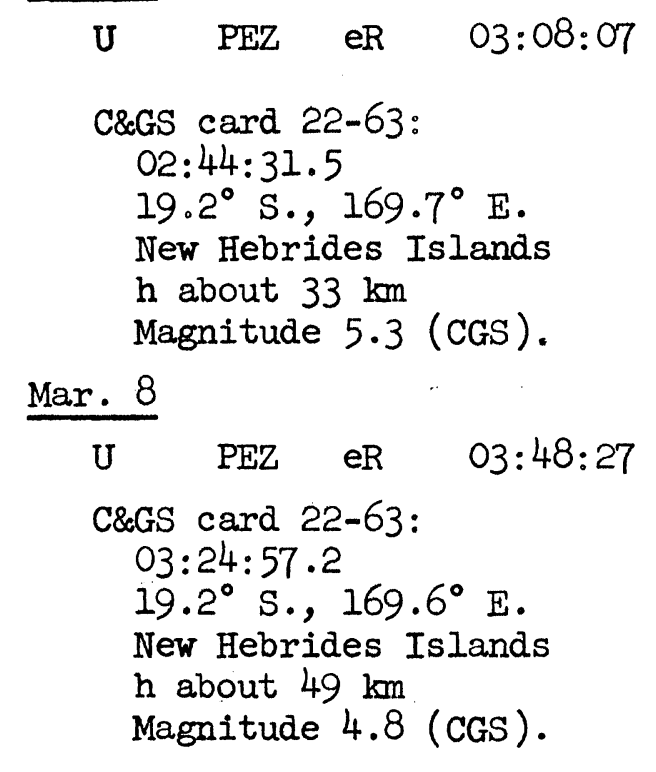

Mar. 10

$\begin{array}{llll}\text { M } & \text { Z } & T \max & 02: 12: 18 \\ \text { A } & \text { Z } & T \max & 02: 12: 26 \\ \text { D } & \text { Z } & T \max & 02: 12: 08 \\ \text { N } & \text { Z } & T \max & 02: 12: 20 \\ \text { WP } & \text { Z } & T \max & 02: 12: 18 \\ \text { U } & \text { Z } & T \max & 02: 12: 23 \\ \mathrm{~Pa} & \mathrm{Z} & T \max & 02: 11: 56 \\ \mathrm{Hi} & \mathrm{Z} & T \max & 02: 11: 40 \\ \mathrm{Ka} & \mathrm{Z} & T \max & 02: 12: 35 \\ \mathrm{Ha} & \mathrm{Z} & T \max & 02: 10: 50\end{array}$

C\&GS card 20-63:

$01: 26: 04.1$

$56.2^{\circ} \mathrm{N} ., 153.8^{\circ} \mathrm{W}$.

Kodiak Island, Alaska

$\mathrm{h}$ about $33 \mathrm{~km}$

Magnitude 5.1 (CGS).

Mar. 10

$\begin{array}{llll}\text { M } & \text { Z } & T \max & 12: 50: 12 \\ \text { A } & \text { Z } & T \max & 12: 50: 02 \\ \text { D } & \text { Z } & T \max & 12: 50: 07 \\ \text { N } & \text { Z } & T \max & 12: 50: 06 \\ \text { Na } & \text { Z } & T \max & 12: 50: 06\end{array}$

C\&GS card 20-63:
Mar. 10--Continued

C\&GS card 20-63:

10:51:48.1

$29.9^{\circ} \mathrm{S} ., 71.2^{\circ} \mathrm{W}$.

Near coast of central Chile

$\mathrm{h}$ about $70 \mathrm{~km}$

Magnitude 6.0-6.3 (Pas)

5.5 (CGS).

Mar. 10

$\begin{array}{llll}\text { M } & Z & \text { iP } & 14: 03: 01.9 \\ \text { A } & Z & \text { eP } & 14: 03: 01.7 \\ \text { N } & \text { Z } & \text { eP } & 14: 03: 01.4 \\ \text { WP } & \text { Z } & \text { eP } & 14: 03: 01.3 \\ \text { U } & \text { Z } & \text { iP } & 14: 03: 01.4\end{array}$

C\&GS card 20-63:

$13: 51: 04.3$

$2.4^{\circ} \mathrm{N} ., 126.6^{\circ} \mathrm{E}$.

Celebes sea

$\mathrm{h}$ about $41 \mathrm{~km}$.

Mar. 15

\begin{tabular}{|c|c|c|c|}
\hline M & $\mathrm{Z}$ & $\begin{array}{l}\text { iP } \\
\text { iPcP }\end{array}$ & $\begin{array}{l}00: 27: 41.0 \\
00: 27: 54.1 \mathrm{~d}\end{array}$ \\
\hline A & Z & $\begin{array}{l}\text { iP } \\
\text { iPcP }\end{array}$ & $\begin{array}{l}00: 27: 42.7 \\
00: 27: 54.4\end{array}$ \\
\hline D & Z & $\begin{array}{l}\text { iP } \\
\text { ePcP }\end{array}$ & $\begin{array}{l}00: 27: 41.7 \\
00: 27: 54.8\end{array}$ \\
\hline $\mathbb{N}$ & $\mathrm{z}$ & $\begin{array}{l}\text { eP } \\
\text { iPcP }\end{array}$ & $\begin{array}{l}00: 27: 42.2 \\
00: 27: 55.0\end{array}$ \\
\hline WP & Z & $\begin{array}{l}\text { iP } \\
\mathrm{ePcP}\end{array}$ & $\begin{array}{l}00: 27: \\
00: 27:\end{array}$ \\
\hline U & PEZ & ip & $00: 27$ \\
\hline U & PEZ & IPcP & $00: 27$ \\
\hline
\end{tabular}

C\&GS card 21-63:

$00: 16: 01.3$

$8.4^{\circ} \mathrm{N} ., 126.4^{\circ} \mathrm{E}$.

Mindanao, Philippine Islands

$\mathrm{h}$ about $117 \mathrm{~km}$

Magnitude 5.0 CGS). 


\section{Table 5.--Distant earthquakes--Continued}

\section{Mar. 16, 1963}

$\begin{array}{lllll}\mathrm{M} & \mathrm{Z} & \text { iP } & 08: 53: 36.1 \mathrm{c} \\ \mathrm{A} & \mathrm{Z} & \text { iP } & 08: 53: 37.0 \mathrm{c} \\ \mathrm{D} & \mathrm{Z} & \text { iP } & 08: 53: 36.7 & \mathrm{c} \\ \mathrm{N} & \mathrm{Z} & \text { iP } & 08: 53: 36.5 \mathrm{c} \\ \mathrm{U} & \mathrm{Z} & \mathrm{eP} & 08: 53: 36.4 \mathrm{c} \\ \mathrm{Pa} & \mathrm{Z} & \text { eP } & 08: 53: 37.0 \mathrm{~d} \\ \mathrm{Na} & \mathrm{Z} & \text { IP } & 08: 53: 38.2 \mathrm{~d} \\ \mathrm{Hi} & \mathrm{Z} & \text { iP } & 08: 53: 36.0 \mathrm{~d} \\ \mathrm{Ka} & \mathrm{Z} & \text { iP } & 08: 53: 34.1 & \mathrm{c} \\ \mathrm{Ha} & \mathrm{Z} & \text { iP } & 08: 53: 26.7 & \mathrm{c} \\ \mathrm{U} & \mathrm{PEZ} & \text { iP } & 08: 53: 37.2 & \mathrm{c} \\ \mathrm{U} & \mathrm{PEZ} & \text { I } & 08: 53: 57 & \mathrm{~d} \\ & & \text { i } & 08: 54: 13 & \mathrm{c} \\ & & \text { iPP } & 08: 55: 45 & \mathrm{~d} \\ & & \text { iS } & 09: 00: 39 & \\ & & \text { iSS } & 09: 04: 14 & \\ & & \text { eR } & 09: 07: 51 & \\ \mathrm{U} & \text { PEE } & \text { iG } & 09: 05: 13 \\ \mathrm{Ha} & \mathrm{Z} & \text { Tmax } & 09: 45: 44\end{array}$

C\&GS card 21-63:

$08: 44: 48.3$

$46.5^{\circ} \mathrm{N} ., 154.7^{\circ} \mathrm{E}$.

Kurile Islands region

$\mathrm{h}$ about $26 \mathrm{~km}$

Magnitude 7.0 (Pas)

7.8 (Brk)

$6.8(\mathrm{PaI})$

$6.2(\mathrm{CGS})$

$7 \cdot 3$ (HVO).

Mar. 20

\begin{tabular}{|c|c|c|c|}
\hline M & $\mathrm{Z}$ & eP & $04: 50: 37.6 \mathrm{~d}$ \\
\hline A & $\mathrm{Z}$ & eP & $04: 50: 36.2$ \\
\hline D & Z & eP & $04: 50: 36.5$ \\
\hline$N$ & Z & iP & $04: 50: 37.2$ \\
\hline $\mathrm{Pa}$ & $\bar{Z}$ & iP & $04: 50: 38.7$ \\
\hline $\mathrm{Hi}$ & $\mathrm{Z}$ & $\mathrm{eP}$ & $04: 50: 39.4$ \\
\hline $\mathrm{Ka}$ & $\mathrm{Z}$ & iP & $04: 50: 39.6$ \\
\hline $\mathrm{Ha}$ & $\mathrm{Z}$ & iP & $04: 50: 42.2$ \\
\hline
\end{tabular}

C\&ES card 24-63:

$04: 43: 13 \cdot 5$

$19.9^{\circ} \mathrm{S} ., 179.1^{\circ} \mathrm{W}$.

Fiji Islands region

$\mathrm{h}$ about $680 \mathrm{~km}$

Magnitude 5.2 (CGS).
Mar. 20

$\begin{array}{llll}M & Z & \text { eP } & 04: 53: 12.0 \mathrm{~d} \\ \mathrm{D} & \mathrm{Z} & \text { eP } & 04: 53: 10.9 \mathrm{~d} \\ \mathrm{~N} & \mathrm{Z} & \text { eP } & 04: 53: 11.6 \mathrm{~d} \\ \mathrm{WP} & \mathrm{Z} & \text { iP } & 04: 53: 11.6 \mathrm{~d} \\ \mathrm{U} & \mathrm{Z} & \text { eP } & 04: 53: 11.8 \mathrm{c} \\ \mathrm{Pa} & \mathrm{Z} & \text { iP } & 04: 53: 13.0 \mathrm{c} \\ \mathrm{Na} & \mathrm{Z} & \text { iP } & 04: 53: 09.5 \mathrm{c} \\ \mathrm{Hi} & \mathrm{Z} & \text { eP } & 04: 53: 14.8 \mathrm{c} \\ \mathrm{Ka} & \mathrm{Z} & \text { iP } & 04: 53: 14.9 \mathrm{c} \\ \mathrm{Ha} & \mathrm{Z} & \text { iP } & 04: 53: 17.5 \mathrm{c}\end{array}$

C\&GS card 24-63:

$04: 45: 49 \cdot 5$

$19.6^{\circ} \mathrm{S.}, 179.3^{\circ} \mathrm{W}$.

Fiji Islands region

$\mathrm{h}$ about $680 \mathrm{~km}$

Magnitude 5.2 (CGS).

Mar. 20

$M \quad Z$ iP $16: 49: 57.1 \mathrm{~d}$

C\&GS card 23-63:

$16: 38: 55.8$

$2.4^{\circ} \mathrm{S} ., 138.4^{\circ} \mathrm{E}$.

Western New Guinea

Magnitude 5.5 (CGS)

h about $40 \mathrm{~km}$.

Mar. 24

$\begin{array}{llll}\mathrm{M} & \mathrm{Z} & \text { iP } & 02: 20: 03.5 \mathrm{c} \\ \mathrm{A} & \mathrm{Z} & \text { iP } & 02: 20: 03.0 \mathrm{c} \\ \mathrm{D} & \mathrm{Z} & \text { eP } & 02: 20: 02.4 \mathrm{c} \\ \mathrm{Na} & \mathrm{Z} & \text { iP } & 02: 20: 01.9 \mathrm{c} \\ \mathrm{Ha} & \mathrm{Z} & \text { iP } & 02: 20: 08.6 \mathrm{c} \\ \mathrm{U} & \text { PEN } & \text { iL } & 02: 43: 22\end{array}$

C\&GS card 26-63:

$02: 07: 12.8$

$9.7^{\circ} \mathrm{S.}, 120.4^{\circ} \mathrm{E}$.

Sumba Island region

Magnitude 6.3 (Pas)

$6.0(\mathrm{PaI})$

5.4 (CGS)

h about $57 \mathrm{~km}$. 
Table 5.--Distant earthquakes--Continued

\begin{tabular}{cllll|}
\hline Mar. 24, 1963 & & & \\
\cline { 1 - 2 } & & & \\
M & $\mathrm{Z}$ & iP & $09: 55: 10.7$ & $\mathrm{c}$ \\
$\mathrm{A}$ & $\mathrm{Z}$ & iP & $09: 55: 11.2 \mathrm{c}$ \\
$\mathrm{D}$ & $\mathrm{Z}$ & iP & $09: 55: 10.3 \mathrm{c}$ \\
$\mathrm{Pa}$ & $\mathrm{Z}$ & iP & $09: 55: 12.8 \mathrm{c}$
\end{tabular}

C\&GS card 25-63:

$09: 43: 20.2$

$9.0^{\circ} \mathrm{N} ., 125.6^{\circ} \mathrm{E}$.

Mindanao region,

Philippine Islands

Magnitude 5.2 (CGS)

Mar. 24

$\mathrm{h}$ about $51 \mathrm{~km}$.

Mar. 26--Continued

$\begin{array}{llll}\mathrm{Pa} & \mathrm{Z} & \text { iP } & 09: 57: 40.8 \mathrm{c} \\ \mathrm{Na} & \mathrm{Z} & \text { eP } & 09: 57: 34.7 \mathrm{~d} \\ \mathrm{Hi} & \mathrm{Z} & \text { eP } & 09: 57: 41.5 \mathrm{~d} \\ \mathrm{Ka} & \mathrm{Z} & \text { eP } & 09: 57: 43.6 \mathrm{c} \\ \mathrm{Ha} & \mathrm{Z} & \text { eP } & 09: 57: 46.4 \mathrm{c} \\ \mathrm{U} & \mathrm{PEZ} & \text { iP } & 09: 57: 38 \mathrm{c} \\ & & \text { iS } & 10: 05: 15 \\ & & \text { iSS } & 10: 08: 51 \\ & \text { PEE } & \text { iG } & 10: 09: 49 \\ & & \text { iR } & 10: 12: 23\end{array}$

C\&GS card 26-63:

$09: 48: 19.7$

$29.7^{\circ} \mathrm{S} ., 177.8^{\circ} \mathrm{W}$.

Kermadec Islands

h about $45 \mathrm{~km}$

Magnitude 6.8-7.0 (Pas)

7.0 (Brk)

7.0 ( $\mathrm{Pal})$

7.1 (HVO).

Mar. 26

Ha $Z$ eP 21:42:28.8 c

U PEZ eR 21:52:03

M Z T $\max 22: 21: 23$

D Z T Tmax 22:22:05

$N \quad Z \quad T \max 22: 21: 34$

WP $Z \quad T \max 22: 21: 24$

U Z Tmax 22:21:34

$\mathrm{Pa} \quad \mathrm{Z} \quad \mathrm{Tmax} 22: 21: 32$

$\mathrm{Ha} Z$ Z Tmax 22:19:37

C\&GS card 24-63:

$21: 35: 24.4$

$51.8^{\circ} \mathrm{N} ., 178.1^{\circ} \mathrm{W}$.

Andreanof Islands,

Aleutian Islands

$\mathrm{h}$ about $57 \mathrm{~km}$

Magnitude 6.0 (Pas)

$5.0(\mathrm{PaI})$

5.5 (CGS)

5.4 (HVO).

Mar. 26

$\begin{array}{lllll}M & Z & \text { iP } & 09: 57: 39.4 & \mathrm{~d} \\ \text { A } & \text { Z } & \text { iP } & 09: 57: 38.0 & \mathrm{~d} \\ \text { D } & Z & \text { iP } & 09: 57: 37.2 & \mathrm{c} \\ \text { N } & \text { Z } & \text { eP } & 09: 57: 37.7 & \mathrm{~d} \\ \text { WP } & \mathrm{Z} & \text { eP } & 09: 57: 37.6 \mathrm{~d} \\ \text { U } & \text { Z } & \text { eP } & 09: 57: 37.3 \mathrm{~d}\end{array}$

$\begin{array}{llll}\mathrm{M} & \mathrm{Z} & \text { iP } & 13: 34: 24.2 \mathrm{~d} \\ \mathrm{~A} & \mathrm{Z} & \text { iP } & 13: 34: 22.9 \mathrm{~d} \\ \mathrm{D} & \mathrm{Z} & \mathrm{eP} & 13: 34: 22.6 \mathrm{~d} \\ \mathrm{~N} & \mathrm{Z} & \mathrm{eP} & 13: 34: 23.2 \mathrm{~d} \\ \mathrm{WP} & & \mathrm{eP} & 13: 34: 23.2 \mathrm{~d} \\ \mathrm{U} & \mathrm{Z} & \mathrm{eP} & 13: 34: 22.8 \mathrm{c} \\ \mathrm{Pa} & \mathrm{Z} & \mathrm{eP} & 13: 34: 24.9 \mathrm{~d} \\ \mathrm{Na} & \mathrm{Z} & \mathrm{eP} & 13: 34: 21.4 \mathrm{~d} \\ \mathrm{Hi} & \mathrm{Z} & \mathrm{eP} & 13: 34: 26.4 \mathrm{~d} \\ \mathrm{Ka} & \mathrm{Z} & \mathrm{eP} & 13: 34: 28.2 \mathrm{~d} \\ \mathrm{Ha} & \mathrm{Z} & \mathrm{eP} & 13: 34: 31.5 \mathrm{c} \\ \mathrm{U} & \mathrm{PEZ} & \text { iP } & 13: 34: 23 \\ & & \text { iSS } & 13: 45: 33 \\ & & \text { iR } & 13: 49: 23 \\ \mathrm{U} & \mathrm{PEN} & \text { iS } & 13: 41: 55\end{array}$

C\&GS card 27-63:

$13: 25: 02.6$

$29.8^{\circ} \mathrm{S} ., 177.9^{\circ} \mathrm{W}$.

Kermadec Islands

$h$ about $42 \mathrm{~km}$

Magnitude 7.3 (Pas)

$6.5(\mathrm{Pal})$

5.9 (CGS)

6.4 (HVO). 
Table 5.--Distant earthquakes--Continued

Mar. 26, 1963

$\begin{array}{llll}\text { M } & \text { Z } & \text { iP } & 19: 56: 56.2 \mathrm{c} \\ \mathrm{A} & \mathrm{Z} & \text { eP } & 19: 56: 57.0 \mathrm{c} \\ \mathrm{D} & \mathrm{Z} & \text { iP } & 19: 56: 56.4 \mathrm{c} \\ \mathrm{N} & \mathrm{Z} & \text { iP } & 19: 56: 56.7 \mathrm{c} \\ \mathrm{WP} & \mathrm{Z} & \text { iP } & 19: 56: 56.6 \mathrm{c} \\ \mathrm{U} & \mathrm{Z} & \text { iP } & 19: 56: 56.5 \mathrm{c} \\ \mathrm{Pa} & \mathrm{Z} & \text { eP } & 19: 56: 57.2 \mathrm{c} \\ \mathrm{Na} & \mathrm{Z} & \text { eP } & 19: 56: 57.3 \mathrm{c}\end{array}$

C\&GS card 25-63:

$19: 47: 46.0$

$44.4^{\circ} \mathrm{N} ., 146.7^{\circ} \mathrm{E}$.

Kurile Islands

$\mathrm{h}$ about $110 \mathrm{~km}$

Magnitude 5.6 (CGS)

Mar. 26

\begin{tabular}{|c|c|c|c|}
\hline M & $\mathrm{Z}$ & iP & $21: 45: 00.9$ \\
\hline A & $\mathrm{Z}$ & iP & $21: 45: 01.7$ \\
\hline D & $\mathrm{Z}$ & ip & $21: 45: 01.1$ \\
\hline $\mathrm{N}$ & $\mathrm{Z}$ & IP & $21: 45: 01.4$ \\
\hline WP & $\mathrm{Z}$ & iP & $21: 45: 01.4$ \\
\hline $\mathrm{U}$ & $\mathrm{Z}$ & iP & $21: 45: 01.2$ \\
\hline $\mathrm{Pa}$ & $\mathrm{Z}$ & iP & $21: 45: 03.1$ \\
\hline $\mathrm{Na}$ & $\mathrm{Z}$ & $1 P$ & $21: 45: 01.4$ \\
\hline $\mathrm{Hi}$ & $\mathrm{Z}$ & eP & $21: 45: 00.5$ \\
\hline $\mathrm{Ha}$ & $\mathrm{Z}$ & iP & $21: 44: 52.3$ \\
\hline \multirow[t]{2}{*}{ U } & PEZ & iP & $21: 45: 01$ \\
\hline & & iG & $22: 03: 05$ \\
\hline U & PEE & is & $21: 53: 55$ \\
\hline U & PEN & essS & $22: 00: 31$ \\
\hline
\end{tabular}

C\&GS card 24-63:

$21: 34: 41.1$

$36.0^{\circ} \mathrm{N}, 135.7^{\circ} \mathrm{E}$.

Near east coast of Honshu, Japan

Magnitude 6.0-6.3 ( $\mathrm{Pal}$ )

$$
\begin{aligned}
& 6.5 \text { (Pas) } \\
& 6.5 \text { (Brk) } \\
& 5.9 \text { (CGS) } \\
& 6.5 \text { (HVO) }
\end{aligned}
$$

Mar. 28

$\begin{array}{llll}\text { U } & \text { PEE } & \text { IS } & 00: 39: 31 \\ & & \text { II } & 00: 52: 23 \\ \text { U } & \text { PEN } & \text { IPS } & 00: 40: 36\end{array}$

Mar. 28--Continued

$\begin{array}{lll}\text { U PEZ } & \text { ISS } & 00: 45: 33 \\ & \text { iSSS } & 00: 49: 15 \\ & \text { iR } & 00: 56: 59\end{array}$

C\&GS card 27-63:

$00: 15: 47.5$

$66.3^{\circ} \mathrm{N} ., 19.6^{\circ} \mathrm{W}$.

Iceland

Magnitude 7.0-7.3 (Pas)

6.5 (Bks)

6.5-6.8 ( $\mathrm{Pal})$

$\mathrm{h}$ about $15 \mathrm{~km}$.

Mar. 28

$\begin{array}{llll}\text { M } & \text { Z } & \text { eP } & 11: 21: 52.9 \mathrm{c} \\ \text { A } & \text { Z } & \text { eP } & 11: 21: 52.5 \mathrm{c} \\ \text { WP } & \text { Z } & \text { eP } & 11: 21: 52.3 \mathrm{c} \\ \text { Hi } & \text { Z } & \text { eP } & 11: 21: 55.2 \mathrm{c}\end{array}$

C\&EGS card 27-63:

$11: 12: 31.3$

$30.2^{\circ} \mathrm{S} ., 177.8^{\circ} \mathrm{W}$.

Kermadec Islands

$\mathrm{h}$ about $38 \mathrm{~km}$.

Mar. 30

$\begin{array}{llll}\mathrm{M} & \mathrm{Z} & \text { iP } & 02: 02: 21.9 \mathrm{c} \\ \mathrm{A} & \mathrm{Z} & \mathrm{eP} & 02: 02: 21.6 \mathrm{c} \\ \mathrm{D} & \mathrm{Z} & \mathrm{eP} & 02: 02: 20.5 \mathrm{c} \\ \mathrm{N} & \mathrm{Z} & \mathrm{eP} & 02: 02: 21.6 \mathrm{c} \\ \mathrm{WP} & \mathrm{Z} & \mathrm{eP} & 02: 02: 21.6 \mathrm{c} \\ \mathrm{U} & \mathrm{Z} & \text { iP } & 02: 02: 21.4 \mathrm{c} \\ \mathrm{Na} & \mathrm{Z} & \mathrm{eP} & 02: 02: 18.5 \mathrm{c} \\ \mathrm{Hi} & \mathrm{Z} & \text { iP } & 02: 02: 24.1 \mathrm{c} \\ \mathrm{Ka} & \mathrm{Z} & \mathrm{eP} & 02: 02: 24.8 \mathrm{c} \\ \mathrm{Ha} & \mathrm{Z} & \text { iP } & 02: 02: 25.1 \mathrm{c}\end{array}$

C\&ESS card 29-63:

$01: 53: 28.8$

19.1 $1^{\circ} \mathrm{S} ., 169.1^{\circ} \mathrm{E}$.

New Hebrides Islands

Magnitude 6.1 (CGS).

$\mathrm{h}$ about $160 \mathrm{~km}$.

Mar. 30

$\mathrm{M} \quad \mathrm{Z}$ iP $17: 01: 11.4 \mathrm{~d}$ 
Table 5.--Distant earthquakes--Continued

Mar. 30, 1963--Continued

$\begin{array}{llll}\text { D } & \text { Z } & \text { iP } & 17: 01: 11.7 \mathrm{~d} \\ \mathrm{~N} & \mathrm{Z} & \text { eP } & 17: 01: 11.7 \mathrm{~d} \\ \text { WP } & \mathrm{Z} & \text { eP } & 17: 01: 11.9 \mathrm{~d} \\ \mathrm{U} & \mathrm{Z} & \text { eP } & 17: 01: 11.6 \mathrm{c} \\ \mathrm{Pa} & \mathrm{Z} & \text { iP } & 17: 01: 13.6 \mathrm{~d} \\ \mathrm{Na} & \mathrm{Z} & \text { eP } & 17: 01: 12.5 \mathrm{~d} \\ \mathrm{Hi} & \mathrm{Z} & \text { iP } & 17: 01: 11.2 \mathrm{~d}\end{array}$

C\&GS card 27-63:

$16: 51: 56.6$

$44.2^{\circ} \mathrm{N} ., 148.0^{\circ} \mathrm{E}$.

Kurile Islands

$h$ about $33 \mathrm{~km}$

Magnitude 5.3-5.5 ( $\mathrm{Pal}$ )

$$
6.3 \text { (CGS) }
$$

Mar. 30

$\begin{array}{llll}\text { M } & \text { Z } & T \max & 22: 20: 44 \\ \text { A } & \text { Z } & T \max & 22: 20: 33 \\ \text { D } & \text { Z } & T \max & 22: 20: 32 \\ \text { N } & \text { Z } & T \max & 22: 20: 30 \\ \text { U } & \text { Z } & T \max & 22: 20: 31 \\ \mathrm{~Pa} & \mathrm{Z} & T \max & 22: 20: 01 \\ \mathrm{Na} & \mathrm{Z} & T \max & 22: 20: 29\end{array}$

C\&GS card 28-63:

$21: 13: 54.1$

$8.7^{\circ}$ S., $109.2^{\circ} \mathrm{W}$.

About 2,000 km southwest of Galapagos Islands

$\mathrm{h}$ about $33 \mathrm{~km}$

Magnitude 4.6 (CGS).

Mar. 31

$\begin{array}{llll}M & Z & \text { iP } & 05: 40: 05.6 \mathrm{~d} \\ \mathrm{~A} & \mathrm{Z} & \mathrm{eP} & 05: 40: 02.2 \mathrm{~d} \\ \mathrm{~N} & \mathrm{Z} & \mathrm{eP} & 05: 40: 02.7 \mathrm{~d} \\ \mathrm{U} & \mathrm{Z} & \mathrm{eP} & 05: 40: 03.4 \mathrm{~d} \\ \mathrm{~Pa} & \mathrm{Z} & \text { eP } & 05: 40: 07.3 \mathrm{c} \\ \mathrm{U} & \text { PEN } & \text { iS } & 05: 47: 36 \\ \mathrm{U} & \text { PEZ } & \text { eR } & 05: 54: 44\end{array}$

C\&GS card 27-63:

$05: 30: 49 \cdot 3$

$29.9^{\circ} \mathrm{S} ., 177.7^{\circ} \mathrm{W}$.

Kermadec Islands

h about $48 \mathrm{~km}$.
Mar. 31--Continued

$$
\begin{aligned}
& \text { C\&GS card--Continued } \\
& \text { Magnitude } 6.3-6.5 \text { (Pas) } \\
& 6.5 \text { (Bks) } \\
& 6.0-6.3 \text { (Pal) } \\
& 5.7 \text { (CGS) } \\
& 6.3 \text { (HVO) }
\end{aligned}
$$

Mar. 31

$\begin{array}{llll}D & \text { Z } & \text { IP } & 07: 18: 13.7 \mathrm{~d} \\ \mathrm{U} & \text { PEN } & \text { IG } & 07: 32: 48 \\ \mathrm{U} & \text { PEE } & \text { eR } & 07: 35: 32\end{array}$

C\&GS card 27-63:

$07: 07: 36.3$

$6.1^{\circ}$ S., $149.0^{\circ} \mathrm{E}$, New Britain

Magnitude 6.3 (Pas)

6.0 ( $\mathrm{PaI})$

5.7 (CGS).

Mar. 31

$\begin{array}{lllll}\text { M } & \text { Z } & \text { iP } & 19: 32: 13.9 & \mathrm{~d} \\ \mathrm{~A} & \mathrm{Z} & \text { eP } & 19: 32: 12.9 \mathrm{~d} \\ \mathrm{D} & \mathrm{Z} & \text { eP } & 19: 32: 12.2 \mathrm{~d} \\ \mathrm{~N} & \mathrm{Z} & \text { iP } & 19: 32: 13.4 \mathrm{~d} \\ \text { WP } & \mathrm{Z} & \text { iP } & 19: 32: 13.4 \mathrm{~d} \\ \mathrm{U} & \mathrm{Z} & \text { iP } & 19: 32: 13.4 \mathrm{~d} \\ \mathrm{Hi} & \mathrm{Z} & \text { eP } & 19: 32: 14.9 \mathrm{~d}\end{array}$

C\&GS card 27-63:

$19: 22: 53 \cdot 3$

$30.0^{\circ} \mathrm{S} ., 178.0^{\circ} \mathrm{W}$.

Kermadec Islands

$h$ about $50 \mathrm{~km}$

Magnitude 6.3-6.5 (Pas)

$$
\begin{aligned}
& 6.5 \text { (Bks) } \\
& 5.8 \text { (CGS). }
\end{aligned}
$$


Table 6.--U.S. Geological Survey seismograph stations in Hawail

\begin{tabular}{|c|c|c|c|c|c|}
\hline \multirow{2}{*}{ Station } & \multirow{2}{*}{ Symbol } & \multicolumn{2}{|c|}{ Location } & \multirow{2}{*}{$\begin{array}{l}\text { Altitude } \\
\text { (M) } \\
\text { above sea } \\
\text { level }\end{array}$} & \multirow{2}{*}{$\begin{array}{c}\text { Equipment } \\
\text { (z, vertical; N, north-south; } \\
\text { E, east-west) }\end{array}$} \\
\hline & & $\begin{array}{c}\text { Latitude } \\
\text { N. }\end{array}$ & $\begin{array}{c}\text { Longitude } \\
\text { W. }\end{array}$ & & \\
\hline $\begin{array}{l}\text { Uwekahuna } \\
\text { (Hawaiian Volcano } \\
\text { Observatory). }\end{array}$ & $\mathrm{U}$ & $19^{\circ} 25.4^{\prime}$ & $155^{\circ} 17.6^{\prime}$ & 1,240 & $\begin{array}{l}\text { Long-period Press-Ewing: N, E, Z. } \\
\text { (Seismometer and galvanometer } \\
\text { periods are } 15 \text { and } 90 \text { seconds, } \\
\text { respectively.) } \\
\text { Short-period Sprengnether: E, Z. } \\
\text { HVO-1: ZI/. } \\
\text { Short-base liquid-level tiltmeter. } \\
\text { April 9, 1963 to May } 27,1963 \text { a } \\
\text { Wood-Anderson (NS) replaced Sp-Z } \\
\text { on an experimental, temporary } \\
\text { basis. } \\
\text { Operated by John Forbes, Akira } \\
\text { Yamamoto and other HVO staff } \\
\text { members. }\end{array}$ \\
\hline Mauna Loa-.......- & M & $19^{\circ} 29.8^{\prime}$ & $155^{\circ} 23 \cdot 3^{\prime}$ & 2,010 & Remote recording HVO-2: $\mathrm{z}^{2}$. \\
\hline Ahua & A & $19^{\circ} 22.4^{\prime}$ & $155^{\circ} 15.9^{\prime}$ & 1,070 & Remote recording HVO-2: $\mathrm{Z}$. \\
\hline Desert-n- & $\mathrm{D}$ & $19^{\circ} 20.2^{\prime}$ & $155^{\circ} 23 \cdot 3^{\prime}$ & 815 & Do. \\
\hline North Pit-........ & $\mathrm{N}$ & $19^{\circ} 24.9^{\prime}$ & $155^{\circ} 17.0^{\prime}$ & 1,115 & Do. \\
\hline West Pitw-...- & WP & $19^{\circ} 24.7^{\prime}$ & $155^{\circ} 17 \cdot 5^{\prime}$ & 1,110 & $\begin{array}{l}\text { Do. } \\
\text { Installed October 31, } 1962 .\end{array}$ \\
\hline Whitney Vault_..... & $\mathrm{W}$ & $19^{\circ} 25.9^{\prime}$ & $155^{\circ} 15.7^{\prime}$ & 1,210 & $\begin{array}{l}\text { Bosch-Omori: N, E. (Seismometer } \\
\text { period } 9 \text { seconds.) Discontinued } \\
\text { February } 1,1963 .\end{array}$ \\
\hline
\end{tabular}


Table 6.--U.S. Geological Survey seismograph stations in Hawaii--Continued

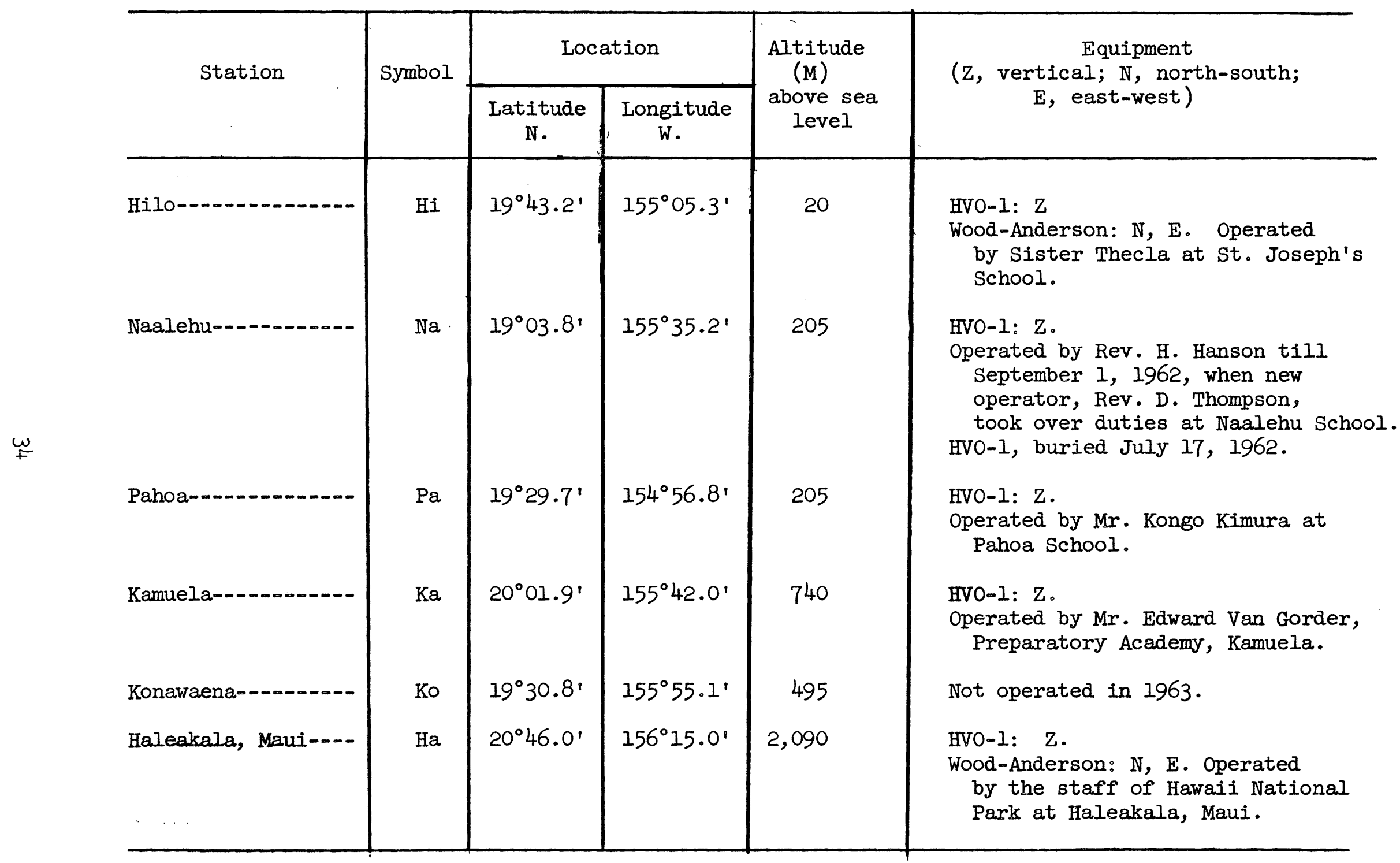

See footnotes at end of table, p. 35 . 
Table 6.--U.S. Geological Survey seismograph stations in Hawaii--Continued

I/ HVO-I is a moving-coil, hinged, vertical-component seismograph with seismometer and galvanometer periods of 0.5 second. Over-damping of both seismometer and galvanometer is used to control the strong galvanometer reaction. This seismograph has a peak magnification of about 20,000 at a period of 0.25 second. Recording is optical, on photographic paper.

2/ HVO-2 is a moving-coil, vertical-component seismograph with a seismometer period of 0.8 second. Its signal is transmitted over telephone wires to the Hawailan Volcano Observatory, where it is recorded on smoked paper. The response of this seismograph is similar to that of HVO-1. Records from these seismographs at the M, A, and D stations are recorded on a 3-component drum to permit an accurate comparison of arrival times at these stations. 
The following persons or agencies reported "felt" earthquakes during the first quarter, 1963. Their assistance is gratefully acknowledged.

North Hawaii

Honokaa School

Mrs. Hunter

Mr. Van Gorder

Mrs. Lindsey

Miss Tulley

Mrs. Thwine

Mrs. Richards, Jr.

Mrs . Christensen

Mrs. Walker

Hawaii Preparatory Academy

$\mathrm{Mr}$. McCabe

Mrs. Weight

Mrs. Eklund

Miss Wallace

Mr. Stewart

\section{Kilauea summit region}

Mrs. Loucks

Keakelani School

Mrs. Hansen

National Park Headquarters

Volcano House Hotel

Mrs. Mist

Mr. Koyanagi

Shipman Ranch (Keaau)

Mrs. Wentworth

Mrs. Duncan

Kilauea Military Camp

Miss English

Mrs. Fraser

Mrs. Yamamoto

Kona coast

Mr. Johnson, Jr.

$\mathrm{Mr}$. Sutherland

Miss Greenwell

Mr. Paris

Mrs. Mitchell

Mr. S. Greenwell

Mrs. Rice

Mr. Sleightholm

Mr. Yeoman

Mrs. Higashihara

Mrs. Cherry

Mrs. Miyatake

Mrs. Hayashi

Mrs. Yamasaki

Mrs. Korenaga
Puna

Mr. Edwards

Mr. Hay

Miss' Takemoto

Mrs. Isbell

Mr. Warmer

Mrs. Walker

Hilo region

Mr. Sadamoto

Mr. McMurray

$\mathrm{Mr}$. Pierce

Miss Perriera

Mrs. Schaeffer

Mrs. Duncan

Mr. Elliot

Mrs. Ingledue

Mr. Okamura

$\mathrm{Mr}$. Warner

Mrs. Baldwin

$\mathrm{Mr}$. Ho

$\mathrm{Mr}$. Onuma

Mrs. Veriato

Mrs. Breyton

$\underline{\text { Kau region }}$

Mr. Godfrey

Mrs. Schattauer

Mrs. Billings

Mrs. Yamamoto

Kau Police Dept.

Mr. Manierre

Mr. Edwards

\section{Central Hawaii}

Kulani Honor Camp

It. Carvalho

Mr. Kamiko

Puu Anahulu School
Mrs. Boyum

Mr. Griffiths

$\mathrm{Mr}$. Ching

Dr. Leekrick

Ulupalakua Ranch

Hana-Maui Hotel

Haleakala National Park

Mr. Hupp

Mrshizuindsay

Oahu Island

Mr. Johnson 


\section{HAWAIIAN VOLCANO OBSERVATORY \\ SUMMARY 30 \\ April, May, and June 1963 \\ By}

Harold L. Krivoy, Willie T. Kinoshita, Arnold T. Okamura and Robert Y. Koyanagi

\section{Issued March 1965}

\section{Observatory Staff}

\section{$\underline{\text { Geology }}$}

Geochemistry

J. G. Moore (Scientist-in-Charge)

R. T. Okamura

C. K. Wentworth

Geophysics

Support

- W. T. Kinoshita (Arrived from Menlo

E. T. Endo Park in June)

J. C. Forbes

G. Ko.jima

W. H. Francis

R. Y. Koyanagi

B. J. Loucks

H. L. Krivoy

A. Yamamoto

A. T, Okamura

A. S. Ryall

N. Sherrill 
Chronological summary-

Tilting of the ground around Kilauea caldera-1..- 5

Seismic summary-a 9

Persons or agencies reporting felt earthquakes during the quarter--34

Illustrations

Figure 1. Map of the island of Hawaii showing seismograph stations and localities mentioned in the text-1............. 4

2. Tilting of the ground around Kilauea caldera-...-.-.-. 8

\section{Tables}

Table A. Tilt coordinates and cumulative changes at Uwekahuna Vault, May 8-13-1

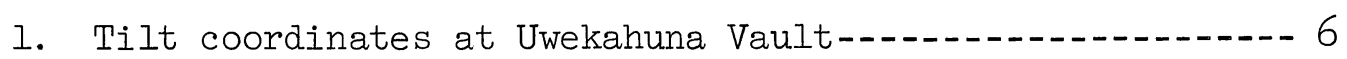

2. Tilt coordinates and changes at bases around Kilauea caldera-1-

3. Number of earthquakes and minutes of tremor recorded

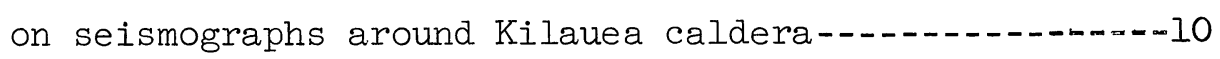

4. Local earthquakes recorded by seismographs of the U.S. Geological Survey-_.-.

5. Distant earthquakes-a2 
Slight inflation of Kilauea volcano during early April was indicated by northwestward tilting at Uwekahuna. Very heavy rainfall (26 inches in one week) was followed by moderate but persistent southward tilting between April 17-30. Part of the apparent collapse may have been caused by cooling and shrinking of near-surface lava storage, but most of it is best attributed to rainfall-induced deformation in the immediate vicinity of Uwekahuna Vault.

April was not an unusual month seismically: shallow caldera quakes were slightly more numerous but activity elsewhere was similar to that during March (table 3). Three earthquakes were felt in Hawaii during the month (table 4 ).

Strong northward tilting at Uwekahuna characterized the first 8 days of May. Except for slightly increased activity along the Kaoiki fault, including a magnitude 3.4 "felt" earthquake on March 4, the statistics of shallow and deep seismic activity continued unchanged from April.

At $21^{h} 50^{m}$ on May 9, an episode of unusual seismic activity consisting of frequent earthquakes in a background of continuous harmonic tremor began. Early in this crisis, which lasted 4 days, about a dozen earthquakes were felt lightly in the Kilauea summit region. Only two of these could be identified individually (table 4). Otherwise, earthquakes were not felt, although they occurred at rates as high as five per minute. The appearance of harmonic tremor on instruments of the Kilauea summit network suggested that lava was moving at a shallow depth beneath the summit region. Earthquakes that could be read through the tremor background originated along the Koae fault system in the vicinity of Puu Koae on Kilauea's southwest flank (fig. 2). Fresh cracks up to 2 feet in width were eventually found in this region in a zone several miles long. The cracks were all tensional. The tilt diagram for March 18 to May 12 (fig 2) very clearly shows tumescence of an elongate zone along the southwest rift zone. Movement of lava into this region from the summit reservoir is believed to have caused the seismic activity and extensive cracking.

While the southwest rift zone was swelling and cracking, a remarkable collapse of the Kilauea summit region was recorded by the short-base tiltmeter at Uwekahuna. Although the summit collapse is shown in a gross manner (7-day averages) in table 1 , a more detailed record of this event is provided by the unaveraged tilt coordinates at Uwekahuna Vault derived from a stepped-up reading schedule during the collapse (table A). On May 12 summit collapse ceased and rapid reinflation began, as indicated by rapid northwestward tilting at Uwekahuna. A remarkably similar episode of summit collapse accompanied by earthquakes and ground cracking south of the caldera occurred in December 1950 (Volcano Letter 510).

The frequency of earthquakes in and near Kilauea caldera decreased sharply after the May 9-12 crisis. Three additional earthquakes were felt on Hawaii during the rest of the month.

During June, seismic activity in the vicinity of Kilauea caldera and along the upper part of Kilauea's east rift zone increased slightly. Tilting at Uwekahuna declined about the end of May and showed little change during June. 
Four earthquakes were felt on Hawaii during June. The largest was of magnitude 4.2 and occurred beneath the south flank of Mauna Loa on June 6.

Note on instrumentation.--Early in April an HVO-2 seismometer was installed at Makaopuhi Crater. Its signal is transmitted over a telephone line to the Observatory, where recording of data from the new station (MP) was begun on April 18.

Table A.--Tilt coordinates and cumulative changes at Uwekahuna Vault during the May 9-12 summit collapse

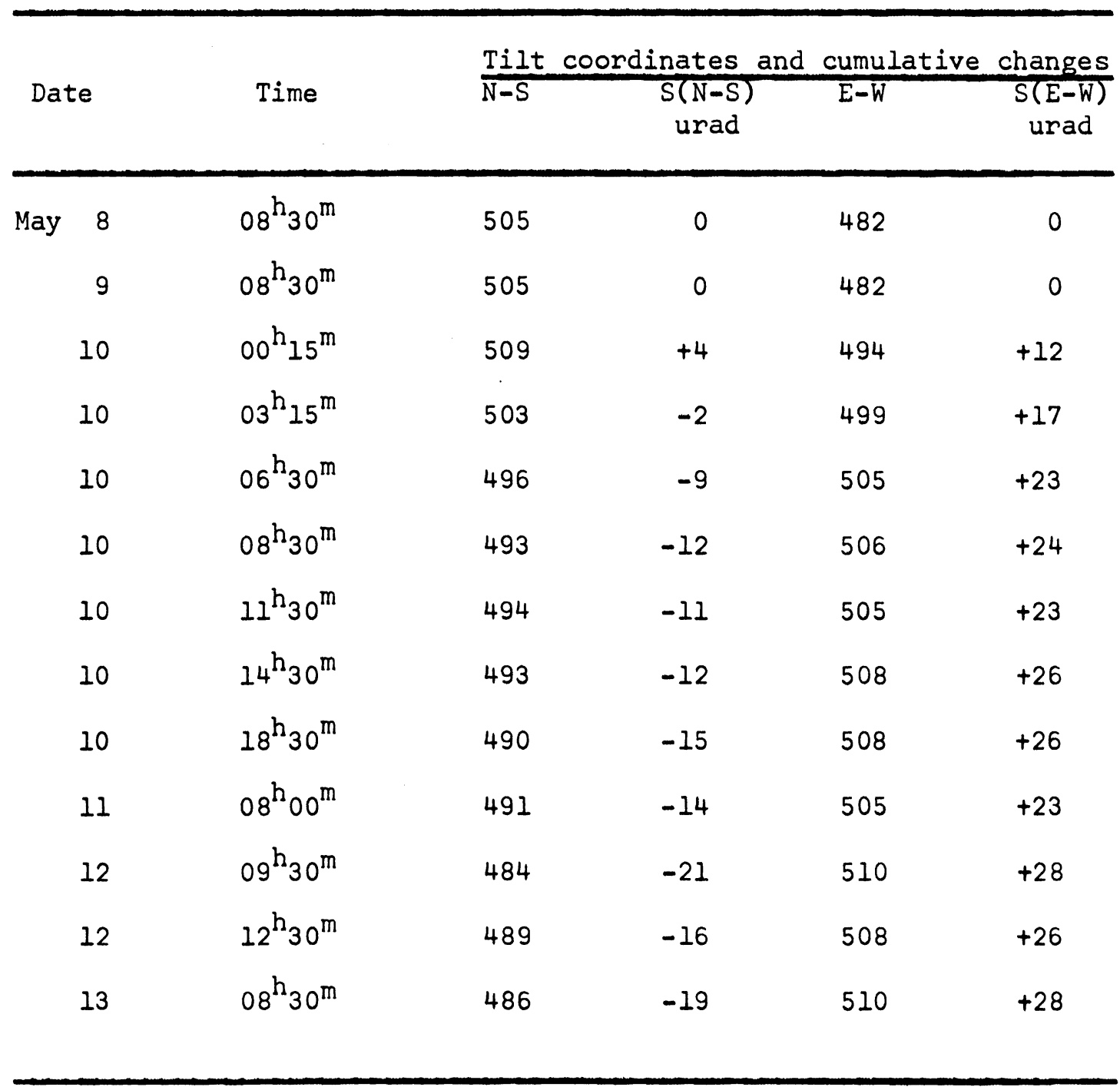




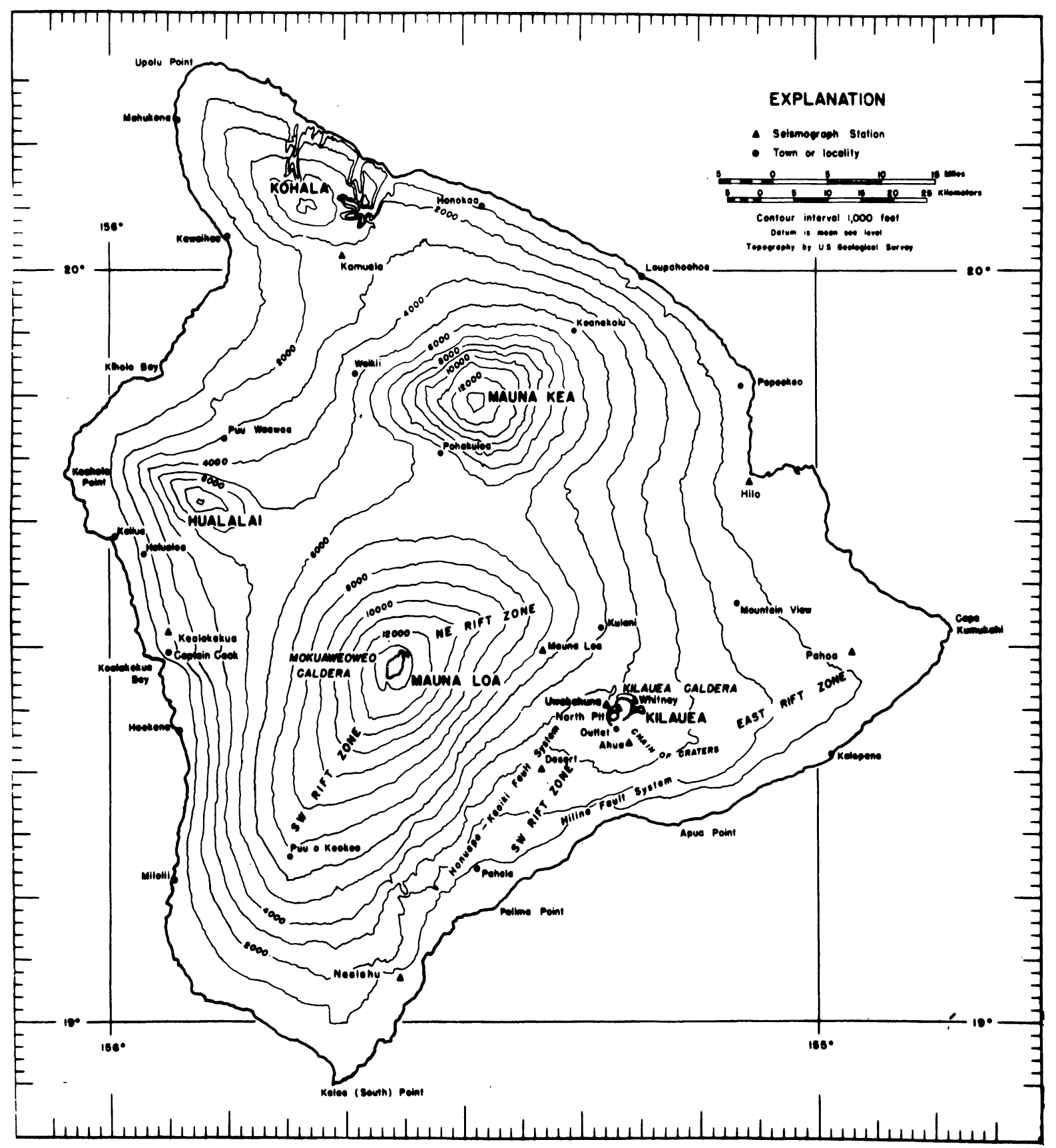

Figure 1.--Map of the island of Hawaii showing seismograph stations operated by the Geological Survey and localities mentioned in the text. Epicenters of earthquakes are given in terms of geographic coordinates, which are indicated at the edges of the map. 
Tilting of the ground around Kilauea caldera.- Tilting of the ground around the summit of Kilauea is monitored daily by a short-base water-tube tiltmeter in Uwekahuna Vault, and at irregular intervals it is measured on a regional scale by means of a network of field tilt-bases and a portable water-tube tiltmeter. The attitude of the ground surface at each tilt base is reported in terms of north-south and east-west tilt coordinates. Both coordinates at each station were arbitrarily set equal to 500 when measurements at that station were begun: Increasing tilt coordinates correspond to northward and eastward tilting of the earth ${ }^{\circ} s$ surface; $i_{0} e_{0}$, to a relative subsidence toward the north and east. A one-unit change in coordinate corresponds to a tilting of 1 microradian ( $1 \mathrm{~mm}$ per $\mathrm{km}$ ) in the direction indicated。

Table $10--$ Tilt coordinates at Uwekahuna Vault, April, May, and June 1963

\begin{tabular}{|c|c|c|c|c|c|c|c|}
\hline \multicolumn{2}{|c|}{ Date } & \multirow{2}{*}{$\frac{N \infty S}{495}$} & \multirow{2}{*}{$\frac{E-W}{482}$} & \multicolumn{2}{|c|}{ Date } & \multirow{2}{*}{$\frac{N-S}{504}$} & \multirow{2}{*}{$\frac{E-W}{496}$} \\
\hline April & 7 & & & June & 2 & & \\
\hline & 14 & 496 & 481 & & 9 & 507 & 494 \\
\hline & 21 & 490 & 483 & & 16 & 507 & 493 \\
\hline & 28 & 491 & 483 & & 23 & 507 & 493 \\
\hline \multirow[t]{4}{*}{ May } & 5 & 501 & 483 & & 30 & 505 & 497 \\
\hline & 12 & 491 & 504 & & & & \\
\hline & 19 & 494 & 502 & & & & \\
\hline & 26 & 501 & 497 & & & & \\
\hline
\end{tabular}


Table 2. - Pilt coordinates and changes at bases around Kilauea caldera (see fig. 2)

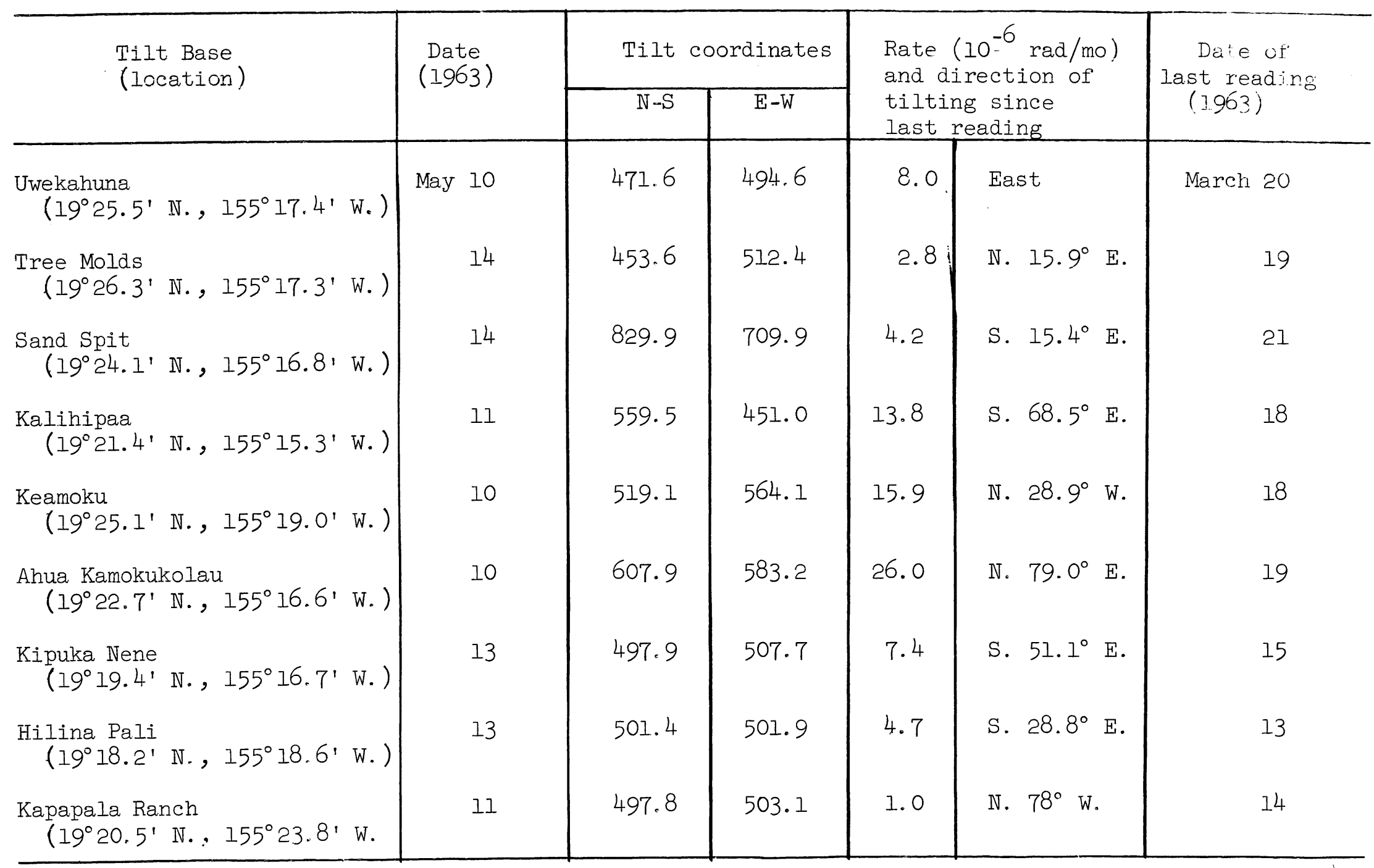




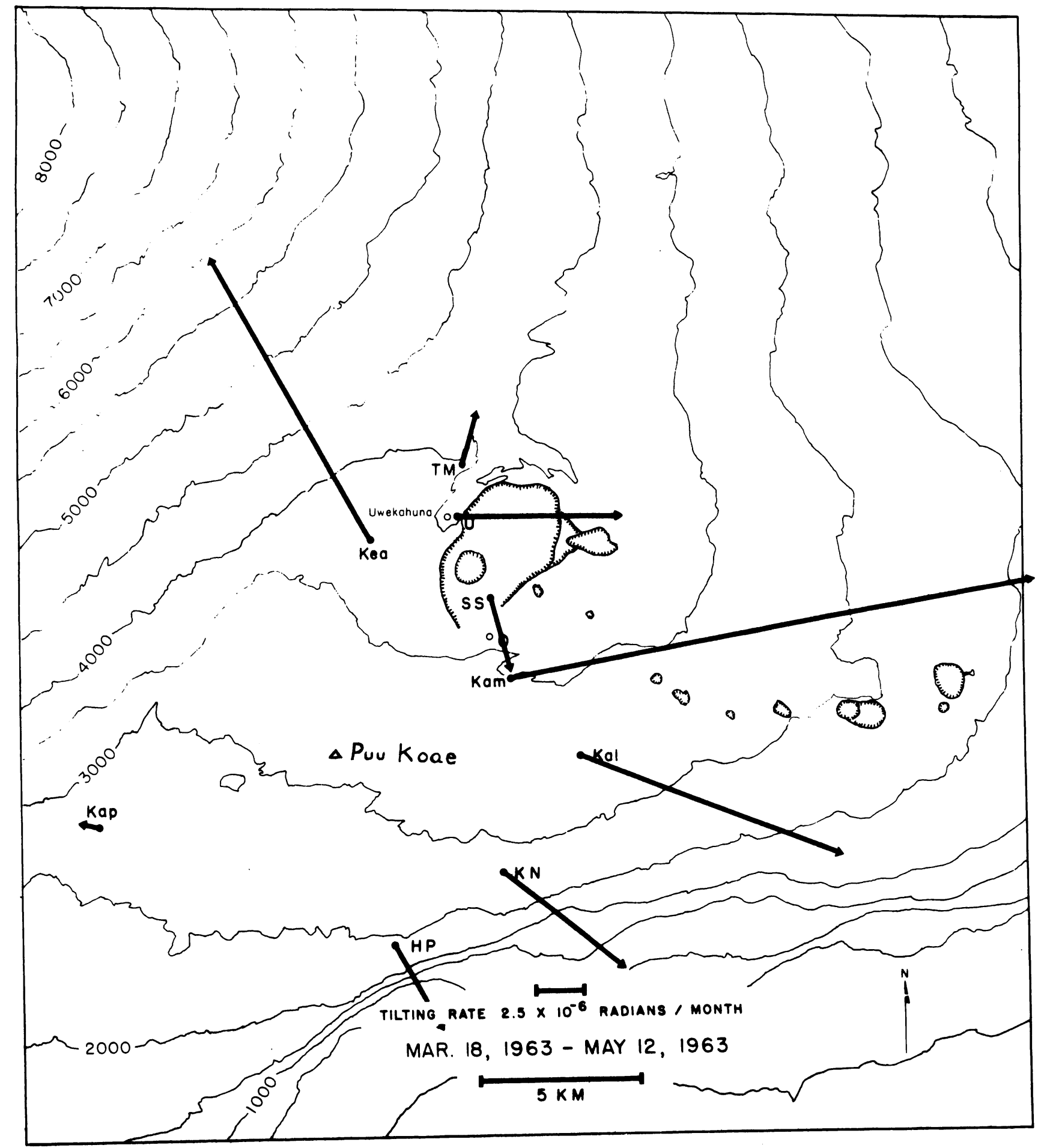

Figure 2.--Tilting of the ground around Kilauea caldera, March 18 to May 12, 1963. The vector depicting tilting at a given tilt-base points in the direction of maximum relative subsidence and has a length proportional to the rate of tilting during the measurement interval. Closed circles represent field tilt-bases; open circles, short-base water-tube tiltmeters. 
Seismic summary. --Events recorded by the U.S. Geological Survey seismograph network in Hawaii fall into two categories: local earthquakes and tremor originating in the region of the Hawaiian Islands, usually within $100 \mathrm{~km}$ of at least one seismograph, and distant earthquakes originating more than $3,000 \mathrm{~km}$ from Hawaii. As an index of seismic activity at Hawaiian volcanoes, daily counts of earthquakes and minutes of tremor recorded by seismographs in Hawaii are listed in table 3 . The earthquakes are separated into groups on the basis of region of origin as determined by analysis of records obtained daily at the Observatory (U, M, A, D, N, MP). Earthquakes of magnitude 2.5 or greater are generally sufficiently well recorded to be located with greater precision; they are listed individually in table 4. Data on identifiable phases from distant earthquakes are listed in table 5 .

Locations of the seismograph stations are shown on figure 1 , and essential data on the stations were given in Summary 29. 
Table 3. - Number of earthquakes and minutes of tremor recorded on seismographs

\section{$\mathrm{U}, \mathrm{M}, \mathrm{A}, \mathrm{D}, \mathrm{N}$, and $\mathrm{MP}$ around Kilauea caldera}

Tremor is separated into three categories: deep, intermediate, and shallow, on the basis of relative amplitudes on seismographs in the summit region. Unless otherwise stated, tremor is presumed to be associated with movement of magma within the central complex of Kilauea.

Earthquake categories are: Halemaumau rock slides, which are detected by the characteristic record they produce on the North Pit seismograph; shallow earthquakes in the Kilauea caldera region; shallow earthquakes along the SW. rift zone of Kilauea and the adjacent portion of the Kaoiki fault system; earthquakes along the eastern half of Kilauea's east rift zone (from the Pahoa seismograph); earthquakes from a source about $30 \mathrm{~km}$ beneath Halemaumau; earthquakes from the upper east rift zone and the adjacent fault systems of Kilauea's south flank, and earthquakes from other regions: Kona, Mauna Kea, etc. ?=Obscured by the swarm of earthquakes near Puu Koae.

\begin{tabular}{|c|c|c|c|c|c|c|c|c|c|c|}
\hline \multirow{2}{*}{$\begin{array}{l}\text { Date } \\
\text { (1963) }\end{array}$} & \multicolumn{3}{|c|}{$\begin{array}{c}\text { Tremor } \\
\text { (in minutes) }\end{array}$} & \multicolumn{7}{|c|}{ Earthquakes } \\
\hline & Deep & $\begin{array}{l}\text { Inter- } \\
\text { mediate }\end{array}$ & Shallow & $\begin{array}{l}\text { Hale- } \\
\text { maumau } \\
\text { slides }\end{array}$ & $\begin{array}{l}\text { Kilauea } \\
\text { caldera }\end{array}$ & $\begin{array}{l}\text { SW. rift } \\
\text { and } \\
\text { Kaoiki }\end{array}$ & $\begin{array}{c}\text { Eastern } \\
\text { East } \\
\text { rift } \\
\end{array}$ & $\begin{array}{l}\mathrm{Hale}- \\
\text { maumau } \\
30 \mathrm{~km}\end{array}$ & $\begin{array}{c}\text { Upper } \\
\text { East } \\
\text { rift } \\
\end{array}$ & Others \\
\hline $\begin{array}{r}\text { April } 1 \\
2 \\
3 \\
4 \\
5 \\
6 \\
7 \\
8 \\
9 \\
10 \\
11 \\
12 \\
13 \\
14 \\
15 \\
16\end{array}$ & 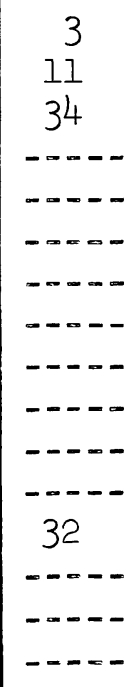 & 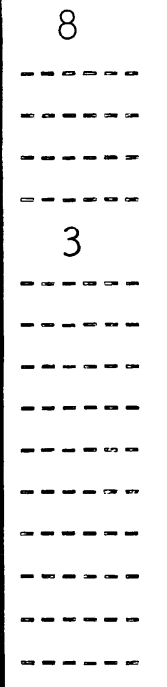 & 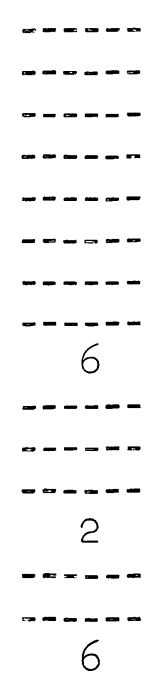 & 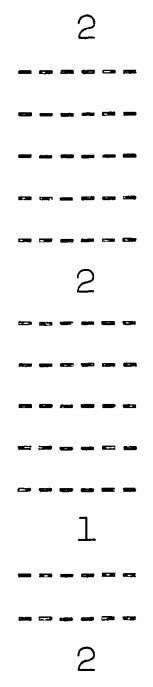 & $\begin{array}{r}170 \\
90 \\
90 \\
137 \\
150 \\
130 \\
160 \\
197 \\
195 \\
179 \\
196 \\
150 \\
115 \\
102 \\
70 \\
100\end{array}$ & $\begin{array}{r}11 \\
5 \\
13 \\
11 \\
15 \\
10 \\
13 \\
25 \\
10 \\
10 \\
18 \\
16 \\
5 \\
27 \\
29 \\
12\end{array}$ & 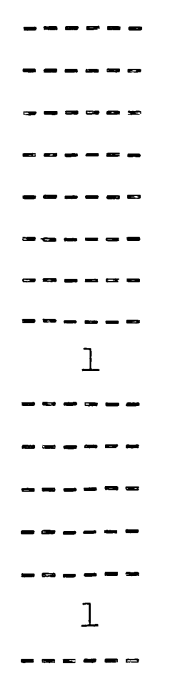 & $\begin{array}{c}4 \\
5 \\
4 \\
2 \\
3 \\
1 \\
3 \\
5 \\
- \\
- \\
6 \\
2 \\
2 \\
2 \\
4 \\
3 \\
2\end{array}$ & \begin{tabular}{c}
13 \\
10 \\
2 \\
\hdashline \\
0 \\
1 \\
5 \\
3 \\
6 \\
2 \\
6 \\
$\cdots$
\end{tabular} & I offshore Kona \\
\hline
\end{tabular}


Table 3.--Number of earthquakes and minutes of tremor recorded on seismographs

$\mathrm{U}, \mathrm{M}, \mathrm{A}, \mathrm{D}, \mathrm{N}$, and $\mathrm{MP}$ around Kilauea caldera--Continued

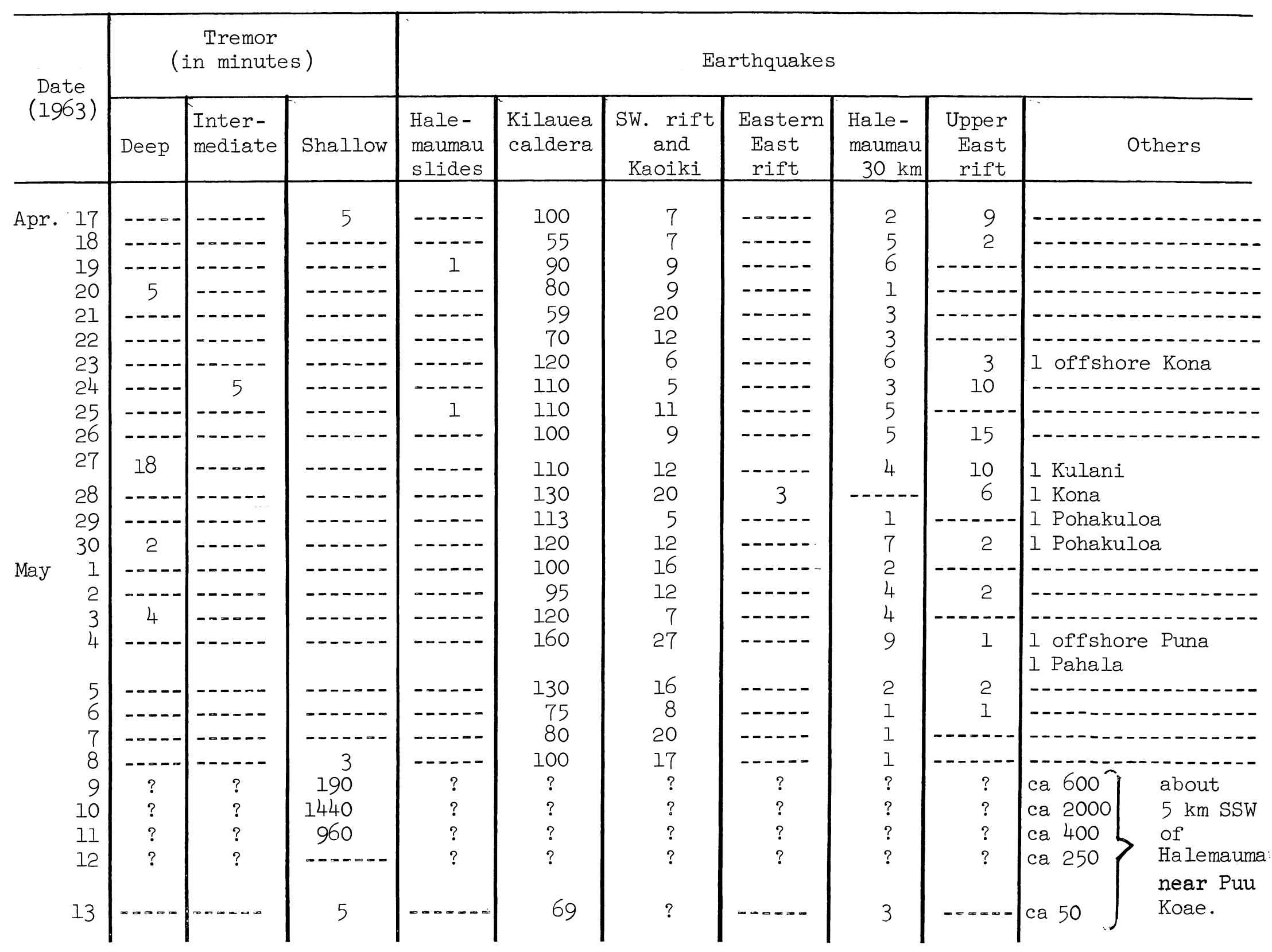


Table 3. - Number of earthquakes and minutes of tremor recorded on seismographs

U, M, A, D, N, and MP around Kilauea caldera--Continued

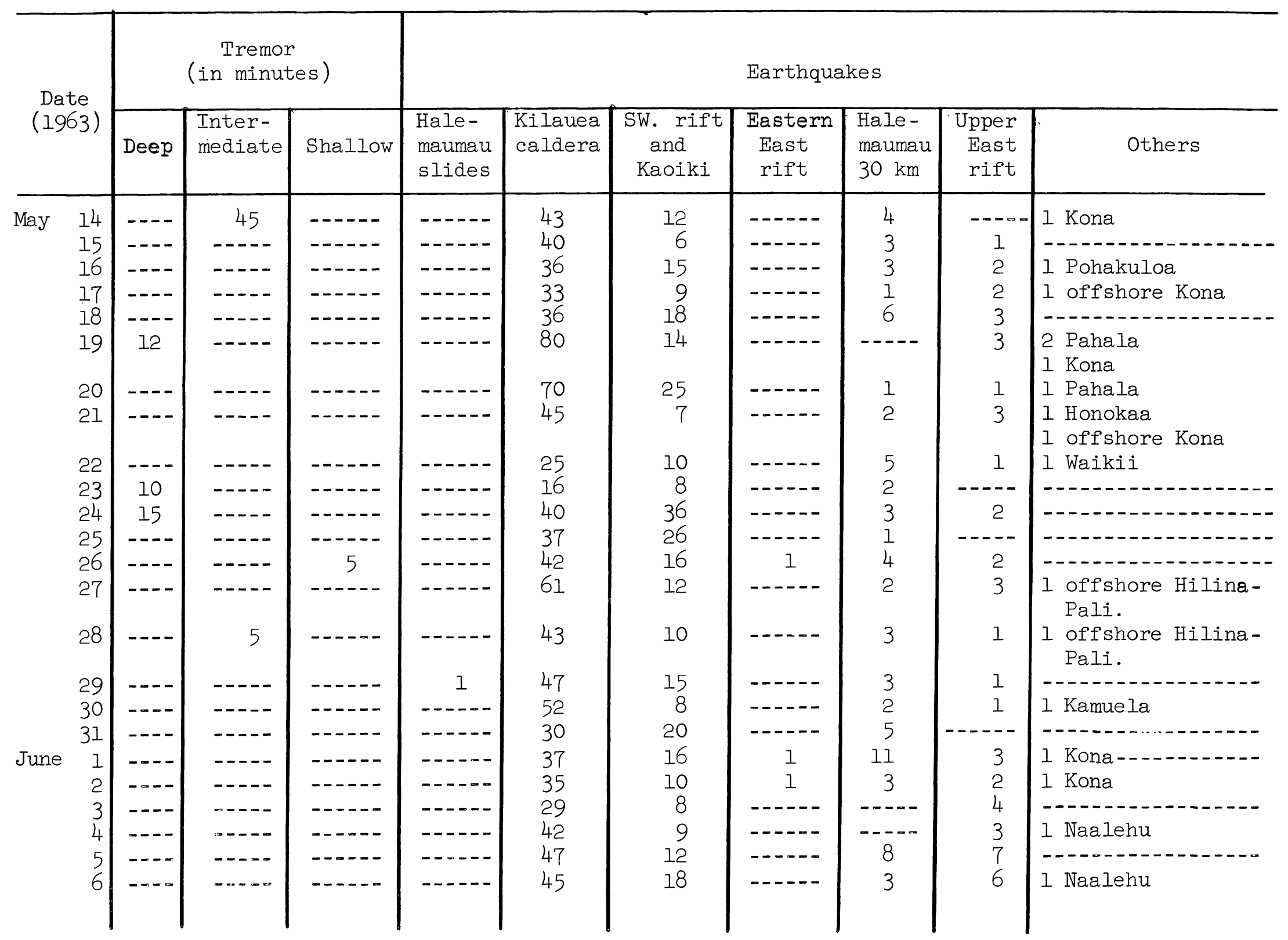


Table 3.--Number of earthquakes and minutes of tremor recorded on seismographs

\section{$\mathrm{U}, \mathrm{M}, \mathrm{A}, \mathrm{D}, \mathrm{N}$, and $\mathrm{MP}$ around Kilauea caldera--Continued}

\begin{tabular}{|c|c|c|c|c|c|c|c|c|c|c|}
\hline $\begin{array}{c}\text { Date } \\
(1963)\end{array}$ & \multicolumn{3}{|c|}{$\begin{array}{c}\text { Tremor } \\
\text { (in minutes) }\end{array}$} & \multicolumn{7}{|c|}{ Earthquakes } \\
\hline $\begin{array}{r}\text { June } 7 \\
8 \\
9 \\
10 \\
11 \\
12 \\
13 \\
14 \\
15 \\
16 \\
17 \\
18 \\
19 \\
20 \\
21 \\
22 \\
23 \\
24 \\
25 \\
26 \\
27 \\
28 \\
\\
29 \\
30\end{array}$ & 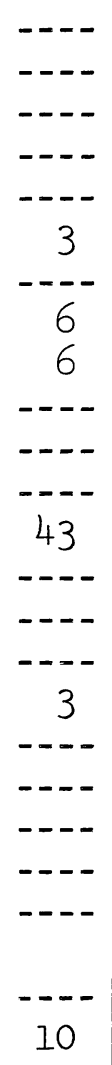 & 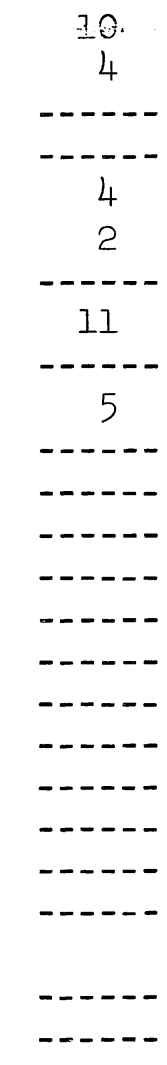 & 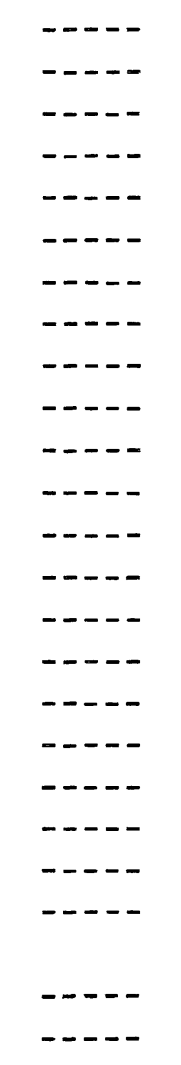 & 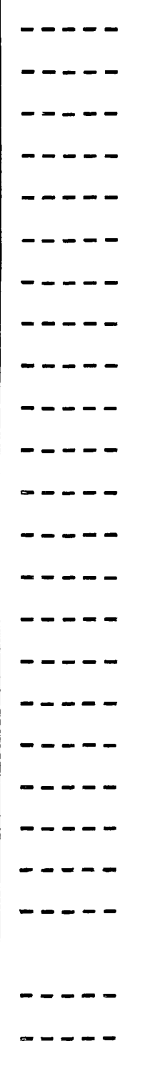 & $\begin{array}{l}60 \\
65 \\
65 \\
75 \\
51 \\
65 \\
68 \\
48 \\
66 \\
68 \\
40 \\
31 \\
42 \\
35 \\
34 \\
43 \\
48 \\
60 \\
46 \\
43 \\
26 \\
52 \\
87 \\
77 .\end{array}$ & $\begin{array}{r}12 \\
13 \\
10 \\
21 \\
8 \\
15 \\
18 \\
12 \\
15 \\
10 \\
10 \\
8 \\
3 \\
7 \\
8 \\
8 \\
11 \\
14 \\
9 \\
10 \\
3 \\
6 \\
8 \\
8\end{array}$ & 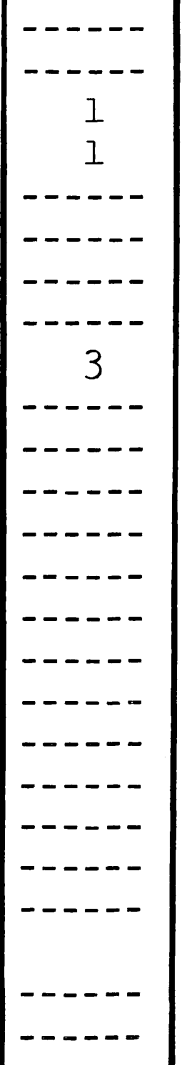 & $\begin{array}{r}9 \\
3 \\
3 \\
3 \\
2 \\
12 \\
8 \\
20 \\
2 \\
7 \\
5 \\
3 \\
1 \\
2 \\
6 \\
8 \\
15 \\
7 \\
6 \\
4 \\
2 \\
1 \\
\\
3 \\
3\end{array}$ & $\begin{array}{r}5 \\
10 \\
10 \\
15 \\
21 \\
20 \\
27 \\
17 \\
18 \\
11 \\
15 \\
8 \\
18 \\
11 \\
9 \\
7 \\
6 \\
6 \\
9 \\
25 \\
2 \\
--- \\
\\
45 \\
21\end{array}$ & $\begin{array}{l}\text { I Kona } \\
\text { I Kona }\end{array}$ Kona \\
\hline
\end{tabular}


Table 4.--Local earthquakes recorded by seismographs of the U.S. Geological Survey,

\section{April, May, and June 1963}

[Entries for a given quake are: date, origin time (Hawaiian Standard Time), magnitude, depth, epicenter, and felt reports. All earthquakes of magnitude 2.5 and larger, as well as many favorably located smaller ones, occurring on or near the island of Hawaii are included in the list.

In the following list, some origin times are followed only by "KM 30" and a statement of magnitude, These are all members of a continuing family of quakes noted also in other Summaries. The best

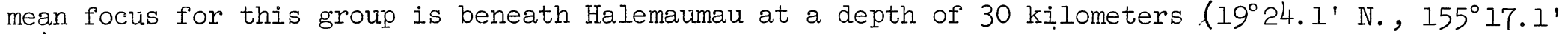
W. ).

In Summary 29, a persistent earthquake sequence was codified by the initials KT which referred to a "poor" location along the Kalapana Trail. This designation is retained for the purposes of this Summary but will be discontinued in the future unless Kalapana Trail quakes resume. The approximate epicenter for these quakes is $19^{\circ} 20^{\prime} \mathrm{N}$., and $155^{\circ} 05^{\prime} \mathrm{W}$., and shallow depth is assumed.

In Summary 24, "Kaoiki" was introduced as a symbol for listing any of a family of quakes with mean focus $19^{\circ} 24^{\prime}$ N., $155^{\circ} 25^{\prime} \mathrm{W} ., \mathrm{h}=3$ to $8 \mathrm{~km}$. This symbol is used in the following list]

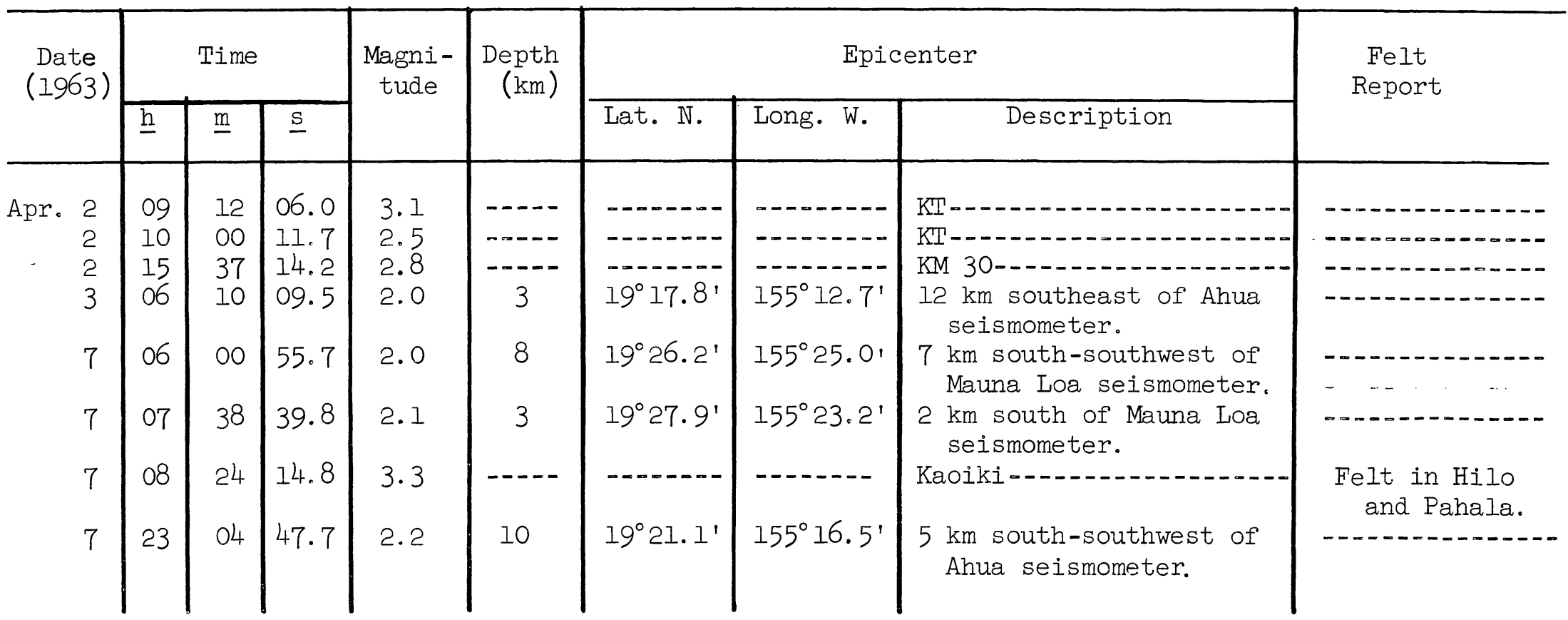


Table 4. --Local earthquakes recorded by seismographs of the U.S. Geological Survey,

April, May, and June 1963 --Continued

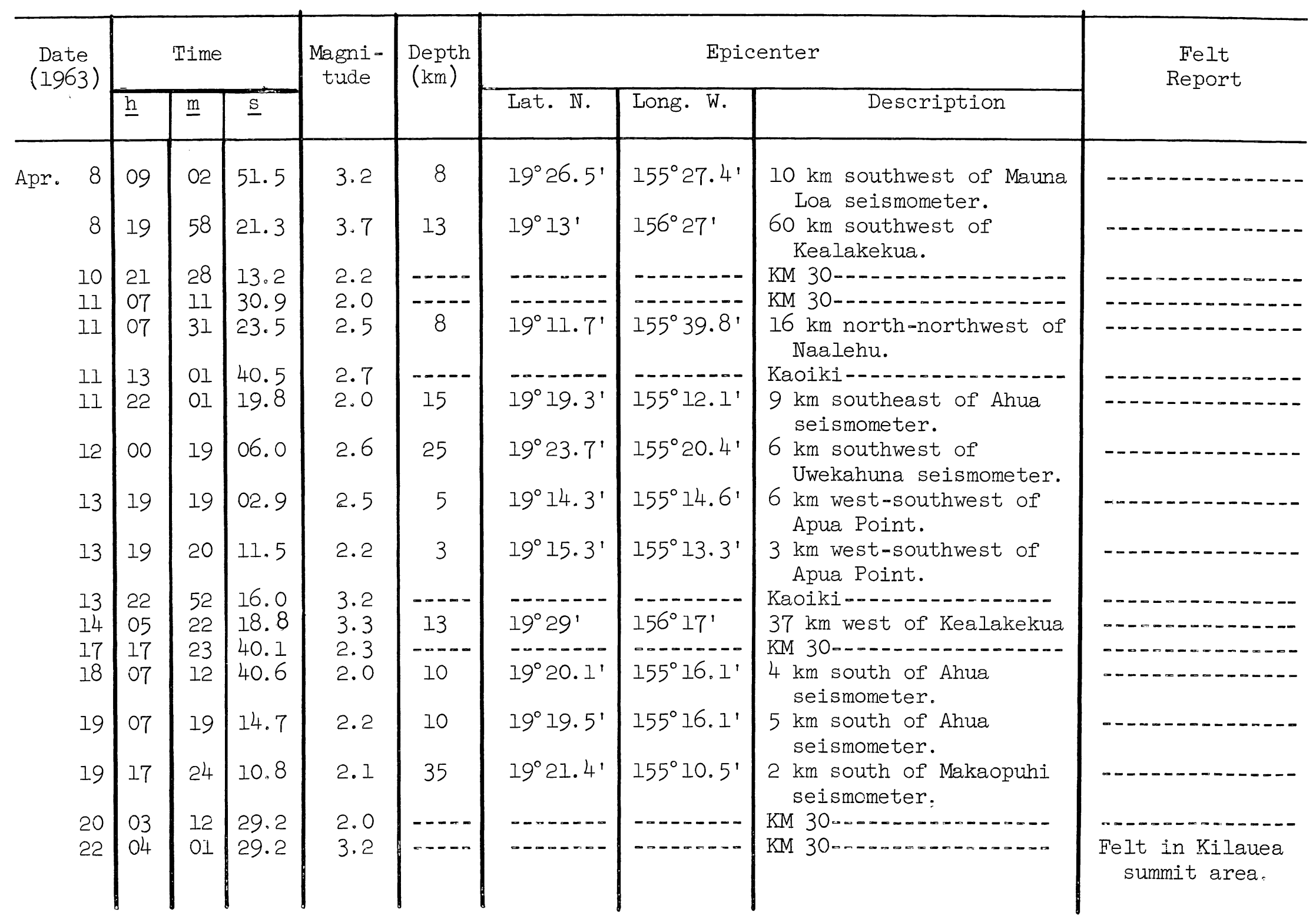


Table 4. - - Local earthquakes recorded by seismographs of the U.S. Geological Survey,

Apri., May; and Tune 1963--Continued

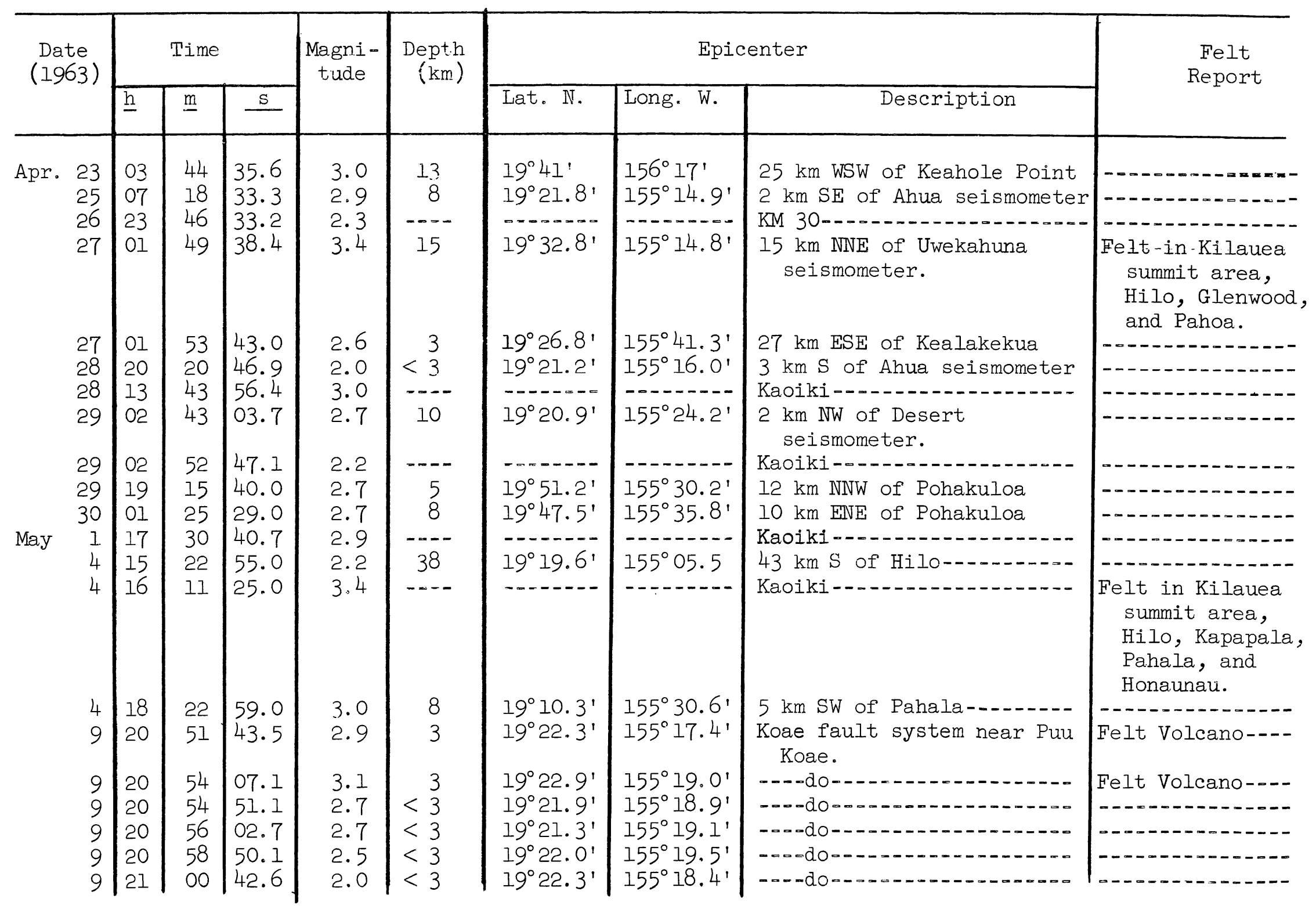


Table 4.--Local earthquakes recorded by seismographs of the U.S. Geological Survey,

April, May, and June 1963--Continued

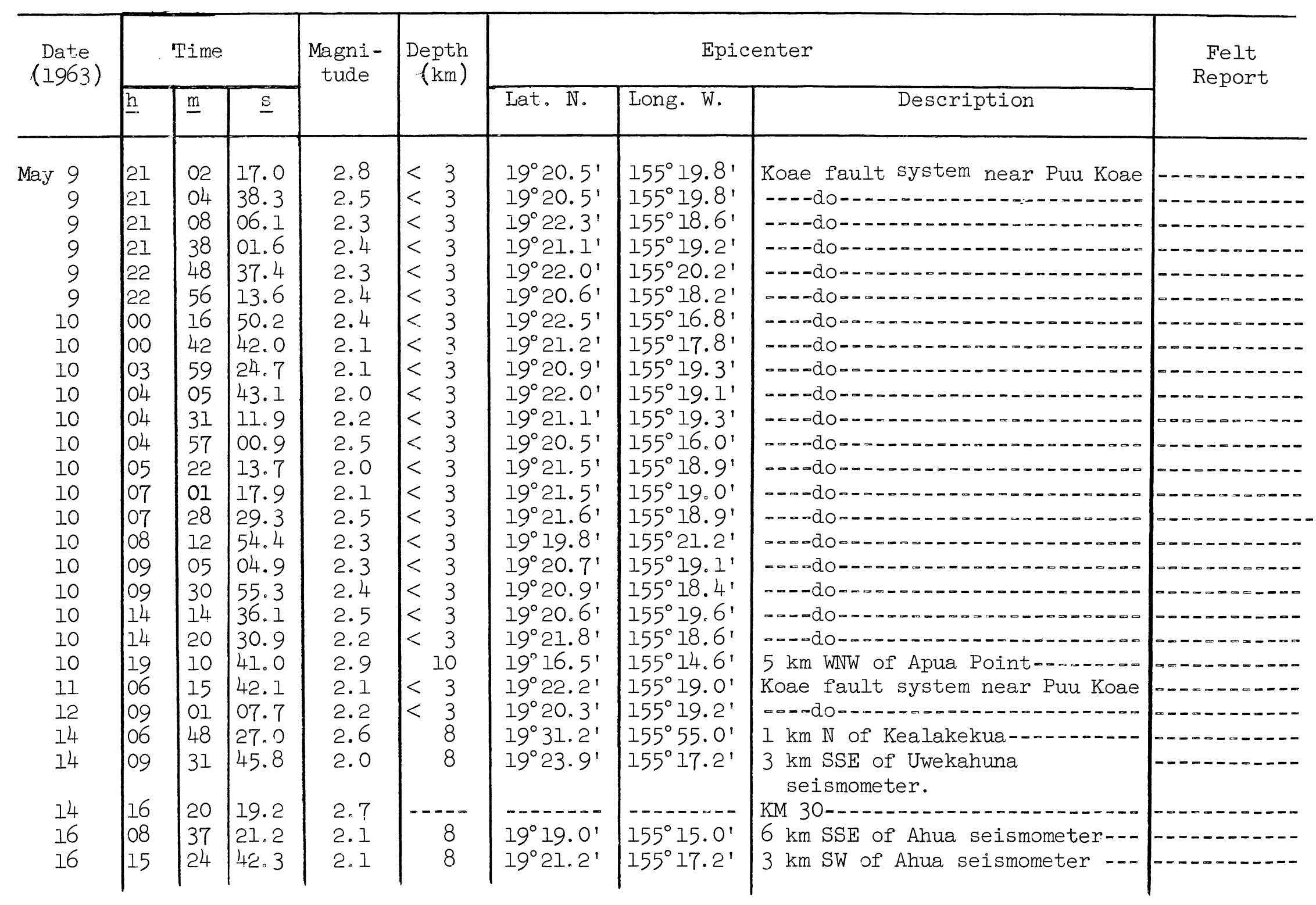


Table 4.--Local earthquakes recorded by seismographs of the U.S. Geological Survey, Apri.1, May, and June 1963--Continued

\begin{tabular}{|c|c|c|c|c|c|c|c|c|c|}
\hline \multirow{2}{*}{$\begin{array}{l}\text { Date } \\
\text { (1963) }\end{array}$} & \multicolumn{3}{|c|}{ Time } & \multirow{2}{*}{$\begin{array}{l}\text { Magni- } \\
\text { tude }\end{array}$} & \multirow{2}{*}{$\begin{array}{r}\text { Depth } \\
(\mathrm{km})\end{array}$} & \multicolumn{3}{|r|}{ Epicenter } & \multirow{2}{*}{$\begin{array}{c}\text { Felt } \\
\text { Report }\end{array}$} \\
\hline & $\underline{h}$ & $\underline{\mathrm{m}}$ & $\underline{S}$ & & & Iat. No & Long. W. & Description & \\
\hline May 16 & 21 & 22 & 41.5 & 2.1 & 5 & $19^{c} 46.5^{\prime}$ & $155^{\circ} 36.6$ & $10 \mathrm{~km}$ WNW of Pohakuloanomen & 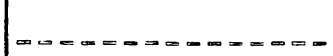 \\
\hline 17 & 01 & 31 & 19.0 & 2.8 & 8 & $19^{\circ} 36^{\circ}$ & $156^{\circ} 26^{\prime}$ & $57 \mathrm{~km}$ WNW of Kealakekua $=0=0$ & $0=-\infty-\infty-\infty-\infty-\infty)$ \\
\hline 17 & 09 & 19 & 07.0 & 2.5 & 20 & $19^{\circ} 23.5^{\prime}$ & $\left.155^{\circ}\right] 7.5^{\prime}$ & $3 \mathrm{~km} \mathrm{NW}$ of Ahua seismometer & 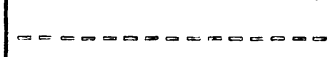 \\
\hline 17 & 04 & 54 & 16.9 & 2.2 & 25 & $19^{\circ} 12 \cdot 3^{\prime}$ & $155^{\circ} 32.2^{\prime}$ & $4 \mathrm{~km}$ west of Pahala $\ldots \ldots \ldots \ldots$ & $=--\infty--\infty=--1-\infty$ \\
\hline 19 & 01 & 54 & 59.4 & 3.4 & 5 & $19^{\circ} 12.4^{\prime}$ & $155^{\circ} 21.3^{\prime}$ & $14 \mathrm{~km}$ east of Pahala & $=-\infty=-\infty-\infty-\infty-\infty-\infty)$ \\
\hline 19 & 02 & 21 & 25.5 & 3.9 & 8 & $19^{\circ} 14.7^{i}$ & $155^{\circ} 31.1^{\prime}$ & $6 \mathrm{~km}$ NW of Pahala, & $\begin{array}{l}\text { Felt in Kilauea } \\
\text { summit area, } \\
\text { Hilo, Pahala, } \\
\text { and Waiohinu. }\end{array}$ \\
\hline 1.9 & 03 & 01 & $37 \cdot 7$ & 2.2 & $-\infty=-$ & $0-\infty-\infty-\infty$ & $----\infty-\infty$ & 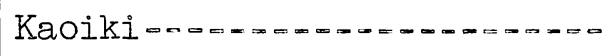 & $-\infty=\infty \ldots+\infty \ldots$ \\
\hline 19 & 17 & 20 & 08.6 & 3.3 & $--\infty$ & $-\infty-\infty-\infty-\infty$ & $-\infty-\infty-\infty-\infty$ & 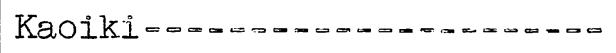 & 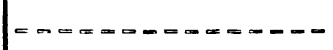 \\
\hline 19 & 19 & 37 & 36.2 & 2.0 & $-\infty=-$ & $-\infty-\infty+\infty-\infty$ & $-----\infty--\infty$ & 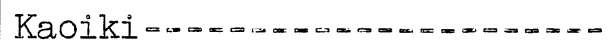 & $0-\infty-\infty-\infty-0,-\infty$ \\
\hline 19 & 19 & 56 & 10.1 & 2.9 & $=-\infty \infty$ & $-\infty-\infty-\infty)$ & $-m-\infty-\infty-\infty$ & 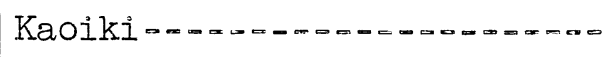 & $=0-\infty, 0=0-\infty-\infty-\ldots$ \\
\hline 19 & 22 & 06 & 23.9 & 2.5 & 8 & $19^{\circ} 41^{\prime}$ & $156^{\circ} 08^{\prime}$ & $10 \mathrm{~km} \mathrm{SW}$ of Keahole Point--- & $0=-\infty+\infty-\infty-\infty-\infty-\infty$ \\
\hline 20 & 03 & 09 & 52.1 & 2.2 & $=-\infty$ & $-\infty-\infty-\infty-\infty)$ & $-\infty=-\infty=-\infty-\infty$ & 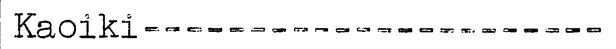 & 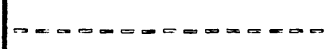 \\
\hline 20 & 04 & 11 & 16.0 & 2.8 & 8 & $19^{\circ} 14.3^{8}$ & $155^{\circ} 31.6^{\prime}$ & $7 \mathrm{~km} \mathrm{NW}$ of Pahala $\ldots \ldots \ldots \ldots$ & Felt in Pahala \\
\hline 20 & 10 & 31 & $27 \cdot 3$ & 3.1 & $=-\infty$ & $-\infty-\infty=-\infty$ & $-\infty=-\infty-0=0$ & 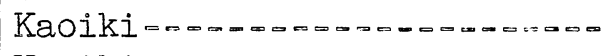 & $0=-\infty-0--\infty-\infty, \ldots-\infty$ \\
\hline 20 & 10 & 31 & 54.6 & 3.9 & $a=-\infty$ & $=-\infty-\infty-\infty$ & $-\infty-\infty-\infty-\infty$ & 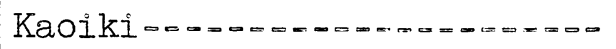 & 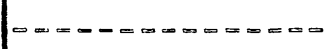 \\
\hline 20 & 10 & 39 & 28.7 & 2.1 & $\infty$ & $-\infty=\cos x=-\infty$ & $-\infty-\infty-\infty=0$ & 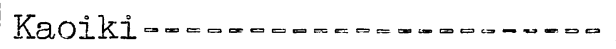 & $0000-000000-00$ \\
\hline 20 & 12 & 15 & 01.0 & 2.8 & 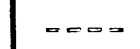 & $-\infty-\infty=-\infty=0$ & $-0-\infty-\infty=-\infty$ & Kaoiki & $\mid-\infty-\infty-\infty-\infty-\infty=\infty-\infty$ \\
\hline 21 & 06 & 19 & 26.4 & 2.4 & 3 & $19^{\circ} 17.2^{:}$ & $155^{\circ} 06.1^{\prime}$ & $\begin{array}{l}12 \mathrm{~km} \mathrm{SE} \text { of Makacpuhi } \\
\text { seismometer. }\end{array}$ & $-\infty-\ldots+\infty-\infty=-\infty \ldots$ \\
\hline $2 \bar{i}$ & 09 & 25 & 59.2 & 2.0 & $=\infty-\infty$ & $-\infty-\infty-\infty$ & $-\infty-\infty-\infty \infty-\infty$ & 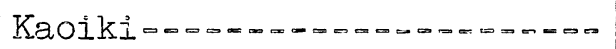 & $00=\infty=-\infty-\infty \infty-\infty,-\infty$ \\
\hline 21 & 14 & 26 & 55.3 & 2.6 & 8 & $19^{\circ} 54.2^{\prime}$ & $155^{\circ} 29.0^{\prime}$ & $18 \mathrm{~km} \mathrm{~S}$ of Honokaa $=\ldots \ldots$ & 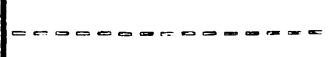 \\
\hline 21 & 19 & 27 & 09.7 & $2 \cdot 3$ & 3 & $19^{\circ} 08.0^{\circ}$ & $155^{\circ} 16.8^{\prime}$ & $\begin{array}{l}16 \mathrm{~km} \mathrm{SE} \text { of Desert } \\
\text { seismometer. }\end{array}$ & $-\cdots \infty-\infty \cdots \infty-\infty$ \\
\hline 21 & 20 & 15 & 19.1 & 2.5 & 13 & $19^{\circ} 39^{\prime}$ & $156^{\circ} 27^{\prime}$ & $42 \mathrm{~km}$ WSW of Keahole Point & $-\infty-2-\infty+\infty=-\infty=-1$ \\
\hline 22 & 06 & 07 & 57.7 & 2.8 & 45 & $19^{\circ} 16.9^{\prime}$ & $155^{\circ} \mathrm{O} 1.5^{\prime}$ & $25 \mathrm{~km} \mathrm{SW}$ of Pahoan.......... & 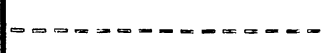 \\
\hline 22 & 23 & 01 & 20.5 & 2.6 & 3 & $19^{\circ} 52.8^{\prime}$ & $155^{\circ} 40.6^{\prime}$ & 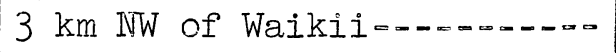 & 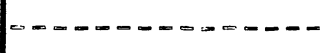 \\
\hline 23 & 10 & 34 & 32.5 & 2.5 & 5 & $19^{\circ} 14.2^{\prime}$ & $155^{\circ} 22.1^{\prime}$ & $\begin{array}{l}12 \mathrm{~km} \text { SSE of Desert } \\
\text { seismometer. }\end{array}$ & $=\infty \rightarrow \infty-\infty-\infty-\infty-\infty-\infty$ \\
\hline
\end{tabular}


Table 4. - -Local earthquakes recorded by seismographs of the U.S. Geological Survey, April, May, and June 1963--Continued

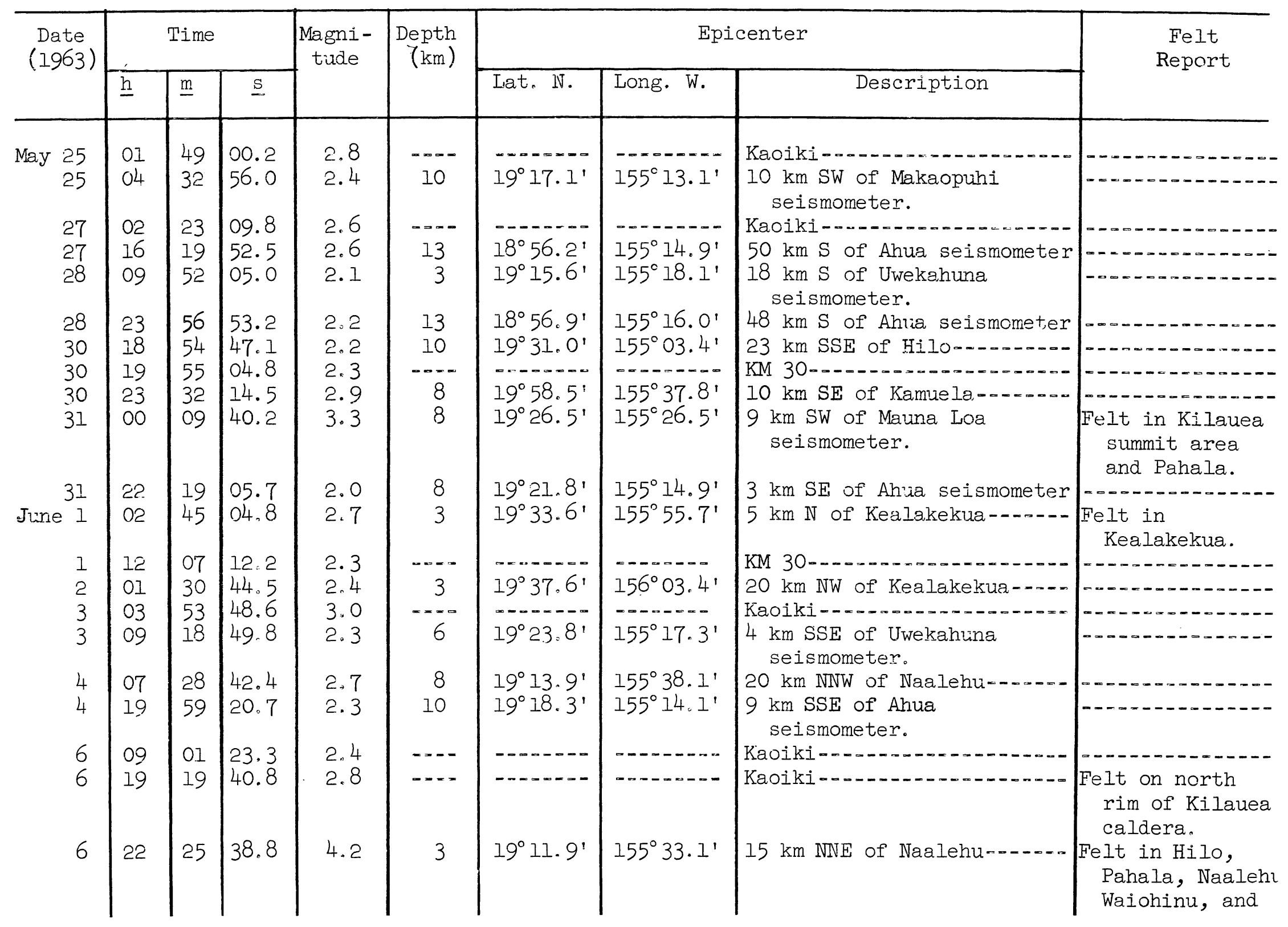


Table 4.--Local earthquakes recorded by seismographs of the U.S. Geological Survey,

April, May, and June 1963--Continued

\begin{tabular}{|c|c|c|c|c|c|c|c|c|c|}
\hline \multirow{2}{*}{$\begin{array}{l}\text { Date } \\
(1963)\end{array}$} & \multicolumn{3}{|c|}{ Time } & \multirow{2}{*}{$\begin{array}{l}\text { Magni- } \\
\text { tiude }\end{array}$} & \multirow{2}{*}{$\begin{array}{r}\text { Depth } \\
(\mathrm{km})\end{array}$} & \multicolumn{3}{|c|}{ Epicenter } & \multirow{2}{*}{$\begin{array}{l}\text { Felt } \\
\text { Report }\end{array}$} \\
\hline & h & $\underline{\mathrm{m}}$ & $\underline{s}$ & & & Lat: $\mathrm{N}$. & Long. W. & Description & \\
\hline June 7 & 12 & 52. & 25.6 & 2.4 & 15 & $19^{\circ} 23.4^{\prime}$ & $155^{\circ} 59.5^{\prime}$ & $13 \mathrm{~km} \mathrm{SW}$ of Pahoa & 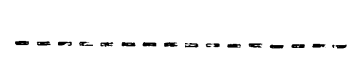 \\
\hline & 22 & 04 & 05.7 & 2.2 & 40 & $19^{\circ} 18.8^{\prime}$ & $155^{\circ} 08.4^{\prime}$ & $\begin{array}{l}7 \mathrm{~km} \text { SW of Makaopuhi } \\
\text { seismometer. }\end{array}$ & 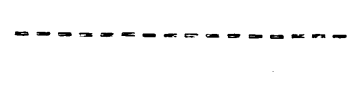 \\
\hline 8 & 03 & 49 & 06.1 & 2.9 & $---\infty$ & $-\infty-\infty-\infty$ & $------\infty$ & Kaoiki $\ldots \ldots \ldots$ & $=-\infty--\infty---n-\infty-n$ \\
\hline 9 & 05 & 03 & 28.7 & 2.6 & $<3$ & $19^{\circ} 25.3^{:}$ & $155^{\circ} 45.5^{\prime}$ & $15 \mathrm{~km}$ ENE of Hookena......- & 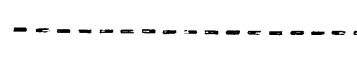 \\
\hline 10 & 20 & 49 & 51.8 & 2.4 & 8 & $19^{\circ} 22.8^{\prime}$ & $155^{\circ} 15.1^{\prime}$ & I km NE of Ahua seismometer & $=-\infty--\infty--\infty-\infty-\infty=-$ \\
\hline 10 & 21 & 00 & 15.5 & 2.6 & 8 & $19^{\circ} 22.6^{\prime}$ & $155^{\circ} 14.5^{\prime}$ & $\begin{array}{l}3 \mathrm{~km} \text { ENE of Ahua } \\
\text { seismometer. }\end{array}$ & 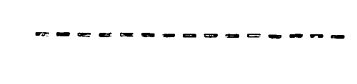 \\
\hline 12 & 03 & 50 & 01.7 & 2.2 & 8 & $19^{\circ} 20.1^{\prime}$ & $155^{\circ} 09.0^{\prime}$ & $\begin{array}{l}4 \mathrm{~km} \mathrm{SE} \text { of Makaopuhi } \\
\text { seismometer. }\end{array}$ & 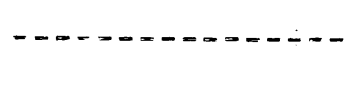 \\
\hline 12 & 07 & 58 & 32.0 & 2.4 & $<3$ & $19^{\circ} 28.7^{\prime}$ & $155^{\circ} 46.8^{\prime}$ & $15 \mathrm{~km}$ ESE of Kealakekua... & 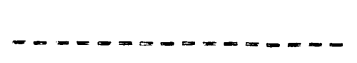 \\
\hline 12 & 11 & 49 & 56.6 & 2.0 & 8 & $19^{\circ} 20.4^{\prime}$ & $155^{\circ} 08.3^{\prime}$ & $\begin{array}{l}5 \mathrm{~km} \text { SE of Makaopuhi } \\
\text { seismometer. }\end{array}$ & $--\infty=--\infty-\infty=-\infty-\infty$ \\
\hline 13 & 07 & 28 & 15.7 & 2.4 & $-\infty-\infty$ & $-\infty-\cdots-\cdots$ & $-\infty-\cdots---$ & KM $30-\cdots \ldots \ldots \ldots \ldots \ldots \ldots n$ & 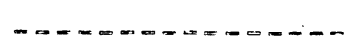 \\
\hline 13 & 07 & 32 & 50.3 & 2.4 & $=-\infty$ & $-\cdots--\cdots$ & $-x-\infty---n$ & KM $30 \ldots \ldots$ & $-\infty--\infty+\infty-\infty-\infty-n$ \\
\hline 14 & 11 & 17 & 57.0 & 3.7 & 30 & $19^{\circ} 26.2^{\prime}$ & $155^{\circ} 16.7^{\prime}$ & $\begin{array}{l}2 \mathrm{~km} \mathrm{NE} \text { of Uwekahuna } \\
\text { seismometer. }\end{array}$ & $\begin{array}{l}\text { Felt in Kilauea } \\
\text { summit area, } \\
\text { Kulani, ard } \\
\text { north Kona. }\end{array}$ \\
\hline .14 & 11 & 43 & 57.8 & 2.4 & 25 & $19^{\circ} 26.1^{\prime}$ & $155^{\circ} 17.3^{\prime}$ & $\begin{array}{l}\text { I } \mathrm{km} \mathrm{NE} \text { of Uwekahuna } \\
\text { seismometer. }\end{array}$ & 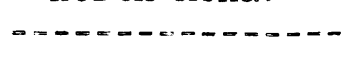 \\
\hline 14 & 11 & 45 & 37.0 & 2.3 & 25 & $19^{\circ} 26.1^{\prime}$ & $155^{\circ} 17.1^{\prime}$ & $\begin{array}{l}\text { I } \mathrm{km} \mathrm{NE} \text { of Uwekahuna } \\
\text { seismometer. }\end{array}$ & $-\cdots=--\infty, \ldots-n$ \\
\hline 14 & 12 & 46 & 38.4 & 2.4 & 25 & $19^{\circ} 25.9^{\prime}$ & $155^{\circ} 16.4^{\prime}$ & $\begin{array}{l}2 \mathrm{~km} \mathrm{NE} \text { of Uwekahuna } \\
\text { seismometer. }\end{array}$ & $-\infty-\ldots-\ldots-n-\infty$ \\
\hline 16 & 06 & 31 & 35.6 & 2.2 & 3 & $19^{\circ} 20.5^{\prime}$ & $155^{\circ} 49.3^{\prime}$ & $10 \mathrm{~km} \mathrm{SE}$ of Hookena & $-\infty-\infty-\infty----n$ \\
\hline 17 & 04 & 14 & 35.2 & 2.4 & $<3$ & $19^{\circ} 34.2^{\prime}$ & $155^{\circ} 53.3^{\prime}$ & $7 \mathrm{~km}$ NNE of Kealakekua & $-\infty-n-\infty-\infty-\infty-\infty$ \\
\hline 17 & 04 & 36 & 19.0 & 2.4 & 13 & $19^{\circ} 21.7^{\prime}$ & $155^{\circ} 06.2^{!}$ & $\begin{array}{l}9 \mathrm{~km} \mathrm{E} \text { of Makaopuhi } \\
\text { seismometer. }\end{array}$ & $=--\infty---\infty-\infty--\infty$ \\
\hline 17 & 14 & 48 & 42.9 & 2.0 & 5 & $19^{\circ} 18.6^{\prime}$ & $155^{\circ} 14.4^{\prime}$ & $\begin{array}{l}8 \mathrm{~km} \text { SSE of Ahua } \\
\text { seismometer. }\end{array}$ & $=-\infty=-----\infty=-\infty$ \\
\hline
\end{tabular}


Table 4. =-Iocal earthquakes recorded by seismographs of the U.S. Geological Survey, April, May, and June 1963--Continued

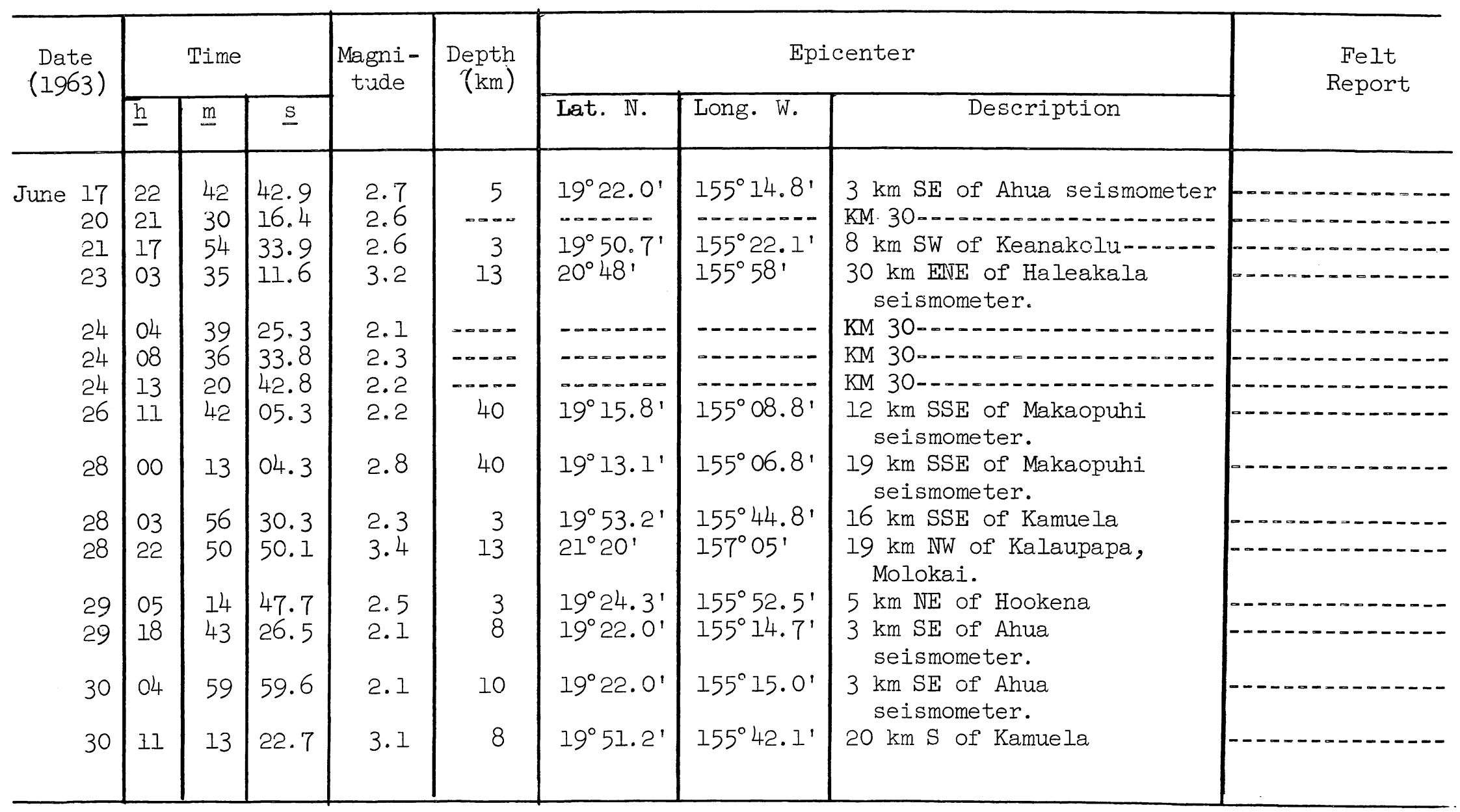


Table 5.--Distant earthquakes

[Times are reported in Greenwich Civil Time which is 10 hours faster than Hawaiian Standard Time. A " $c$ " following the time of $\mathrm{P}$ indicates compressional first motion; a "d" indicates dilatational first motion. Station symbols, locations, and instrumentation were presented in summary 29. Magnitudes calculated from the Hawaii seismograms are followed by (HVO). Location of epicenters, origin times, focal depths, and magnitudes reported by other institutions are taken from "Preliminary Determination of Epicenters" published by the U.S. Coast and Geodetic Survey]

\section{April 2, 1963}

$\begin{array}{lllr}\text { M } & \text { Z } & \text { iP } & 16: 25: 43.5 \mathrm{~d} \\ \mathrm{~A} & \mathrm{Z} & \text { iP } & 44.5 \mathrm{~d} \\ \mathrm{U} & \mathrm{Z} & \text { iP } & 43.8 \mathrm{~d} \\ \mathrm{Ha} & \mathrm{Z} & \text { iP } & 31.7 \mathrm{c} \\ \mathrm{Ka} & \mathrm{Z} & \text { iP } & 35.8 \mathrm{~d} \\ \mathrm{Hi} & \text { Z } & \text { iP } & 42.7 \mathrm{~d} \\ \mathrm{Na} & \text { Z } & \text { iP } & 46.7 \mathrm{c}\end{array}$

C\&GS card 25-63:

$16: 18: 55.6$

$53.2^{\circ} \mathrm{N}, 171.7^{\circ} \mathrm{W}$.

Andreanof Islands, Aleutian Islands $\mathrm{h}$ about $142 \mathrm{~km}$

Magnitude 6.25-6.5 (Pas)

$$
\begin{aligned}
& 5.5(\mathrm{Brk}) \\
& 5.7 \text { (CGS). }
\end{aligned}
$$

\section{April 3}

$\begin{array}{llll}\mathrm{U} & \mathrm{PEZ} & \mathrm{eR} & 15: 23: 49 \\ \mathrm{~Pa} & \mathrm{Z} & \text { Tmax } & 16: 32: 32\end{array}$

C\&GS card 35-63:

$14: 47: 55.5$

$55.4^{\circ} \mathrm{S} ., 128.2^{\circ} \mathrm{W}$.

South Pacific Ocean

$\mathrm{h}$ about $33 \mathrm{~km}$

Magnitude 5.8 (CGS).

\section{April 6}

$\begin{array}{lll}\mathrm{M} & \mathrm{Z} & i \mathrm{P} \\ \mathrm{A} & \mathrm{Z} & \mathrm{iP} \\ \mathrm{D} & \mathrm{Z} & \mathrm{eP} \\ \mathrm{U} & \mathrm{Z} & \mathrm{iP} \\ \mathrm{Pa} & \mathrm{Z} & \mathrm{iP} \\ \mathrm{Hi} & \mathrm{Z} & \mathrm{iP} \\ \mathrm{Ka} & \mathrm{Z} & i \mathrm{P} \\ \mathrm{Ha} & \mathrm{Z} & i P\end{array}$

April 6--Continued

C\&GS card 30-63:

$07: 03: 06.5$

$17.5^{\circ}$ S., $178.9^{\circ} \mathrm{W}$.

Fiji Islands region

$h$ about $526 \mathrm{~km}$

Magnitude 5.1 (CGS).

\section{April 6}

$\begin{array}{lllrl}M & Z & \text { iP } & 11: 27: 28.7 & \mathrm{~d} \\ \mathrm{~A} & \mathrm{Z} & \text { iP } & 29.0 \mathrm{~d} \\ \mathrm{D} & \mathrm{Z} & \text { iP } & 29.3 \mathrm{~d} \\ \mathrm{U} & \mathrm{Z} & \text { eP } & 28.5 \mathrm{c}\end{array}$

C\&GS card 27-63:

$11: 19: 23.3$

$63.4^{\circ} \mathrm{N} ., 149.5^{\circ} \mathrm{W}$.

Central Alaska

$\mathrm{h}$ about $39 \mathrm{~km}$

Magnitude 5.5 (CGS).

\section{April 9}

$\begin{array}{lllr}M & Z & \text { iP } & 02: 09: 43.3 \mathrm{~d} \\ \mathrm{~A} & \mathrm{Z} & \mathrm{eP} & 42.8 \mathrm{~d} \\ \mathrm{U} & \mathrm{Z} & \text { iP } & 43.0 \mathrm{~d} \\ \mathrm{Na} & \mathrm{Z} & \text { iP } & 39.0 \mathrm{c} \\ \mathrm{Pa} & \mathrm{Z} & \mathrm{eP} & 43.3 \mathrm{c} \\ \mathrm{Hi} & \mathrm{Z} & \text { iP } & 44.1 \mathrm{c} \\ \mathrm{Ha} & \text { Z } & \text { iP } & 47.8 \mathrm{c}\end{array}$

C\&GS card 30-63:

$02: 02: 25.1$

$17.7^{\circ}$ S., $178.7^{\circ} \mathrm{W}$.

Fiji Islands region

$h$ about $538 \mathrm{~km}$

Magnitude 4.9 (CGS). 
Table 5. --Distant earthquakes--Continued

\section{April 10, 1963}

$\begin{array}{lllr}\text { M } & \text { Z } & \text { iP } & 08: 02: 57.6 \mathrm{c} \\ \mathrm{A} & \mathrm{Z} & \text { iP } & 57.4 \mathrm{~d} \\ \mathrm{D} & \mathrm{Z} & \mathrm{eP} & 56.1 \mathrm{c} \\ \mathrm{U} & \mathrm{Z} & \text { iP } & 57.3 \mathrm{~d} \\ \mathrm{Na} & \mathrm{Z} & \mathrm{eP} & 55.4 \mathrm{c} \\ \mathrm{Ha} & \text { Z } & \text { iP } & 08: 03: 00.6 \mathrm{c}\end{array}$

C\&GS card 31-63:

$07: 50: 30.2$

$9.2^{\circ} \mathrm{S.}, 125.0^{\circ} \mathrm{E}$.

Timor

$\mathrm{h}$ about $33 \mathrm{~km}$

Magnitude 5.2 (CGS).

\section{April 13}

\begin{tabular}{|c|c|c|c|}
\hline $\mathrm{M}$ & Z & $e P$ & $02: 33: 04.3$ \\
\hline$A$ & Z & iP & 03.7 \\
\hline A & Z & $i p P$ & $33.9 \mathrm{c}$ \\
\hline D & Z & $\mathrm{eP}$ & 03.9 c \\
\hline $\mathrm{U}$ & Z & $\mathrm{eP}$ & $03.8 c$ \\
\hline $\mathrm{U}$ & Z & epP & 33.9 \\
\hline $\mathrm{Na}$ & Z & $\mathrm{eP}$ & $04.6 c$ \\
\hline $\mathrm{Pa}$ & Z & $\mathrm{eP}$ & 02.1 \\
\hline $\mathrm{Hi}$ & Z & iP & 03.2 \\
\hline $\mathrm{Ka}$ & Z & $\mathrm{eP}$ & 05.9 \\
\hline $\mathrm{Ha}$ & Z & iP & 10.8 \\
\hline $\mathrm{U}$ & PEZ & iP & 04 \\
\hline U & PEZ & $i p P$ & 36 \\
\hline U & PEZ & iPS & $02: 44: 26$ \\
\hline \multirow[t]{3}{*}{$\mathrm{U}$} & PEN & is & $02: 43: 08$ \\
\hline & & iSS & $02: 48: 40$ \\
\hline & & $i G$ & $02: 54: 54$ \\
\hline
\end{tabular}

C\&GS card 31-63:

$02: 20: 57.5$

$6.2^{\circ}$ S., $76.5^{\circ} \mathrm{W}$.

Central Peru

$\mathrm{h}$ about $125 \mathrm{~km}$

Magnitude 6.75-7 (Pas),

6.3 (CGS).

\section{April 13}

$\begin{array}{lll}\mathrm{M} & \mathrm{Z} & \text { iP } \\ \mathrm{A} & \mathrm{Z} & \text { iP } \\ \mathrm{D} & \mathrm{Z} & \mathrm{eP} \\ \mathrm{U} & \mathrm{Z} & \mathrm{eP} \\ \mathrm{Na} & \mathrm{Z} & \text { iP } \\ \mathrm{Pa} & \mathrm{Z} & \mathrm{eP}\end{array}$

$14: 42: 43.0 \mathrm{~d}$

$43.2 \mathrm{~d}$

$42.1 \mathrm{~d}$

$42.9 \mathrm{~d}$

$40.8 \mathrm{~d}$

$45.0 \mathrm{~d}$
April 13--Continued

$\mathrm{Hi} \quad \mathrm{Z}$ iP $\quad 45.0 \mathrm{~d}$

C\&GS card 30-63:

$14: 31: 21.0$

$3.4^{\circ} \mathrm{S} ., 135.4^{\circ} \mathrm{E}$.

Near north coast of New Guinea $h$ about $31 \mathrm{~km}$

Magnitude 5.6 (CGS).

\section{April 16}

\begin{tabular}{|c|c|c|c|}
\hline M & Z & $e P$ & $01: 41: 19.5 \mathrm{~d}$ \\
\hline A & Z & iP & $18.7 \mathrm{~d}$ \\
\hline D & Z & iP & 18.0 \\
\hline $\mathrm{U}$ & Z & iP & 19.4 \\
\hline $\mathrm{Pa}$ & Z & eP & 20.6 \\
\hline $\mathrm{Ha}$ & Z & $\mathrm{eP}$ & 23.4 \\
\hline $\mathrm{U}$ & PEZ & iP & 19 \\
\hline U & PEZ & iSS & $01: 56: 15$ \\
\hline U & PEZ & $i R$ & $02: 04: 51$ \\
\hline $\mathrm{U}$ & $\mathrm{PEE}$ & is & $01: 51: 17$ \\
\hline $\mathrm{U}$ & $\mathrm{PEE}$ & iPPS & $01: 52: 00$ \\
\hline $\mathrm{U}$ & PEN & iSSS & $01: 59: 24$ \\
\hline & & $i G$ & $02: 01: 21$ \\
\hline
\end{tabular}

C\&GS card 31-63:

$01: 29: 19.4$

$0.8^{\circ} \mathrm{S.}, 128.0^{\circ} \mathrm{E}$.

Halmahera region

$\mathrm{h}$ about $33 \mathrm{~km}$

Magnitude 7 (Pas), 6.1 (CGS), 7.5 (HVO).

Apri1 16

$\begin{array}{lllr}M & Z & \text { iP } & 01: 48: 55.2 \\ \mathrm{~A} & \mathrm{Z} & \text { iP } & 54.6 \mathrm{c} \\ \mathrm{D} & \mathrm{Z} & \text { iP } & 53.5 \mathrm{c} \\ \mathrm{Pa} & \mathrm{Z} & \mathrm{eP} & 56.1 \mathrm{~d} \\ \mathrm{Ha} & \mathrm{Z} & \mathrm{eP} & 59.1 \mathrm{c} \\ \mathrm{U} & \mathrm{PEZ} & \text { iS } & 01: 59: 01 \\ \mathrm{U} & \mathrm{PEZ} & \text { iR } & 02: 12: 29 \\ \mathrm{U} & \text { PEN } & \text { iSS } & 02: 04: 17\end{array}$

C\&GS card 31-63:

$01: 36: 59.4$

$1.2^{\circ}$ S., $128.4^{\circ} \mathrm{E}$.

Halmahera region

$\mathrm{h}$ about $33 \mathrm{~km}$

Magnitude 6.3 (CGS), 7.5 (HVO). 
Table 5.--Distant earthquakes--Continued

April 16, 1963

$\begin{array}{lllr}\text { A } & \text { Z } & \text { iP } & 02: 07: 13.9 \mathrm{c} \\ \text { D } & \text { Z } & \text { eP } & 12.6 \mathrm{c} \\ \text { U } & \text { Z } & \text { iP } & 14.3 \mathrm{c} \\ \text { Ha } & \text { Z } & \text { eP } & 18.0 \mathrm{c} \\ \text { U } & \text { PEE } & \text { ISS } & 02: 22: 01 \\ \text { U } & \text { PEN } & \text { ISSS } & 02: 25: 28 \\ \text { U } & \text { PEN } & \text { iG } & 02: 27: 11 \\ \text { U } & \text { PEZ } & \text { iR } & 02: 31: 04\end{array}$

C\&GS card 31-63:

$01: 55: 10.9$

$0.7^{\circ}$ S., $128.0^{\circ} \mathrm{E}$.

Halmahera region

$\mathrm{h}$ about $32 \mathrm{~km}$

Magnitude 6.0 (CGS),

$$
7.25 \text { (HVO). }
$$

\section{April 16}

$\begin{array}{lllr}\text { M } & \text { Z } & \text { iP } & 02: 17: 51.0 \mathrm{c} \\ \mathrm{A} & \mathrm{Z} & \mathrm{eP} & 50.3 \mathrm{c} \\ \mathrm{D} & \mathrm{Z} & \mathrm{eP} & 49.3 \mathrm{c} \\ \mathrm{U} & \mathrm{Z} & \mathrm{eP} & 51.1 \mathrm{c}\end{array}$

C\&GS card 31-63:

$02: 05: 42$

$1.3^{\circ} \mathrm{S} ., 126.9^{\circ} \mathrm{E}$.

Halmahera region

Magnitude 5.8 (CGS).

\section{April 17}

$\begin{array}{lllc}\mathrm{Pa} & \mathrm{Z} & \text { iP } & 02: 19: 58.0 \mathrm{~d} \\ \mathrm{~A} & \mathrm{Z} & \text { iP } & 57.0 \mathrm{~d} \\ \mathrm{~N} & \mathrm{Z} & \text { iP } & 57.5 \mathrm{~d} \\ \mathrm{U} & \mathrm{PEZ} & \text { iS } & 02: 26: 43 \\ \mathrm{U} & \mathrm{PEZ} & \text { iR } & 02: 32: 53 \\ \mathrm{U} & \text { PEN } & \text { iG } & 02: 30: 27\end{array}$

C\&CSS card 31-63:

$02: 11: 26.1$

$19.6^{\circ} \mathrm{S} ., 178.6^{\circ} \mathrm{E}$.

Fiji Islands

$\mathrm{h}$ about $33 \mathrm{~km}$

Magnitude 6.5-6.75 (Pas),

$$
\begin{aligned}
& 6.0 \text { (Brk), } \\
& 5.9 \text { (CGS), } \\
& 5.9 \text { (HVO). }
\end{aligned}
$$

\section{April 19}

$\begin{array}{llll}\text { U } & \text { PEN } & \text { IS } & 07: 59: 22 \\ \text { U } & \text { PEN } & \text { IG } & 08: 14: 16 \\ U & \text { PEZ } & \text { IPS } & 08: 00: 53 \\ \text { U } & \text { PEZ } & \text { iSS } & 08: 05: 44 \\ \text { U } & \text { PEZ } & \text { iL } & 08: 13: 16 \\ \text { U } & \text { PEZ } & \text { iR } & 08: 18: 06\end{array}$

C\&GS card 32-63:

$07: 35: 23.7$

$35.8^{\circ}$ N., $96.9^{\circ} \mathrm{E}$.

Tsinghai Province, China

$\mathrm{h}$ about $33 \mathrm{~km}$

Magnitude 7 (Pas), 6.75-7 (Brk), 6.1 (CGS), 7 (HVO).

April 24

$\begin{array}{lllrl}M & Z & \text { iP } & 21: 50: 23.7 & \mathrm{c} \\ \mathrm{A} & \mathrm{Z} & \text { eP } & 23.1 \mathrm{c} \\ \mathrm{N} & \mathrm{Z} & \text { iP } & 23.2 \mathrm{c} \\ \mathrm{MP} & \mathrm{Z} & \text { iP } & 23.0 \mathrm{c} \\ \mathrm{Ha} & \text { Z } & \text { iP } & 28.3 \mathrm{c}\end{array}$

C\&GS card 33-63:

$21: 42: 49.0$

$20.8^{\circ} \mathrm{S}, 179.1^{\circ} \mathrm{W}$.

$\mathrm{Fiji}$ Islands region

$\mathrm{h}$ about $603 \mathrm{~km}$

Magnitude 5.1 (CGS).

April 25

$\begin{array}{lllr}\text { M } & \text { Z } & \text { iP } & 08: 24: 14.1 \mathrm{~d} \\ \mathrm{~A} & \mathrm{Z} & \text { iP } & 14.3 \mathrm{~d} \\ \mathrm{D} & \mathrm{Z} & \text { iP } & 13.6 \mathrm{~d} \\ \text { MP } & \text { Z } & \text { iP } & 14.5 \mathrm{~d} \\ \mathrm{U} & \mathrm{Z} & \text { iP } & 13.9 \mathrm{~d} \\ \mathrm{Ha} & \text { Z } & \text { iP } & 09.3 \mathrm{~d}\end{array}$

C\&GS card 33-63:

$08: 12: 57.2$

$4.7^{\circ}$ N., $122.4^{\circ} \mathrm{E}$.

Celebes Sea

$\mathrm{h}$ about $610 \mathrm{~km}$

Magnitude 5.5 (CGS). 
Table 5.--Distant earthquakes--Continued

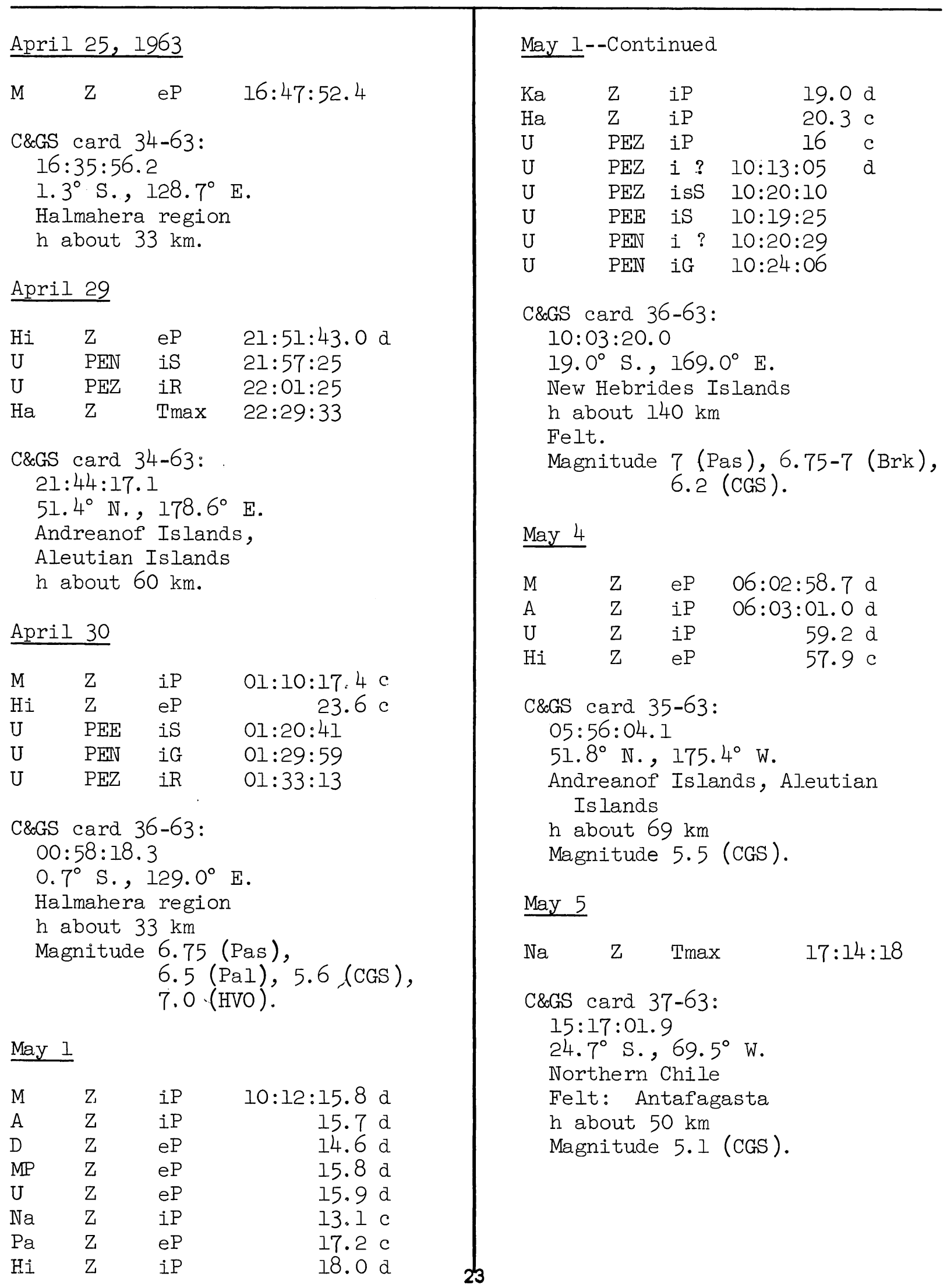


Table 5.--Distant earthquakes--Continued

\begin{tabular}{|c|c|c|c|}
\hline \multicolumn{4}{|c|}{ May 8, 1963} \\
\hline M & Z & iP & $08: 57: 48.6 \mathrm{c}$ \\
\hline M & Z & iPP & $08: 59: 16.2 \mathrm{c}$ \\
\hline A & Z & iP & $08: 57: 49.4 \mathrm{c}$ \\
\hline A & Z & ePP & $08: 59: 17.5 \mathrm{c}$ \\
\hline D & Z & iP & $08: 57: 50.4 \mathrm{c}$ \\
\hline D & Z & iPP & $08: 59: 17.2 \mathrm{c}$ \\
\hline $\mathrm{MP}$ & Z & iP & $08: 57: 49.7 \mathrm{c}$ \\
\hline MP & Z & ePP & $08: 59: 17.8 \mathrm{~d}$ \\
\hline $\mathrm{U}$ & Z & iP & $08: 57: 49.1 \mathrm{c}$ \\
\hline $\mathrm{U}$ & Z & ¿PP & $08: 59: 16.7 \mathrm{c}$ \\
\hline $\mathrm{Hi}$ & Z & iP & $08: 57: 47.5 \mathrm{c}$ \\
\hline $\mathrm{Hi}$ & Z & iPP & $08: 59: 13.8 \mathrm{c}$ \\
\hline $\mathrm{Pa}$ & Z & iP & $08: 57: 49.0 \mathrm{c}$ \\
\hline $\mathrm{Pa}$ & Z & ePP & $08: 59: 16.0 \mathrm{c}$ \\
\hline $\mathrm{Ha}$ & Z & iPP & $08: 58: 56.0 \mathrm{~d}$ \\
\hline M & Z & Tmax & $09: 35: 41$ \\
\hline$A$ & Z & Tmax & 33 \\
\hline D & Z & $T \max$ & 28 \\
\hline $\mathrm{MP}$ & Z & $T \max$ & 26 \\
\hline U & Z & $T \max$ & 24 \\
\hline $\mathrm{Pa}$ & Z & $T \max$ & 28 \\
\hline $\mathrm{Ka}$ & Z & $T \max$ & $09: 34: 36$ \\
\hline $\mathrm{Ha}$ & Z & $T \max$ & $09: 33: 46$ \\
\hline
\end{tabular}

C\&GS card 35-63:

$08: 50: 56.0$

$54.9^{\circ}$ N., $163.9^{\circ} \mathrm{W}$.

Unimak Island,

Aleutian Islands region

$\mathrm{h}$ about $89 \mathrm{~km}$

Magnitude 5.6 (CGS).

\section{May 8}

$\begin{array}{llll}\text { M } & \text { Z } & \text { eP } & 10: 31: 54.6 \mathrm{c} \\ \text { U } & \text { PEE } & \text { iS } & 10: 40: 00 \\ \text { U } & \text { PEE } & \text { iG } & 10: 46: 34 \\ \text { U } & \text { PEZ } & \text { eR } & 10: 49: 20\end{array}$

C\&GS card 35-63:

$10: 22: 11.2$

$36.6^{\circ} \mathrm{N} ., 141.0^{\circ} \mathrm{E}$.

Honshu, Japan

$\mathrm{h}$ about $53 \mathrm{~km}$

Magnitude 6.1 (CGS).

May 8

$\begin{array}{lllr}M & Z & \text { iP } & 15: 35: 55.4 \mathrm{~d} \\ \mathrm{~A} & \mathrm{Z} & \text { iP } & 55.5 \mathrm{~d} \\ \mathrm{~N} & \text { Z } & \text { iP } & 55.6 \mathrm{~d}\end{array}$

C8AGS card 40-63:

$15: 24: 00.3$

$5.3^{\circ}$ N., $125.7^{\circ} \mathrm{E}$.

Off coast of Mindanao,

Philippine Islands

$\mathrm{h}$ about $70 \mathrm{~km}$

Magnitude 5.6 (CGS).

May 12

\begin{tabular}{|c|c|c|c|}
\hline A & Z & iP & $20: 15: 53.5$ \\
\hline D & Z & iP & 53.7 \\
\hline MP & Z & iP & 53.7 \\
\hline U & Z & iP & 53.4 \\
\hline $\mathrm{Ha}$ & Z & iP & 47.6 \\
\hline $\mathrm{Hi}$ & Z & iP & 51.2 \\
\hline $\mathrm{Na}$ & Z & iP & 57.1 \\
\hline $\mathrm{U}$ & PEZ & iP & 54 \\
\hline U & PEZ & eS & $20: 21: 43$ \\
\hline $\mathrm{U}$ & PEZ & $i R$ & $20: 25: 52$ \\
\hline $\mathrm{Ha}$ & $\mathrm{Z}$ & $T \max$ & $20: 53: 44$ \\
\hline
\end{tabular}

C\&GS card 37-63:

$20: 08: 43.0$

$57.4^{\circ}$ N., $153.9^{\circ} \mathrm{W}$.

Kodiak Island, Alaska

$\mathrm{h}$ about $80 \mathrm{~km}$

Magnitude 5.9 (CGS), 5.8 (HVO).

May 14

$\begin{array}{lllrl}M & Z & \text { iP } & 15: 20: 13.2 & \mathrm{c} \\ \mathrm{A} & \mathrm{Z} & \mathrm{eP} & 13.0 \mathrm{c} \\ \mathrm{N} & \mathrm{Z} & \mathrm{eP} & 13.1 \mathrm{c} \\ \mathrm{MP} & \mathrm{Z} & \text { iP } & 13.5 \mathrm{c}\end{array}$

C\&GS card 42-63:

$15: 08: 46.1$

$5.6^{\circ} \mathrm{S} ., 127.8^{\circ} \mathrm{E}$.

Banda Sea

$\mathrm{h}$ about $405 \mathrm{~km}$

Magnitude 5.3 (CGS). 
Table 5.--Distant earthquakes--Continued

\section{May 17, 1963}

$\begin{array}{lllr}\text { M } & \text { Z } & \text { iP } & 07: 42: 18.2 \mathrm{c} \\ \mathrm{A} & \mathrm{Z} & \text { iP } & 18.0 \mathrm{c} \\ \mathrm{D} & \mathrm{Z} & \mathrm{eP} & 17.0 \mathrm{c} \\ \mathrm{MP} & \mathrm{Z} & \mathrm{eP} & 17.6 \mathrm{c} \\ \mathrm{U} & \mathrm{Z} & \mathrm{iP} & 18.1 \mathrm{c} \\ \mathrm{Ka} & \mathrm{Z} & \mathrm{eP} & 20.8 \mathrm{c}\end{array}$

C\&GS card 40-63:

$07: 33: 17.5$

$31.0^{\circ}$ S., $179.8^{\circ} \mathrm{W}$.

Kermadec Islands region

$\mathrm{h}$ about $358 \mathrm{~km}$

Magnitude 4.7 (CGS).

\section{May 17}

$\begin{array}{lll}\mathrm{M} & \mathrm{Z} & \text { iP } \\ \mathrm{A} & \mathrm{Z} & \mathrm{eP} \\ \mathrm{D} & \mathrm{Z} & \mathrm{eP} \\ \mathrm{U} & \mathrm{Z} & \mathrm{iP} \\ \mathrm{Hi} & \mathrm{Z} & \mathrm{iP} \\ \mathrm{Ka} & \mathrm{Z} & \mathrm{iP} \\ \mathrm{Ha} & \mathrm{Z} & \mathrm{iP}\end{array}$

$22: 48: 46.3 \mathrm{c}$ $45.7 \mathrm{c}$ $44.8 \mathrm{c}$ $45.9 \mathrm{c}$ $47.3 \mathrm{c}$ $48.8 \mathrm{c}$ $51.8 \mathrm{c}$

C\&GS card 41-63:

$22: 40: 06.7$

$24.4^{\circ} \mathrm{S} ., 177.2^{\circ} \mathrm{W}$.

Tonga Islands region

$\mathrm{h}$ about $70 \mathrm{~km}$

Magnitude 5.9 (CGS).

\section{May 19}

$\begin{array}{lllr}\text { U } & \text { PEZ } & \text { ePP } & 01: 20: 37 \\ \text { U } & \text { PEZ } & \text { ISS } & 01: 35: 01 \\ \text { U } & \text { PEZ } & \text { iR } & 01: 47: 57 \\ \text { U } & \text { PEE } & \text { IPS } & 01: 29: 31 \\ \text { U } & \text { PEE } & \text { IPPS } & 01: 30: 07 \\ \text { U } & \text { PEN } & \text { IS } & 01: 27: 26 \\ \text { U } & \text { PEN } & \text { eL } & 01: 42: 29 \\ \text { U } & \text { PEN } & \text { eG } & 01: 43: 30 \\ \text { M } & \text { Z } & \text { Tmax } & 03: 04: 35 \\ \text { A } & \text { Z } & \text { Tmax } & 31 \\ \text { D } & \text { Z } & \text { Tmax } & 33 \\ \text { MP } & \text { Z } & \text { Tmax } & 26 \\ \text { U } & \text { Z } & \text { Tmax } & 34 \\ \text { Pa } & \text { Z } & \text { Tmax } & 15 \\ \text { Na } & \text { Z } & \text { Tmax } & 23 \\ \text { Hi } & \text { Z } & \text { Tmax } & 27\end{array}$

May 19--Continued

C\&GS card 39-63:

$01: 03: 04.1$

$46.5^{\circ}$ S., $75.1^{\circ} \mathrm{W}$.

Coast of southerm Chile

$\mathrm{h}$ about $33 \mathrm{~km}$

Magnitude 6.75 (Pas), 6.5 (CGS), 7 (HVO).

\section{May 20}

\begin{tabular}{|c|c|c|c|}
\hline M & Z & iP & $11: 47: 27.8 \mathrm{~d}$ \\
\hline A & Z & iP & $27.4 \mathrm{~d}$ \\
\hline $\mathrm{MP}$ & Z & iP & $27.3 \mathrm{~d}$ \\
\hline $\mathrm{U}$ & Z & iP & $27.5 \mathrm{~d}$ \\
\hline $\mathrm{Na}$ & Z & $\mathrm{eP}$ & $24.7 \mathrm{~d}$ \\
\hline $\mathrm{Pa}$ & Z & iP & $28.7 \mathrm{~d}$ \\
\hline $\mathrm{Hi}$ & Z & $\mathrm{eP}$ & $29.4 \mathrm{~d}$ \\
\hline $\mathrm{Ka}$ & Z & iP & $30.2 \mathrm{~d}$ \\
\hline $\mathrm{Ha}$ & Z & iP & $33.8 \mathrm{~d}$ \\
\hline U & PEZ & iP & $27.6 \mathrm{c}$ \\
\hline $\mathrm{U}$ & PEZ & iS & $11: 55: 09$ \\
\hline $\mathrm{U}$ & PEZ & iSS & $11: 58: 48$ \\
\hline $\mathrm{U}$ & PEZ & $i R$ & $12: 02: 41$ \\
\hline $\mathrm{U}$ & $\mathrm{PEE}$ & $i G$ & $12: 00: 21$ \\
\hline
\end{tabular}

C\&GS card 40-63:

$11: 38: 00.9$

$30.7^{\circ}$ S., $178.3^{\circ} \mathrm{W}$.

Kermadec Islands region $\mathrm{h}$ about $34 \mathrm{~km}$

Magnitude 6.75-7 (Pas), $6.5(\mathrm{Brk}), 6.2(\mathrm{CGS})$,

6.5 (HVO).

May 21

U PEZ eR 01:34:22

C\&GS card 45-63:

$00: 58: 07.4$

$56.0^{\circ}$ S., $123.9^{\circ} \mathrm{W}$.

South Pacific Ocean

$\mathrm{h}$ about $33 \mathrm{~km}$.

\section{May 21}

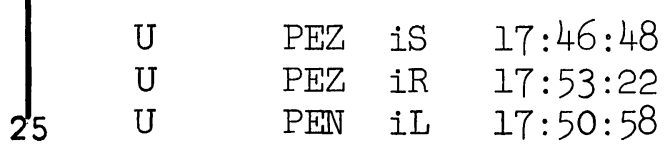


Table 5. --Distant earthquakes--Continued

May 21--Continued

C\&GS card 45-63:

$17: 30: 15.4$

11. $1^{\circ} \mathrm{S} ., 163.3^{\circ} \mathrm{E}$.

Solomon Is lands region

$\mathrm{h}$ about $33 \mathrm{~km}$

Magnitude 5.75-6 ( $\mathrm{Pal})$, 5.4 (CGS).

\section{May 22}

$\begin{array}{lllr}\text { M } & Z & \text { eP } & 14: 05: 31.6 \mathrm{c} \\ \mathrm{A} & \mathrm{Z} & \mathrm{eP} & 32.8 \mathrm{c} \\ \mathrm{MP} & \mathrm{Z} & \mathrm{eP} & 32.6 \mathrm{c} \\ \mathrm{U} & \mathrm{Z} & \mathrm{eP} & 32.1 \mathrm{c} \\ \mathrm{Ka} & \mathrm{Z} & \mathrm{eP} & 28.4 \mathrm{c} \\ \mathrm{Hi} & \mathrm{Z} & \text { iP } & 31.3 \mathrm{c} \\ \mathrm{Na} & \mathrm{Z} & \text { iP } & 35.3 \mathrm{c} \\ \mathrm{U} & \mathrm{PEE} & \text { iS } & 14: 12: 40 \\ \mathrm{U} & \mathrm{PEZ} & \text { iSS } & 14: 16: 04 \\ \mathrm{U} & \mathrm{PEZ} & \text { iR } & 14: 18: 56 \\ \mathrm{Ha} & \text { Z } & \text { Tmax } & 14: 56: 51\end{array}$

C\&GS card 39-63:

$13: 56: 43.0$

$48.6^{\circ} \mathrm{N} ., 154.7^{\circ} \mathrm{E}$.

Kurile Islands region

$\mathrm{h}$ about $22 \mathrm{~km}$

Magnitude 6.5 (Pas),

6 (Brk),

6.3 (CGS), 6.3 (HVO).

\section{May 22}

$\begin{array}{lllr}M & Z & \text { eP } & 15: 54: 33.6 \mathrm{c} \\ \mathrm{A} & \mathrm{Z} & \mathrm{eP} & 34.1 \mathrm{c} \\ \mathrm{N} & \mathrm{Z} & \mathrm{iP} & 33.9 \mathrm{c} \\ \mathrm{MP} & \mathrm{Z} & \mathrm{eP} & 34.3 \mathrm{c}\end{array}$

C\&GS card 42-63:

$15: 42: 48.6$

$4.3^{\circ}$ N., $127.9^{\circ}$ E.

Molucca Passage

$\mathrm{h}$ about $58 \mathrm{~km}$

Magnitude 5.0 (CGS).

\section{May 22}

M Z iP $16: 32: 15.1 \mathrm{c}$
May 22--Continued

C\&GS card 39-63:

$16: 25: 36.8$

$52.2^{\circ} \mathrm{N} ., 165.3^{\circ} \mathrm{W}$.

Fox Islands, Aleutian Islands

$\mathrm{h}$ about $33 \mathrm{~km}$

Magnitude 4.2 (CGS).

\section{May 22}

M Z iP 22:06:09.3 c

C\&GS card 41-63:

$21: 53: 02.5$

8. $2^{\circ} \mathrm{S} ., 115.7^{\circ} \mathrm{E}$.

Java Sea

$\mathrm{h}$ about $33 \mathrm{~km}$

Magnitude 5.6 (CGS).

\section{May 23}

M Z eP 01:03:40.7 d

C\&GS card 42-63:

$00: 51: 40.3$

$1.6^{\circ}$ N., $126.4^{\circ} \mathrm{E}$.

$\mathrm{h}$ about $33 \mathrm{~km}$

Molucca Passage

Magnitude 4.8 (CGS).

\section{May 23}

$\begin{array}{lllr}M & Z & \text { iP } & 03: 40: 30.6 \mathrm{c} \\ \mathrm{MP} & \mathrm{Z} & \mathrm{eP} & 29.9 \mathrm{c} \\ \mathrm{U} & \mathrm{Z} & \mathrm{eP} & 30.1 \mathrm{c} \\ \mathrm{Na} & \mathrm{Z} & \text { iP } & 27.9 \mathrm{~d} \\ \mathrm{H} i & \mathrm{Z} & \text { iP } & 31.6 \mathrm{~d} \\ \mathrm{Ka} & \mathrm{Z} & \mathrm{eP} & 32.1 \mathrm{~d} \\ \mathrm{Ha} & \text { Z } & \text { iP } & 35.3 \mathrm{~d}\end{array}$

C\&GS card 40-63:

$03: 33: 19.1$

$15.0^{\circ} \mathrm{S} ., 176.7^{\circ} \mathrm{W}$.

Fiji Islands region

$\mathrm{h}$ about $279 \mathrm{~km}$

Magnitude 5.4 (CGS).

\section{May 23}

$\begin{array}{lllll} & \mathrm{U} & \text { PEZ } & \text { iS } & 03: 52: 06 \\ 26 & \mathrm{U} & \text { PEZ } & \text { iR } & 03: 58: 32\end{array}$


Table 5.--Distant earthquakes--Continued

\section{May 23, 1963--Continued \\ C\&GS card 48-63: \\ $03: 35: 34.7$ \\ $10.9^{\circ} \mathrm{S} ., 163.3^{\circ} \mathrm{E}$. \\ Solomon Islands region \\ $\mathrm{h}$ about $33 \mathrm{~km}$ \\ Magnitude: 5.5 (CGS).}

\section{May 23}

$\begin{array}{lllr}M & Z & \text { iP } & 15: 23: 53.7 \mathrm{c} \\ \mathrm{A} & \mathrm{Z} & \mathrm{eP} & 54.0 \mathrm{c} \\ \mathrm{MP} & \mathrm{Z} & \text { iP } & 54.7 \mathrm{c} \\ \mathrm{U} & \mathrm{Z} & \mathrm{eP} & 53.9 \mathrm{c}\end{array}$

C\&GS card 43-63:

$15: 12: 05.7$

$6.0^{\circ} \mathrm{N} ., 126.1^{\circ} \mathrm{E}$.

Near east coast of Mindanao, Philippine Islands.

$\mathrm{h}$ about $88 \mathrm{~km}$

Magnitude 5.5 (CGS).

\section{May 26}

$\begin{array}{lllr}M & Z & \text { iP } & 23: 15: 38.4 \mathrm{c} \\ \mathrm{A} & \mathrm{Z} & \mathrm{eP} & 40.1 \mathrm{c} \\ \mathrm{N} & \mathrm{Z} & \text { iP } & 39.0 \mathrm{c} \\ \mathrm{Pa} & \mathrm{Z} & \mathrm{eP} & 40.0 \mathrm{c} \\ \mathrm{U} & \mathrm{PEZ} & \text { iR } & 23: 29: 15\end{array}$

C\&GS card 41-63:

$23: 06: 55.0$

$55.2^{\circ}$ N., $159.9^{\circ}$ E.

Near east coast of Kamchatka

$h$ about $47 \mathrm{~km}$

Magnitude 4.25 ( $\mathrm{PaI})$,

5.3 (CGS).

\section{May 27}

$\begin{array}{llll}M & Z & \text { iP } & 04: 07: 30.0 \mathrm{~d} \\ \mathrm{D} & \mathrm{Z} & \text { iP } & 30.7 \mathrm{c} \\ \mathrm{Pa} & \mathrm{Z} & \mathrm{eP} & 32.1 \mathrm{~d} \\ \mathrm{U} & \mathrm{PEE} & \text { iG } & 04: 19: 17 \\ \mathrm{U} & \mathrm{PEZ} & \text { iR } & 04: 21: 07\end{array}$

C\&GS card 42-63:

$03: 58: 47.9$

$55.3^{\circ}$ N., $160.1^{\circ} \mathrm{E}$.
May 27--Continued

C\&GS card--Continued

Near east coast of Kamchatka

$\mathrm{h}$ about $54 \mathrm{~km}$

Magnitude 5.25 (PaI), 5.7 (CGS).

May 29

$\begin{array}{lllr}\text { M } & \text { Z } & T \max & 19: 39: 44 \\ \text { A } & \text { Z } & T \max & 50 \\ \text { MP } & \text { Z } & T \max & 25 \\ \mathrm{~Pa} & \text { Z } & T \max & 20 \\ \mathrm{Na} & \text { Z } & T \max & 34\end{array}$

C\&GS card 46-63:

$18: 27: 19.1$

$22.6^{\circ} \mathrm{S} ., 114.4^{\circ} \mathrm{W}$.

Easter Island region

$\mathrm{h}$ about $33 \mathrm{~km}$

Magnitude 4.7 (CGS).

\section{May 29}

$\begin{array}{lllr}\text { M } & \text { Z } & T \operatorname{Tmax} & 19: 42: 57 \\ \text { A } & \text { Z } & T \max & 52 \\ \text { D } & \text { Z } & T \max & 51 \\ \text { MP } & \text { Z } & T \max & 44\end{array}$

C\&GS card 49-63:

$18: 30: 25$

$24.4^{\circ} \mathrm{S} ., 114.7^{\circ} \mathrm{W}$.

Easter Island region

$\mathrm{h}$ about $33 \mathrm{~km}$

Magnitude 4.5 (CGS).

\section{May 30}

U PEZ iR 07:37:56

C\&GS card 46-63:

$06: 56: 09.3$

$54.2^{\circ}$ S., $143.7^{\circ} \mathrm{E}$.

South of Australia

$\mathrm{h}$ about $33 \mathrm{~km}$

Magnitude 5.25-5.5 (Pal). 
Table 5.--Distant earthquakes--Continued

$\frac{\text { June 2, } 1963}{\text { U PEZ eR 22:05:29 }}$
C\&GS card 46-63:
21:04:24.2
58.5. S. 15.6 W.
Sandwich Islands region
h about 50 km
Magnitude 6-6.25 (Pal).
5.9 (CGS).

June 3

$\begin{array}{llll}M & \text { Z } & \text { eP } & 07: 45: 56.6 \mathrm{c} \\ \mathrm{U} & \text { PEZ } & \text { eR } & 08: 03: 01\end{array}$

C\&GS card 44-63:

$07: 35: 54.3$

$34.2^{\circ}$ N., $138.7^{\circ} \mathrm{E}$.

Honshu, Japan

$h$ about $43 \mathrm{~km}$

Magnitude 5.3 (CGS).

\section{June 4}

$\begin{array}{lllr}M & Z & \text { iP } & 21: 16: 45.5 \mathrm{~d} \\ \mathrm{~A} & \mathrm{Z} & \mathrm{eP} & 45.6 \mathrm{~d} \\ \mathrm{MP} & \mathrm{Z} & \text { iP } & 45.8 \mathrm{~d} \\ \mathrm{U} & \mathrm{Z} & \mathrm{eP} & 45.7 \mathrm{c} \\ \mathrm{Hi} & \mathrm{Z} & \mathrm{eP} & 46.3 \mathrm{c} \\ \mathrm{U} & \mathrm{PEN} & \mathrm{eL} & 21: 37: 02 \\ \mathrm{U} & \mathrm{PEZ} & \mathrm{eR} & 21: 41: 10\end{array}$

C\&GS card 46-63:

$21: 04: 42.3$

1. $2^{\circ}$ S., $127.3^{\circ} \mathrm{E}$.

Halmahera region

$h$ about $31 \mathrm{~km}$

Magnitude 5.2 (CGS).

\section{June 6}

$\begin{array}{rrrr}M & Z & \text { iP } & 05: 30: 56.9 \mathrm{~d} \\ \mathrm{~A} & \mathrm{Z} & \mathrm{eP} & 56.0 \mathrm{~d} \\ \mathrm{D} & \mathrm{Z} & \mathrm{eP} & 56.1 \mathrm{~d}\end{array}$

C\&GS card 46-63:

$05: 18: 55.1$

$19.9^{\circ}$ N., $120.2^{\circ} \mathrm{E}$.

Off north coast of Luzon,

Philippine Islands.

$\mathrm{h}$ about $33 \mathrm{~km}$
June 6--Continued

C\&GS card--Continued

Magnitude 5.8 (CGS).

\section{June 7}

U PEZ IS 19:47:15

U PEZ eR 19:53:53

C\&GS card 49-63:

$19: 30: 35.6$

8. $5^{\circ}$ N., $103.1^{\circ} \mathrm{W}$.

Clipperton Island region

$\mathrm{h}$ about $33 \mathrm{~km}$

Magnitude 5.5-5.75 (Brk), $5.25-5.5(\mathrm{Pal})$,

4.9 (CGS).

June 7

U PEE II 22:53:31

C\&GS card 51-63:

$2.2: 37: 30.0$

$15.3^{\circ}$ S., $173.2^{\circ} \mathrm{W}$.

Samoa Islands region

$\mathrm{h}$ about $33 \mathrm{~km}$

Magnitude 5.0 (CGS).

June 10

$M \quad Z \quad$ iP $04: 29: 34.4$ a

A Z iP $\quad 32.4 \mathrm{~d}$

$\begin{array}{lll}\mathrm{D} & \mathrm{E} & 32.3 \mathrm{c}\end{array}$

$\begin{array}{llll}\mathrm{U} & \mathrm{Z} & \mathrm{P} & 33.2 \mathrm{~d}\end{array}$

U PEE eG 04:53:58

$\mathrm{U} \quad \mathrm{PEZ}$ iR 04:58:14

C\&GS card 47-63:

$04: 16: 37.7$

$55.4^{\circ} \mathrm{S} ., 146.4^{\circ} \mathrm{E}$.

$800 \mathrm{~km}$ west of Macquarie Islands $\mathrm{h}$ about $33 \mathrm{~km}$

Magnitude 6.25 (Pas), 5.75-6 (Pal), 6.1 (CGS), 6.7 (HVO). 
Table 5.--Distant earthquakes--Continued

\begin{tabular}{llll}
\hline \multicolumn{3}{c}{ June } & 10, 1963 \\
M & Z & iP & $06: 52: 06.5 \mathrm{~d}$ \\
$\mathrm{~A}$ & $\mathrm{Z}$ & iP & $04.9 \mathrm{~d}$ \\
$\mathrm{U}$ & $\mathrm{Z}$ & eP & $05.3 \mathrm{~d}$ \\
$\mathrm{U}$ & PEN & eSS & $07: 08: 54$ \\
U & PEN & eG & $07: 16: 32$ \\
U & PEZ & iR & $07: 20: 21$
\end{tabular}

C\&GS card 48-63:

$06: 39: 04.0$

55. $3^{\circ} \mathrm{S} ., 146.1^{\circ} \mathrm{E}$.

$800 \mathrm{~km}$ west of Macquarie Islands $\mathrm{h}$ about $18 \mathrm{~km}$

Magnitude 6.25-6.5 (Pas),

$$
\begin{aligned}
& 6.25-6.5 \text { ( } \mathrm{Pal}), \\
& 6.0 \text { (CGS), } \\
& 6.6 \text { (HVO). }
\end{aligned}
$$

\section{June 16}

$\begin{array}{llll}\text { M } & \text { Z } & T \max & 10: 05: 34 \\ \text { A } & \text { Z } & T \max & 10: 06: 02 \\ \text { D } & \text { Z } & T \max & 10: 05: 58 \\ \text { MP } & \text { Z } & T \max & 10: 05: 48 \\ \text { U } & \text { Z } & T \max & 10: 05: 55 \\ \mathrm{~Pa} & \mathrm{Z} & T \max & 10: 05: 51 \\ \mathrm{Hi} & \mathrm{Z} & T \max & 10: 05: 23 \\ \mathrm{Ha} & \mathrm{Z} & T \max & 10: 04: 58\end{array}$

C\&GS card 49-63:

$09: 19: 54.8$

$50.8^{\circ}$ N., $129.5^{\circ} \mathrm{W}$.

Vancouver Island region

$\mathrm{h}$ about $33 \mathrm{~km}$

Magnitude 4.4 (CGS).

\section{June 17}

$\begin{array}{lllr}\text { M } & \text { Z } & \text { iP } & 18: 40: 05.7 \mathrm{c} \\ \text { D } & \text { Z } & \text { iP } & 06.8 \mathrm{c} \\ \text { MP } & \text { Z } & \text { eP } & 08.2 \mathrm{c} \\ \text { U } & \text { Z } & \text { eP } & 06.2 \mathrm{c} \\ \text { Hi } & \text { Z } & \text { eP } & 04.5 \mathrm{c} \\ \text { Ha } & \text { Z } & \text { iP } & 18: 39: 58.7 \mathrm{c} \\ \text { U } & \text { PEE } & \text { eL } & 18: 49: 57 \\ \text { U } & \text { PEZ } & \text { eR } & 18: 51: 53\end{array}$

C\&GS card 49-63:

$18: 32: 14.5$

$60.4^{\circ}$ N., $140.8^{\circ} \mathrm{W}$.
June 17--Continued

C\&GS card--Continued

Southwestern Yukon

$\mathrm{h}$ about $33 \mathrm{~km}$

Magnitude 5.25-5.5 ( $\mathrm{Pal})$

\begin{tabular}{|c|c|c|c|}
\hline M & Z & iP & $09: 20: 53.9 \mathrm{~d}$ \\
\hline A & Z & iP & $55.3 \mathrm{~d}$ \\
\hline $\mathrm{U}$ & Z & $\mathrm{eP}$ & 54.2 \\
\hline $\mathrm{U}$ & PEZ & $e R$ & $09: 44: 06$ \\
\hline
\end{tabular}

$$
5.1 \text { (CGS). }
$$

\section{June 19}

C\&GS card 49-63:

09:09:04.0

$4.7^{\circ}$ N., $126.5^{\circ}$ E.

Talaud Islands region

$\mathrm{h}$ about $83 \mathrm{~km}$

Magnitude 5.25-5.5 ( $\mathrm{Pal}$ ), 6.2 (CGS),

6. 0 (HVO).

June 19

$\begin{array}{rrrr}\mathrm{Ha} & Z & \text { iP } & 18: 31: 19.4 \mathrm{~d} \\ \mathrm{Na} & \mathrm{Z} & \text { iP } & 15.4 \mathrm{c} \\ \mathrm{Hi} & \mathrm{Z} & \text { eP } & 17.4 \mathrm{~d}\end{array}$

C\&GS card 49-63:

$18: 22: 09.6$

$3.5^{\circ} \mathrm{S} ., 153.4^{\circ} \mathrm{E}$.

New Ireland region

Felt: Rabaul

Magnitude 5.1 (CGS).

$\mathrm{h}$ about $279 \mathrm{~km}$. 
Table 5.--Distant earthquakes--Continued

\section{June 24, 1963}

\begin{tabular}{|c|c|c|c|}
\hline M & Z & iP & $04: 34: 12.0$ \\
\hline A & Z & iP & 13.5 \\
\hline D & Z & iP & 13.8 \\
\hline $\mathrm{MP}$ & Z & $\mathrm{eP}$ & 13.6 \\
\hline $\mathrm{U}$ & Z & iP & 13.1 \\
\hline $\mathrm{Pa}$ & Z & eP & 10.0 \\
\hline $\mathrm{Na}$ & Z & iP & 16.8 \\
\hline $\mathrm{Hi}$ & Z & iP & 11.1 \\
\hline $\mathrm{Ha}$ & Z & iP & 01.6 \\
\hline U & PEZ & iP & 13 \\
\hline U & PEZ & iPP & $04: 35: 41$ \\
\hline $\mathrm{U}$ & PEZ & iPPP & $04: 36: 25$ \\
\hline $\mathrm{U}$ & PEZ & $i R$ & $04: 44: 54$ \\
\hline тT & $\mathrm{PEE}$ & is & $04: 40: 20$ \\
\hline U & PEE & iL & $04: 43: 04$ \\
\hline $\mathrm{Ha}$ & Z & Tmax & $05: 12: 41$ \\
\hline
\end{tabular}

C\&GS card 46-63:

$04: 26: 37.9$

$59.5^{\circ}$ N., $151.7^{\circ} \mathrm{W}$.

Cook Inlet

$\mathrm{h}$ about $52 \mathrm{~km}$

Magnitude 6.75 (Pas),

5.7 (CGS),

6.5 (HVO).

\section{June 24}

$\begin{array}{llrr}\text { M } & \text { Z } & \text { Tmax } & 11: 04: 19 \\ \text { A } & \text { Z } & T \max & 10 \\ \text { D } & \text { Z } & T \max & 30 \\ \text { MP } & \text { Z } & T \max & 17 \\ \text { U } & \text { Z } & T \max & 12 \\ \mathrm{~Pa} & \text { Z } & T \max & 12 \\ \mathrm{Hi} & \mathrm{Z} & T \max & 11: 03: 53 \\ \mathrm{Ha} & \mathrm{Z} & T \max & 11: 03: 23\end{array}$

C\&GS card 52-63:

$10: 17: 02.5$

$52.8^{\circ}$ N. , $131.9^{\circ} \mathrm{W}$.

Queen Charlotte Islands region $\mathrm{h}$ about $38 \mathrm{~km}$

Magnitude 3.9 (CGS).
June 24

\begin{tabular}{|c|c|c|c|}
\hline M & Z & iP & $15: 08: 51.9 \mathrm{~d}$ \\
\hline A & Z & iP & $51.7 \mathrm{~d}$ \\
\hline $\mathrm{N}$ & $\mathrm{Z}$ & iP & 52.1 \\
\hline MP & Z & eP & 51.0 \\
\hline $\mathrm{Na}$ & Z & iP & 47.8 \\
\hline $\mathrm{Hi}$ & Z & iP & 53.9 \\
\hline $\mathrm{Ha}$ & Z & iP & 56 \\
\hline
\end{tabular}

C\&GS card 53-63:

$15: 01: 44.2$

$15.5^{\circ}$ S., $177.5^{\circ} \mathrm{W}$.

Fiji Islands region

$h$ about $412 \mathrm{~km}$

Magnitude 5.0 (CGS).

June 24

M Z iP $16: 24: 08.4 \mathrm{~d}$

$M \quad Z \quad i P P \quad 16: 25: 21.0 \mathrm{~d}$

A Z eP 16:24:08.7 d

$\begin{array}{llll}\text { U } & \text { Z } & \text { ePP } & 22.7 \mathrm{~d}\end{array}$

$U$ PEZ iS 16:29:39

U PEZ iR 16:33:13

Ha Z T Tmax 16:59:36

C\&GS card 49-63:

$16: 17: 15.4$

$52.3^{\circ}$ N., $171.2^{\circ} \mathrm{W}$.

Fox Islands region

Aleutian Islands

$h$ about $33 \mathrm{~km}$

Magnitude 5.4 (CGS), 5.5 (HVO).

June 25

Ha Z Tmax 09:06:41

C\&GS card 52-63:

$08: 26: 21.9$

$44.3^{\circ} \mathrm{N} ., 129.1^{\circ} \mathrm{W}$.

off coast of Oregon

$h$ about $31 \mathrm{~km}$

Magnitude 4.5 (CGS). 
Table 5.--Distant earthquakes--Continued

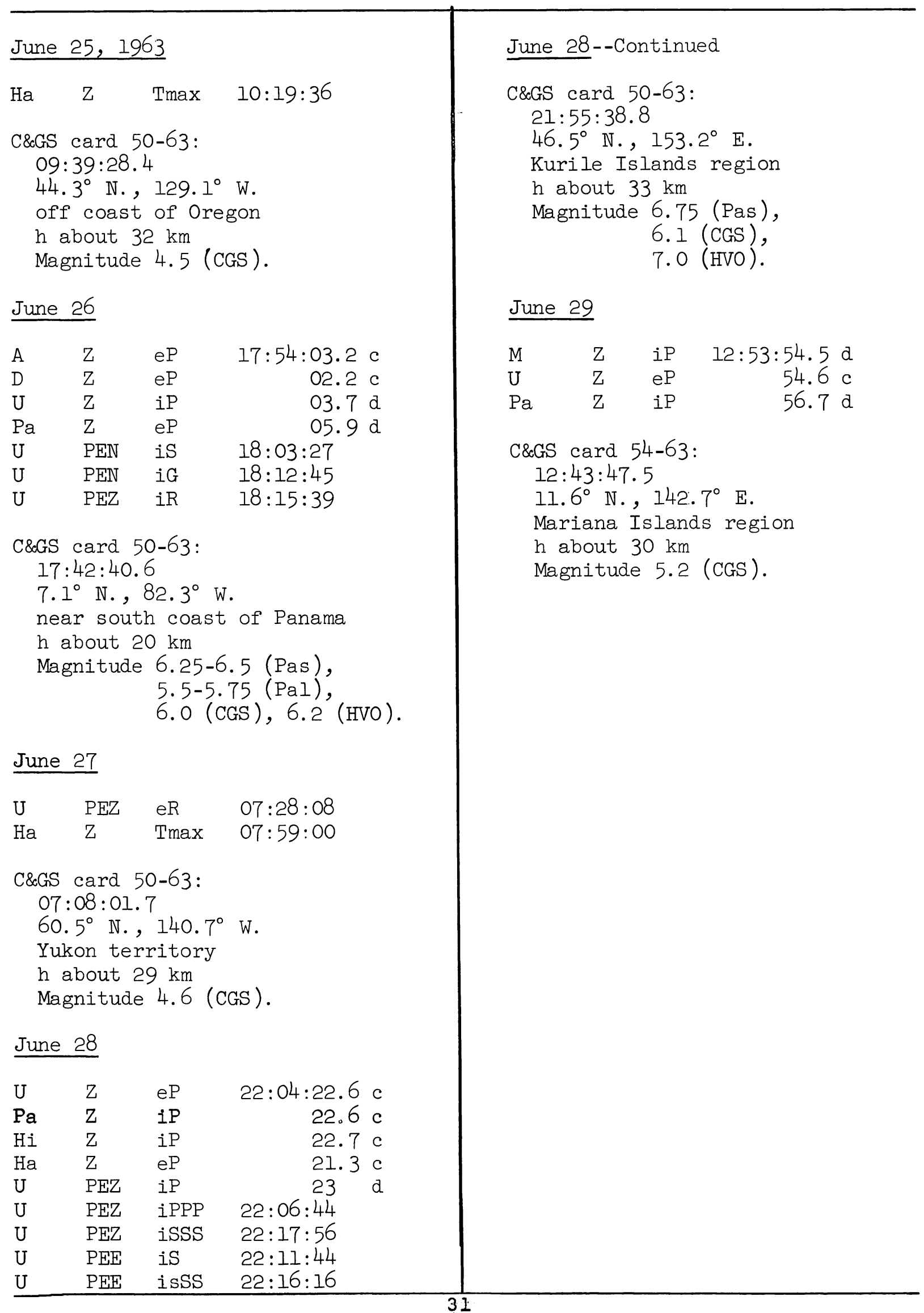


The following persons or agencies reported "felt" earthquakes during the second quarter, 1963. Their assistance is gratefully acknowledged.

North Hawaii

Mrs. Eklund

Mrs. Lindsey

Puuanahulu School

Miss Wallace

Kilauea summit region

Mrs. Hansen

Mrs. Mist

Mrs. Wentworth

Mrs. Cuskelly

KMC residents

Mr. Ryall

Mrs. Yong

Mrs. Hirano

Miss English

Kona coast

Mr. B. Berman

Mr. Ladd

Puna

Mr. Edwards

Central Hawaii

Kulani Honor Camp

Hilo region

Mrs. Shoemaker

Mr. S. Ho

Mr. Baldwin

Miss Patten

Mrs. Carter $\underline{\text { Kau region }}$

Mr. Mizuba

Kau Police station

Kahuku Ranch

Lt. C. Araujo

Mr. Godfrey

Kapapala Ranch residents

Mrs. Billings

Mrs. Walters

Mr. Meinecke

Mrs. Schattauer 


\section{UNITED STATES \\ DEPARTMEITT OF THE INTERIOR \\ GEOLOGICAL SURVEY}

\section{HAWAIIAN VOLCANO OBSERVATORY}

SUMMARY 31

July, August, and September, 1963

By

Robert Y. Koyanagi, Arnold T. Okamura,

Willie T. Kinoshita, J. G. Moore, and Howard A. Powers

\section{Issued December 1964}

Observatory Staff

Geology

J. G. Moore (Scientist-in-charge)

D. I. Peck, geologist, arrived 7-5-63

C. K. Wentworth

Geochemistry:

Geophysics:

W. T. Kinoshita, geophysicist, arrived 6-17-63

G. Kojima

R. Y. Koyanagi

R. T. Okamura

Support:

E. T. Endo

J. G. Forbes

W. H. Francis

B. J. Loucks

H. L. Krivoy

A. T. Okamura

A. S. Ryall

N. Sherrill

A. Yamamoto

Visiting Japanese scientists

(Arrived 6-29-63 for 6 months' cooperative program)

T. Minakami (Group leader), D. Shimozuru, S. Aramaki, T. Miyazaki,

C. Kurihara, and S. Hiraga 
U.S. -Japan cooperative study of Kilauea Volcano-... I

Chronological summary-1.. 3

Tilting of the ground around Kilauea caldera-......... 8

Seismic summary-a 14

Persons or agencies reporting felt earthquakes during the quarter- 40

\section{Illustrations}

Figure A. Map of the Kilauea caldera region at the summit of

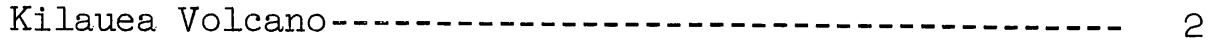

B. 1963 faults and cracks along the Koae fault system of Kilauea's south flank mapped by James G. Moore-..-.- 4

C. Hourly frequency plot of Kilauea seismic events

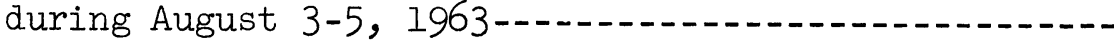

1. Map of the island of Hawaii showing seismograph stations operated by the Geological Survey and

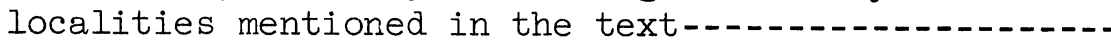

2a. Tilting of the ground around Kilauea caldera May 12

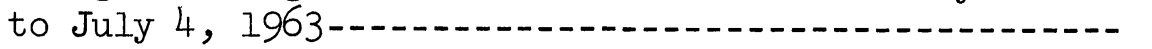

2b. Tilting of the ground around Kilauea caldera,

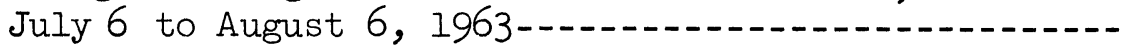

Tables

Table 1. Tilt coordinates at Uwekahuna Vault, July, August, and

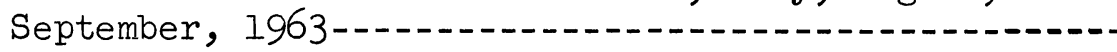

2a. Tilt coordinates and changes at bases around Kilauea

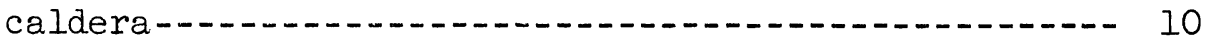

2b. Tilt coordinates and changes at bases around

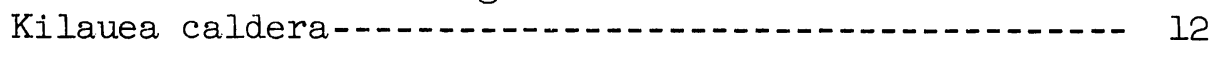

3. Numbers of earthquakes and minutes of tremor recorded on seismographs around Kilauea caldera-...............

4. Local earthquakes recorded by seismographs of the U.S. Geological Survey-a 20

5. Distant earthquakes 30 


\section{U.S. -Japan cooperative study of Kilauea Volcano}

For 6 months, beginning in the summer of 1963, a team of Japanese scientists lived and worked at Kilauea Volcano in close cooperation with the staff of the Volcano Observatory. This study was part of a program of scientific cooperation between the United States and Japan; the program originated in talks between President Kennedy and Prime Minister Ikeda in June 1961. The United States' participation is financed by the National Science Foundation and Japan's participation by the Japanese Ministry of Education.

The main group of Japanese scientists which arrived in Hawaii June 29, included Takeshi Minakami, Professor of Geophysics, University of Tokyo, leader; Daisuke Shimozuru, Assistant Professor of Geophysics, University of Kyushu; and Shigeo Aramaki, Assistant in Geology, University of Tokyo. Kosuke Kamo, Geophysicist, University of Kyoto, joined the team later. Several technicians accompanied the group.

The principal program of the Japanese scientists was the monitoring of seismic activity in the Kilauea summit area. To do this, a net of about 16 short-period seismometers (mainly vertical instruments) was placed in and near Kilauea caldera in the summit region of the volcano (fig. A). The instruments telerecorded at the Observatory. Six instruments recorded continuously on smoked paper drums and the others recorded a few hours per day (or longer during periods of exceptional activity) on photographic paper.

While the team was in Hawaii, seismic recordings were made of several noteworthy events: earthquakes and tremors associated with the eruptions of August and October; the collapses of July and August; several other large earthquakes and earthquake swarms; and explosions set off as part of the Geological Survey's program of refraction seismology.

The Observatory staff joined with the Japanese team in an offshore investigation of the south flank of Kilauea Volcano. The Kagoshima Maru, a 1,000-ton fisheries research vessel from Kagoshima University, which was on a training voyage in Hawaiian waters, was utilized in a 4-day echo-sounding program in August. The ship made about $400 \mathrm{~km}$ of depthprofiling traverses over a submarine part of Kilauea Volcano directly south of the summit region. Observatory members sailed on the ship and aided in the operation of the echo-sounder, and members of the Japanese team joined the Observatory staff in fixing the position of the ship from three transit stations on land.

The first data-gathering stage of the U.S. -Japan cooperative program was completed in early 1964 when the Japanese team left Hawaii. The second stage--analysis of the data--will continue for 2 years. Results will be compared and data integrated at future meetings, terminating in the fall of 1965. 


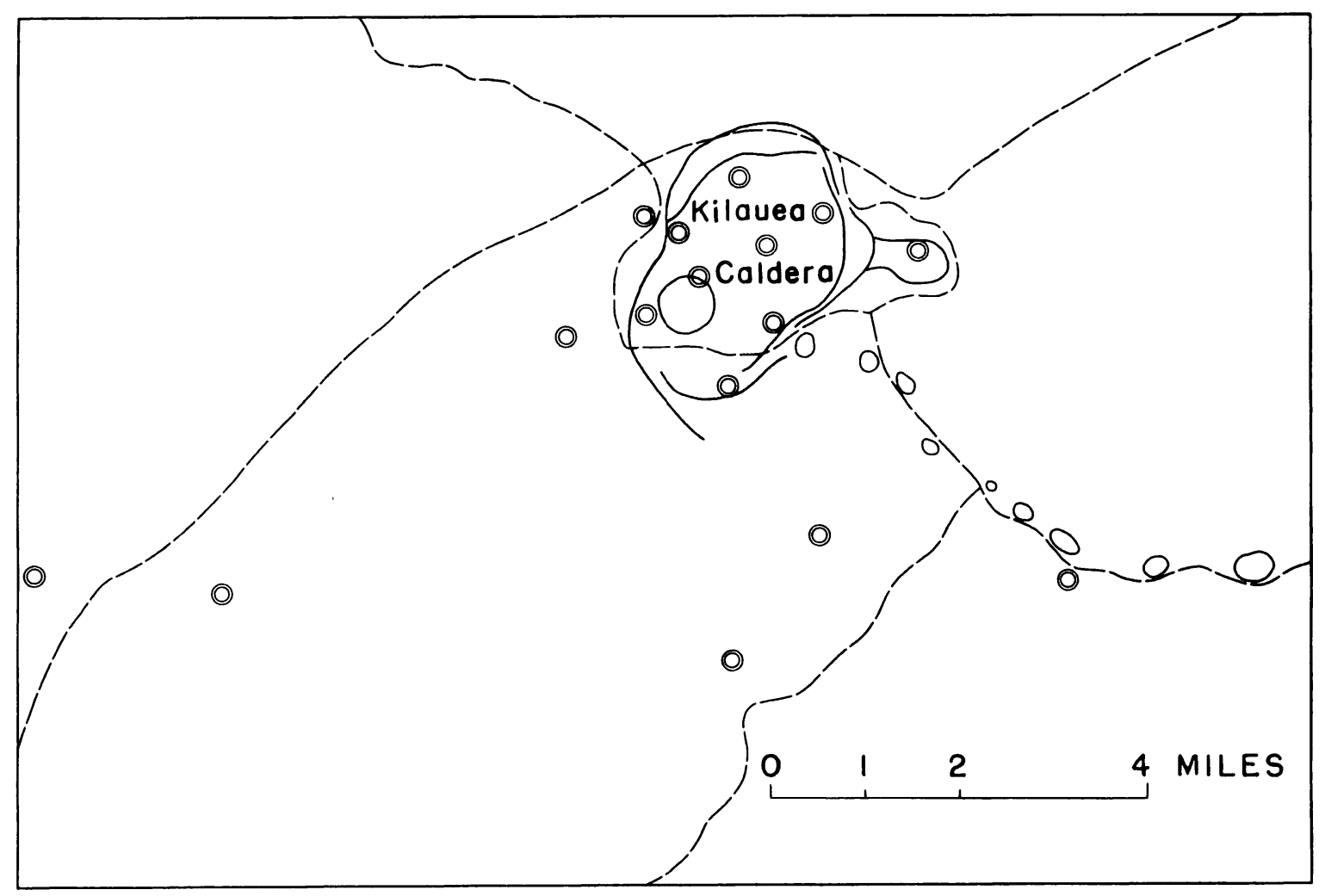

Figure A. --Map of the Kilauea caldera region at the summit of Kilauea Volcano. Locations of seismometers operated by visiting Japanese team are shown by double circles. 
The month preceding the opening of this quarter was exceedingly quiet in terms of any kind of seismic activity, and no clear trend of tilting was indicated by the short-base tiltmeter. However, during this quarter there were two episodes of summit collapse, two swarms of Kaoiki quakes, and two surface eruptions of lava (if we include the first week of October in the period of generalization).

A sharp spasm of seismic activity that began at 9:50 p.m. July 1 , 1963, included a great many felt earthquakes, a countless swarm of small quakes, and probably intermittent tremor. Rapid collapse tilting began at Uwekahuna shortly before midnight, and at about the same time cracks opened and pavement buckled in the road at Devil's Throat (fig. B). This structural crisis was over by July 5 and no lava had appeared at the surface. However, the Kilauea summit showed about the same amount of tilt collapse as it had shown during the December 1962 eruption along the upper Puna rift. Also upward swelling in part of this rift, as determined by precise leveling of the bench marks along the Chain of Craters road, was as much as 3 feet in the center of the disturbed zone. From these observations it is inferred that strong structural disturbance along the Puna rift and its junction with the Koae fault system permitted the movement of perhaps 10 million cubic yards of magma from under the summit region into the Puna rift system.

During the rest of July, tilting at Uwekahuna was moderately strong westerly, a direction associated with tumescence, but there was no noteworthy seismic activity.

On August 3, shallow tremor began at 9:25 p.m. and continued, accompanied by a swarm of shallow earthquakes centered near Aloi that began 15 minutes later. The quakes were felt only feebly in the Kilauea summit area 6 miles northwest, but their intensity caused concern at Ainahou Ranch 2 miles south of Aloi. The seismic crisis lasted only about 2 hours, but several new cracks intersected the Chain of Craters road, and leveling revealed surface subsidence of a 2 -mile section of the road, greatest (.4 ft) in the zone of cracking. A swarm of $30-\mathrm{km}$-deep quakes under the Kilauea summit followed the surface crisis by a few hours ( $f^{\prime} i g . C$ ).

There was comparative inactivity, both seismically and tiltwise, until August 21. On that day lava appeared, at about 6:00 p.m., from fissures across the floor and up the northeast wall of Alae Crater. The eruption lasted about 38 hours and formed a lake in the crater bottom containing almost a million cubic yards of tholeiitic basalt. Careful study of this lava lake was started immediately for the purpose of observing all aspects of the cooling history of the lava.

On August 26 at 8:49 a.m., an earthquake of magnitude 4.9 took place in the Kaoiki fault zone. The shock was felt strongly throughout the island of Hawaii. Aftershocks numbered about 50 per day for several weeks to follow. Two of the earliest of these were felt in the Kilauea summit area: one of magnitude 3.1 at 09:48, August 26, and one of magnitude 3.3 at $21: 35$, August 26. 


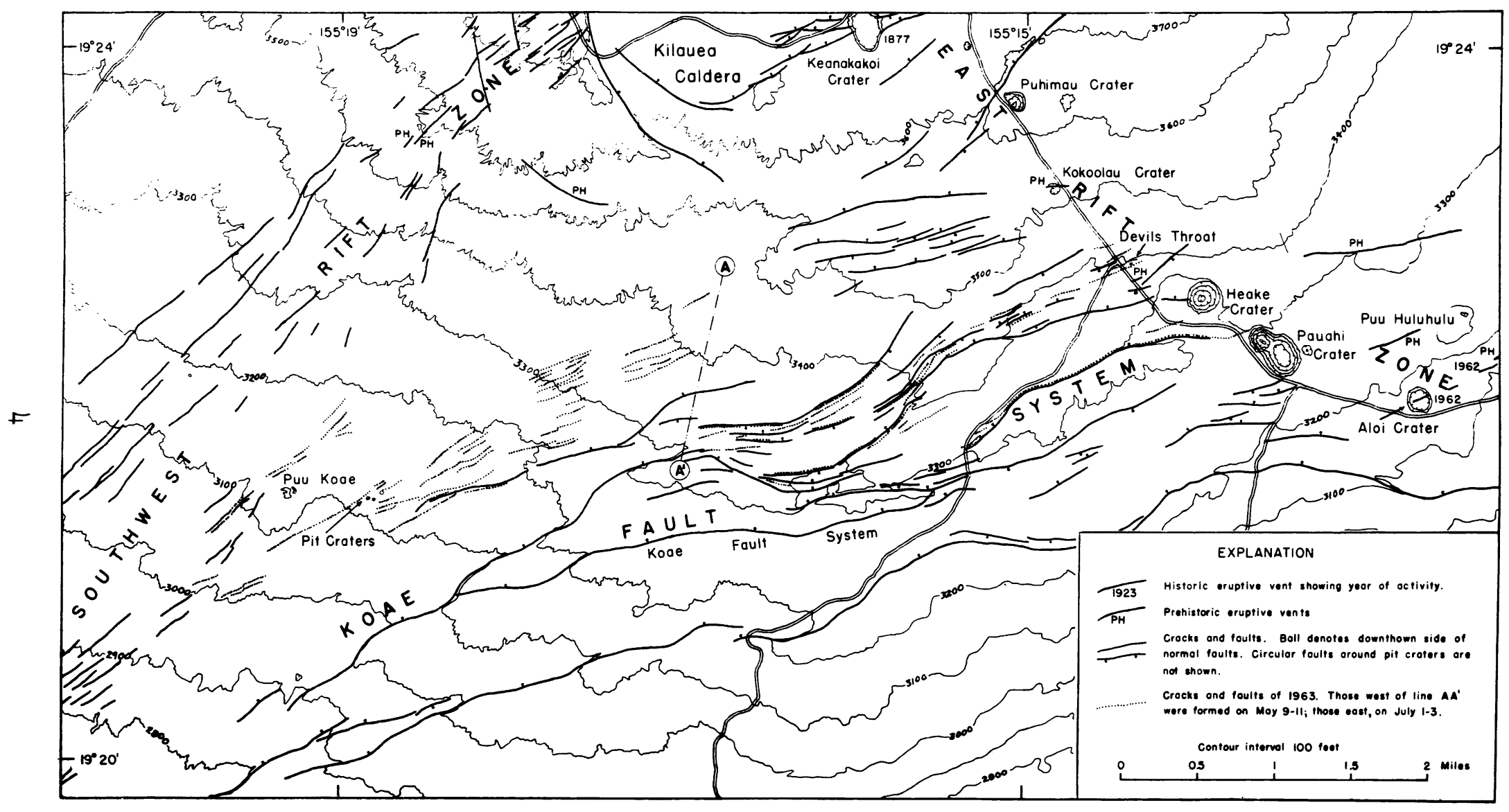

Figure B.--1963 faults and cracks along the Koae fault system on Kilauea's south flank mapped by James G. Moore (see also Summary 30). 


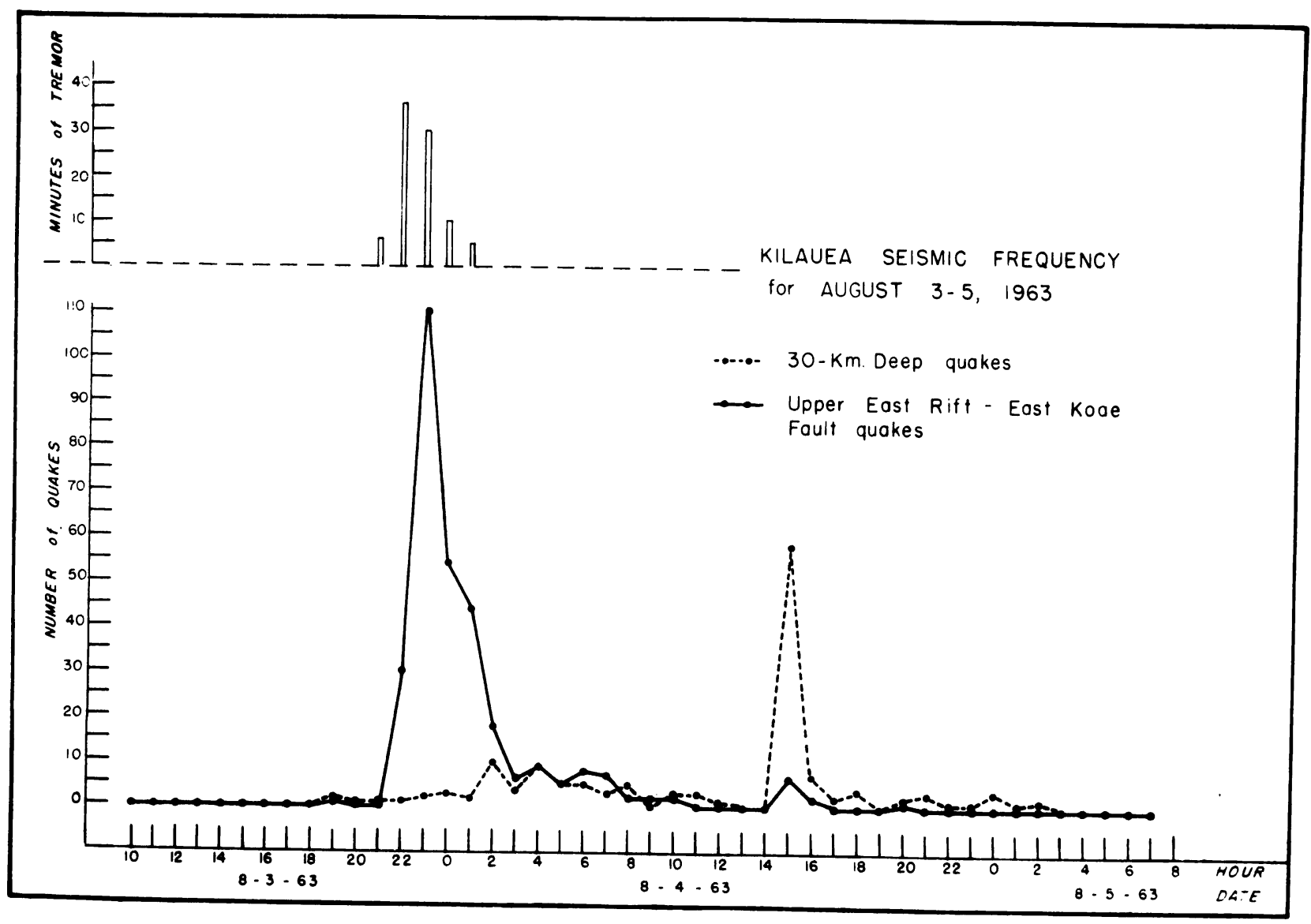

Figure C.--Hourly frequency plot of Kilauea seismic events during August 3-5, 1963. 
A second swarm of Kaoiki quakes was initiated by an earthquake of magnitude 4.8 that took place at 6:24 a.m. on September 2l. This quake also was felt throughout the island. Two of the many aftershocks were felt throughout the southern half of the island: one of magnitude 3.5 at 06:26, Sept.22, and a second of the same magnitude one minute later at 06:27.

Large numbers of small quakes centered under Kilauea summit accompanied the numerous aftershocks from the Kaoiki fault zone until the end of the quarter, in fact until the beginning of a flank eruption at Napau Crater on October 4, which will be reported in the next quarterly summary. 


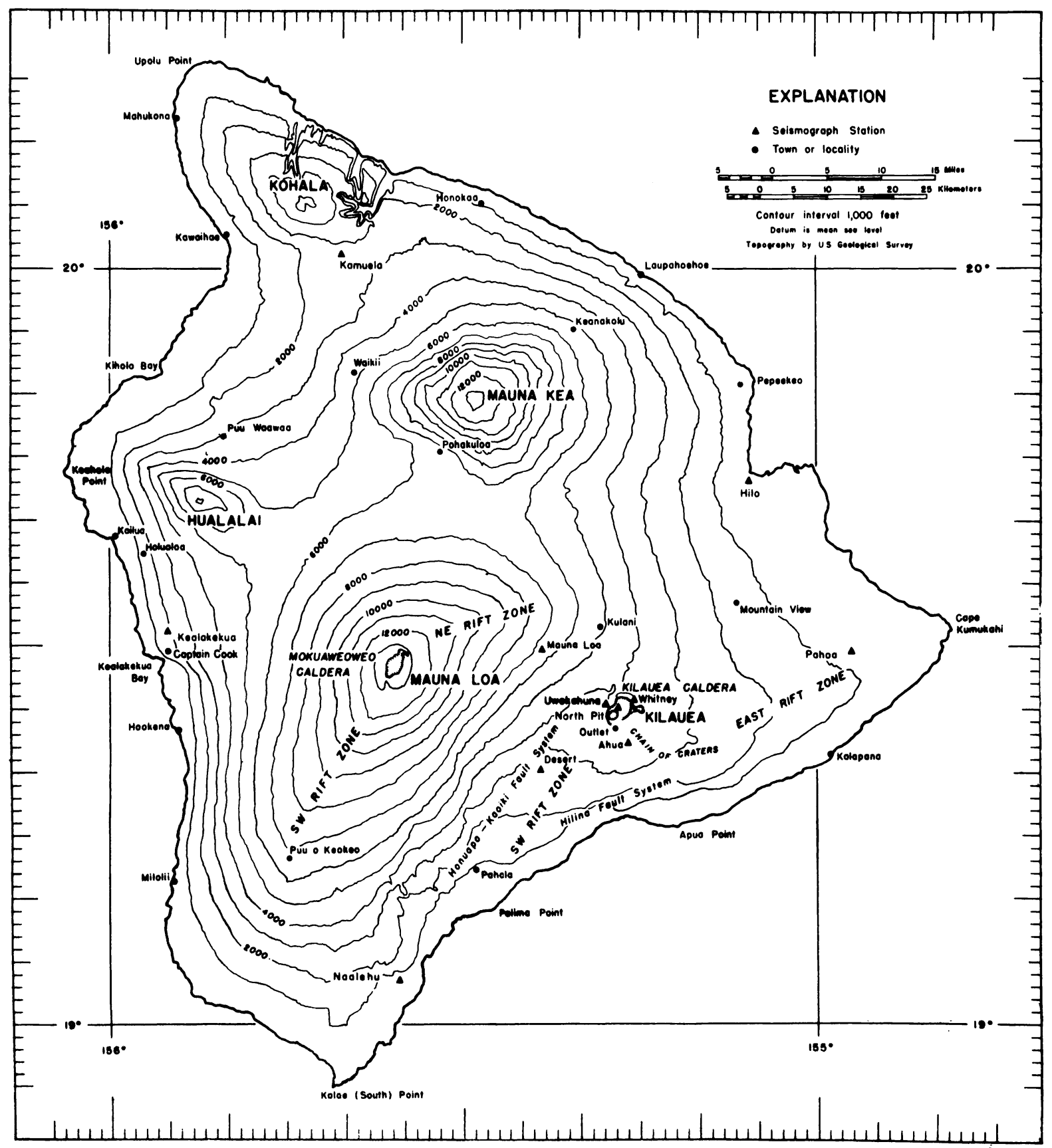

Figure 1.--Map of the island of Hawaii showing seismograph stations operated by the Geological Survey and localities mentioned in the text. Epicenters of local earthquakes are given in terms of geographic coordinates, which are indicated at the edges of the map. 
Tilting of the ground around Kilauea caldera.--Tilting of the ground around the summit of Kilauea is monitored daily by a short-base water-tube tiltmeter in Uwekahuna Vault, and at irregular intervals it is measured on a regional scale by means of a network of field tilt bases and a portable water-tube tiltmeter. The attitude of the ground surface at each tilt base is reported in terms of north-south and east-west tilt coordinates. Both coordinates at each station were arbitrarily set equal to 500 when measurements at that station were begun. Increasing tilt coordinates correspond to northward and eastward tilting of the earth's surface, i.e., to a relative subsidence toward the north and east. A one-unit change in coordinate corresponds to a tilting of 1 microradian ( $1 \mathrm{~mm}$ per $\mathrm{km}$ ) in the direction indicated. 
Table 1.--Tilt coordinates at Uwekahuna Vault, July, August, and September, 1963

\begin{tabular}{|c|c|c|c|c|c|}
\hline Date & $\mathrm{N}-\mathrm{S}$ & $E-W$ & Date & $\mathrm{N}-\mathrm{S}$ & $E-W$ \\
\hline June 30 & 505 & 497 & Sept. 1 & 503 & 491 \\
\hline July 7 & 498 & 504 & 8 & 504 & 494 \\
\hline 14 & 499 & 504 & 15 & 505 & 490 \\
\hline 21 & 502 & 499 & 22 & 505 & 482 \\
\hline 28 & 502 & 495 & 29 & 505 & 476 \\
\hline Aug. $\quad 4$ & 502 & 492 & & & \\
\hline 11 & 501 & 492 & & & \\
\hline 18 & 503 & 488 & & & \\
\hline 25 & 501 & 494 & & & \\
\hline
\end{tabular}


Table 2a. - Tilt coordinates and changes at bases around Kilauea caldera (see fig. 2a)

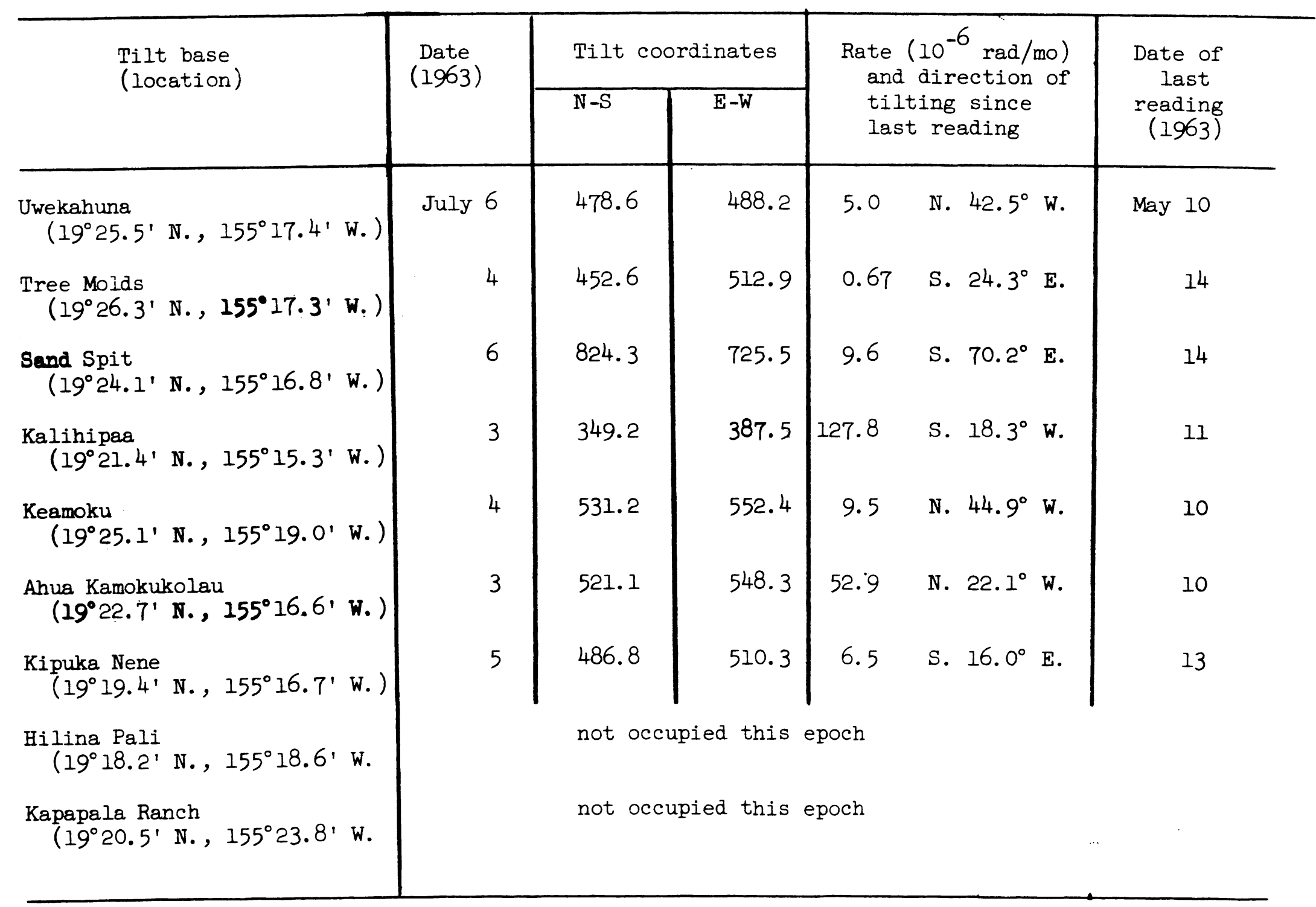




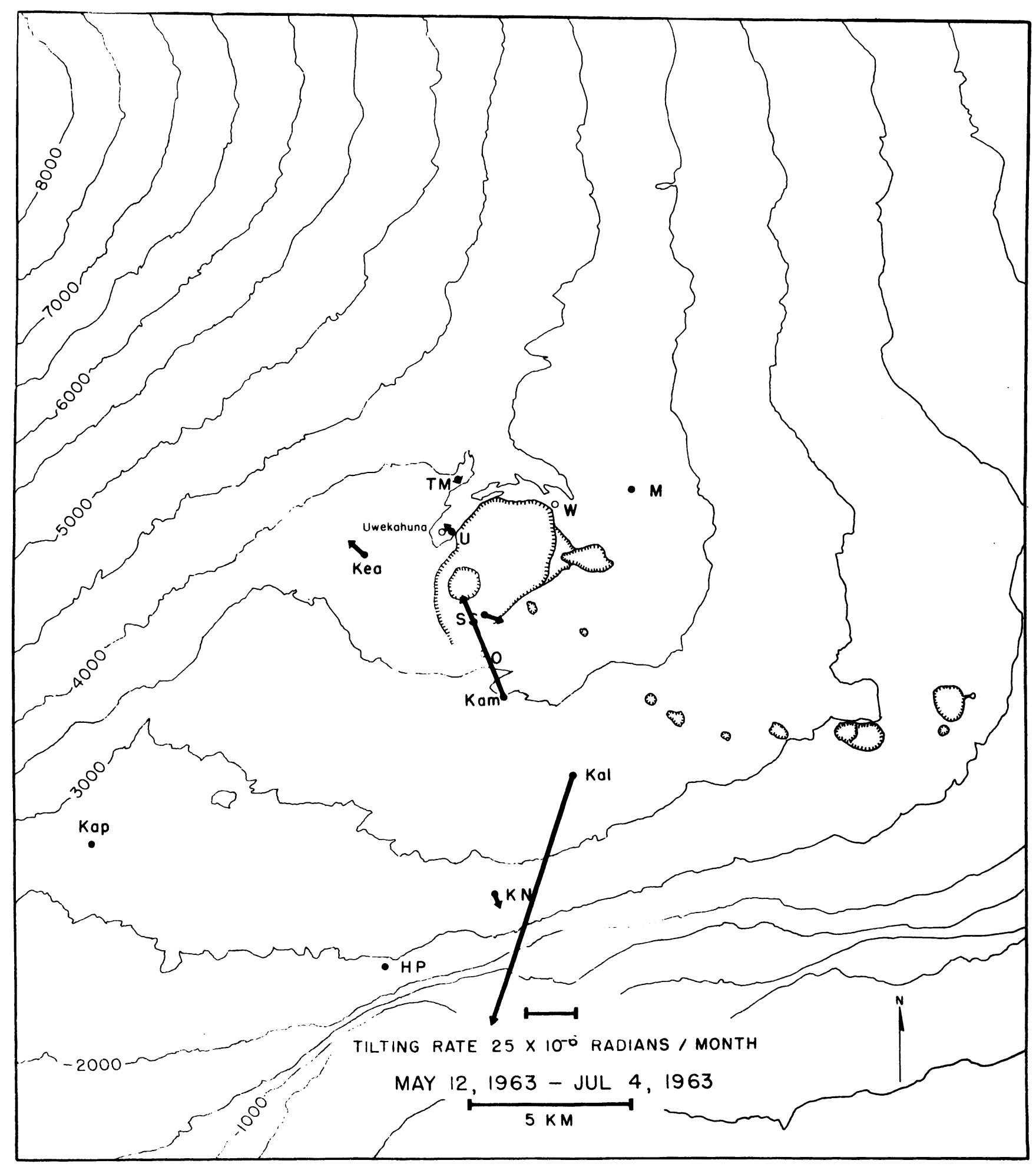

Figure 2a.--Tilting of the ground around Kilauea caldera, May 12 to July 4, 1963. The vector depicting tilting at a given tilt base points in the direction of maximum relatire subsidence and has a length proportional to the rate of tilting during the measurement interval. Closed circles represent field tilt bases; open circles, short-base water-tube tiltmeters. 
Table 2b. - Tilt coordinates and changes at bases around Kilauea caldera (see fig. 2b)

\begin{tabular}{|c|c|c|c|c|c|c|}
\hline \multirow{2}{*}{$\begin{array}{l}\text { Tilt Base } \\
\text { (location) }\end{array}$} & \multirow{2}{*}{$\begin{array}{l}\text { Date } \\
(1963)\end{array}$} & \multicolumn{2}{|c|}{ Tilt coordinates } & \multirow{2}{*}{\multicolumn{2}{|c|}{$\begin{array}{l}\text { Rate }\left(10^{-6} \mathrm{rad} / \mathrm{mo}\right) \\
\text { and direction of } \\
\text { tilting since } \\
\text { last reading }\end{array}$}} & \multirow{2}{*}{$\begin{array}{l}\text { Date of } \\
\text { : last } \\
\text { reading } \\
(1963)\end{array}$} \\
\hline & & $\mathrm{N}-\mathrm{S}$ & $\mathrm{E}-\mathrm{W}$ & & & \\
\hline $\begin{array}{l}\text { Uwekahuna } \\
\quad\left(19^{\circ} 25.5^{\prime} \text { N., } 155^{\circ} 17.4^{\prime} \text { W. }\right)\end{array}$ & Aug. 6 & 486.8 & 480.5 & 11.3 & N. $43^{\circ} \mathrm{W}$. & July 6 \\
\hline $\begin{array}{l}\text { Tree Molds } \\
\quad\left(19^{\circ} 26.3^{\prime} \text { N., } 155^{\circ} 17.3^{\prime} \text { W. }\right)\end{array}$ & 6 & 457.0 & 515.8 & 4.8 & N. $33^{\circ} \mathrm{W}$. & 4 \\
\hline $\begin{array}{l}\text { Sand Spit } \\
\quad\left(19^{\circ} 24.11^{\prime} \text { N., } 155^{\circ} 16.8^{\prime} \text { W. }\right)\end{array}$ & 12 & 821.4 & 727.0 & 2.6 & S. $26^{\circ} \mathrm{E}$. & 6 \\
\hline $\begin{array}{l}\text { Kalihipaa } \\
\quad\left(19^{\circ} 21.4^{\prime} \text { N., } 155^{\circ} 15.3^{\prime} \text { W. }\right)\end{array}$ & 5 & 336.1 & 377.8 & 14.8 & S. $37^{\circ} \mathrm{W}$. & 3 \\
\hline $\begin{array}{l}\text { Keamoku } \\
\quad\left(19^{\circ} 25.1^{\prime} N_{.}, 155^{\circ} 19.0^{\prime} \text { W. }\right)\end{array}$ & 12 & 536.9 & 544.7 & 6.8 & N. $54^{\circ} \mathrm{W}$. & 4 \\
\hline $\begin{array}{l}\text { Ahua Kamokukolau } \\
\quad\left(19^{\circ} 22.7^{\prime} \text { N. , } 155^{\circ} 16.6^{\prime} \text { W. }\right)\end{array}$ & 5 & 528.1 & 555.7 & 9.3 & N. $47^{\circ}$ E. & 3 \\
\hline $\begin{array}{l}\text { Kipuka Nene } \\
\quad\left(19^{\circ} 19.4^{\prime} \text { N., } 155^{\circ} 16.7^{\prime} \text { W. }\right)\end{array}$ & 9 & 484.8 & 510.1 & 1.7 & S. $06^{\circ} \mathrm{W}$ & 5 \\
\hline $\begin{array}{l}\text { Hilina Pali } \\
\quad\left(19^{\circ} 18.2^{\prime} \text { N., } 155^{\circ} 18.6^{\prime} \text { W. }\right)\end{array}$ & 8 & 497.5 & 500.7 & 1.4 & S. $17^{\circ} \mathrm{W}$ & May \\
\hline $\begin{array}{l}\text { Kapapala Ranch } \\
\quad\left(19^{\circ} 20.5^{\prime} \text { N., } 155^{\circ} 23.8^{\prime} \text { W. }\right)\end{array}$ & 8 & 496.9 & 503.5 & 0.3 & S. $24^{\circ}$ E. & 11 \\
\hline
\end{tabular}




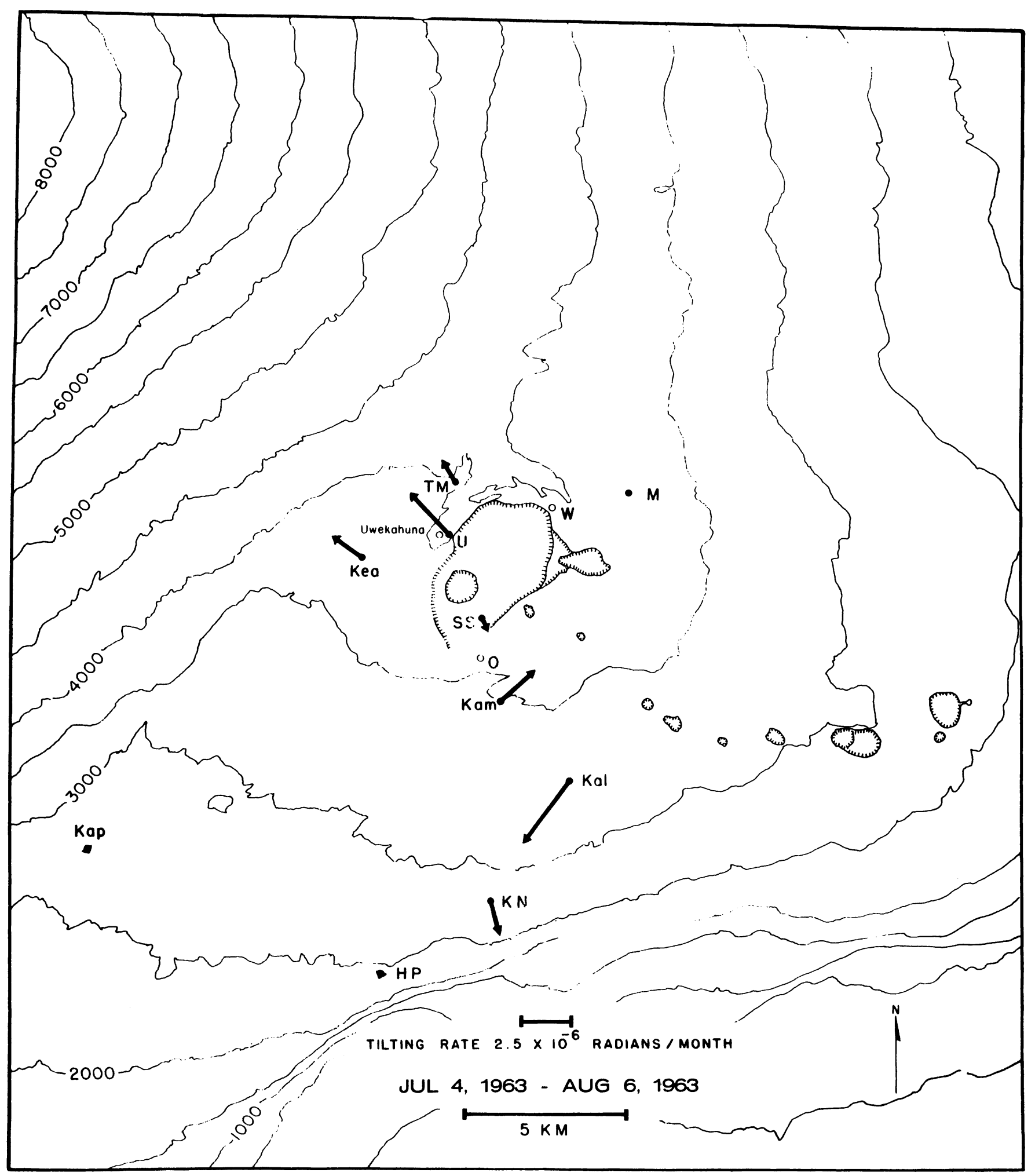

Figure 2b.--Tilting of the ground around Kilauea caldera, July 4 to August 6 , 1963. The vector depicting tilting at a given tilt base points in the direction of maximum relative subsidence and has a length proportional to the rate of tilting during the measurement interval. Closed circles represent field tilt bases; open circles, short-base water-tube tiltmeters. 
Seismic summary.--Events recorded by the U.S. Geological Survey seismograph network in Hawaii fall into two categories: local earthquakes and tremor originating in the region of the Hawaiian Islands, usually within $100 \mathrm{~km}$ of at least one seismograph, and distant earthquakes originating more than $3,000 \mathrm{~km}$ from Hawaii. As an index of seismic activity at Hawaiian volcanoes, daily counts of earthquakes and minutes of tremor recorded by seismographs in Hawaii are listed in table 3. The earthquakes are separated into groups on the basis of region of origin as determined by analysis of records obtained daily at the Observatory (U, M, A, D, N, MP). Earthquakes of magnitude 2.5 or greater are generally sufficiently well recorded to be located with greater precision: they are listed individually in table 4. Data on identifiable phases from distant earthquakes are listed in table 5.

Locations of the seismograph stations are shown on figure 1, and essential data on the stations were given in Summaries 25 and 30. 
Table 3. - Numbers of earthquakes and minutes of tremor recorded on seismographs

$$
\mathrm{U}, \mathrm{M}, \mathrm{A}, \mathrm{D}, \mathrm{N} \text {, and } \mathbb{M} \text { around Kilauea caldera }
$$

Tremor is separated into three categories: deep, intermediate, and shallow, on the basis of relative amplitudes on seismographs in the summit region. Unless otherwise stated, tremor is presumed to be associated with movement of magma within the central complex of Kilauea.

Earthquake categories are: Halemaumau rock slides, which are detected by the characteristic record they produce on the North Pit seismograph; shallow earthquakes in the Kilauea caldera region; shallow earthquakes along the SW. rift zone of Kilauea and the adjacent portion of the Kaoiki fault system: earthquakes along the eastern half of Kilauea's east rift zone--detected largely on the Pahoa shortperiod vertical; earthquakes from a source about $30 \mathrm{~km}$ beneath Halemaumau; earthquakes from the upper east rift zone and the adjacent fault systems of Kilauea's south flank (these are usually first arrivals at Ahua or Makaopuhi); and earthquakes from other regions: Kona, Mauna Kea, etc.

"Kalapana Trail" quakes formerly listed as column 9, have essentially ceased and are thus no longer listed.

A question mark (?) in the column indicates questionable interpretation of the records due to high concentration of seismic activity or an instrumental problem.

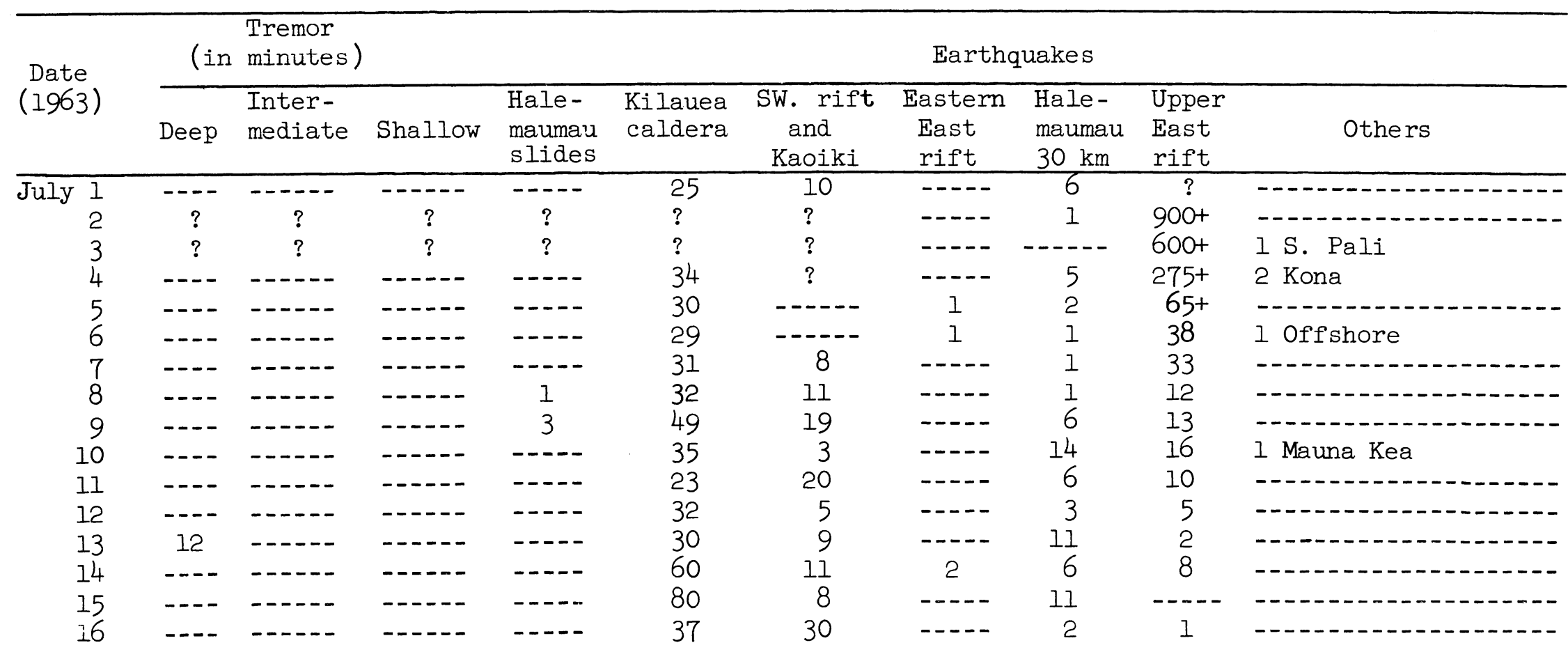


Table 3. - -Numbers of earthquakes and minutes of tremor recorded on seismographs

$\mathrm{U}, \mathrm{M}, \mathrm{A}, \mathrm{D}, \mathrm{N}$, and $\mathrm{EE}$ around Kilauea caldera--Continued

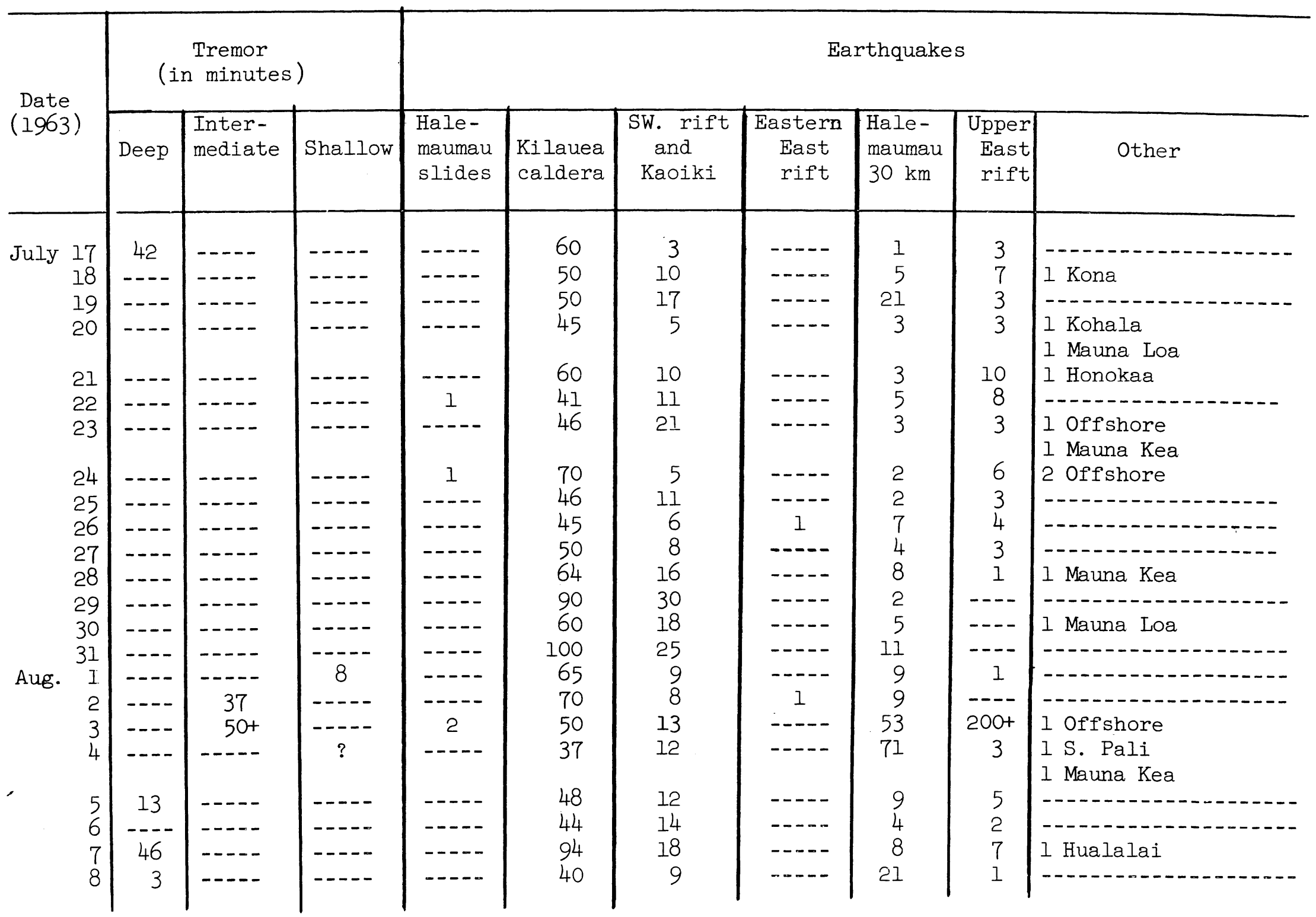


Table 3. - Numbers of earthquakes and minutes of tremor recorded on seismographs

$\mathrm{U}, \mathrm{M}, \mathrm{A}, \mathrm{D}, \mathrm{N}$, and $\mathrm{EE}$ around Kilauea caldera--Continued

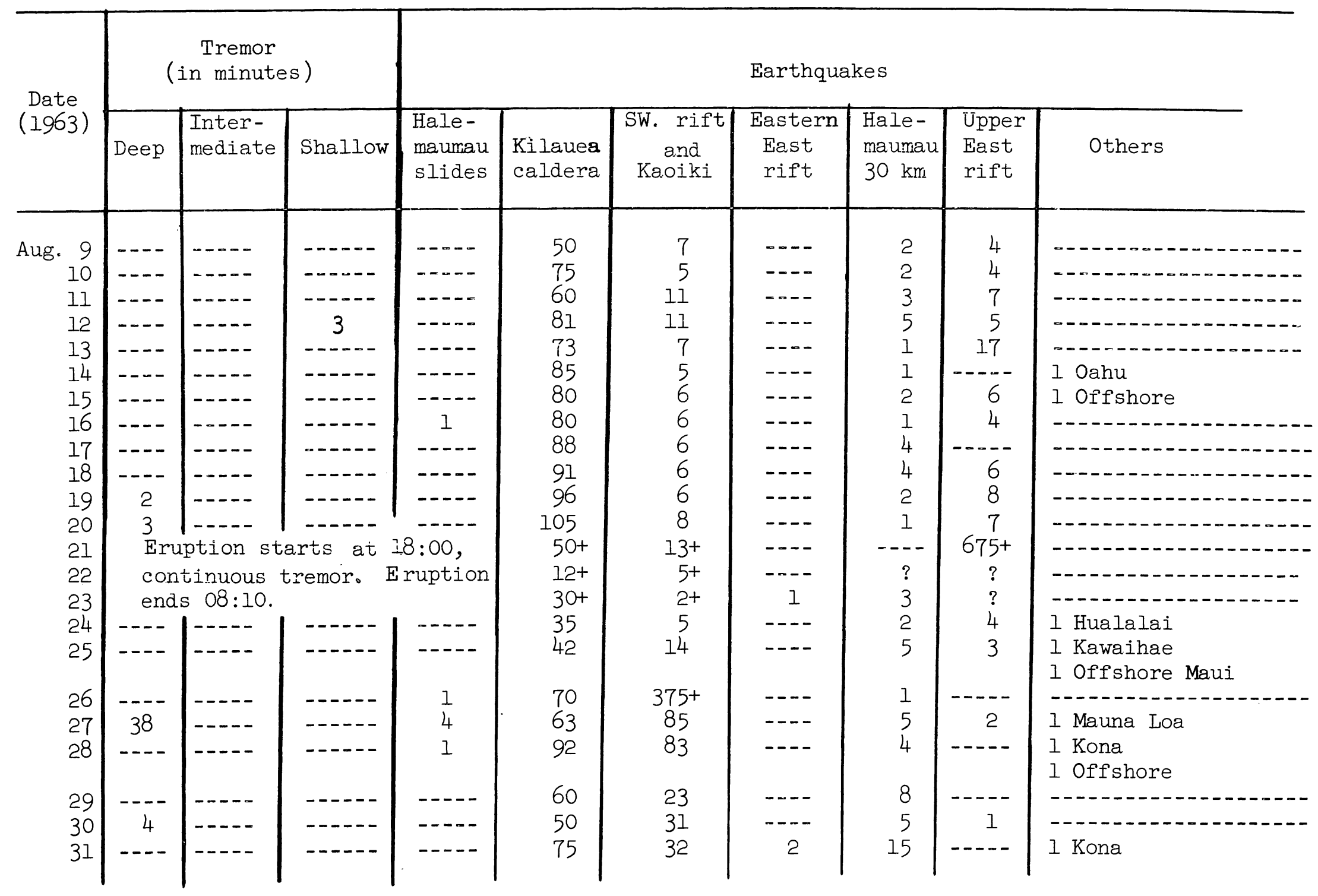


Table 3. - Numbers of earthquakes and minutes of tremor recorded on seismographs

$\mathrm{U}, \mathrm{M}, \mathrm{A}, \mathrm{D}, \mathrm{N}$, and $\mathrm{EE}$ around Kilauea caldera--Continued

\begin{tabular}{|c|c|c|c|c|c|c|c|c|c|c|}
\hline \multirow{2}{*}{$\begin{array}{l}\text { Date } \\
(1963)\end{array}$} & \multicolumn{3}{|c|}{$\begin{array}{c}\text { Tremor } \\
\text { (in minutes) }\end{array}$} & \multicolumn{7}{|c|}{ Earthquakes } \\
\hline & Deep & $\begin{array}{l}\text { Inter- } \\
\text { mediate }\end{array}$ & Shallow & $\begin{array}{l}\text { Hale- } \\
\text { maumau } \\
\text { slides }\end{array}$ & $\begin{array}{l}\text { Kilauea } \\
\text { caldera }\end{array}$ & $\begin{array}{l}\text { SW. rift } \\
\text { and } \\
\text { Kaoiki }\end{array}$ & $\begin{array}{c}\text { Eastern } \\
\text { East } \\
\text { rift }\end{array}$ & $\begin{array}{l}\text { Hale- } \\
\text { maumau } \\
30 \mathrm{~km}\end{array}$ & $\begin{array}{l}\text { Upper } \\
\text { East } \\
\text { rift }\end{array}$ & Others \\
\hline $\begin{aligned} & \text { Sept. } \\
& \\
& 2 \\
& 3 \\
& 4 \\
& 5 \\
& 6 \\
& \\
& 7 \\
& 8\end{aligned}$ & $\begin{array}{c}14 \\
\\
\cdots\end{array}$ & 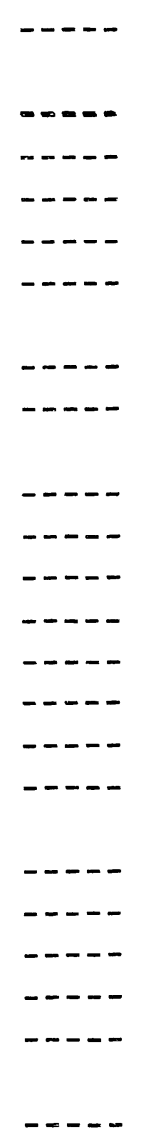 & 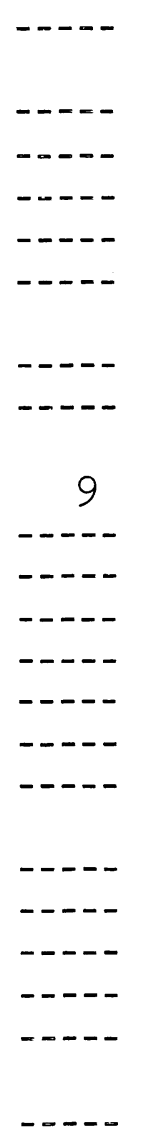 & 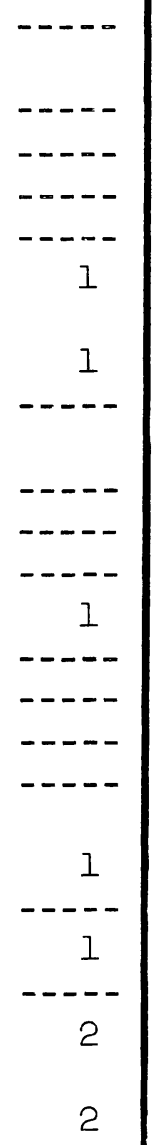 & $\begin{array}{r}70 \\
80 \\
102 \\
53 \\
65 \\
50 \\
60 \\
60 \\
\\
69 \\
120 \\
71 \\
60 \\
85 \\
110 \\
75 \\
120 \\
150 \\
115 \\
170 \\
85+ \\
155+ \\
120\end{array}$ & $\begin{array}{r}35 \\
35 \\
77 \\
20 \\
30 \\
30 \\
\\
30 \\
42 \\
\\
35 \\
24 \\
22 \\
24 \\
8 \\
28 \\
27 \\
32 \\
33 \\
44 \\
48 \\
75 \\
1960 \\
1300\end{array}$ & 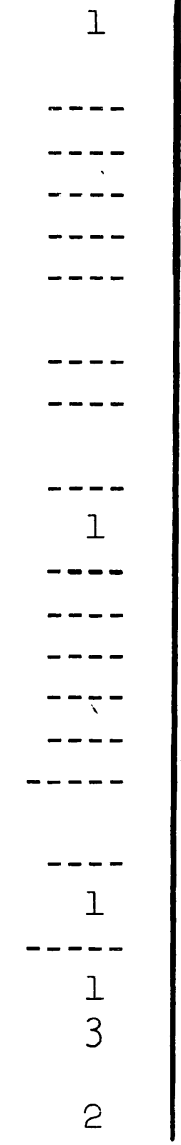 & $\begin{array}{r}9 \\
5 \\
3 \\
9 \\
2 \\
2 \\
\\
7 \\
1 \\
\\
7 \\
19 \\
4 \\
2 \\
1 \\
2 \\
7 \\
4 \\
7 \\
5 \\
7 \\
2 \\
-1- \\
1\end{array}$ & $\begin{array}{c}1 \\
8 \\
2 \\
-1 \\
1 \\
2 \\
2 \\
3 \\
2 \\
--- \\
-- \\
5 \\
5 \\
1 \\
2 \\
2 \\
6 \\
4 \\
5 \\
--- \\
2 \\
2\end{array}$ & 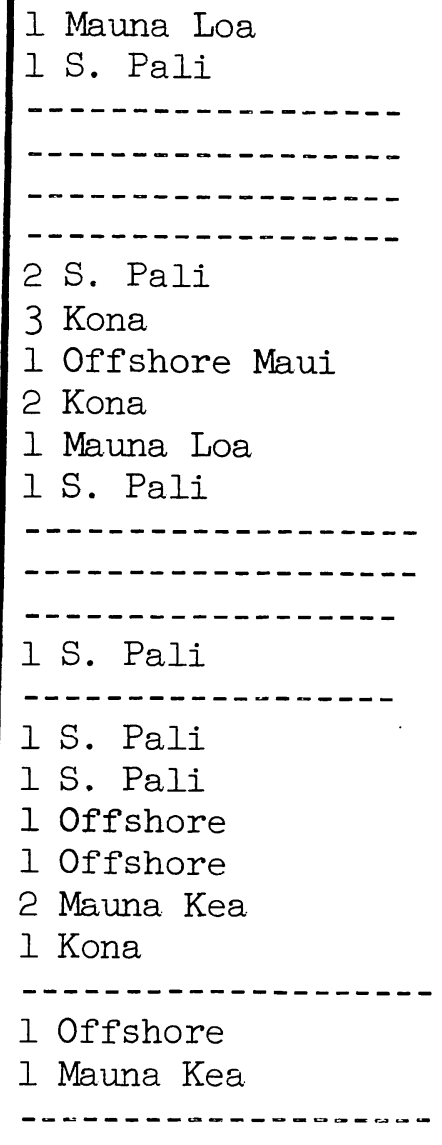 \\
\hline
\end{tabular}


Table 3.--Numbers of earthquakes and minutes of tremor recorded on seismographs

$$
\mathrm{U}, \mathrm{M}, \mathrm{A}, \mathrm{D}, \mathrm{N} \text {, and } \mathrm{EE} \text { around Kilauea caldera --Continued }
$$

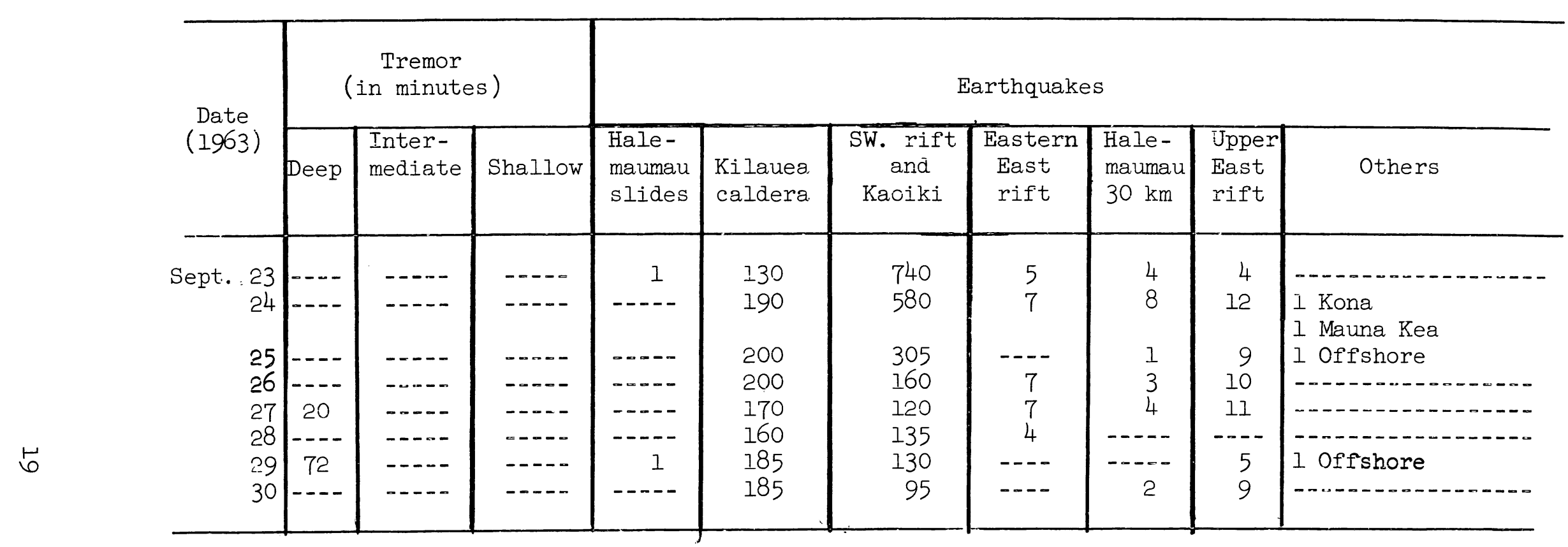


Table 4. --Local earthquakes recorded by seismographs of the U.S. Geological Survey July, August, and September, 1963

Entries for a given quake are: date, origin time (Hawaiian Standard Time), epicenter, depth, magnitude and felt report. Except for smaller earthquakes of special interest, only earthquakes with magnitudes of 2.5 or greater are included in the list.

In the following list, some origin times are followed only by "KM 30 " and a statement of magnitude. These are all members of a continuing family of quakes noted also in other Summaries. The best mean focus for this group is beneath Halemaumau at a depth of 30 kilometers (19 $24^{\circ} .1^{\prime} \mathrm{N} ., 1^{\circ} 17.1^{\prime}$ W. ).

In the following list a number of quakes are described as "Upper east rift" (see Summary 28). Further statistical study of this group which occurs in the swarm periods during July 1 to 6 and August 3 to 4 gives a mean epicenter $19^{\circ} 21.5^{\prime}$ N., $155^{\circ} 14^{\prime}$ W., about $2 \mathrm{~km}$ south of Aloi Crater at near-surface depth.

In Summary 24, "Kaoiki" was introduced as a symbol for listing any of a family of quakes with mean focus $19^{\circ} 24^{\prime} \mathrm{N} ., 155^{\circ} 25^{\prime} \mathrm{W} ., \mathrm{h}=3$ to $8 \mathrm{~km}$. This symbol is used in the following list.

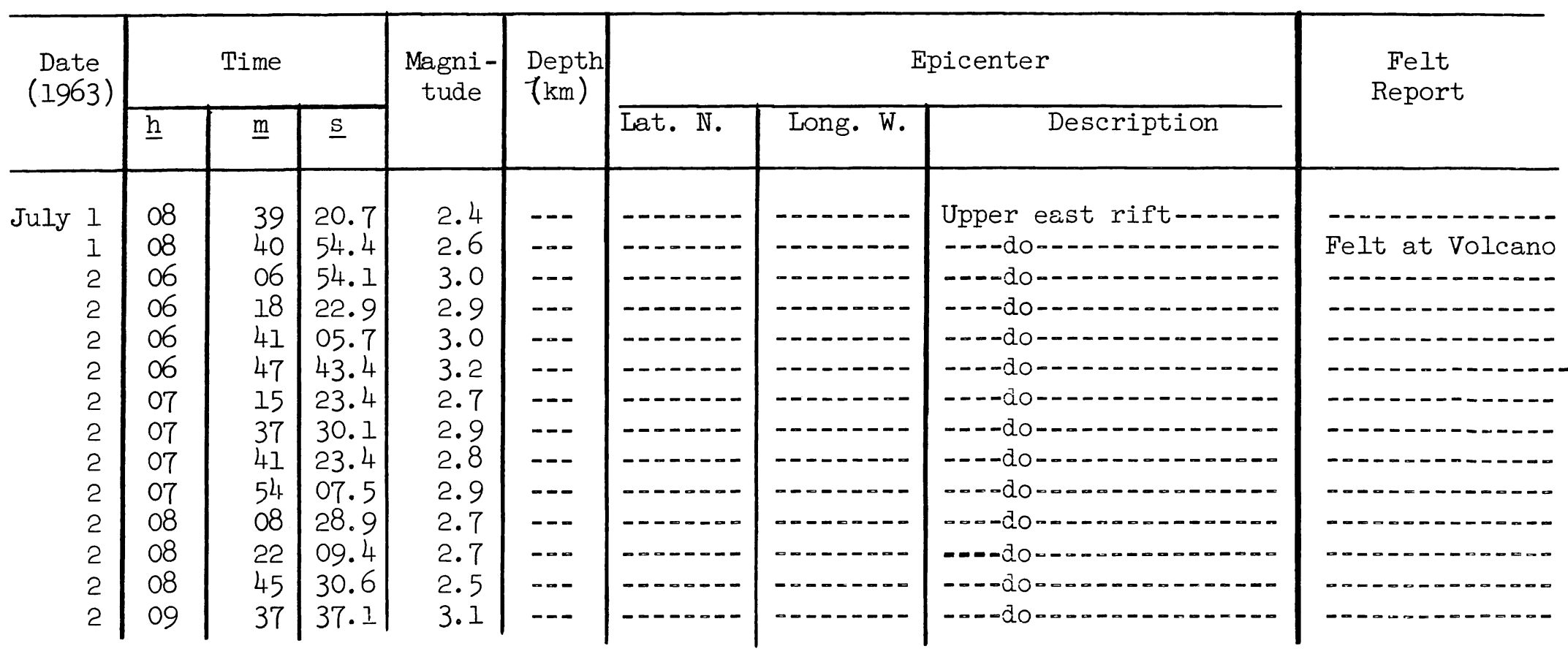


Table 4. --Local earthquakes recorded by seismographs of the U.S. Geological Survey, July, August, and September, 1963--Continued

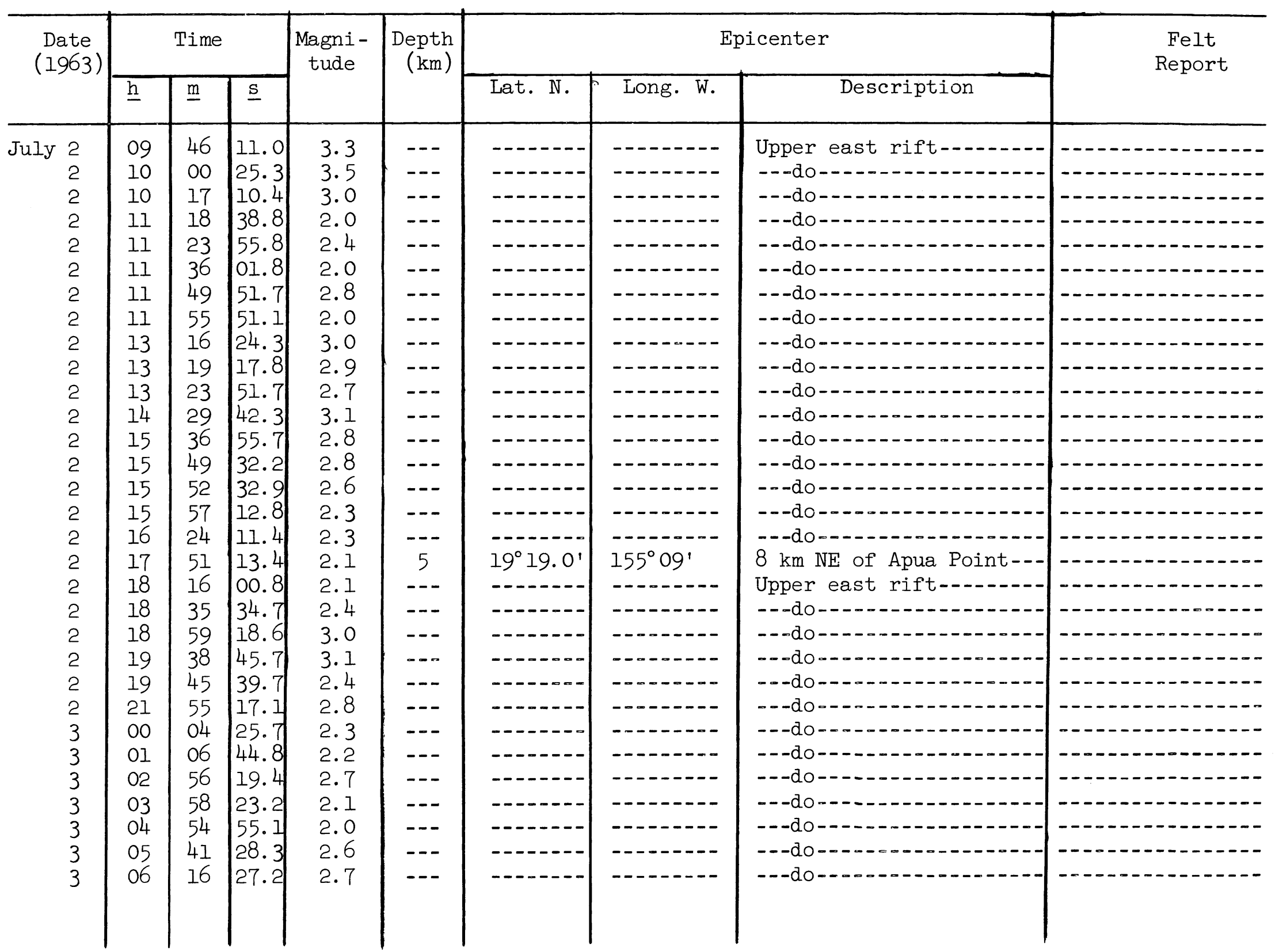


Table 4. --Local earthquakes recorded by seismographs of the U.S. Geological Survey, July, August, and September, 1963--Continued

\begin{tabular}{|c|c|c|c|c|c|c|c|c|c|}
\hline \multirow{2}{*}{$\begin{array}{l}\text { Date } \\
(1963)\end{array}$} & \multicolumn{3}{|c|}{ Time } & \multirow{2}{*}{$\begin{array}{l}\text { Magni- } \\
\text { tude }\end{array}$} & \multirow{2}{*}{$\begin{array}{c}\text { Depth } \\
(\mathrm{km})\end{array}$} & \multicolumn{3}{|r|}{ Epicenter } & \multirow{2}{*}{$\begin{array}{l}\text { Felt } \\
\text { Report }\end{array}$} \\
\hline & $\underline{\mathrm{h}}$ & $\underline{\mathrm{m}}$ & $\underline{\mathrm{s}}$ & & & Lat. N. & Long. W. & Description & \\
\hline July 3 & 06 & 30 & 15.7 & 3.1 & --- & ------- & -------- & Upper east rift-n-..- & Felt at Volcano \\
\hline 3 & 06 & 37 & 31.7 & 2.0 & --- & ------- & -------- & --- do --------------- & $-\div--------------$ \\
\hline 3 & 10 & 11 & 36.4 & 2.3 & --- & ------- & -------- & --- do ---------------- & --------------- \\
\hline 3 & 16 & 01 & 08.4 & 2.7 & --- & -------- & -------- & $---d \mathrm{do}-------------------$ & $----------\cdots---$ \\
\hline 3 & 16 & 28 & 53.4 & 2.0 & --- & ------- & -------- & $---\mathrm{d} 0--------------\cdots-\cdots$ & --------------- \\
\hline 3 & 19 & 30 & 34.8 & 2.6 & $1--$ & ------ & -------- & $---d o----\infty-------------$ & $---\infty------------$ \\
\hline 3 & 20 & 31 & 51.5 & 2.5 & --- & ------- & -------- & 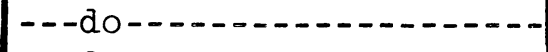 & 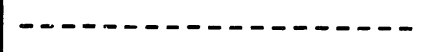 \\
\hline 4 & 00 & 12 & 50.9 & 3.3 & --- & ------ & -------- & $---d o-n-n-\cdots-n$ & Felt at Volcano \\
\hline 4 & 05 & 03 & 40.4 & 2.3 & --- & -------- & $-\infty-------$ & 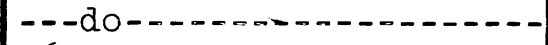 & $-----\infty---------$ \\
\hline 4 & 06 & 45 & 18.0 & 2.8 & 13 & $19^{\circ} 22.3^{\prime}$ & $155^{\circ} 55.6^{\prime}$ & $16 \mathrm{~km}$ SSW of Kealakekua & $-----\infty---------$ \\
\hline 4 & 07 & 38 & 31.4 & 2.9 & 13 & $19^{\circ} 25.3^{\prime}$ & $155^{\circ} 59.0^{\prime}$ & $13 \mathrm{~km} \mathrm{SW}$ of Kealakekua--- & $-\cdots-------------n$ \\
\hline 4 & 08 & 04 & 11.1 & 2.9 & --- & $-\cdots---n$ & -------- & Upper east rift $\ldots-\ldots$ & Felt at Volcano \\
\hline 4 & 08 & 39 & 09.9 & 2.6 & -- & $---\infty-\infty$ & -------- & 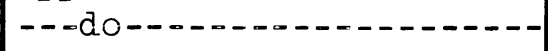 & $-\infty-------------$ \\
\hline 4 & 10 & 52 & 12.7 & 2.4 & --- & ------ & -------- & 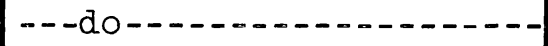 & ----------------0 \\
\hline 4 & 12 & 05 & 04.4 & 2.8 & $-\infty-$ & ------- & --------- & --- do ------------------- & 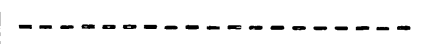 \\
\hline 4 & 17 & 01 & 02.7 & 2.6 & --- & $----\infty--$ & -------- & --- do $--\infty---\infty----------$ & $----------------n$ \\
\hline 4 & 17 & 55 & 57.4 & 2.8 & --- & & & $---\mathrm{do}-----------$ & $---------------n$ \\
\hline 4 & 17 & 58 & 13.9 & 2.5 & --- & $-----=--$ & & $---d \mathrm{~d} O------------$ & 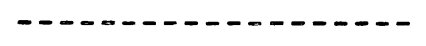 \\
\hline 4 & 20 & 06 & 26.7 & 2.0 & --- & $------n$ & $---\cdots---$ & --- do $---------\infty-\infty-----$ & $----------n-----$ \\
\hline 4 & 20 & 07 & 13.0 & 3.0 & --- & ------ & -------- & --- do $------n------n----$ & Felt at Volcano \\
\hline 5 & 03 & 19 & 27.2 & 2.5 & --- & ------ & ------- & $\mathrm{KM} 3 \mathrm{O}-\cdots-\cdots$ & $-----------0------$ \\
\hline 5 & 15 & 14 & 34.5 & 2.5 & --- & -------- & --------- & Upper east rift-n---n- & $-----------\infty-n$ \\
\hline 6 & 16 & 34 & 43.2 & 2.2 & --- & -------- & --------- & --- do $------\cdots-----n-\cdots---$ & $-\infty-\infty-\infty--\infty-\infty-\infty-\infty$ \\
\hline 6 & 17 & 12 & 01.1 & 2.4 & 3 & $19^{\circ} 16.8^{\prime}$ & $155^{\circ} 04.0^{\prime}$ & $13 \mathrm{~km} \mathrm{SW}$ of Kalapana--.-- & $---------\infty---\infty--$ \\
\hline 7 & 01 & 42 & 19.0 & 2.8 & --- & $---n--$ & -------- & $\mathrm{KM} 3 \mathrm{O}-\ldots \ldots$ & Felt at Volcano \\
\hline 10 & 16 & 32 & 44.0 & 3.4 & 13 & $19^{\circ} 55.5^{\prime}$ & $155^{\circ} 28.8^{\prime}$ & $18 \mathrm{~km}$ SSW of Honokaa-_-.- & Felt at Kamuela \\
\hline 11 & 01 & 39 & 26.0 & 3.3 & 8 & $19^{\circ} 16.5^{\prime}$ & $155^{\circ} 16.2^{\prime}$ & $\begin{array}{l}12 \mathrm{~km} \mathrm{~S} \text { of Ahua } \\
\text { seismometer. }\end{array}$ & Felt at Volcano \\
\hline 11 & 02 & 43 & $57 \cdot 5$ & 3.8 & 3 & $19^{\circ} 15.2^{\prime}$ & $155^{\circ} 10.1^{\prime}$ & $3 \mathrm{~km}$ ESE of Apua Point--- & $\begin{array}{l}\text { Felt at Volcano } \\
\text { to Hilo. }\end{array}$ \\
\hline 11 & 03 & 10 & 14.7 & 3.5 & --- & ------ & $-\infty----$ & Upper east rift & $\begin{array}{l}\text { Felt at Volcano to } \\
\text { Hilo. }\end{array}$ \\
\hline 11 & 11 & 29 & 19.8 & 2.5 & -- & $----\infty-$ & ------- & IIM 3O-n. & $------\infty----\infty----n$ \\
\hline 12 & 21 & 36 & 11.5 & 2.2 & --- & ------ & ------- & Upper east rift $\ldots-\cdots$ & $=-----------------$ \\
\hline
\end{tabular}


Table 4.--Local earthquakes recorded by seismographs of the U.S. Geological Survey, Ji:ly, August, and September, 1963--Continued

\begin{tabular}{|c|c|c|c|c|c|c|c|c|c|c|}
\hline \multirow{2}{*}{\multicolumn{2}{|c|}{$\begin{array}{l}\text { Date } \\
\text { (1963) }\end{array}$}} & \multicolumn{3}{|c|}{ Time } & \multirow{2}{*}{$\begin{array}{l}\text { Magni- } \\
\text { tude }\end{array}$} & \multirow{2}{*}{$\begin{array}{r}\text { Depth } \\
(\mathrm{km})\end{array}$} & \multicolumn{3}{|r|}{ Epicenter } & \multirow{2}{*}{$\begin{array}{c}\text { Felt } \\
\text { Report }\end{array}$} \\
\hline & & $\underline{\underline{h}}$ & $\underline{m}$ & $\underline{\mathrm{s}}$ & & & Lat. N. & Long. $\bar{W}$. & Description & \\
\hline \multirow[t]{21}{*}{$\overline{\text { July }}$} & 14 & 08 & 10 & 16.0 & 2.6 & --- & $---\cdots$ & ------ & 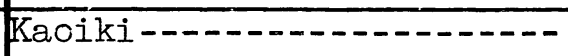 & $-1-10-1-0-0-1$ \\
\hline & 14 & 22 & 54 & 54.7 & 2.6 & -- & ------ & $---1--$ & 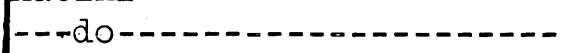 & $---1-1-1-1-1$ \\
\hline & 15 & 22 & 44 & 10.3 & 2.6 & --- & $---n--$ & ------ & Upper east rift_-...-. & $---1--1--1$ \\
\hline & 17 & 18 & 43 & 36.0 & 2.3 & --- & ------ & ------ & KM $30 \ldots$ & $------n-\cdots$ \\
\hline & 18 & 11 & 27 & 46.0 & 3.3 & 8 & $19^{\circ} 22.0^{\prime}$ & $155^{\circ} 52.3^{\prime}$ & $18 \mathrm{~km}$ SSE of Kealakekua & $\begin{array}{l}\text { Felt at } \\
\text { Honaunau. }\end{array}$ \\
\hline & 18 & 16 & 28 & 51.7 & 2.4 & --- & $----n$ & $-\cdots--n-\infty$ & KM $30-\ldots$ & $-\ldots-n-n-n$ \\
\hline & 19 & 20 & 12 & 36.5 & 2.5 & --- & ------ & 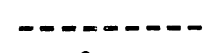 & Upper east rift-..... & 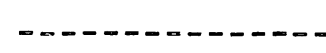 \\
\hline & 20 & ০o & 03 & 21.2 & 2.7 & 13 & $20^{\circ} 04.0^{\circ}$ & $155^{\circ} 37.2^{\prime}$ & $\begin{array}{l}10 \mathrm{~km} \text { ENE of Kamuela } \\
\text { seismograph statior. }\end{array}$ & $---1--1--1-4$ \\
\hline & 20 & 11 & 26 & 43.5 & 2.3 & -- & $-----\infty$ & -------- & Kaoiki_-_-_. & $---n-n-n-n$ \\
\hline & 20 & 12 & 31 & 31.5 & 3.2 & 13 & $19^{\circ} 10.8^{\prime}$ & $155^{\circ} 36.9^{\prime}$ & $12 \mathrm{~km}$ NNW of Naalehu-.-.-- & $-n-n-\infty-n-\infty$ \\
\hline & 21 & 17 & 24 & 02.0 & 3.2 & 13 & $19^{\circ} 59.0^{\prime}$ & $155^{\circ} 24.2^{\prime}$ & $13 \mathrm{~km} \mathrm{SE}$ of Honokaa--.--- & 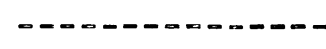 \\
\hline & 23 & 01 & 37 & OI. 0 & 2.8 & 3 & $18^{\circ} 59.0^{\prime}$ & $155^{\circ} 13.5^{\prime}$ & $32 \mathrm{~km}$ SSW of Apua Point--- & $---1-n-n-1-n$ \\
\hline & 23 & 03 & 52 & 10.8 & 3.2 & -- & $-----n$ & $--1-n-1$ & KM 30-1. & $---n---n-n$ \\
\hline & 23 & 07 & 38 & 12.6 & 2.7 & 13 & $19^{\circ} 50.9^{\prime}$ & $155^{\circ} 32.1^{\prime}$ & $13 \mathrm{~km}$ ESE of Waikii-...-- & $---1--n-n-1$ \\
\hline & 24 & 19 & 18 & 03.5 & 2.7 & 3 & $19^{\circ} 36.9^{\prime}$ & $155^{\circ} 25.8^{\prime}$ & $\begin{array}{l}14 \mathrm{~km} \text { NNW of Mauna Loa } \\
\text { seismometer. }\end{array}$ & 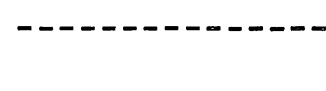 \\
\hline & 25 & 16 & 18 & 15.5 & 2.8 & 13 & $18^{\circ} 57.5^{\prime}$ & $154^{\circ} 58.2^{\prime}$ & $41 \mathrm{~km}$ SSE of Apua Point & $---n--n-n$ \\
\hline & 25 & 18 & 19 & 36.1 & 2.7 & 13 & $19^{\circ} 45.0^{\prime}$ & $155^{\circ} 58.9^{\prime}$ & $27 \mathrm{~km} \mathrm{NNW}$ of Kealakekua & $-\ldots-n-n-n$ \\
\hline & 26 & 03 & 21 & 19.7 & 2.3 & -- & ------ & $---1-n$ & 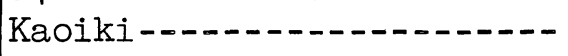 & $---1--1-n$ \\
\hline & 28 & 03 & 32 & 10.5 & 2.4 & 13 & $19^{\circ} 51.5^{\prime}$ & $155^{\circ} 38.2^{\prime}$ & $2 \mathrm{~km}$ ESE of Waikii-...... & $-1-2-1-n$ \\
\hline & 29 & 08 & 39 & 12.5 & 2.7 & -- & $----n$ & $=-------$ & Kaoikin-n-n & $---1-n-1-n$ \\
\hline & 30 & 04 & 32 & 54.0 & 2.4 & 8 & $19^{\circ} 47.5^{\prime}$ & $155^{\circ} 26.5^{\prime}$ & $\begin{array}{l}34 \mathrm{~km} \text { NNW of Mauna Loa } \\
\text { seismometer. }\end{array}$ & $-------n---$ \\
\hline \multirow[t]{4}{*}{ Aug. } & 1 & 01 & 10 & 52.4 & 2.9 & --- & --- & --- & KM $30 \ldots \ldots$ & $\begin{array}{l}\text { Felt at } \\
\text { Glenwood } \\
\text { and Volcano. }\end{array}$ \\
\hline & 2 & 10 & 06 & 29.3 & 2.1 & --- & & & KM 30- & $----1------$ \\
\hline & 2 & 14 & 53 & 03.1 & 2.8 & --- & ------- & ------- & KM 30-n- & $----1--1--1$ \\
\hline & 2 & 17 & 55 & 00.4 & 2.0 & --- & ------ & ------- & Upper east rift--n- & $---1---1--1$ \\
\hline
\end{tabular}


Table 4.--Local earthquakes recorded by seismographs of the U.S. Geological Survey, July, August, and September, 1963--Continued

\begin{tabular}{|c|c|c|c|c|c|c|c|c|c|}
\hline \multirow{2}{*}{$\begin{array}{c}\text { Date } \\
\text { (1963) }\end{array}$} & \multicolumn{3}{|c|}{ Time } & \multirow{2}{*}{$\begin{array}{c}\text { Magni- } \\
\text { tude }\end{array}$} & \multirow{2}{*}{$\begin{array}{r}\text { Depth } \\
(\mathrm{km})\end{array}$} & \multicolumn{3}{|r|}{ Epicenter } & \multirow{2}{*}{$\begin{array}{c}\text { Felt } \\
\text { Report }\end{array}$} \\
\hline & $\underline{\text { h }}$ & $\underline{m}$ & $\underline{\mathrm{s}}$ & & & Lat. N. & Long. W. & Description & \\
\hline ug. r $\begin{array}{r}3 \\
3 \\
4 \\
4 \\
4 \\
4 \\
4 \\
4 \\
4 \\
4 \\
4 \\
4 \\
5 \\
7 \\
8 \\
8 \\
8 \\
9 \\
10 \\
12 \\
14\end{array}$ & $\begin{array}{l}06 \\
23 \\
23 \\
00 \\
00 \\
01 \\
01 \\
02 \\
03 \\
07 \\
07 \\
08 \\
08 \\
07 \\
16 \\
16 \\
22 \\
18 \\
04 \\
11 \\
05\end{array}$ & $\begin{array}{l}41 \\
21 \\
47 \\
10 \\
54 \\
13 \\
39 \\
57 \\
45 \\
17 \\
54 \\
53 \\
15 \\
54 \\
13 \\
14 \\
04 \\
41 \\
10 \\
09 \\
25\end{array}$ & $\begin{array}{l}22.0 \\
02.5 \\
35.0 \\
49.5 \\
11.7 \\
48.1 \\
54.9 \\
06.5 \\
53.0 \\
40.5 \\
58.6 \\
35.3 \\
22.1 \\
23.0 \\
55.2 \\
20.0 \\
14.1 \\
43.0 \\
08.0 \\
42.6 \\
38.1 \\
38.4 \\
19.0 \\
41.3 \\
00.1 \\
20.5\end{array}$ & $\begin{array}{l}2.6 \\
2.0 \\
2.0 \\
2.7 \\
2.3 \\
2.3 \\
2.0 \\
2.0 \\
2.3 \\
2.0 \\
2.3 \\
3.4 \\
2.5 \\
2.9 \\
2.2 \\
3.3 \\
2.1 \\
2.4 \\
2.5 \\
2.2 \\
3.5 \\
3.4 \\
2.3 \\
2.2 \\
2.8 \\
2.9\end{array}$ & $\begin{array}{c}8 \\
--- \\
--- \\
--- \\
--- \\
--- \\
--- \\
--- \\
3 \\
--- \\
--- \\
13 \\
--- \\
13 \\
--- \\
--- \\
--- \\
--- \\
--- \\
--- \\
30 \\
\text { shallow } \\
--- \\
--- \\
--- \\
13 \\
13 \\
13 \\
--- \\
---\end{array}$ & 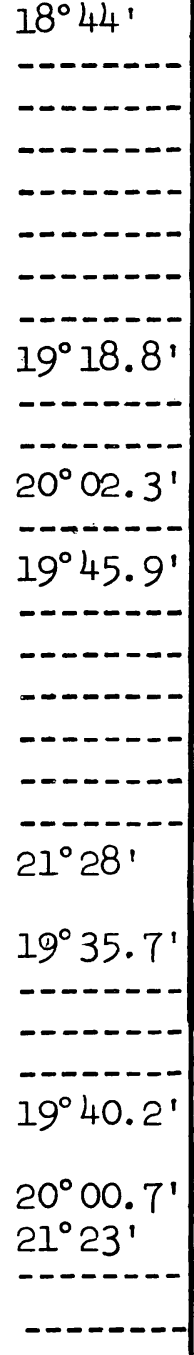 & $\begin{array}{l}154^{\circ} 57^{\prime} \\
155^{\circ} 0.5^{\prime} \\
155^{\circ} 18.8 \\
156^{\circ} 00.8 \\
158^{\circ} 06^{\prime} \\
155^{\circ} 58.8 \\
155^{\circ} 49.8 \\
156^{\circ} 29^{\prime} \\
-156^{\circ} 02.9^{\prime} \\
-2\end{array}$ & 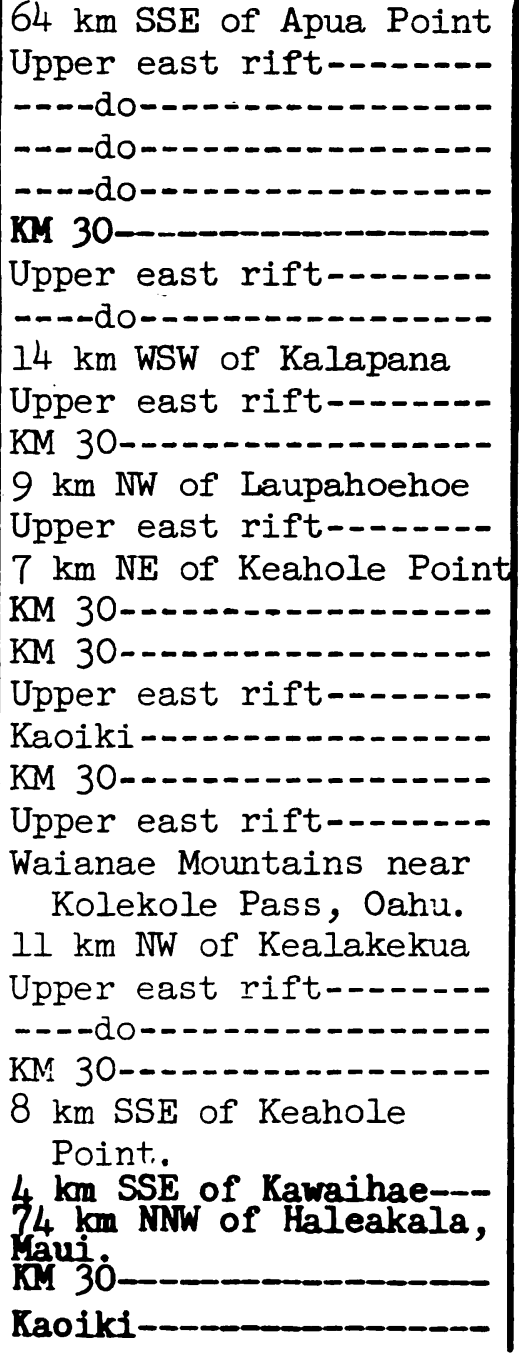 & Felt in Oahu \\
\hline
\end{tabular}


Table 4. - - Local earthquakes recorded by seismographs of the U.S. Geological Survey, July, August, and September, 1963--Continued

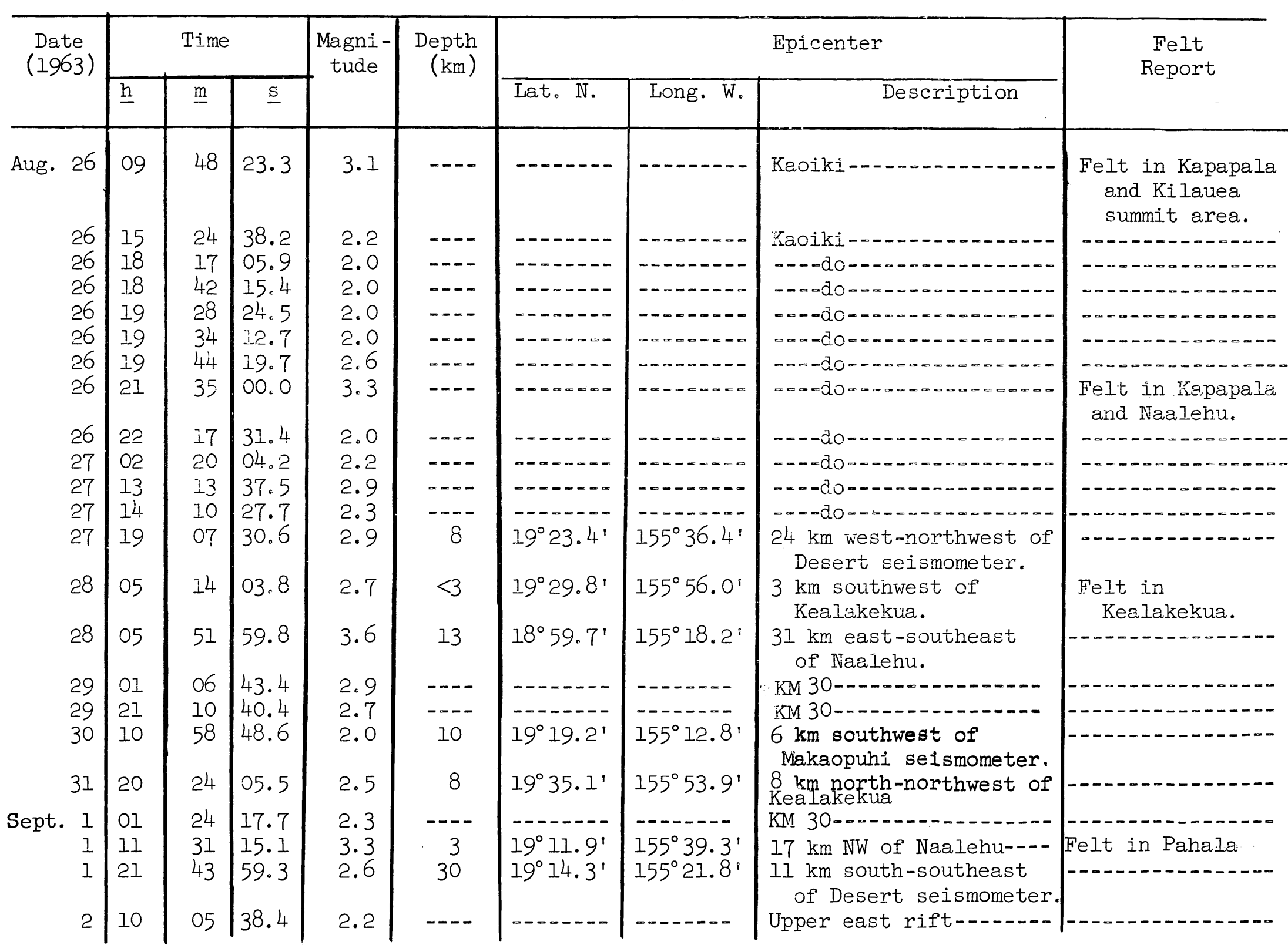


Table 4. --Local earthquakes recorded by seismographs of the U.S. Geological Survey, JuIy, August, and September, 1963--Continued

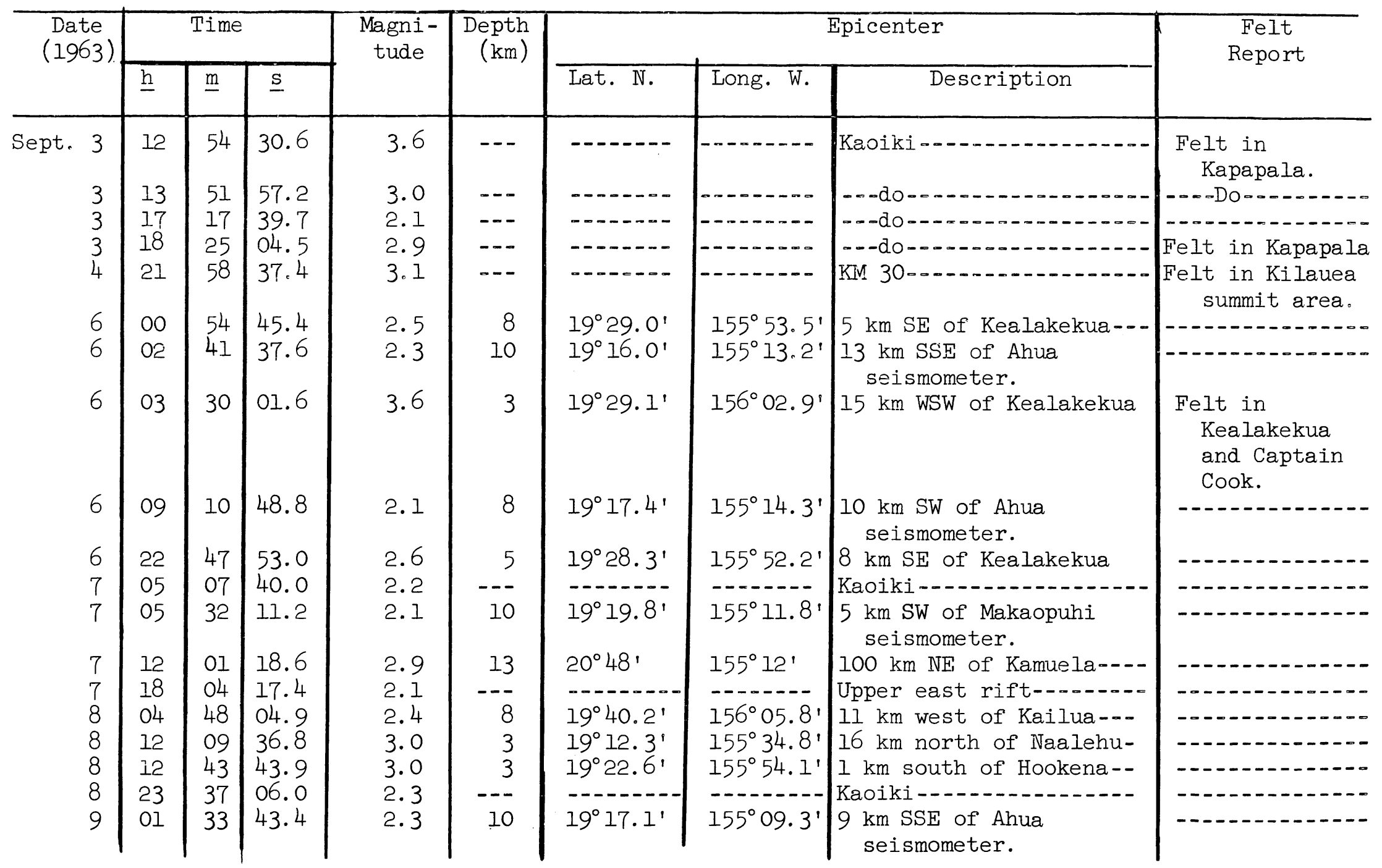


Table 4. - -Local earthquakes recorded by seismographs of the U.S. Geological Survey, July, August, and September, 1963--Continued

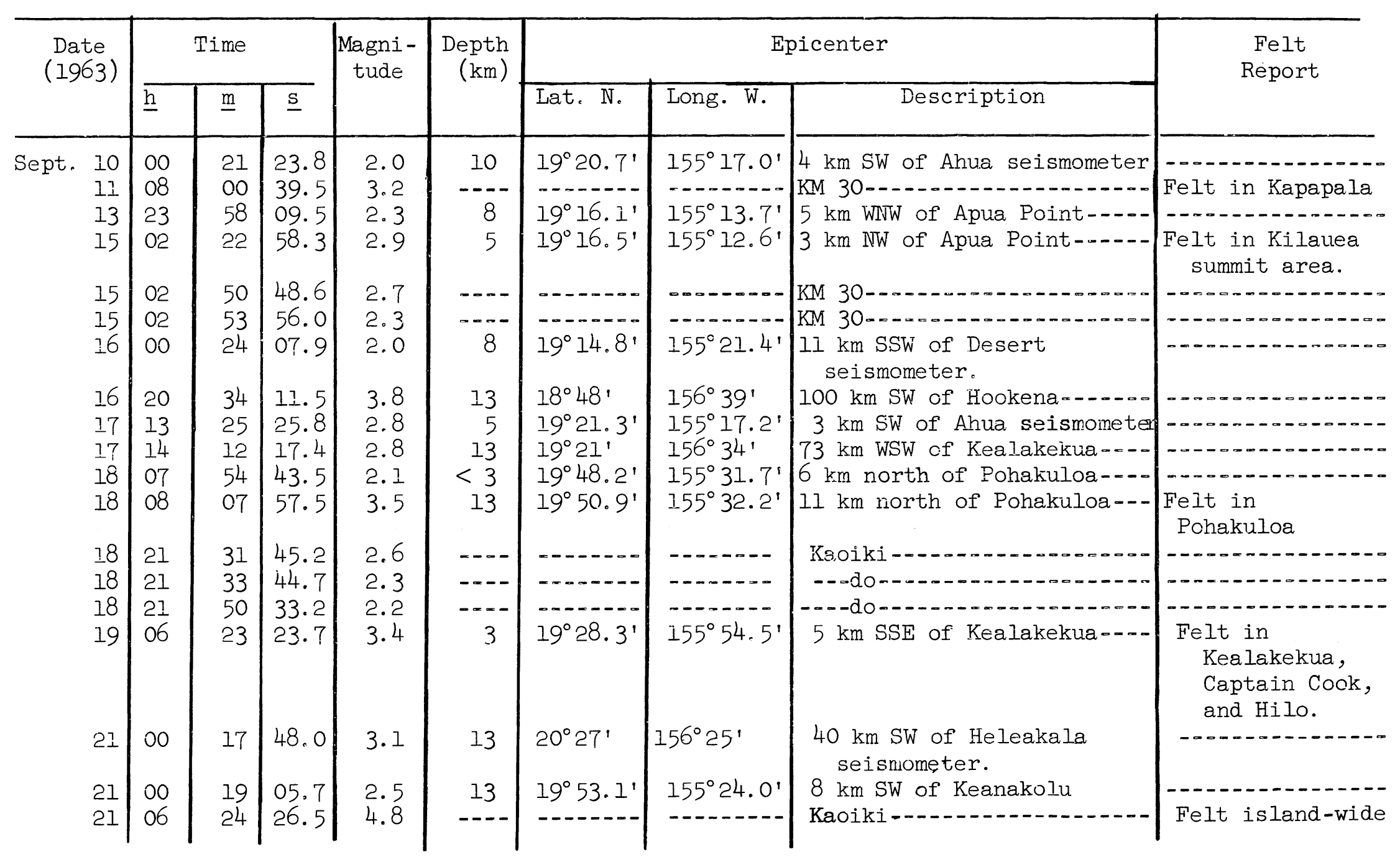


Table 4. --Local earthquakes recorded by seismographs of the U.S. Geological Survey, July, August, and September, 1963--Continued

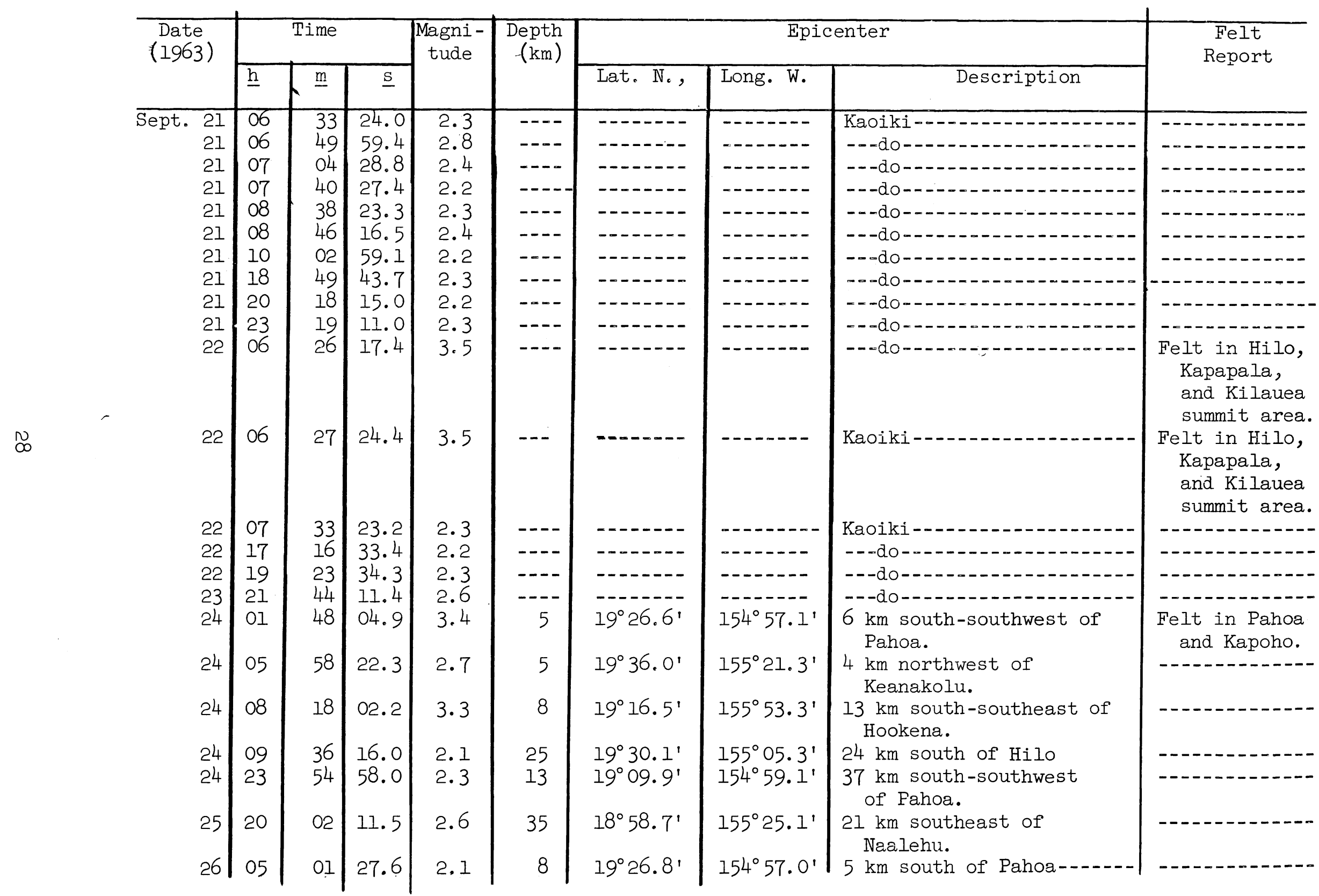


Table 4.--Iocal earthquakes recorded by seismographs of the U.S. Geological Survey, July, August, and September, 1963--Continued

\begin{tabular}{|c|c|c|c|c|c|c|c|c|c|}
\hline \multirow{2}{*}{$\begin{array}{l}\text { Date } \\
\text { (1963) }\end{array}$} & \multicolumn{3}{|c|}{ Time } & \multirow{2}{*}{$\begin{array}{l}\text { Magni- } \\
\text { tude }\end{array}$} & \multirow{2}{*}{$\begin{array}{l}\text { Depth } \\
(\mathrm{km})\end{array}$} & \multicolumn{3}{|c|}{ Epicenter } & \multirow{2}{*}{$\begin{array}{c}\text { Felt } \\
\text { Report }\end{array}$} \\
\hline & $\underline{h}$ & $\underline{m}$ & $\underline{\mathrm{s}}$ & & & Lat. N. & Long. W. & Description & \\
\hline Sept. 2 & $\begin{array}{l}17 \\
20 \\
02 \\
10\end{array}$ & $\begin{array}{l}09 \\
04 \\
45 \\
18\end{array}$ & $\begin{array}{l}24.7 \\
20.0 \\
33.9 \\
10.6\end{array}$ & $\begin{array}{l}2.9 \\
2.3 \\
3.0 \\
2.4\end{array}$ & $\begin{array}{r}3 \\
8 \\
8\end{array}$ & $\begin{array}{l}19^{\circ} 25.8^{\prime} \\
19^{\circ} 26.8^{\prime} \\
18^{\circ} 58.2^{\prime}\end{array}$ & $\begin{array}{l}154^{\circ} 57.3^{\prime} \\
154^{\circ} 56.1^{\prime} \\
155^{\circ} 18.0^{\prime}\end{array}$ & $\begin{array}{l}7 \mathrm{~km} \text { south-southwest of } \\
\text { Pahoa. } \\
\text { Kaoiki-a } \\
6 \mathrm{~km} \text { south of Pahoa-. } \\
5 \mathrm{~km} \text { south of } \\
\text { Uwekahuna } \\
\text { seismometer. } \\
7 \mathrm{~km} \text { southeast of Pahoa. }\end{array}$ & $\begin{array}{l}\text { Felt in Pahoa } \\
\text { Felt in Kapoho } \\
-\infty\end{array}$ \\
\hline
\end{tabular}




\section{Table 5.--Distant earthguakes}

[Times are reported in Greenwich Civil Time which is 10 hours faster than Hawaiian Standard Time. A " $c$ " following the time of $P$ indicates compressional first motion; a "d" indicates àilatational first motion. Station symbols, locations, and instrumentation are presented in Summary 28. Magnitudes calculated from the Hawaii seismograms are followed by (HVO). Location of epicenter, origin times, and focal depths, and magnitudes reported by other institutions are taken from "Preliminary Determination of Epicenters" published by the U.S. Coast and Geodetic Survey ]

\begin{tabular}{|c|c|c|c|}
\hline \multicolumn{4}{|c|}{ July 4, 1963} \\
\hline M & Z & $\mathrm{eP}$ & $11: 06: 56.7 \mathrm{~d}$ \\
\hline A & Z & $\mathrm{eP}$ & $56.4 \mathrm{~d}$ \\
\hline $\mathrm{D}$ & Z & $\mathrm{eP}$ & $55.4 \mathrm{~d}$ \\
\hline$M P$ & Z & eP & $56.4 \mathrm{~d}$ \\
\hline $\mathrm{U}$ & Z & iP & $56.6 \mathrm{~d}$ \\
\hline $\mathrm{Pa}$ & Z & iP & 58.1 a \\
\hline $\mathrm{Na}$ & Z & iP & $53.3 \mathrm{~d}$ \\
\hline $\mathrm{Ka}$ & Z & iP & $59.1 \mathrm{~d}$ \\
\hline $\mathrm{Hi}$ & $\mathrm{Z}$ & iP & $59.1 \mathrm{~d}$ \\
\hline $\mathrm{Ha}$ & Z & iP & $11: 07: 03.1 \mathrm{~d}$ \\
\hline $\mathrm{U}$ & PEZ & iP & $11: 06: 56 \quad \mathrm{~d}$ \\
\hline $\mathrm{U}$ & PENT & iS & $11: 13: 59$ \\
\hline
\end{tabular}

C\&GS card 54-63:

10:58:13.2

$26.3^{\circ} \mathrm{S} ., 177.7^{\circ} \mathrm{W}$.

Tonga Is lands region

$\mathrm{h}$ about $158 \mathrm{~km}$

Magnitude 6.75 (Pas)

6.75 to 7 (Brk)

6.5 (CGS).

\section{July 9}

U PEZ eR 09:57:02

C\&GS card 55-63:

$09: 24: 33.3$

$8.5^{\circ}$ N., $83.0^{\circ}$ W.

Costa Rica

Panama Border

$\mathrm{h}$ about $31 \mathrm{~km}$

Magnitude 5.1 (CGS).

\section{July 10}

$\begin{array}{lllr}M & Z & \text { iP } & 05: 31: 49.4 \mathrm{~d} \\ \mathrm{~N} & \mathrm{Z} & \mathrm{eP} & 50.2 \mathrm{~d} \\ \mathrm{~Pa} & \mathrm{Z} & \mathrm{eP} & 49.7 \mathrm{~d}\end{array}$

July 10---Continued

\begin{tabular}{|c|c|c|c|}
\hline $\mathrm{U}$ & PEZ & iP & 49 \\
\hline $\mathrm{U}$ & PEZ & iR & $05: 45: 31$ \\
\hline U & PEE & is & $05: 39: 02$ \\
\hline U & PEN & iL & $05: 43: 26$ \\
\hline
\end{tabular}

C\&GS cara 55-63:

$05: 22: 57.1$

$46.3^{\circ}$ N., $152.9^{\circ} \mathrm{E}$.

Kurile Islands region

hl about $33 \mathrm{~km}$

Magnitude 5.6 (CGS)

6.1 (HVO).

July 14

$\begin{array}{lllr}M & Z & \text { iP } & 17: 16: 58.6 \mathrm{~d} \\ \text { A } & Z & \text { iP } & 58.1 \mathrm{~d} \\ \text { D } & Z & \text { iP } & 57.3 \mathrm{~d} \\ \text { MP } & Z & \text { iP } & 58.1 \mathrm{~d} \\ \text { U } & \text { Z } & \text { iP } & 58.3 \mathrm{~d}\end{array}$

C\&GS card 57-63:

$17: 06: 38.6$

$39.4^{\circ} \mathrm{S} ., 174.9^{\circ} \mathrm{E}$.

North Island, New Zealand

$\mathrm{h}$ about $189 \mathrm{~km}$

Magnitude 6.0 (CGS).

July 15

Ha $\mathrm{Z}$ Tmax 07:11:51

C\&CGS card 57-63:

$06: 28: 21.7$

$51.8^{\circ} \mathrm{N} ., 176.8^{\circ} \mathrm{W}$.

Andreanof Islands, Aleutian Islands $\mathrm{h}$ about $33 \mathrm{~km}$

Magnitude 4.6 (CGS). 
Table 5.--Distant earthquakes---Continued

\begin{tabular}{|c|c|c|c|}
\hline \multicolumn{4}{|c|}{ July 15} \\
\hline M & Z & iP & $08: 49: 43.3 \mathrm{~d}$ \\
\hline A & Z & eP & $45.0 \mathrm{~d}$ \\
\hline D & Z & iP & $44.3 \mathrm{~d}$ \\
\hline $\mathrm{U}$ & Z & eP & $44.1 \mathrm{~d}$ \\
\hline $\mathrm{Pa}$ & Z & iP & $44.3 d$ \\
\hline $\mathrm{Ha}$ & Z & Tme & $09: 39: 39$ \\
\hline
\end{tabular}

C\&GS card 56-63:

$08: 41: 07.5$

$55.6^{\circ}$ N., $162.0^{\circ} \mathrm{E}$.

Kamchatka

$h$ about $60 \mathrm{~km}$

Magnitude 5.2 (CGS).

July 16

$\begin{array}{llll}\text { U } & \text { PEN } & \text { ES } & 18: 56: 45 \\ \text { U } & \text { PEN } & \text { eI } & 19: 16: 33 \\ \text { U } & \text { PEZ } & \text { eR } & 19: 22: 11\end{array}$

C\&GS card 57-63:

$18: 27: 18.4$

43. $1^{\circ} \mathrm{N} ., 41.5^{\circ} \mathrm{E}$.

Georgia S.S.R.

$\mathrm{h}$ about $33 \mathrm{~km}$

Magnitude 5.8 (CGS).

July 18

$\mathrm{Ha}$ Z Tmax $00: 48: 23$

$\mathrm{Pa} \quad \mathrm{T}$ max 00:49:02

C\&GS card 57-63:

$00: 04: 05.3$

49. $1^{\circ}$ N., $128.9^{\circ} \mathrm{W}$.

Vancouver Is lands region $\mathrm{h}$ about $33 \mathrm{~km}$

Magnitude 4.8 (CGS).

\section{July 19}

$\begin{array}{llll}\text { M } & \text { Z } & T \max & 13: 28: 16 \\ \text { A } & \text { Z } & T \max & 13: 28: 00 \\ \text { D } & \text { Z } & T \max & 13: 28: 18 \\ \text { MP } & \text { Z } & T \max & 13: 27: 59 \\ \text { Ha } & \text { Z } & T \max & 13: 28: 05\end{array}$

No coast and Geodetic Survey preliminary listing.

July 24

U PEZ eR 12:07:51

C\&GS card 59-63:

$11: 32: 17.7$

$24.6^{\circ} \mathrm{IN}_{0}, 122.0^{\circ} \mathrm{E}$.

Near east coast of Formosa
July 24--Continued

h about $33 \mathrm{~km}$

Magnitude 5.3 (CGS).

July 29

U PEZ IP 20:23:30 d

U PEZ IR 20:38:20

U PEE IS 20:31:00

U PEN IG 20:35:56

C\&GS card 61-63:

$20: 14: 07.3$

$30.2^{\circ} \mathrm{S} ., 177.3^{\circ} \mathrm{W}$.

Kermadec Islands

$\mathrm{h}$ about $39 \mathrm{~km}$

Magnitude $6.5-6.75$ (Bks)

5.7 (CGS)

6.5 (HVO).

July 30

\begin{tabular}{|c|c|c|c|}
\hline $\mathrm{M}_{\mathrm{I}}$ & Z & iP & $05: 55: 16.0 \mathrm{~d}$ \\
\hline A & $\mathrm{Z}$ & iP & 15.5 \\
\hline D & Z & $e P$ & 14.9 \\
\hline $\mathrm{U}$ & PEZ & iP & 16 \\
\hline
\end{tabular}

U PEZ IR 06:10:28

U PEN IS 06:02:52

U PEN EG 06:07:40

C\&GS card 59-63:

$05: 45: 53.3$

$29.6^{\circ} \mathrm{S} ., 177.3^{\circ} \mathrm{W}$.

Kermadec Islands region

$\mathrm{h}$ about $33 \mathrm{~km}$

Magnitude 5.3 (CGS)

6. 1 (HVO).

Aug. 2

M Z iP 19:38:19.5 d

C\&GS card 62-63:

$19: 26: 26.0$

$6.0^{\circ}$ N., $125.1^{\circ} \mathrm{E}$.

Mindarao, Philippine Is lands

$\mathrm{h}$ about $118 \mathrm{~km}$

Magnitude 5.0 (CGS). 
Table 5.--Distant earthquakes--Continued

$\frac{\text { August } 3}{\mathrm{~Pa} \quad \mathrm{Z} \quad \mathrm{Tmax} \text { 02:17:26 }}$
C\&GS card 67-63:
01:09:56
8.8 $\mathrm{S}$., 108. $3^{\circ} \mathrm{W}$.
Southwest of Galapagos Islands
h about $33 \mathrm{~km}$
Magnitude 4.4 (CGS).

August 3

$\begin{array}{llll}U & \text { PEZ } & \text { ePP } & 10: 41: 26 \\ U & \text { PEZ } & \text { ISS } & 10: 56: 45 \\ U & \text { PEE } & \text { IPS } & 10: 50: 53 \\ U & \text { PEE } & \text { IL } & 11: 07: 53\end{array}$

C\&GS card 61-63:

$10: 21: 36.6$

$7.7^{\circ}$ N., $35.8^{\circ} \mathrm{W}$.

Mid-Atlantic Ocean

$\mathrm{h}$ about $33 \mathrm{~km}$

Magnitude 6.1 (CGS)

6.3 (HVO).

\section{August 3}

$\begin{array}{lllr}\text { M } & \text { Z } & \text { iP } & 20: 35: 30.5 \mathrm{~d} \\ \mathrm{~A} & \mathrm{Z} & \mathrm{eP} & 30.3 \mathrm{~d} \\ \mathrm{D} & \mathrm{Z} & \mathrm{eP} & 29.0 \mathrm{~d} \\ \mathrm{Hi} & \mathrm{Z} & \mathrm{eP} & 33.1 \mathrm{~d}\end{array}$

C\&GS card 62-63:

$20: 26: 04.1$

$30.7^{\circ} \mathrm{S} ., 178.3^{\circ} \mathrm{W}$.

Kermadec Islands

$\mathrm{h}$ about $37 \mathrm{~km}$

Magnitude 5.2 (CGS).

August $4-5$

$\begin{array}{lllr}\text { M } & Z & \text { iP } & 00: 01: 33.9 \mathrm{c} \\ \text { A } & \text { Z } & \text { iP } & 33.8 \mathrm{c} \\ \text { D } & Z & \text { eP } & 33.6 \mathrm{c} \\ \text { MP } & \text { Z } & \text { iP } & 33.8 \mathrm{c}\end{array}$

August 4-5--Continued

$\begin{array}{llll}U & Z & \text { iP } & 33.7 \mathrm{c} \\ \mathrm{Pa} & \mathrm{Z} & \text { eP } & 34.8 \mathrm{~d} \\ \mathrm{Na} & \mathrm{Z} & \text { iP } & 30.5 \mathrm{c} \\ \mathrm{Ka} & \mathrm{Z} & \text { iP } & 36.0 \mathrm{c} \\ \mathrm{Hi} & \mathrm{Z} & \text { iP } & 35.4 \mathrm{~d} \\ \mathrm{Ha} & \text { Z } & \text { iP } & 38.5 \mathrm{~d}\end{array}$

CSOGS card 62-63:

$23: 54: 14.0$

$17.5^{\circ} \mathrm{S} ., 179.1^{\circ} \mathrm{W}$.

Fiji Islands region

h about $515 \mathrm{~km}$

Magnitude 5.2 (CGS).

August 8

$\begin{array}{lllr}M & Z & \text { eP } & 02: 23: 04.6 \mathrm{c} \\ \mathrm{D} & \mathrm{Z} & \text { iP } & 05.2 \mathrm{c} \\ \mathrm{U} & \mathrm{Z} & \text { iP } & 05.0 \mathrm{c} \\ \mathrm{Pa} & \mathrm{Z} & \text { iP } & 06.0 \mathrm{c} \\ \mathrm{Hi} & \mathrm{Z} & \mathrm{eP} & 03.7 \mathrm{c} \\ \mathrm{U} & \mathrm{PETN} & \mathrm{eS} & 02: 29: 40 \\ \mathrm{U} & \mathrm{PEE} & \mathrm{eL} & 02: 33: 04 \\ \mathrm{U} & \mathrm{PEZ} & \text { iR } & 02: 35: 11\end{array}$

C\&ISS card 63-63:

$02: 14: 54.4$

$54.2^{\circ}$ N., $168.1^{\circ} \mathrm{E}$.

Fox Islands, Aleutian Islands

$\mathrm{h}$ about $33 \mathrm{~km}$

Magnitude 5.5 (CGS)

5.8 (HVO).

August 8

M Z eP 11:26:04.9 d

A Z eP $\quad 05.0 \mathrm{~d}$

D Z iP $\quad 04.4 \mathrm{~d}$

$\begin{array}{llll}U & \mathrm{Z} & \mathrm{e} & 05.0 \mathrm{c}\end{array}$

INa Z eP O2.I c

$\mathrm{Hi} Z$ iP $07.0 \mathrm{c}$

$\mathrm{Ha} \quad \mathrm{Z}$ iP $08.1 \mathrm{~d}$

$\mathrm{U}$ PEZ eP O5 c

U P I IR 11:43:04

$\mathrm{U}$ PEE ES 11:34:09

$\mathrm{U}$ PEIS $\mathrm{EL}$ 11:40:04 
Table 5.--Distant earthquakes--Continued

August 8, 1963-..Continued

C\&GS carả 65-53:

$11: 16: 1.1 .2$

$5.8^{\circ}$ S., 151. $0^{\circ}$.

New Britain

$\mathrm{h}$ about $48 \mathrm{~km}$

Felt: Palmalmal, Pomio

Magnitude 5 (Bks)

$$
\text { 5.6 (CGS). }
$$

Aug. 9

$\begin{array}{llll}\text { U } & \text { PEZ } & \text { eP } & 14: 44: 14 c \\ U & \text { PEZ } & \text { IS } & 14: 50: 34 \\ U & \text { PEZ } & \text { IR } & 14: 54: 39 \\ U & \text { PEE } & \text { IL } & 14: 52: 56\end{array}$

C\&GS card 64.63:

$14: 36: 45.9$

$15.3^{\circ}$ S., $175.7^{\circ} \mathrm{W}$.

Fiji Islands region

$\mathrm{h}$ about $33 \mathrm{~km}$

Magnitude 5.5 (CGS )

5.7 (HVO).

Aug. 14

$\begin{array}{llrr}M & Z & \text { iP } & 18: 55: 18.8 \mathrm{~d} \\ \mathrm{~A} & \mathrm{Z} & \text { iP } & 18.0 \mathrm{~d} \\ \mathrm{Hi} & \mathrm{Z} & \mathrm{eP} & 19.6 \mathrm{c}\end{array}$

C\&GS card 64.53 :

$18: 43: 55.5$

$3.4^{\circ} \mathrm{S} ., 135.4^{\circ} \mathrm{E}$.

West Iran

h about $33 \mathrm{~km}$

Magnitude 6.4 (CGS)。

Aug. 15

\begin{tabular}{|c|c|c|c|}
\hline M & $\mathrm{Z}$ & $e P$ & $06: 21: 17.2 \mathrm{c}$ \\
\hline A & Z & eP & $20.0 d$ \\
\hline D & Z & $e P$ & 17.5 \\
\hline $\mathrm{Pa}$ & Z & $\mathrm{eP}$ & 19.3 \\
\hline $\mathrm{Hi}$ & Z & iP & 18.3 \\
\hline $\mathrm{Ha}$ & Z & $\mathrm{eP}$ & 15.00 \\
\hline $\mathrm{U}$ & PEZ & iP & 18 \\
\hline & PEZ & $i R$ & $06: 37: 54$ \\
\hline & PEE & iS & $06: 29: 15$ \\
\hline
\end{tabular}

August 15--Continued

C\&GS caro. 64-63:

$06: 11: 34.6$

$37.9^{\circ} \mathrm{N} ., 141.6^{\circ} \mathrm{E}$.

Near east coast of Honshu, Japan

h about $59 \mathrm{~km}$

Ma.gnitude 6.6 (HVO).

Aulg. 15

A Z iP $17: 37: 14.5 \mathrm{~d}$

$\begin{array}{llll}\mathrm{A} & \mathrm{Z} & \mathrm{P} & 14.9 \mathrm{~d}\end{array}$

$N \quad$ Z iP $\quad 15.0 \mathrm{~d}$

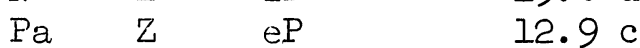

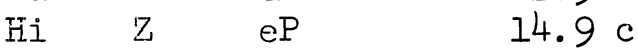

$\mathrm{U} \quad \mathrm{PF}$ eP 15 d

U PEZ ipP 17:39:21 d

U PEZ IPF 17:41:12

$U$ PEZ ipPP 17:43:00

U PEZ IPPP 17:43:27

U PEZ ISKS 17:47:04

$U$ PEZ iS 17:47:44

U PEZ iSP 17:48:38

U PEZ i 17:52:37

U PEZ ISS 17:54:04

U PEZ ISSS 17:57:02

U P.EZ i 18:08:26

U PEN isP $17: 39: 53$

U PEN ESS 17:50:59

U PEN e 18:00:27

U PEN IG 18:02:57

$U$ PEN $i \quad 18: 14: 28$

C\&GS cara 65-63:

$17: 25: 05.9$

$13.8^{\circ} \mathrm{S} ., 69.3^{\circ} \mathrm{W}$.

Peru-Bolivia border

$h$ about $543 \mathrm{~km}$

Magnitude 7.75 (Pas)

Aug. 17

M Z eP 11:23:20.9 d

$\begin{array}{llll}A & Z & \mathrm{eP} & 21.8 \mathrm{c}\end{array}$

D $Z$ eP $\quad 21.5 \mathrm{c}$

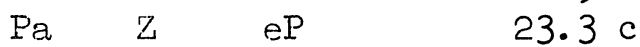

$\mathrm{Hi} \quad \mathrm{Z} \quad \mathrm{eP} \quad 21.0 \mathrm{~d}$

U PE⿱ $\quad$ iP $2 l$ d

U PEZ IR 11:42:33 
August 17, 1963--Continued

$\begin{array}{llll}\text { U } & \text { PEE } & \text { iS } & 11: 32: 17 \\ \text { U } & \text { PEN } & \text { IG } & 11: 39: 49\end{array}$

C\&GS card 66-63:

$11: 12: 41.2$

$30.6^{\circ} \mathrm{N} ., 130.9^{\circ} \mathrm{E}$.

Ryukyu Islands region

$\mathrm{h}$ about $33 \mathrm{~km}$

Magnitude 5.6 (CGS)

6.5 (HVO).

Aug. 17

$\begin{array}{lllr}M & Z & \text { iP } & 11: 43: 48.3 \mathrm{c} \\ \mathrm{A} & \mathrm{Z} & \text { iP } & 47.4 \mathrm{c} \\ \mathrm{D} & \mathrm{Z} & \text { iP } & 48.3 \mathrm{c} \\ \mathrm{U} & \mathrm{Z} & \text { iP } & 47.8 \mathrm{c} \\ \mathrm{Pa} & \mathrm{Z} & \text { iP } & 44.8 \mathrm{c}\end{array}$

C\&GS card 67-63:

$11: 34: 23.4$

$17.7^{\circ}$ N., $94.3^{\circ} \mathrm{W}$.

Veracruz, Mexico

$\mathrm{h}$ about $163 \mathrm{~km}$

Magnitude 4.9 (CGS).

Aug. 18

$\begin{array}{lllr}\text { M } & Z & \mathrm{eP} & 18: 50: 10.2 \mathrm{c} \\ \mathrm{A} & \mathrm{Z} & \mathrm{iP} & 12.2 \mathrm{c} \\ \mathrm{U} & \mathrm{Z} & \mathrm{eP} & 11.3 \mathrm{c} \\ \mathrm{Hi} & \mathrm{Z} & \mathrm{iP} & 10.4 \mathrm{c} \\ \mathrm{U} & \mathrm{PEE} & \mathrm{eL} & 18: 58: 00 \\ \mathrm{U} & \mathrm{PEZ} & \mathrm{eR} & 18: 59: 33\end{array}$

C\&GS card 65-63:

$18: 43: 16.1$

$50.3^{\circ}$ N., $176.9^{\circ} \mathrm{W}$.

Andreanof Islands, Aleutian Islands

$\mathrm{h}$ about $33 \mathrm{~km}$

Magnitude 5.5 (CGS).

Aug. 20

U PEZ eR 16:13:59
Aug. 20--Continued

C\&GS card 65-63:

$15: 48: 12.2$

$41.2^{\circ}$ N., $142.7^{\circ} \mathrm{E}$.

Off east coast of Honshu, Japan

$h$ about $50 \mathrm{~km}$

Magnitude 4.5 (CGS).

Aug. 22

Ha Z Tmax 10:09:25

C\&GS card 68-63:

$09: 27: 09.3$

42. $0^{\circ}$ N., $126.2^{\circ} \mathrm{W}$.

Off coast of Oregon

$\mathrm{h}$ about $33 \mathrm{~km}$

Magnitude 5.6 (CGS).

Aug. 22

\begin{tabular}{|c|c|c|c|}
\hline M & Z & iP & $20: 01: 47.4 \mathrm{c}$ \\
\hline $\mathrm{D}$ & Z & iP & $46.9 c$ \\
\hline $\mathrm{U}$ & $\mathrm{Z}$ & $e P$ & 47.2 \\
\hline $\mathrm{Pa}$ & $\mathrm{Z}$ & $\mathrm{eP}$ & 50.2 \\
\hline $\mathrm{Na}$ & 乙 & eP & 45.1 \\
\hline $\mathrm{Hi}$ & $\mathrm{Z}$ & iP & 50.5 \\
\hline $\mathrm{Ha}$ & $\mathrm{Z}$ & $e P$ & 51.1 \\
\hline U & PEZ & eP & 50 \\
\hline U & PEZ & $i R$ & $20: 16: 46$ \\
\hline U & $\mathrm{PEE}$ & is & $20: 09: 26$ \\
\hline
\end{tabular}

C\&GS card 69-63:

$19: 52: 25.0$

$9.4^{\circ} \mathrm{S} ., 158.0^{\circ} \mathrm{E}$.

Solomon Islands

Felt

h about $33 \mathrm{~km}$

Magnitude 6.75-7 (Pas)

6.1 (CGS)

6.6 (HVO) 
Table 5. --Distant earthquakes--Continued

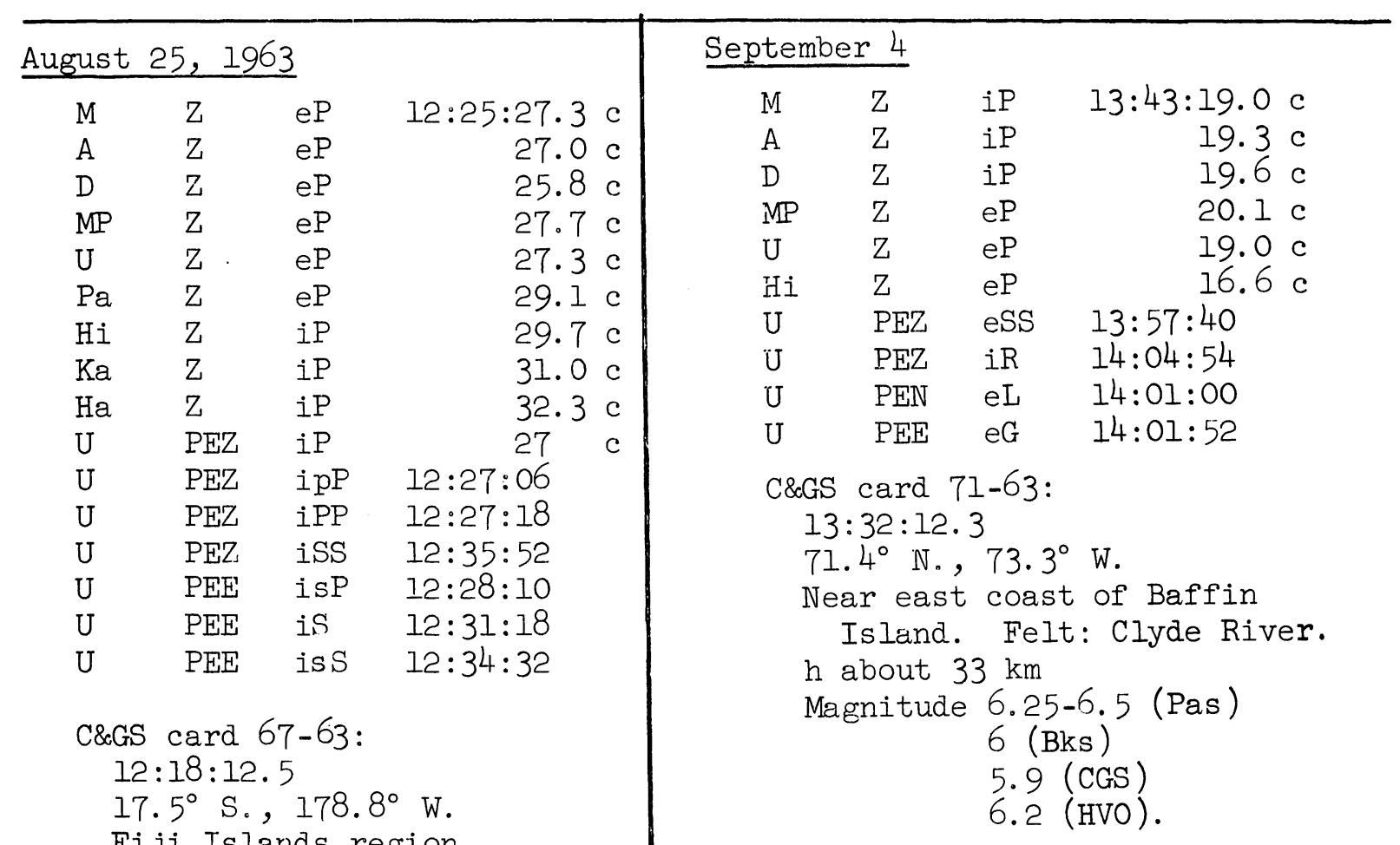

Sept. 6

$\mathrm{h}$ about $565 \mathrm{~km}$

Magnitude 6.5 (Pas) 6-6.25 (Brk)

6.1 (CGS)

6.5 (HVO).

Aug. 29

\begin{tabular}{|c|c|c|c|}
\hline M & Z & iP & $15: 42: 27.30$ \\
\hline A & Z & eP & 26.7 \\
\hline D & Z & $\mathrm{eP}$ & 26.70 \\
\hline $\mathrm{U}$ & Z & iP & $26.8 \mathrm{c}$ \\
\hline $\mathrm{Pa}$ & Z & iP & 25.10 \\
\hline $\mathrm{Na}$ & Z & iP & 23.8 \\
\hline $\mathrm{Hi}$ & Z & eP & 27.7 \\
\hline $\mathrm{Ha}$ & Z & $e P$ & 33.5 \\
\hline $\mathrm{U}$ & PEZ & iP & 27 \\
\hline U & PEZ & iS & $15: 52: 58$ \\
\hline U & PEZ & iSSS & $16: 02: 50$ \\
\hline U & PEZ & $i G$ & 16:06:02 \\
\hline
\end{tabular}

C\&GS card 68-63:

$15: 30: 31.4$

$7.1^{\circ}$ S., $81.6^{\circ} \mathrm{W}$.

Off coast of Peru

$\mathrm{h}$ about $23 \mathrm{~km}$

Magnitude 6.5 (Pas)

6.1 (CGS)

6.8 (HVO).

$\begin{array}{lllc}M & Z & \text { eP } & 21: 03: 51.9 \mathrm{~d} \\ M & Z & T m a x & 21: 41: 02 \\ M P & Z & T \max & 14 \\ U & Z & T m a x & 12 \\ \mathrm{~Pa} & \mathrm{Z} & T \max & 21: 41: 15 \\ \mathrm{Hi} & \mathrm{Z} & T \max & 21: 40: 53 \\ \mathrm{Ha} & \mathrm{Z} & T \max & 21: 39: 27\end{array}$

C\&GS card 72-63:

$20: 56: 59.9$

$53.9^{\circ}$ N., $165.6^{\circ} \mathrm{W}$.

Fox Islands, Aleutian Islands $\mathrm{h}$ about $33 \mathrm{~km}$

Magnitude 5.0 (CGS).

Sept. 8

$\begin{array}{lllr}M & Z & \text { eP } & 19: 58: 28.5 \mathrm{c} \\ \mathrm{A} & \mathrm{Z} & \mathrm{eP} & 28.2 \mathrm{c} \\ \mathrm{MP} & \mathrm{Z} & \mathrm{eP} & 28.3 \mathrm{c} \\ \mathrm{U} & \mathrm{Z} & \mathrm{iP} & 28.3 \mathrm{c} \\ \mathrm{Pa} & \mathrm{Z} & \mathrm{iP} & 29.9 \mathrm{c} \\ \mathrm{Ka} & \mathrm{Z} & \text { iP } & 31.9 \mathrm{c} \\ \mathrm{Hi} & \mathrm{Z} & \text { iP } & 31.1 \mathrm{c} \\ \mathrm{Ha} & \mathrm{Z} & \text { iP } & 33.9 \mathrm{c}\end{array}$




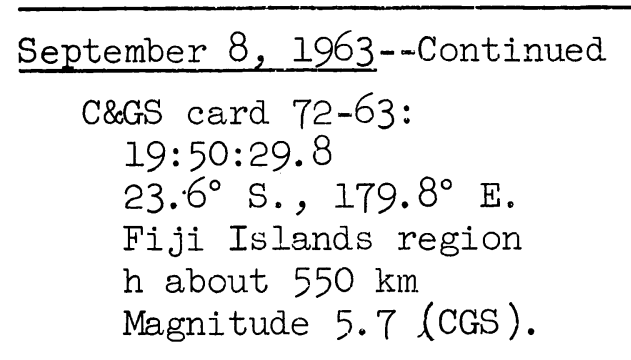

\section{$\frac{\text { Sept. } 9}{M}$}

$\mathrm{Hi}, \mathrm{Z} \quad \mathrm{eP}$

$\mathrm{Ha} \quad \mathrm{Z} \quad \mathrm{EP}$

$U$ PEZ IP

U PEZ eG

U PEZ eR

U PEE eS

M Z Tmax

A Z T $\quad$ max

D Z T $\quad$ max

U Z Tmax

Ka Z Tmax

$\mathrm{Ha} \quad \mathrm{T}$ Tmax

$02: 55: 29.1 \mathrm{c}$

$29.3 \mathrm{c}$

$31.2 \mathrm{c}$

$03: 03: 34$

$03: 11: 52$

$03: 03: 17$

$03: 55: 51$

38

48

48

$03: 56: 02$

$03: 56: 27$

C\&GS card 72-63:

$02: 45: 45.5$

$4.4^{\circ} \mathrm{S}, 152.7^{\circ} \mathrm{E}$.

New Britain;

felt, Rabaul

$h$ about $3 \mathrm{~km}$

Magnitude 5.6 (CGS)

$$
6.3 \text { (HVO). }
$$

Sept. 11

$$
\text { M Z eP 09:11:23.4 c }
$$

C\&GS card 75-63:

$08: 59: 37.6$

$3.5^{\circ} \mathrm{S} ., 131.2^{\circ} \mathrm{E}$.

Ceram

$\mathrm{h}$ about $33 \mathrm{~km}$

Magnitude 5.7 (CGS).

Sept. 13

$\begin{array}{lllr}M & Z & \mathrm{eP} & 17: 07: 25.4 \mathrm{c} \\ \mathrm{A} & \mathrm{Z} & \mathrm{eP} & 24.8 \mathrm{c} \\ \mathrm{D} & \mathrm{Z} & \mathrm{eP} & 26.3 \mathrm{c}\end{array}$

Sept. 13--Continued

$\begin{array}{llll}\mathrm{MP} & \mathrm{Z} & \mathrm{eP} & 24.3 \mathrm{c} \\ \mathrm{U} & \mathrm{Z} & \mathrm{eP} & 25.1 \mathrm{c} \\ \mathrm{Pa} & \mathrm{Z} & \mathrm{eP} & 22.4 \mathrm{c} \\ \mathrm{Na} & \mathrm{Z}_{1} & \mathrm{eP} & 29.6 \mathrm{~d} \\ \mathrm{Hi} & \mathrm{Z} & \mathrm{eP} & 21.9 \mathrm{c} \\ \mathrm{Ha} & \mathrm{Z} & \mathrm{eP} & 24.2 \mathrm{~d}\end{array}$

No C\&GS preliminary listing.

Sept. 14

$\begin{array}{lllr}M & Z & \text { iP } & 04: 01: 53.8 \mathrm{~d} \\ \mathrm{~A} & \mathrm{Z} & \mathrm{eP} & 53.3 \mathrm{~d} \\ \mathrm{MP} & \mathrm{Z} & \mathrm{eP} & 52.9 \mathrm{~d}\end{array}$

$U$ PEZ IR 04:17:16

C\&GS card 77-63:

$03: 52: 16.9$

$31.4^{\circ} \mathrm{S}, 179.0^{\circ} \mathrm{W}$.

Kermadec Islands

h about $33 \mathrm{~km}$

Magriitude 4.9 (CGS)

5.9 (HVO).

Sept。 15

$M \quad Z$ IP $00: 55: 35.5 \mathrm{~d}$

A $Z$ iP

D Z iP

U $\quad$ Z iP

$\mathrm{Pa} Z$ iP

$\mathrm{Na} \quad \mathrm{Z}$ iP

$\mathrm{Ka} \quad \mathrm{Z} \quad \mathrm{eP}$

$\mathrm{Hi} \quad \mathrm{Z} \quad \mathrm{iP}$

$\mathrm{Ha} Z$ IP

U PEZ IP

$U$ PEZ iS

U PEZ IR

U PEN $\quad I G$

A Z T Tmax 19

D Z Tmax 22

IJ Z Tmax 21

$\mathrm{Ha} \quad \mathrm{Z} \quad \mathrm{Tmax}$ 01:47:51

C\&acs card 75-63:

$00: 46: 54.1$

$10.3^{\circ} \mathrm{S} ., 165.6^{\circ} \mathrm{E}$.

Santa Cruz Islands. Felt, Vanikoro.

$\mathrm{h}$ about $33 \mathrm{~km}$

Magnitude $7.25-7.5$ (Pas)

6.3 (CGS)
$6.75-7$ (Pal)
7.5 (HVO).
$35.4 \mathrm{~d}$

$34.4 \mathrm{~d}$

$35.3 \mathrm{~d}$

$37.3 \mathrm{~d}$

$31.5 \mathrm{~d}$

$39.6 \mathrm{~d}$

$38.4 \mathrm{~d}$

40.8

35. a

$01: 02: 42$

$01: 08: 57$

$01: 06: 28$

19$$
51
$$ 
Table 5.--Distant earthquakes--Continued

September 16, 1963

U PEZ eR 20:28:10
C\&GS card 76-63:
20:05:21.9
13.4 $5 ., 166.5^{\circ} \mathrm{E}$.
Santa Cruz Is land.s
h about $28 \mathrm{~km}$
Magnitude 5.0 (CGS).

Sept. 17

$\begin{array}{lllc}\mathrm{D} & \mathrm{Z} & \mathrm{eP} & 06: 07: 08.0 \mathrm{c} \\ \mathrm{Pa} & \mathrm{Z} & \mathrm{eP} & 08.5 \mathrm{c} \\ \mathrm{U} & \mathrm{PEZ} & \mathrm{eP} & 06: 31: 52 \\ \mathrm{M} & \mathrm{Z} & \mathrm{T} \max & 07: 36: 04 \\ \mathrm{~A} & \mathrm{Z} & T \max & 34 \\ \mathrm{D} & \mathrm{Z} & T \max & 36 \\ \mathrm{MP} & \mathrm{Z} & T \max & 16 \\ \mathrm{U} & \mathrm{Z} & T \max & 22 \\ \mathrm{~Pa} & \mathrm{Z} & \mathrm{T} \max & 07: 35: 43 \\ \mathrm{Na} & \mathrm{Z} & T \max & 07: 36: 23\end{array}$

C\&GS card 75-63:

$05: 54: 33.7$

$10.6^{\circ}$ S. $78.2^{\circ} \mathrm{W}$.

Central Peru

$\mathrm{h}$ about $61 \mathrm{~km}$

Magnitude 6.75 (Pas)

5.5 (CGS).
6.0 (HVO).

Sept. 17

\begin{tabular}{|c|c|c|c|}
\hline M & $\mathrm{Z}$ & iP & $19: 28: 54.2$ \\
\hline A & Z & $\mathrm{eP}$ & 53.4 \\
\hline D & Z & $e P$ & 53.1 \\
\hline $\mathrm{U}$ & Z & iP & 54.0 \\
\hline $\mathrm{Pa}$ & Z & iP & 54.6 \\
\hline $\mathrm{Na}$ & Z & iP & 51.5 \\
\hline $\mathrm{Hi}$ & Z & iP & 54.9 \\
\hline $\mathrm{Ha}$ & $\mathrm{Z}$ & iP & 56.5 \\
\hline U & PEZ & iP & 54 \\
\hline U & PEZ & iS & $19: 36: 00$ \\
\hline U & PEZ & iR & $19: 42: 09$ \\
\hline $\mathrm{U}$ & PEN & iG & $19: 39: 47$ \\
\hline M & Z & $T \max$ & $20: 20: 44$ \\
\hline U & Z & $T \max$ & 23 \\
\hline
\end{tabular}

Sept. 17--Continued

C\&EGS card 76-63:

19:20:08.2

10. $1^{\circ} \mathrm{S} ., 165.3^{\circ} \mathrm{E}$.

Santa Cruz Islands

Felt: Eastem Solomon Islands $h$ about $17 \mathrm{~km}$

Magnitude 7.25 (Pas)

7.5 (Brk)

7 (Pal)

6.1 (CGS)

7.5 (HVO)。

Sept. 22

$M \quad Z \quad$ IP $\quad 02: 55: 57.4 \mathrm{c}$

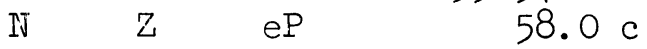

C\&GS card 76-53:

$02: 49: 03.4$

$52.5^{\circ}$ IN., $174.9^{\circ} \mathrm{W}$.

Andreanof Islands, Aleutian

Is lands.

h about $105 \mathrm{~km}$

Magnitude 4.8 (CGS).

Sept. 22

$\begin{array}{lllrl}\mathrm{M} & \mathrm{Z} & \mathrm{eP} & 03: 05: 01.7 \mathrm{~d} \\ \mathrm{~A} & \mathrm{Z} & \mathrm{eP} & 00.6 \mathrm{~d} \\ \mathrm{D} & \mathrm{Z} & \mathrm{eP} & 00.4 \mathrm{~d} \\ \mathrm{~Pa} & \mathrm{Z} & \mathrm{eP} & 02.7 \mathrm{~d} \\ \mathrm{Hi} & \mathrm{Z} & \mathrm{eP} & 03.3 \mathrm{~d} \\ \mathrm{Ha} & \mathrm{Z} & \mathrm{eP} & 05.3 \mathrm{c} \\ \mathrm{U} & \mathrm{PEZ} & \mathrm{iP} & 01 & \mathrm{~d} \\ \mathrm{U} & \mathrm{PEZ} & \mathrm{eR} & 03: 18: 10 & \\ \mathrm{U} & \mathrm{PEN} & \text { iS } & 03: 12: 12 & \end{array}$

C\&GS card 82-63:

$02: 56: 24.3$

$19.3^{\circ} \mathrm{S} ., 175.9^{\circ} \mathrm{E}$.

Fiji Islands region

h about $28 \mathrm{~km}$

Magnitude 5.8 (CGS).

Sept. 22

$\begin{array}{lllr}M & Z & \text { iP } & 19: 30: 33.7 \mathrm{c} \\ \mathrm{A} & \mathrm{Z} & \text { iP } & 33.4 \mathrm{c} \\ \text { IN } & \text { Z } & \text { iP } & 33.6 \mathrm{c}\end{array}$


Table 5.--Distant earthquakes--Continued

September 22, 1963--Continuea

$\begin{array}{llll}M P & Z & i P & 33.3 \mathrm{c} \\ \mathrm{Pa} & \mathrm{Z} & \mathrm{eP} & 34.6 \mathrm{~d} \\ \mathrm{Na} & \mathrm{Z} & \mathrm{eP} & 30.1 \mathrm{~d} \\ \mathrm{Ka} & \mathrm{Z} & \mathrm{eP} & 35.9 \mathrm{c} \\ \mathrm{Hi} & \mathrm{Z} & \mathrm{eP} & 37.3 \mathrm{c}\end{array}$

C\&GS card 76-63:

$19: 21: 57.1$

$19.2^{\circ} \mathrm{S}, 175.9^{\circ} \mathrm{E}$.

Tonga Is larids region

h about $24 \mathrm{~km}$.

Sept. 23

M Z e.P 1.7:09:51.0 c

C\&GS card 76-63:

$17: 02: 36.6$

$51.3^{\circ}$ N., $179.0^{\circ} \mathrm{W}$.

Andreanof Is lands, Aleutian

Is lands.

$\mathrm{h}$ about $33 \mathrm{~km}$

Magnitude 5.2 (CGS).

Sept. 24

\begin{tabular}{|c|c|c|c|}
\hline M & Z & iP & $16: 42: 28.8$ \\
\hline A & Z & $j P$ & 28.2 \\
\hline $\mathrm{D}$ & $\mathrm{Z}$ & iP & 28.3 \\
\hline $\mathrm{N}$ & Z & $2 . T$ & 28.3 \\
\hline MP & Z & $i^{T}$ & 27.4 \\
\hline $\mathrm{Pa}$ & Z & ¿EF & 29.0 \\
\hline $\mathrm{Na}$ & Z & $\therefore F$ & 2.9 .5 \\
\hline $\mathrm{Ka}$ & Z & i. $\mathrm{P}$ & 31.6 \\
\hline $\mathrm{Ha}$ & Z & jp & 32.5 \\
\hline $\mathrm{U}$ & PEZ & ip & 8 \\
\hline U & PEZ & $\therefore \mathrm{pP}$ & $16: 43: 04$ \\
\hline $\mathrm{U}$ & PEZ & ¿PP & $16: 45: 42$ \\
\hline $\mathrm{U}$ & $\mathrm{PE} \angle$ & ePs & $16: 53: 37$ \\
\hline U & PEZ & eSs & $16: 58: 13$ \\
\hline U & PEZ & $\mathrm{eP}$ & $1.7: 07: 28$ \\
\hline U & PEN & iS & $.16: 52: 38$ \\
\hline U & PEN & ESSS & $17: 01: 52$ \\
\hline U & PEN & $i C_{i}$ & $17: 04: 20$ \\
\hline U & $\mathrm{PEE}$ & eis & $15: 53: 1.8$ \\
\hline U & $\mathrm{PEE}$ & $\begin{array}{l}\text { iss.PS/ } \\
\text { sijp }\end{array}$ & $16: 54: 09$ \\
\hline
\end{tabular}

Sept. 24--Continued

C\&GS card 76-63:

$16: 30: 16.0$

$10.6^{\circ} \mathrm{S} ., 78.0^{\circ} \mathrm{W}$.

Near coast of Peru

$\mathrm{h}$ about $80 \mathrm{~km}$

Magnitude 7 (Pas)

6.5 (Brk)

6.0 (CGS)

Sept. 25

$$
6.6 \text { (HVO). }
$$

M Z e $\quad$ I4:59:06.3 d

C\&CSS card 77-63:

$14: 50: 18.2$

10. $1^{\circ}$ S., $164.5^{\circ} \mathrm{E}$.

Solomon Islands region

$\mathrm{h}$ about $33 \mathrm{~km}$

Magnituảe 5.1 (CGS).

Sept. 26

$\begin{array}{llll}\mathrm{Pa} & Z_{1} & \text { Tinax } & 05: 06: 18 \\ \mathrm{Ha} & \mathrm{Z} & \text { Tmax } & 05: 05: 01\end{array}$

C\&GS card 77-63:

$04: 20: 21.5$

$56.5^{\circ}$ N., $153.4^{\circ} \mathrm{W}$.

Kodiak Is land region

$\mathrm{h}$ about $33 \mathrm{~km}$

Magnitude 4.8 (CGS).

Sept. 26

$\begin{array}{llll}M & \text { Z } & \text { eP } & 05: 35: 00.4 c \\ U & \text { PEZ } & \text { eS } & 05: 40: 42 \\ U & \text { PEZ } & \text { eR } & 05: 44: 14 \\ \text { U } & \text { PEE } & \text { eL } & 05: 42: 28\end{array}$

C\&GS card 76-63:

$05: 28: 07.3$

$50.4^{\circ} \mathrm{N}$. , $176.9^{\circ} \mathrm{W}$.

Andreanof Islands, Aleutian

Islands.

h about $33 \mathrm{~km}$

Magnitude 5.3 (CGS). 


\section{September 26, 1963}

$\begin{array}{llll}\text { M } & \text { Z } & T \text { max } & 07: 26: 51 \\ \text { A } & \text { Z } & T \text { max } & 07: 27: 16 \\ \text { D } & \text { Z } & \text { Tmax } & 07: 27: 20 \\ \text { MP } & \text { Z } & \text { Tmax } & 07: 27: 20 \\ \text { U } & \text { Z } & \text { Tmax } & 07: 27: 09 \\ \mathrm{~Pa} & \text { Z } & \text { Tmax } & 07: 27: 04 \\ \mathrm{Ha} & \mathrm{Z} & T \max & 07: 25: 31\end{array}$

C\&GS card 77-63:

$06: 40: 43.5$

$56.6^{\circ}$ N., $153.2^{\circ}$ W.

Kodiak Island region

$\mathrm{h}$ about $33 \mathrm{~km}$

Magnitude 4.8 (CGS).

Sept. 27

$\begin{array}{llll}\text { U } & \text { PEN } & \text { eS } & 11: 41: 24 \\ U & \text { PEN } & \text { eG } & 11: 44: 49 \\ U & \text { PEZ } & \text { eR } & 11: 46: 53\end{array}$

C\&GS card 79-63:

$11: 25: 53.6$

$17.2^{\circ}$ S., $174.7^{\circ} \mathrm{E}$.

Fiji Islands region

$\mathrm{h}$ about $33 \mathrm{~km}$

Magnitude 5.0 (CGS).

\section{Sept. 28}

$\begin{array}{lllr}M & Z & \text { iP } & 07: 07: 09.4 \mathrm{~d} \\ \mathrm{~A} & \mathrm{Z} & \text { iP } & 08.9 \mathrm{~d} \\ \mathrm{D} & \mathrm{Z} & \text { iP } & 08.0 \mathrm{~d} \\ \mathrm{MP} & \mathrm{Z} & \text { iP } & 08.9 \mathrm{~d} \\ \mathrm{U} & \mathrm{Z} & \text { iP } & 09.2 \mathrm{~d} \\ \mathrm{Hi} & \mathrm{Z} & \mathrm{eP} & 10.4 \mathrm{~d} \\ \mathrm{Na} & \mathrm{Z} & \mathrm{eP} & 06.1 \mathrm{~d} \\ \mathrm{Ka} & \mathrm{Z} & \text { iP } & 11.6 \mathrm{~d}\end{array}$

C\&GS card 79-63:

$06: 58: 12.7$

$31.5^{\circ} \mathrm{S}, 179.6^{\circ} \mathrm{E}$.

Kermadec Isla nds

$\mathrm{h}$ about $457 \mathrm{~km}$

Magnitude 5.0 (CGS). 
During the quarter "felt reports" were either phoned or mailed in by the following people to whom we wish to express our gratitude for these and other instances of cooperation.

North Hawaii

Mrs. Lindsey

Mrs. Ecklund

Mrs. Van Gorder

Dr. Heather

$\mathrm{Mr}$. Hea

Mrs. Calles

\section{$\underline{\text { Kau }}$}

Mrs. Billings

Mrs. Paiva

Mr. Meinecke

\section{Hilo region}

Mrs. Shoemaker

Mrs. Ingledue

Mrs. Shaffer

Mr. Baldwin

Mrs. Baldwin

Mr. Donahoe

$\mathrm{Mr}$. Ho

Mr. Wessel

Mr. Potlock

Mr. Usagawa

Mr. Kumuhaki

Mr. Reeves

Mr. Guerimo

Puna

Mrs. Isbell

Mrs. Kimura

Miss Takemoto

\section{Kona coast}

Mr. Johnston

Mr. Glass

Mrs. Fujino

Mr. Sutherland

Miss Greenwell

Miss Wallace

Mr. Apple

Mrs. Apple

$\mathrm{Mr}$. Ladd.

\section{$\underline{\text { Kilauea summit area }}$}

Mrs. Mist

$\mathrm{Mr}$. Koyanagi

Mrs. Forbes

Dr. Wentworth

Dr. Moore

Mr. Francis

Mr. Young

$\mathrm{Mr}$. Yamamoto

Miss English

Mr. Shipman

Mr. Correa

Mr. Cuskelly

Mrs. Hansen

Central Hawaii

Kulani Honor Camp 
UNITED STATES

DEPARTMENT OF THE INTERIOR

GEOLOGICAL SURVEY

\section{HAWAIIAN VOLCANO OBSERVATORY \\ SUMMARY 32 \\ October, November, and December 1963}

By

Arnold T. Okamura, Robert Y. Koyanagi, Willie T. Kinoshita,

J. G. Moore, D. L. Peck, and Howard A. Powers

Observatory staff

Geology

J. G. Moore (Scientist-in-charge)

Geochemistry

D. L. Peck

C. K. Wentworth (retired Dec. 1963)

R. T. Okamura

Geophysics

\section{Support}

W. T. Kinoshita

E. T. Endo

G. Kojima

J. C. Forbes

R. Y. Koyanagi

W. H. Francis

H. L. Krivoy

B. J. Loucks

A. T. Okamura

A. S. Ryall (left 11-27-63)

N. Sherrill (left 11-19-63)

\section{Visiting Japanese scientists}

(Cooperative program ended Dec. 31, 1963)

T. Minakami (Group leader) C. Kurihara

D. Shimozuru M. Kurihara

K. Kamo

S. Hiraga 


\section{Contents}

Page

Chronological summary-1

Tilting of the ground around Kilauea caldera-1..- 4

Seismic summary-1-0 8

Persons or agencies reporting felt earthquakes during the quarter---- 34

Publications of special interest and HVO numbered contributions, 1963

\section{Illustrations}

Figure 1. Map of the island of Hawaii showing seismograph stations and localities mentioned in the text....... 3

2. Tilting of the ground around Kilauea caldera-.-...-..- 7

Tables

Table 1. Tilt coordinates at Uwekahuna Vault-1... 5

2. Tilt coordinates and changes at bases around Kilauea

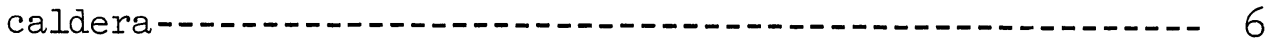

3. Numbers of earthquakes and minutes of tremor recorded on seismographs around Kilauea caldera-............. 9

4. Local earthquakes recorded by seismographs of the U.S. Geological Survey-_... 14

5. Distant earthquakes 
A flank eruption lasting less than 30 hours occurred on the central part of the east rift zone of Kilauea Volcano (fig. A) on October 5 and 6, 1963, just 43 days after the last previous eruption in Alae Crater.

The eruption was preceded by a marked deflation of the summit of Kilauea, which began at $03^{\mathrm{h}} 06^{\mathrm{m}}$ October 5 , as indicated by a deflection of the long-period seismographs at Uwekahuna. Ten minutes later seismographs at the summit and upper east rift zone began to record strong harmonic tremor and local shallow earthquakes. Harmonic tremor increased markedly after $05^{\mathrm{h}} 25^{\mathrm{m}}$, which may have marked the beginning of the eruption in Napau Crater.

A pilot who flew over Napau Crater at $06^{\mathrm{h}} 05^{\mathrm{m}}$ on a flight to Naalehu observed lava fountaining from a fissure extending across the floor of the crater. When an Observatory party arrived at Makaopuhi Crater at $06^{\mathrm{h}} 08^{\mathrm{m}}$ they observed a billowing fume cloud and heard a faint roar to the northeast. At $08 \mathrm{~h}_{50} \mathrm{~m}$ when they reached the southwest rim of Napau Crater an active line of lava fountains up to 50 feet high extended across the floor of the pit crater slightly north of the 1840 spatter ridge, and 80 percent of the floor of the crater was flooded with 20 feet of lava. Spatter and a small pad of lava across the trail northwest of Napau was still fuming when first seen at $09^{\mathrm{h}} 05^{\mathrm{m}}$.

Beginning at $09^{h} 30^{m}$ regular aerial observation of the eruption was begun. At that time a line of vents extended from the west side of Napau Crater 4 miles to the northeast along the rift zone. At about $14^{\mathrm{h}} \mathrm{OO}^{\mathrm{m}}$ a quarter-mile line of 150-foot-high fountains broke out farther down the rift zone near Kalalua Crater. These fed two lava flows that were moving side by side down the rift zone. By 20 h $30 \mathrm{~m}$ the flows had reached slightly more than a mile from the source fountains and were moving at less than $1 / 4$ mile per hour; by $04^{\mathrm{h}} 00^{\mathrm{m}}$ the next morning the glow of fountains had ceased and the flows, now $11 / 2$ miles long, had stopped. The final outbreak of the eruption occurred at $0 \mathrm{gh}_{40 \mathrm{~m}}$ on a fissure about 3 miles northeast of Napau Crater. Lava fountained briefly to heights of 25 feet, but by $09 \mathrm{gh}_{50 \mathrm{~m}}$ the vent spattered weakly and presumably it soon died.

The site of the eruption, an inaccessible area of rain forest, severely hampered observations both during and after the eruption. However, the upper half of the area was examined on foot in October and December, and the lower part was sampled and mapped by helicopter in January. These studies, together with aerial photographs of the area taken by the Navy in November, show that an almost continuous line of active vents extended $7-3 / 4$ miles northeastward along the rift zone from a point $1 / 3 \mathrm{mile}$ west of Napau Crater. The vents are on the extreme north side of the rift zone, and are arranged in a right offset en echelon pattern. In addition to the small areas of spatter the eruption produced a shallow lava lake in Napau Crater and two major flows: a mile-long flow 2 to 3 miles northeast of Napau, and a double flow 
( $2 \frac{1}{2}$ miles long and as much as $\frac{1}{2}$ mile wide) north of Kalalua Crater. The flows contain from less than 1 percent to 15 percent olivine, probably cover about $1-1 / 3$ square miles, and contain about 10 million cubic yards of lava. Occupation of the network of long-base tilt stations around the summit of Kilauea showed a marked deflation of the summit area between August 6 and October 10, and the short-base tilt instruments at Uwekahuna indicated that the detumescence associated with the October eruption was equivalent to the removal of approximately 30 million cubic yards of magma from the summit reservoir.

J. G. M. and D. L. P.

During the eruption, readings of the short-base tiltmeter (table 1) showed strong collapse along the direction $\mathrm{E} .28^{\circ} \mathrm{S}$, and for a week following the eruption showed moderate collapse to the south. For about a month, from October 15 to November 15, the tilt indicated slow tumescence along the direction $\mathrm{W} .28^{\circ} \mathrm{N}$. This was accomparied by a sporadic, moderate increase in the daily number of local caldera quakes. From mid-November to the year-end, tilt recovered slightly in a due west direction. Total recovery of tilt from the end of the eruption to the end of the year was less than $1 / 2$ to the west and $1 / 5$ to the north of that lost in the collapse. The daily number of small local quakes again showed sporadic and moderate increase during the last six weeks, and there was a slight peak of 30 deep small quakes on December 26.

Mid-October to early November was a period of high earthquake activity along the Kaoiki fault system. On October 23 a Kaoiki quake of magnitude 5.3 occurred and was followed by several hundred smaller shocks. Several of these were felt.

Two distant earthquakes originating in the Kurile Islands were large enough to alert coastal residents of Hawaii of possible sea wave activity. These occurred on October 12 and 20 (H.S.T.) with magnitudes of 8.1 and 7.6 , respectively. No damaging sea wave occurred in Hawaii. 


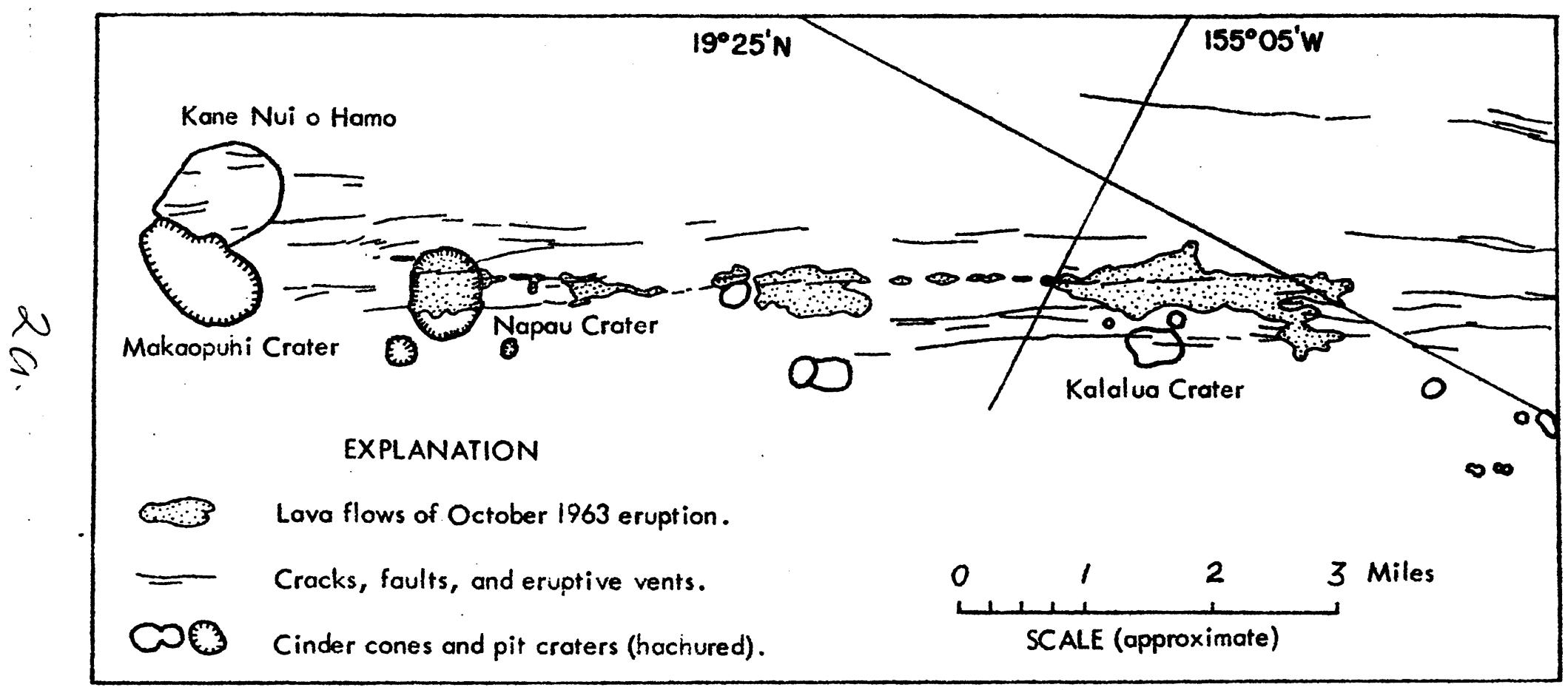

Figure A. Preliminary map showing area of lava erupted october 5-6, 1963. (East Rift Zone) 


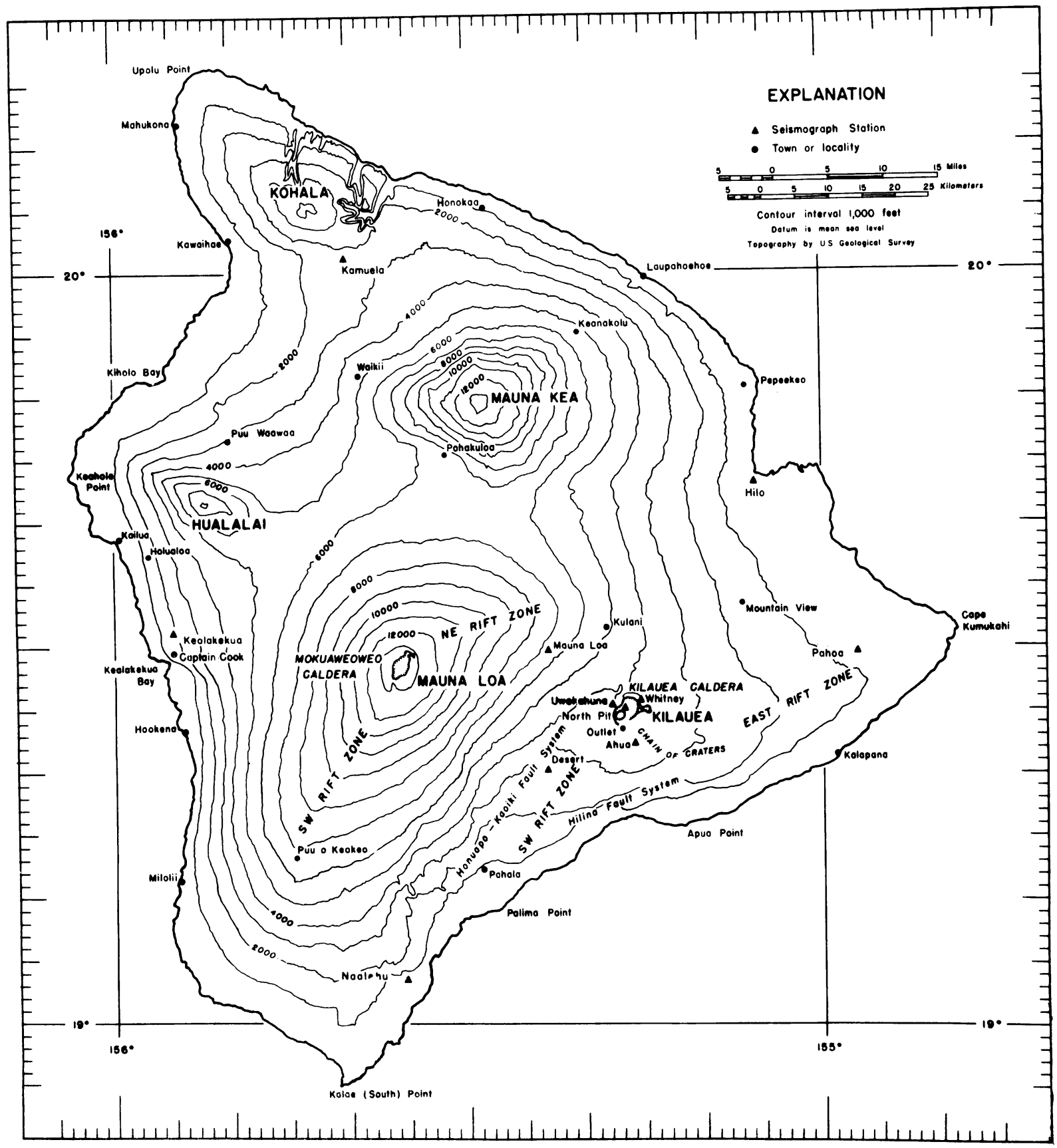

Figure 1.--Map of the island of Hawaii showing seismograph stations operated by the Geological Survey and localities mentioned in the text. Epicenters of local earthquakes are given in terms of geographic coordinates, which are indicated at the edges of the map. 
Tilting of the ground around Kilauea caldera.--Tilting of the ground around the summit of Kilauea is monitored daily by a short-base water-tube tiltmeter in Uwekahuna Vault, and at irregular intervals it is measured on a regional scale by means of a network of field tilt-bases and a portable water-tube tiltmeter. The attitude of the ground surface at each tilt base is reported in terms of north-south and east-west tilt coordinates. Both coordinates at each station were arbitrarily set equal to 500 when measurements at that station were begun. Increasing tilt coordinates correspond to northward and eastward tilting of the earth's surface, i.e., to a relative subsidence toward the north and east. A one unit-change in coordinate corresponds to a tilting of 1 microradian ( $1 \mathrm{~mm}$ per $\mathrm{km}$ ) in the direction indicated. 
Table 1. - Tilt coordinates at Uwekahuna Vault, October, November, and December, 1963

\begin{tabular}{rll||ccc}
\hline Date & N-S & E-W & Date & N $-S$ & E-W \\
\hline Oct. 6 & 480 & 516 & Dec. 1 & 467 & 511 \\
13 & 461 & 538 & 8 & 467 & 510 \\
20 & 461 & 535 & 15 & 467 & 510 \\
27 & 462 & 530 & 22 & 467 & 506 \\
Nov. 3 & 465 & 522 & 29 & 468 & 505 \\
10 & 466 & 519 & & & \\
17 & 467 & 514 & & & \\
24 & 467 & 513 & & & \\
\hline
\end{tabular}


Table 2. - Tilt coordinates and changes at bases around Kilauea caldera (see fig. 2)

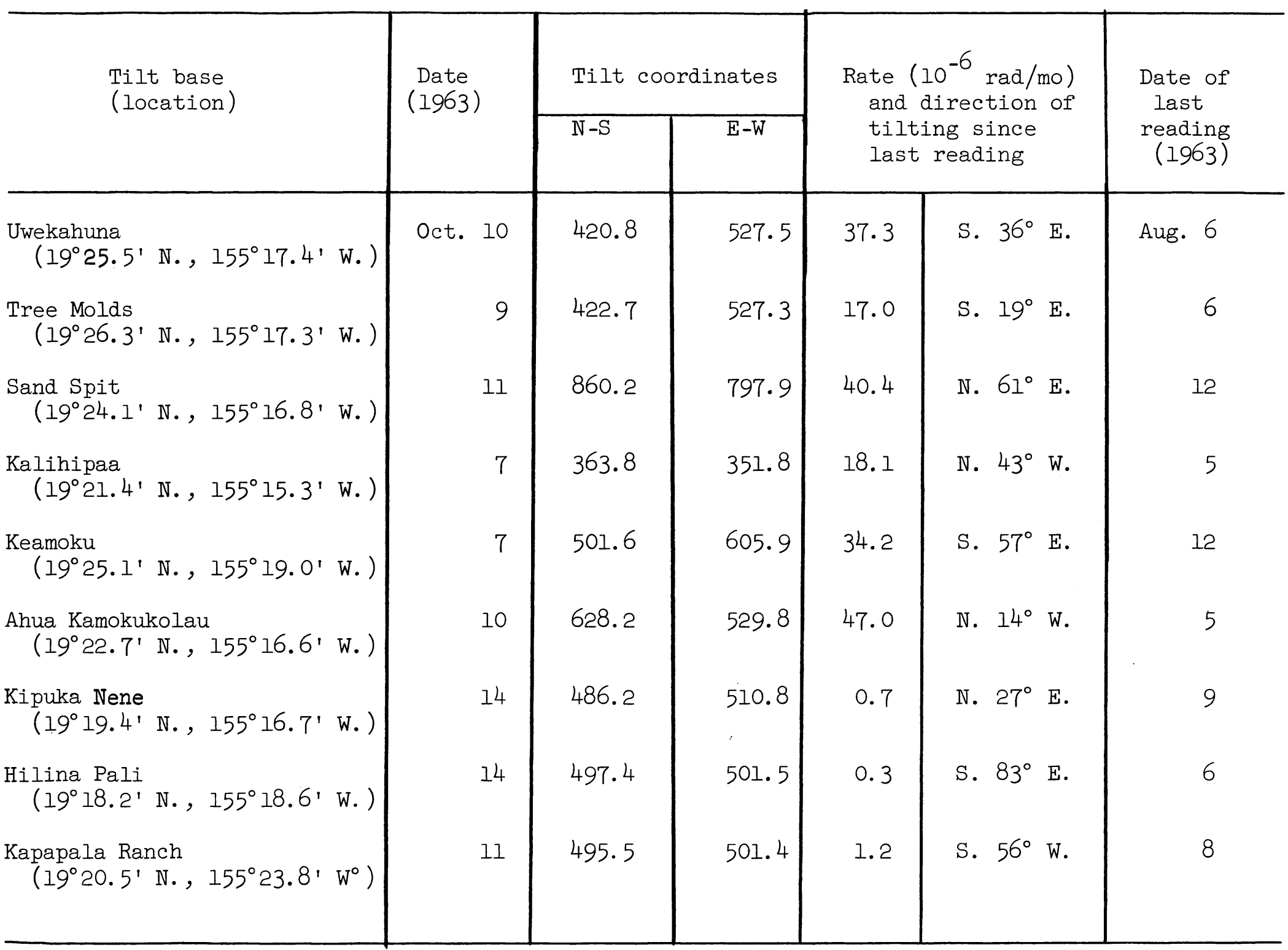




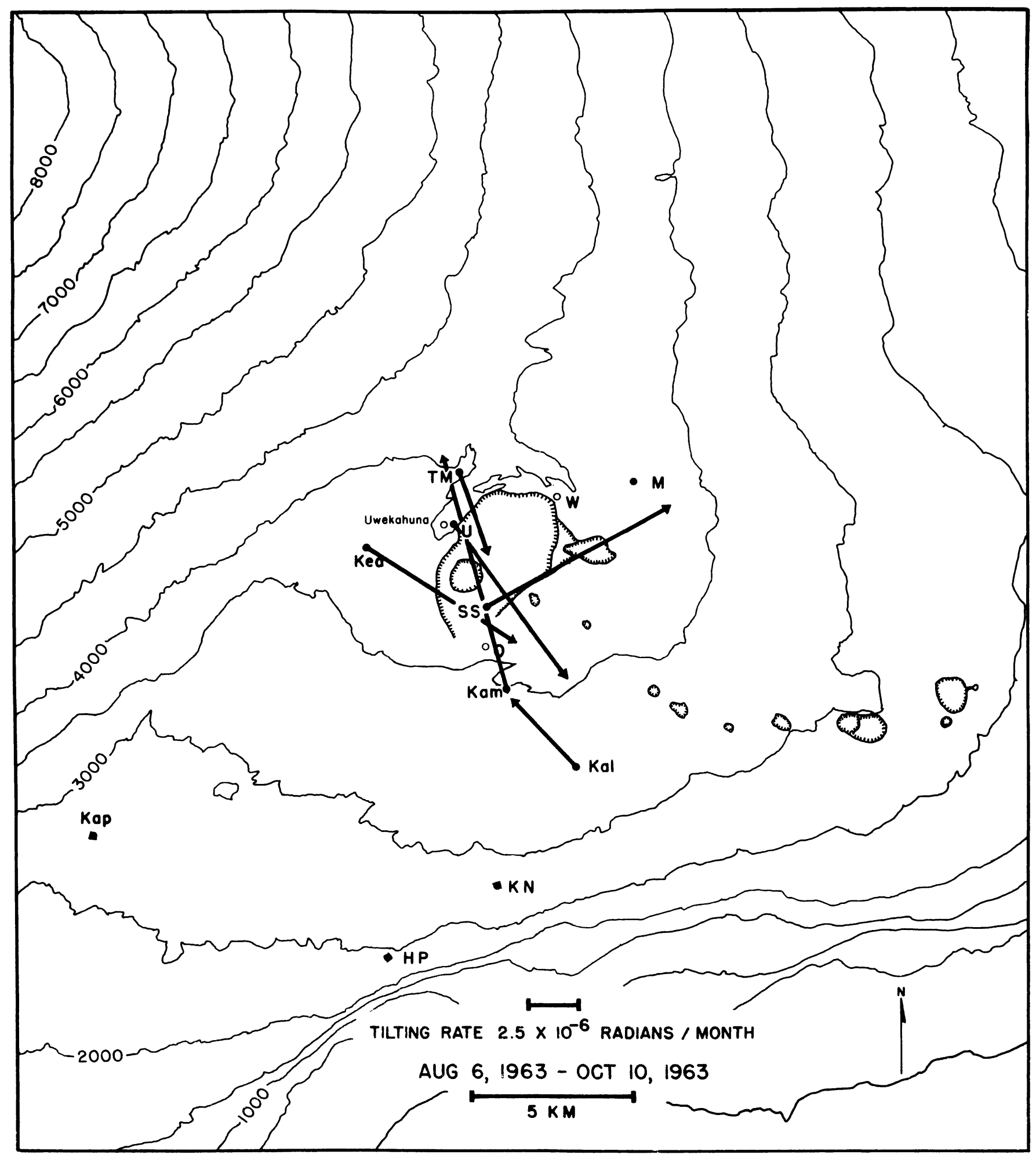

Figure 2.--Tilting of the ground around Kilauea caldera, August 6 to October 10, 1963. The vector depicting tilting at a given tilt base points in the direction of maximum relative subsidence and has a length proportional to the rate of tilting during the measurement interval. Closed circles represent field tilt bases; open circles, short-base water-tube tiltmeters. 
Seismic summary. --Events recorded by the U.S. Geological Survey seismograph network in Hawaii fall into two categories: Local earthquakes and tremor originating in the region of the Hawaiian Islands, usually within $100 \mathrm{~km}$ of at least one seismograph, and distant earthquakes originating more than $3,000 \mathrm{~km}$ from Hawaii. As an index of seismic activity at Hawaiian volcanoes, daily counts of earthquakes and minutes of tremor recorded by seismographs in Hawaii are listed in table 3. The earthquakes are separated into groups on the basis of region of origin as determined by analysis of records obtained daily at the Observatory ( $U, M, A, D, N, M P$ ). Earthquakes of magnitude 2.5 or greater are generally sufficiently well recorded to be located with greater precision; they are listed individually in table 4. Data on identifiable phases from distant earthquakes are listed in table 5.

Locations of the seismograph stations are shown in Figure 1 , and essential data on the stations were given in Summaries 25 and 29. 
Table 3. - -Numbers of earthquakes and minutes of tremor recorded on seismographs

\section{$\mathrm{U}, \mathrm{M}, \mathrm{A}, \mathrm{D}, \mathrm{N}, \mathrm{WP}$, and $\mathrm{MP}$ around Kilauea caldera}

Tremor is separated into three categories: deep, intermediate, and shallow, on the basis of relative amplitudes on seismographs in the summit region. Unless otherwise stated, tremor is presumed to be associated with movement of magma within the central complex of Kilauea.

Earthquake categories are: Halemaumau rock slides, which are detected by the characteristic record they produce on the North Pit seismograph; shallow earthquakes in the Kilauea caldera region; shallow earthquakes along the SW. rift zone of Kilauea and the adjacent portion of the Kaoiki fault system; earthquakes along the eastern half of Kilauea's east rift zone--detected largely on the Pahoa short-period vertical; earthquakes from a source about $30 \mathrm{~km}$ beneath Halemaumau; earthquakes from the upper east rift zone ahd the adjacent fault systems of Kilauea's south flank (these are usually first arrivals at the Ahua meter or at the new experimental geophone near Makaopuhi Crater (MP)); and earthquakes from other regions: Kona, Mauna Kea, etc.

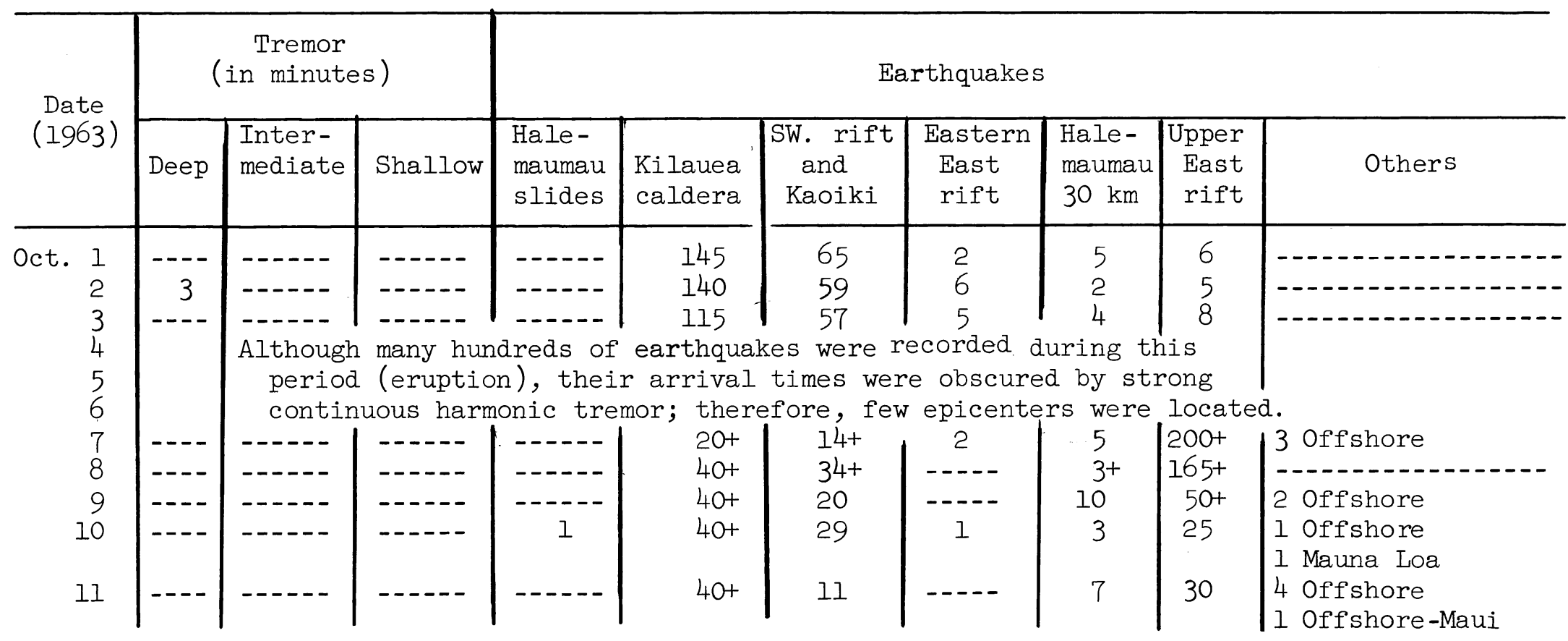


Table 3.-Numbers of earthquakes and minutes of tremor recorded on seismographs

$\mathrm{U}, \mathrm{M}, \mathrm{A}, \mathrm{D}, \mathrm{N}, \mathrm{WP}$, and $\mathrm{MP}$ around Kilauea caldera--Continued

\begin{tabular}{|c|c|c|c|c|c|c|c|c|c|c|}
\hline \multirow{2}{*}{$\begin{array}{l}\text { Date } \\
(1963)\end{array}$} & \multicolumn{3}{|c|}{$\begin{array}{c}\text { Tremor } \\
\text { (in minutes) }\end{array}$} & \multicolumn{7}{|c|}{ Earthquakes } \\
\hline & Deep & $\begin{array}{l}\text { Inter- } \\
\text { mediate }\end{array}$ & Shallow & $\begin{array}{l}\text { Hale- } \\
\text { maumau } \\
\text { slides }\end{array}$ & $\begin{array}{l}\text { Kilauea } \\
\text { caldera }\end{array}$ & $\begin{array}{l}\text { SW. rift } \\
\text { and } \\
\text { Kaoiki }\end{array}$ & $\begin{array}{c}\text { Eastern } \\
\text { East } \\
\text { rift }\end{array}$ & $\begin{array}{l}\text { Hale- } \\
\text { maumau } \\
30 \mathrm{~km}\end{array}$ & $\begin{array}{l}\text { Upper } \\
\text { East } \\
\text { rift }\end{array}$ & Others \\
\hline $\begin{array}{ll}\text { Oct. } & 12 \\
13 \\
\\
14 \\
\\
15 \\
16 \\
17 \\
18 \\
19 \\
20 \\
21 \\
22 \\
\\
23 \\
24 \\
25 \\
26 \\
27 \\
28 \\
29 \\
30 \\
\\
31\end{array}$ & 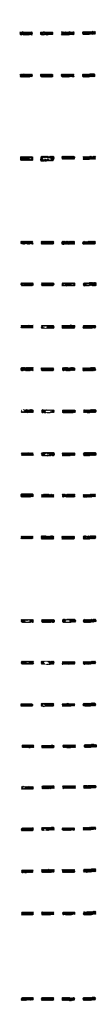 & 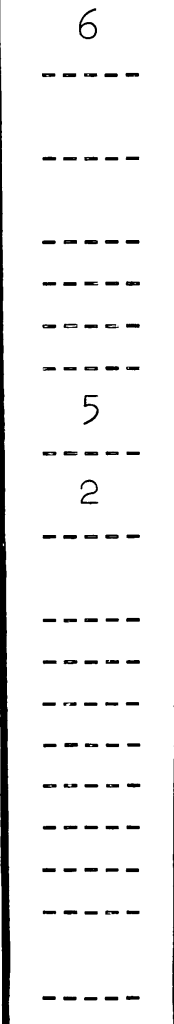 & 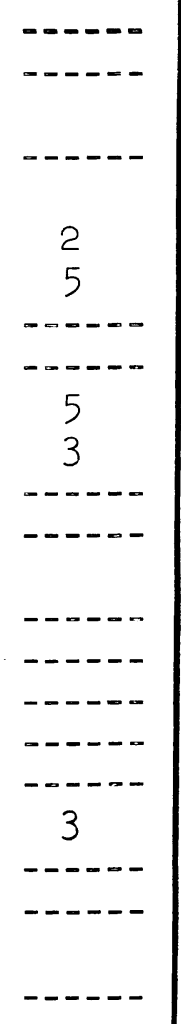 & 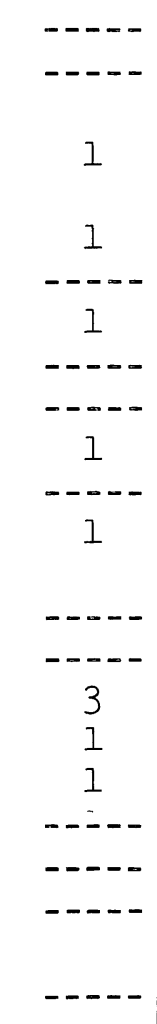 & $\begin{array}{l}25+ \\
30+ \\
40 \\
\\
15 \\
30+ \\
20 \\
17 \\
25 \\
25+ \\
50+ \\
30 \\
\\
43 \\
40 \\
60 \\
35 \\
70 \\
90 \\
70 \\
80 \\
\\
80\end{array}$ & $\begin{array}{l}85+ \\
40 \\
\\
21 \\
14 \\
23 \\
21 \\
10 \\
19 \\
12 \\
14 \\
17 \\
425+ \\
76 \\
115 \\
73 \\
64 \\
40 \\
34 \\
98 \\
70\end{array}$ & 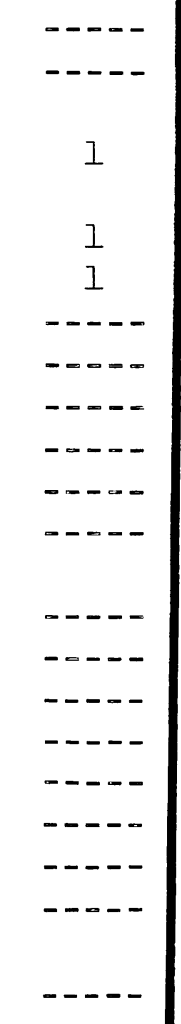 & 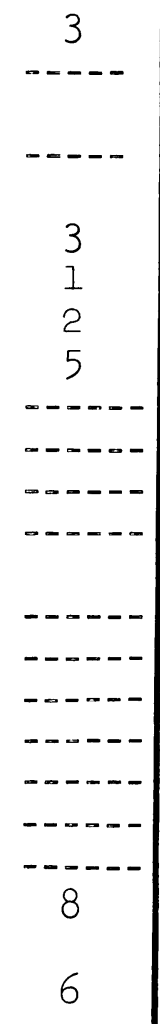 & $\begin{array}{r}10 \\
9 \\
8 \\
7 \\
7 \\
-17 \\
5 \\
4 \\
17 \\
10 \\
5 \\
4 \\
5 \\
8 \\
8 \\
4 \\
4 \\
5 \\
40 \\
26\end{array}$ & $\begin{array}{l}1 \text { Offshore } \\
1 \text { Kona } \\
1 \text { Offshore } \\
1 \text { Offshore } \\
1 \text { Kona } \\
\text { I S. Pali } \\
\text { I Offshore } \\
2 \text { Offshore } \\
1 \text { Offshore-Maui } \\
\text { I Kona } \\
-1 \text { Mauna Loa } \\
\text { I Mauna Loa } \\
\text { I Mauna Loa } \\
\text { I Offshore-Maui } \\
\text { I Offshore } \\
\text { - }\end{array}$ \\
\hline
\end{tabular}


Table 3.--Numbers of earthquakes and minutes of tremor recorded on seismographs

$\mathrm{U}, \mathrm{M}, \mathrm{A}, \mathrm{D}, \mathrm{N}, \mathrm{WP}$, and $\mathrm{MP}$ around Kilauea caldera--Continued

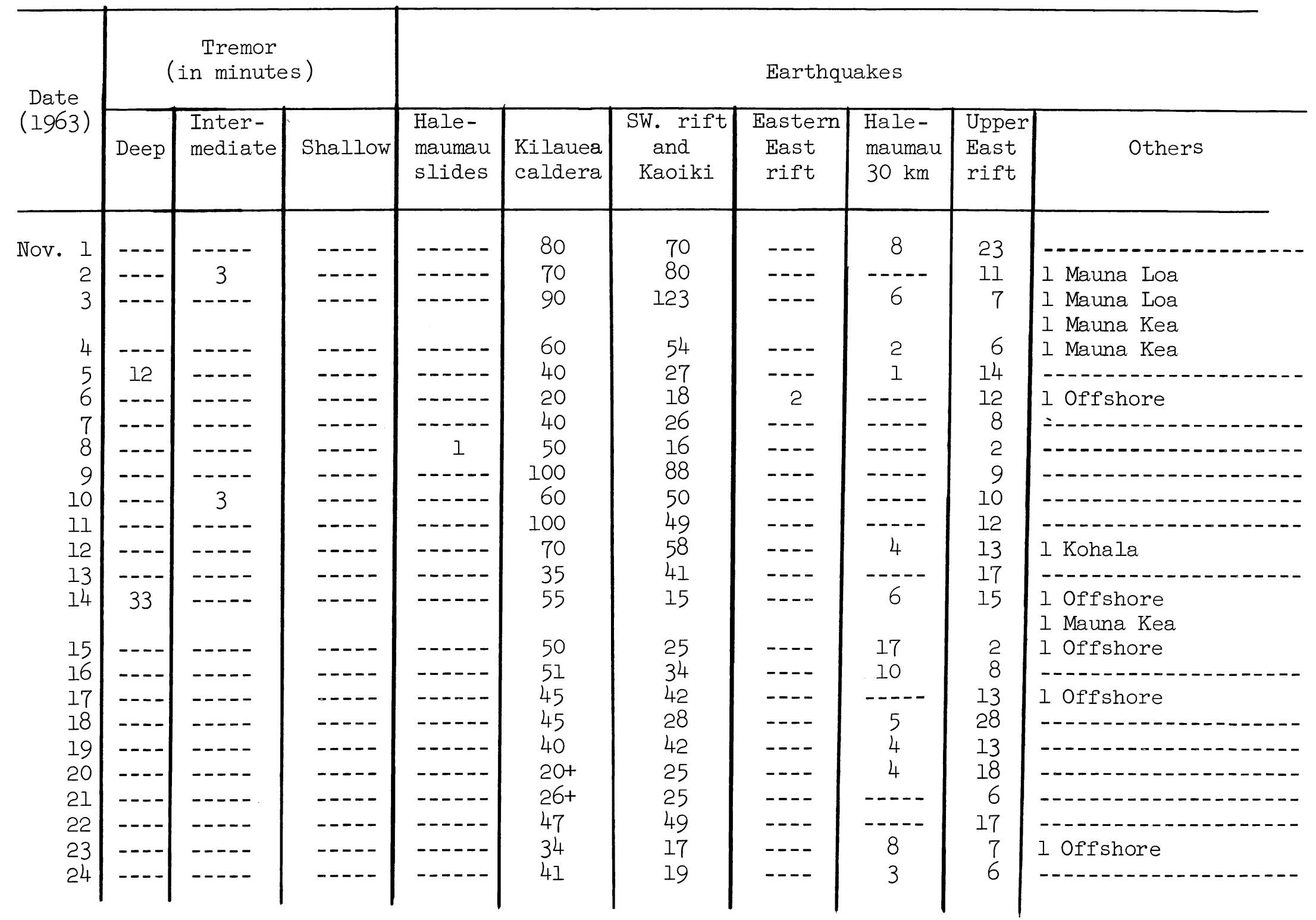


Table 3.--Numbers of earthquakes and minutes of tremor recorded on seismographs

\section{$\mathrm{U}, \mathrm{M}, \mathrm{A}, \mathrm{D}, \mathrm{N}, \mathrm{WP}$, and $\mathrm{MP}$ around Kilauea caldera--Continued}

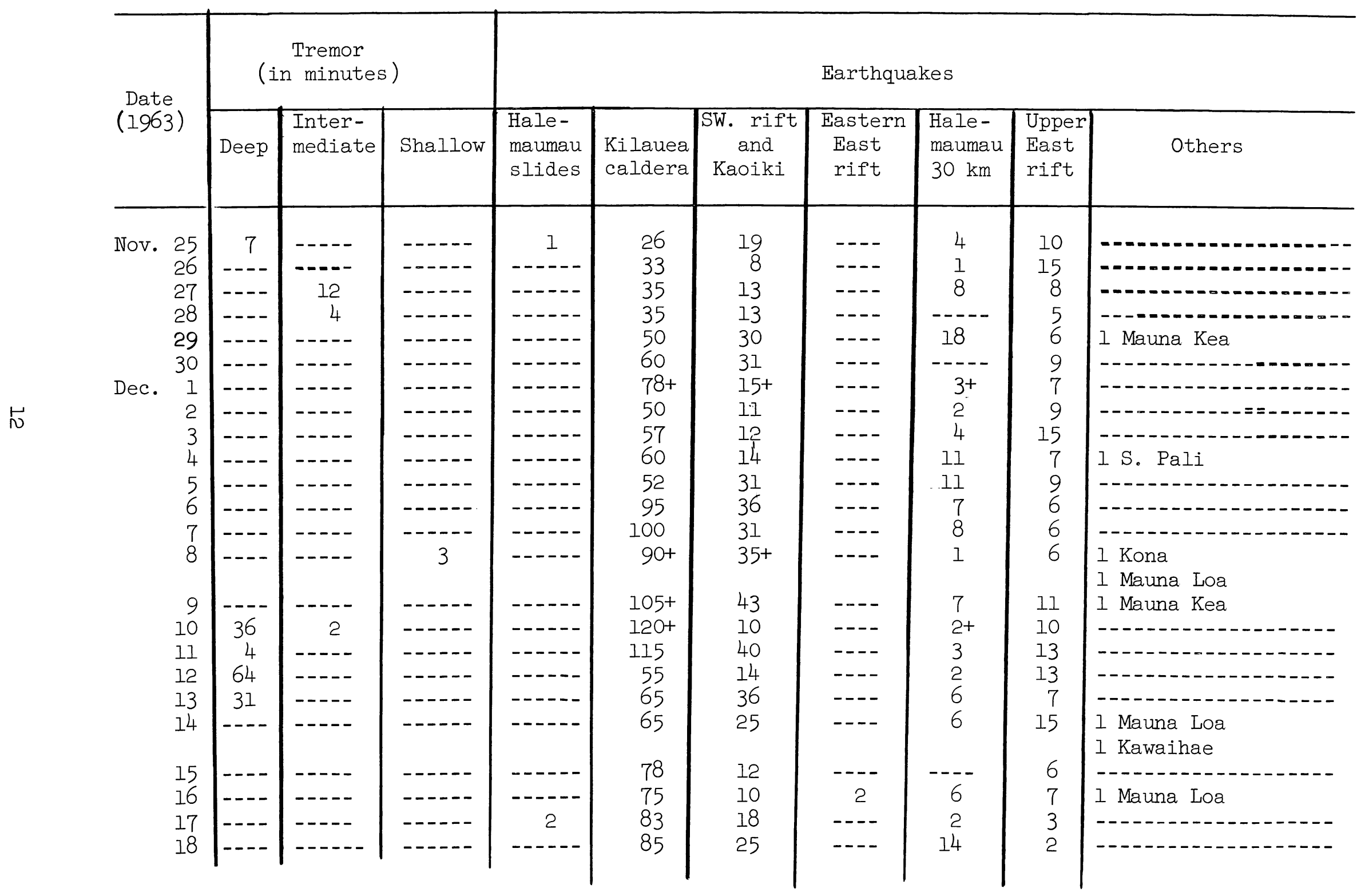


Table 3.--Numbers of earthquakes and minutes of tremor recorded on seismographs

$\mathrm{U}, \mathrm{M}, \mathrm{A}, \mathrm{D}, \mathrm{N}, \mathrm{WP}$, and $\mathrm{MP}$ around Kilauea caldera--Continued

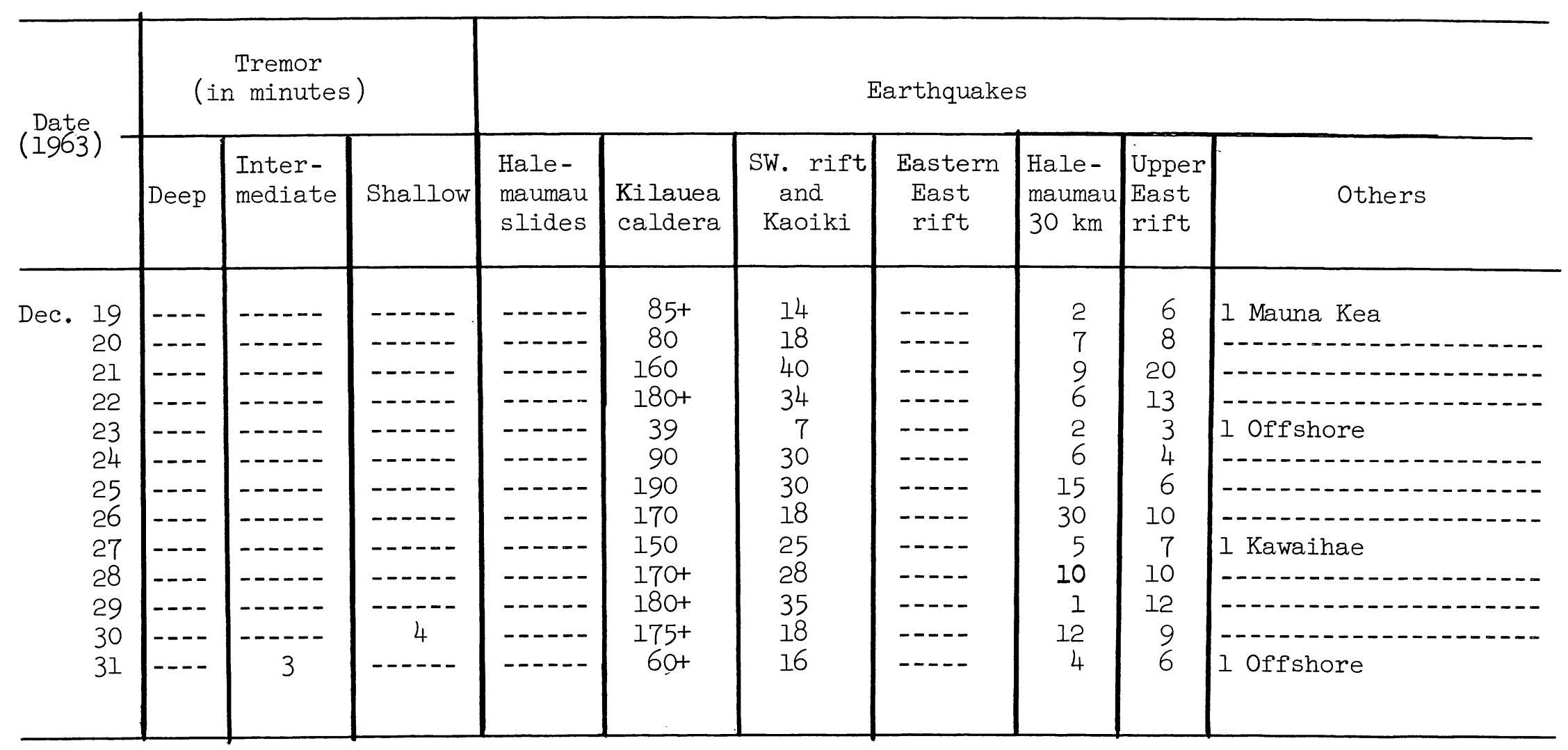


Table 4. --Local earthquakes recorded by seismographs of the U.S. Geological Survey, October, November, and December, 1963

[Entries for a given quake are: date, origin time (Hawaiian Standard Time), magnitude, depth, epicenter, and felt report. All earthquakes of magnitude 2.5 and larger, as well as many favorably located smaller ones, occurring on or near the island of Hawaii are included in the list.

In the following list, some origin times are followed only by "KM 30" and a statement of magnitude. These are all members of a continuing family of quakes noted also in other Summaries. The best mean focus for this group is beneath Halemaumau at a depth of $30 \mathrm{~km}\left(19^{\circ} 24.1^{\prime} \mathrm{N} ., 155^{\circ} 17.1^{\prime} \mathrm{W}.\right)$.

In the following list a number of quakes are described as "Upper east rift" (see Summary 28). Further statistical study of this group which occurred in the swarm periods during July 1 to 6 and August 3 to 4 gives a mean epicenter $19^{\circ} 21.5^{\prime} \mathrm{N} ., 155^{\circ} 14^{\prime} \mathrm{W}$. about $2 \mathrm{~km}$ south of Aloi Crater at near-surface depth.

In Summary 24, "Kaoiki" was introduced as a symbol for listing any of a family of quakes with mean focus $19^{\circ} 24^{\prime} \mathrm{N} ., 155^{\circ} 24^{\prime} \mathrm{W} ., \mathrm{h}=3$ to $8 \mathrm{~km}$. This symbol is used in the following list]

$\stackrel{\leftarrow}{F}$

\begin{tabular}{|c|c|c|c|c|c|c|c|c|c|}
\hline \multirow{2}{*}{$\begin{array}{l}\text { Date } \\
(1963)\end{array}$} & \multicolumn{3}{|c|}{ Time } & \multirow{2}{*}{$\begin{array}{l}\text { Magni- } \\
\text { tude }\end{array}$} & \multirow{2}{*}{$\begin{array}{l}\text { Depth } \\
(\mathrm{km})\end{array}$} & \multicolumn{3}{|c|}{ Epicenter } & \multirow{2}{*}{$\begin{array}{c}\text { Felt } \\
\text { Report }\end{array}$} \\
\hline & $\underline{\mathrm{h}}$ & $\underline{\mathrm{m}}$ & $\underline{S}$ & & & Lat. N. & Long. W. & Description & \\
\hline Oct. $\begin{array}{l}1 \\
1 \\
4 \\
4\end{array}$ & $\begin{array}{l}00 \\
11 \\
06 \\
07\end{array}$ & $\begin{array}{l}06 \\
04 \\
39 \\
04\end{array}$ & $\begin{array}{l}04.2 \\
04.3 \\
04.1 \\
33 \cdot 3\end{array}$ & $\begin{array}{l}2.4 \\
2.2 \\
2.0 \\
2.0\end{array}$ & $\begin{array}{l}30 \\
10 \\
----\end{array}$ & $\begin{array}{l}19^{\circ} 08.5^{\prime} \\
19^{\circ} 17.1^{\prime} \\
-10 .\end{array}$ & $\begin{array}{l}155^{\circ} 26.6^{\prime} \\
155^{\circ} 05.4^{\prime} \\
- \\
-\end{array}$ & $\begin{array}{l}7 \mathrm{~km} \mathrm{SE} \text { of Pahala } \\
48 \mathrm{~km} \mathrm{~S} \text {. of Hilo } \\
\mathrm{KM} 30 \ldots \\
\mathrm{KM} 30 \ldots\end{array}$ & 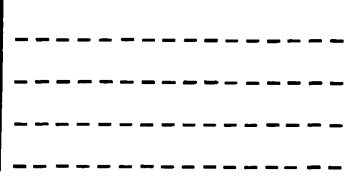 \\
\hline
\end{tabular}

5 Shallow harmonic tremor and earthquakes start at 03:16. Although hundreds of earthquakes

were recorded during the eruption, their wave arrivals were obscured by strong harmonic tremor and few epicenters could be located precisely. These shocks originated mainly along or south/of the upper and middle sections of the east rift of Kilauea.

\begin{tabular}{l|l|l|l|l|l|l|l|l}
5 & 03 & 32 & 23.0 & 3.1 & 3 & $19^{\circ} 22.6^{\prime}$ & $155^{\circ} 08.1^{\prime}$ & $5 \mathrm{~km}$ ENE of Makaopuhi
\end{tabular}

503

\begin{tabular}{l|l|l}
40 & 17.0 & 3.4
\end{tabular}

\begin{tabular}{l|l|l}
3 & $19^{\circ} 22.6^{\prime}$ & $155^{\circ} 09.1^{\prime}$
\end{tabular} seismometer.

$4 \mathrm{~km} \mathrm{NE}$ of Makaopuhi seismometer.

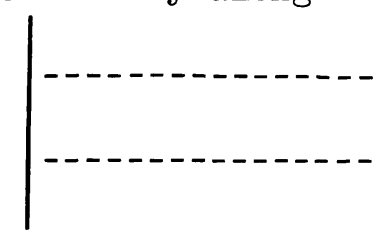


Table 4.--Local earthquakes recorded by seismographs of the U.S. Geological Survey, October, November, and December, 1963--Continued

\begin{tabular}{|c|c|c|c|c|c|c|c|c|c|}
\hline \multirow{2}{*}{$\begin{array}{l}\text { Date } \\
(1963)\end{array}$} & \multicolumn{3}{|c|}{ Time } & \multirow{2}{*}{$\begin{array}{l}\text { Magni - } \\
\text { tude }\end{array}$} & \multirow{2}{*}{$\begin{array}{r}\text { Depth } \\
(\mathrm{km})\end{array}$} & \multicolumn{3}{|c|}{ Epicenter } & \multirow{2}{*}{$\begin{array}{l}\text { Felt } \\
\text { Report }\end{array}$} \\
\hline & $\underline{\mathrm{h}}$ & $\underline{m}$ & $\underline{\mathrm{s}}$ & & & Lat. N. & Long. W. & Description & \\
\hline Oct. 5 & 03 & 56 & 14.5 & 2.9 & 3 & $19^{\circ} 22.0^{\prime}$ & $155^{\circ} 08.0^{\prime}$ & $5 \mathrm{~km} \mathrm{E}$ of Makaopuhi & \\
\hline 5 & 03 & 58 & 08.6 & 3.1 & 3 & $19^{\circ} 22.6^{\prime}$ & $155^{\circ} 08.9^{\prime}$ & $4 \mathrm{~km}$ ENE of Makaopuhi & $-------1----$ \\
\hline 5 & 04 & 14 & 04.2 & $3 \cdot 3$ & 3 & $19^{\circ} 22.2^{\prime}$ & $155^{\circ} 09.0^{\prime}$ & $3 \mathrm{~km}$ ENE of Makaopuhi & $----1---1----$ \\
\hline 5 & 04 & 20 & 41.4 & 2.6 & 3 & $19^{\circ} 22.6^{\prime}$ & $155^{\circ} 08.9^{\prime}$ & $4 \mathrm{~km}$ ENE of Makaopuhi & $--1-1-1-1-1-1$ \\
\hline 5 & 04 & 24 & 59.5 & 3.5 & 3 & $19^{\circ} 22.0^{\prime}$ & $155^{\circ} 08.0^{\prime}$ & $\begin{array}{l}5 \mathrm{~km} \mathrm{E} \text { of Makaopuhi } \\
\text { seismometer. }\end{array}$ & 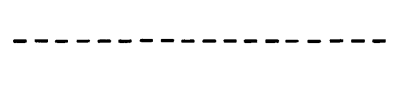 \\
\hline 5 & 04 & 51 & 07.5 & 2.7 & 3 & $19^{\circ} 22.8^{\prime}$ & $155^{\circ} 08.5^{\prime}$ & $4 \mathrm{~km}$ ENE of Makaopuhi & - - - - \\
\hline 5 & 05 & 44 & 41.7 & 2.6 & 3 & $19^{\circ} 22.8^{\prime}$ & $155^{\circ} 06.2^{\prime}$ & $\begin{array}{l}\text { seismometer. } \\
8 \mathrm{~km} \text { ENE of Makaopuhi }\end{array}$ & - \\
\hline 5 & 10 & 25 & 57.9 & 3.0 & 3 & $19^{\circ} 20.7^{\prime}$ & $155^{\circ} 15.0^{\prime}$ & $\begin{array}{l}4 \mathrm{~km} \text { SE. of Ahua } \\
\text { seismometer. }\end{array}$ & - - \\
\hline 5 & 10 & 41 & 22.5 & 3.1 & $<3$ & $19^{\circ} 20.4^{\prime}$ & $155^{\circ} 17.5^{\prime}$ & $\begin{array}{l}5 \mathrm{~km} \mathrm{SW} \text {. of Ahua } \\
\text { seismometer. }\end{array}$ & - - \\
\hline
\end{tabular}


Table 4.--Local earthquakes recorded by seismographs of the U.S. Geological Survey, October, November, and December, 1963--Continued

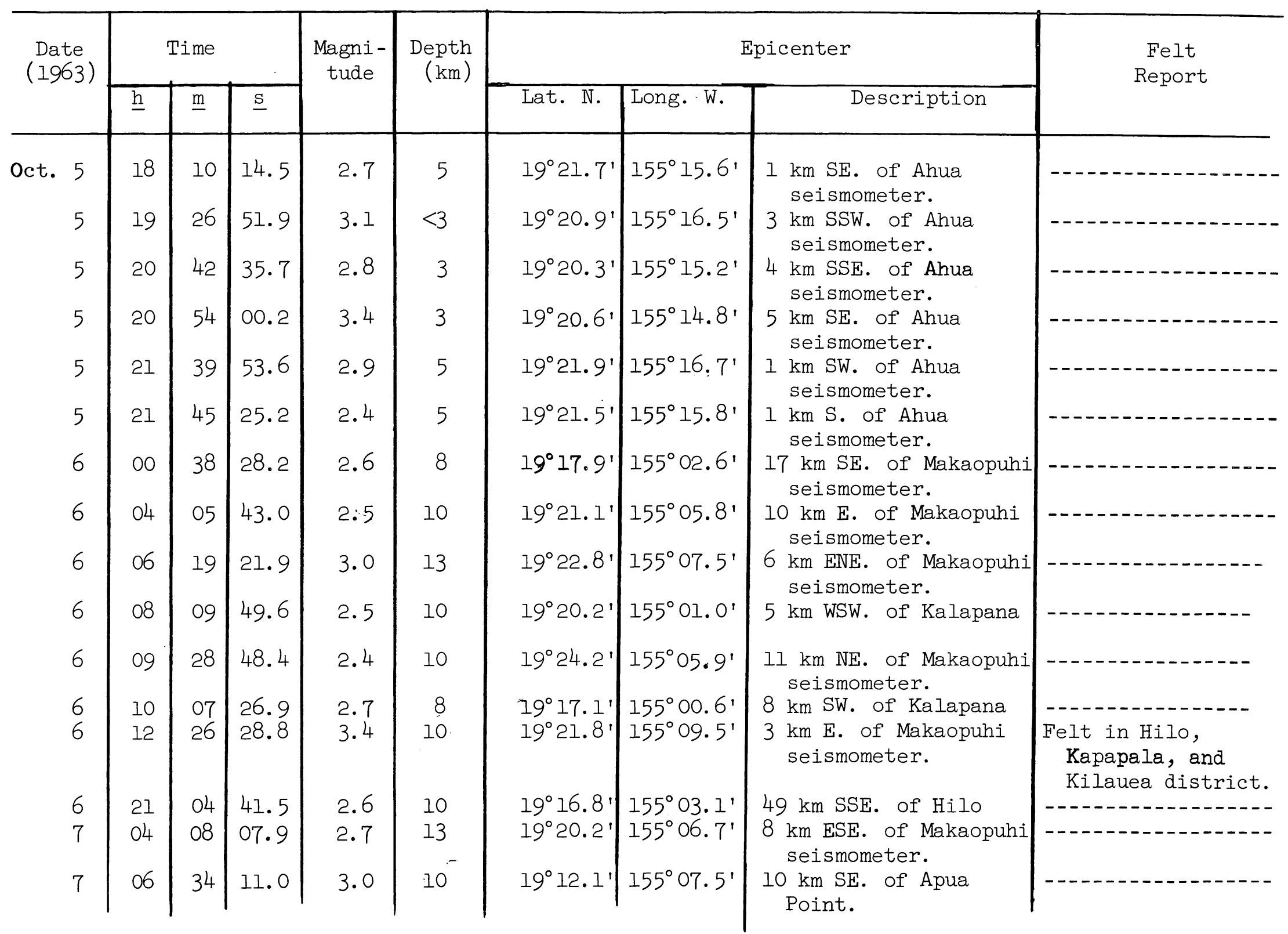


Table 4.--Local earthquakes recorded by seismographs of the U.S. Geological Survey, October, November, and December, 1963--Continued

\begin{tabular}{|c|c|c|c|c|c|c|c|c|c|}
\hline \multirow{2}{*}{$\begin{array}{l}\text { Date } \\
\text { (1963 }\end{array}$} & \multicolumn{3}{|c|}{ Time } & \multirow{2}{*}{$\begin{array}{l}\text { Magni - } \\
\text { tude }\end{array}$} & \multirow{2}{*}{$\begin{array}{r}\text { Depth } \\
(\mathrm{km})\end{array}$} & \multicolumn{3}{|c|}{ Epicenter } & \multirow{2}{*}{$\begin{array}{c}\text { Felt } \\
\text { Report }\end{array}$} \\
\hline & $\underline{\mathrm{h}}$ & $\underline{m}$ & $\underline{\mathrm{s}}$ & & & Lat. N. & Long. W. & Description & \\
\hline ct. 7 & 17 & 05 & 15.3 & 2.5 & 8 & $19^{\circ} 19.8^{\prime}$ & $155^{\circ} 03.7^{\prime}$ & $45 \mathrm{~km}$ SSE. of Hilo & \\
\hline & 20 & $1 \longdiv { 4 }$ & 48.8 & 2.5 & 10 & $19^{\circ} 18.8^{\prime}$ & $155^{\circ} 05.8^{\prime}$ & $45 \mathrm{~km} \mathrm{S.} \mathrm{of} \mathrm{Hilo}$ & $---1-1-1-1$ \\
\hline 7 & 21 & 40 & 28.4 & 2.6 & 5 & $19^{\circ} 12.4^{\prime}$ & $155^{\circ} 10.8^{\prime}$ & $7 \mathrm{~km}$ SSE. of Apua Point & $-----1-00-0=-0$. \\
\hline 7 & 22 & 20 & 33.7 & 3.7 & 5 & $19^{\circ} 12 \cdot 5^{\prime}$ & $155^{\circ} 10.5^{\prime}$ & $7 \mathrm{~km}$ SSE. of Apua Point & $\begin{array}{l}\text { Felt in Kilaua } \\
\text { district. }\end{array}$ \\
\hline 8 & 06 & 10 & 52.0 & 2.0 & -- & ------ & ------- & KM $30 \ldots \ldots$ & 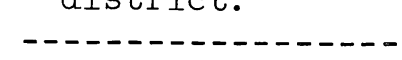 \\
\hline 8 & 11 & 44 & 35.3 & 3.3 & 8 & $19^{\circ} 18.6^{\prime}$ & $155^{\circ} 05.1^{\prime}$ & $45 \mathrm{~km} \mathrm{S.} \mathrm{of} \mathrm{Hilo \ldots \ldots ..........}$ & $\begin{array}{l}\text { Felt in Kilauea } \\
\text { district. }\end{array}$ \\
\hline 9 & 03 & 15 & 04.1 & 2.6 & 10 & $19^{\circ} 17.9^{\prime}$ & $155^{\circ} 13.3^{\prime}$ & $5 \mathrm{~km} \mathrm{NW}$. of Apua Point & 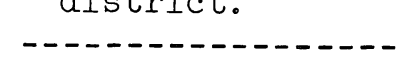 \\
\hline 9 & 18 & 45 & 24.0 & 2.0 & 3 & $19^{\circ} 06.0^{\prime}$ & $155^{\circ} 08.2^{\prime}$ & $18 \mathrm{~km} \mathrm{SE}$. of Apua Point & $---1-1-1--1--1$ \\
\hline 9 & 19 & 05 & 27.0 & 2.2 & 3 & $19^{\circ} 06.0^{\prime}$ & $155^{\circ} 08.2^{\prime}$ & $18 \mathrm{~km} \mathrm{SE}$. of Apua Point & 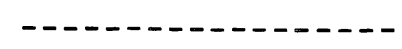 \\
\hline 10 & 00 & 07 & 05.6 & 2.5 & 8 & $19^{\circ} 15.7^{\prime}$ & $155^{\circ} 01.2^{\prime}$ & $5 \mathrm{~km}$ SW. of Kalapana & $-1--1--1-1-1-1-1$ \\
\hline 10 & 21 & 34 & 29.6 & 2.8 & 8 & $19^{\circ} 08.1^{\prime}$ & $155^{\circ} 44.0$ & $18 \mathrm{~km} \mathrm{NW}$. of Naalehu--- & $---1--1--1---1--$ \\
\hline 10 & 20 & 02 & 18.2 & $2 \cdot 3$ & 35 & $19^{\circ} 11.2^{\prime}$ & $155^{\circ} 28.1^{\prime}$ & $2 \mathrm{~km} \mathrm{SE}$. of Pahala--.-- & Felt in Pahala...- \\
\hline 11 & 11 & 04 & 35.7 & 2.0 & 8 & $19^{\circ} 05.8^{\prime}$ & $155^{\circ} 23.7^{\prime}$ & $21 \mathrm{~km}$ ENE. of Naalehu-- & 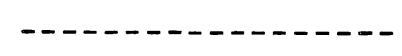 \\
\hline 11 & 13 & 40 & 11.2 & 2.4 & 3 & $19^{\circ} 15.4^{\prime}$ & $155^{\circ} 20.1^{\prime}$ & $\begin{array}{l}\text { ll km SE. of Desert } \\
\text { seismometer. }\end{array}$ & $-\ldots-\ldots-\ldots$ \\
\hline 11 & 13 & 40 & 48.9 & 2.6 & 3 & $19^{\circ} 15.4^{\prime}$ & $155^{\circ} 20.1^{\prime}$ & $\begin{array}{l}11 \mathrm{~km} \text { SE. of Desert } \\
\text { seismometer. }\end{array}$ & Felt in Kapapala \\
\hline 11 & 16 & 52 & 47.8 & 3.8 & 8 & $19^{\circ} 08.3^{\prime}$ & $155^{\circ} 05.0^{\prime}$ & $64 \mathrm{~km} \mathrm{~S}$. of Hilo & $\begin{array}{l}\text { Felt in Kilauea } \\
\text { district. }\end{array}$ \\
\hline 11 & 17 & 37 & 34.4 & 2.2 & 8 & $19^{\circ} 08.3^{\prime}$ & $155^{\circ} 05.0^{\prime}$ & $64 \mathrm{~km} \mathrm{~S}$. of Hilo & $---1--1--1---1$ \\
\hline 11 & 17 & 38 & 16.3 & 2.3 & 10 & $19^{\circ} 14^{\circ} 8^{\prime}$ & $155^{\circ} 07.7^{\prime}$ & $7 \mathrm{~km}$ ESE. of Apua Point. & $---1----1----$ \\
\hline 11 & 22 & 44 & 22.4 & 2.2 & 8 & $19^{\circ} 12.0$ & $155^{\circ} 21.1^{\prime}$ & $\begin{array}{l}16 \mathrm{~km} \text { SSE. of Desert } \\
\text { seismometer. }\end{array}$ & ------------ \\
\hline 11 & 23 & 46 & 01.3 & 3.2 & 13 & $21^{\circ} 10^{\prime}$ & $155^{\circ} 20^{\prime}$ & $\begin{array}{l}106 \mathrm{~km} \mathrm{NE} \text {. of Haleakala } \\
\text { seismometer. }\end{array}$ & $---1----\cdots$ \\
\hline 12 & 16 & 39 & 39.2 & 2.8 & 8 & $19^{\circ} 06.6^{\prime}$ & $155^{\circ} 06.4^{\prime}$ & $67 \mathrm{~km} \mathrm{~S}$. of Hilo.......... & 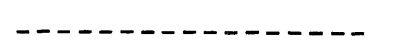 \\
\hline 12 & 18 & 14 & 25.6 & 2.8 & 8 & $19^{\circ} 14.5^{\prime}$ & $155^{\circ} 22.0$ & $\begin{array}{l}11 \mathrm{~km} \text { S. of Desert } \\
\text { seismometer. }\end{array}$ & $-\ldots-\ldots-n-\cdots$ \\
\hline 12 & 21 & 16 & 30.3 & 3.3 & 8 & $19^{\circ} 14.5^{\prime}$ & $155^{\circ} 22.3^{\prime}$ & $\begin{array}{l}11 \mathrm{~km} \text { S. of Desert } \\
\text { seismometer. }\end{array}$ & ------------ \\
\hline
\end{tabular}


Table 4.--Local earthquakes recorded by seismographs of the U.S. Geological Survey, October, November, and December, 1963--Continued

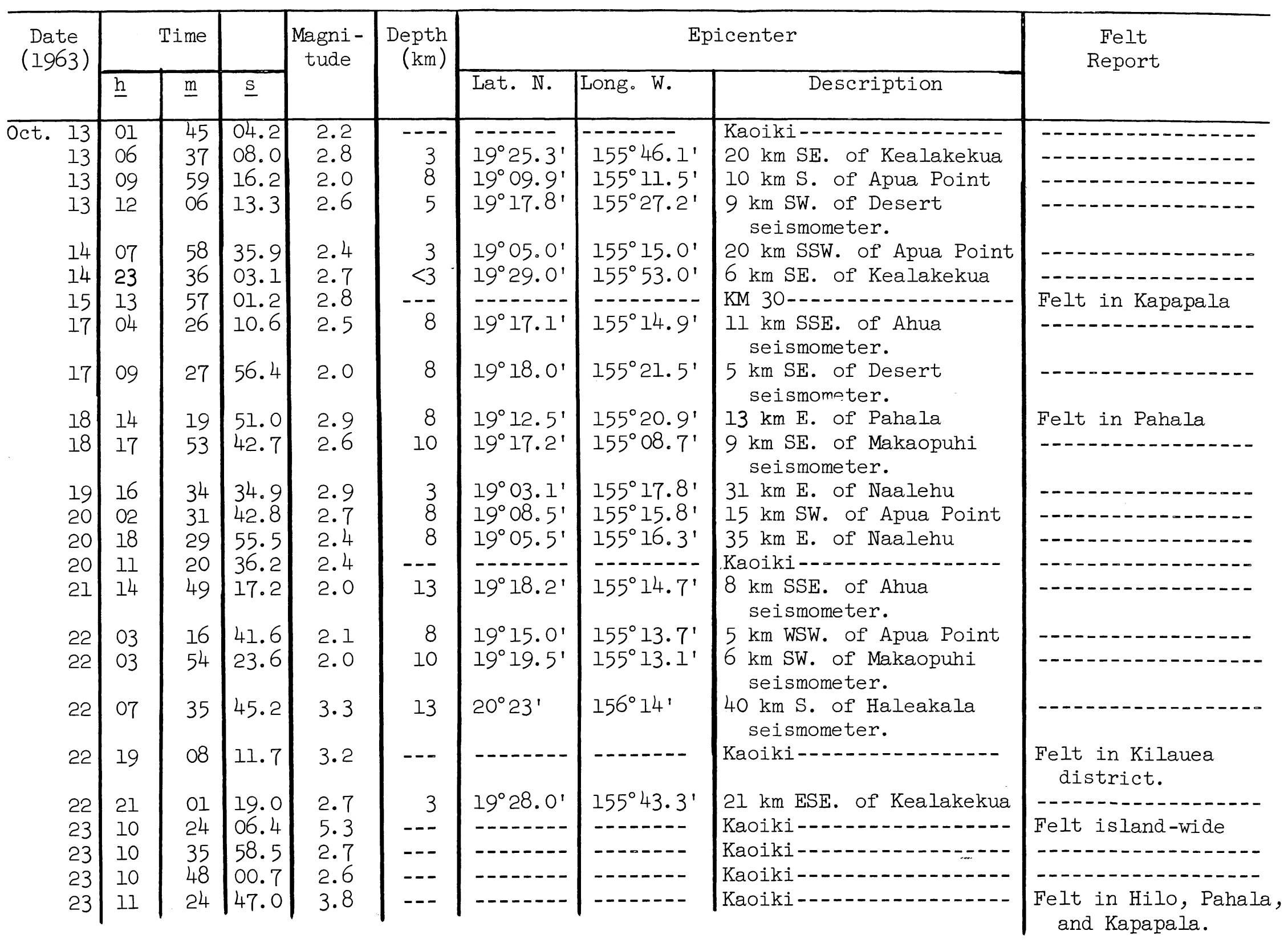


Table 4. - -Local earthquakes recorded by seismographs of the U.S. Geological Survey, October, November, and December, 1963--Continued

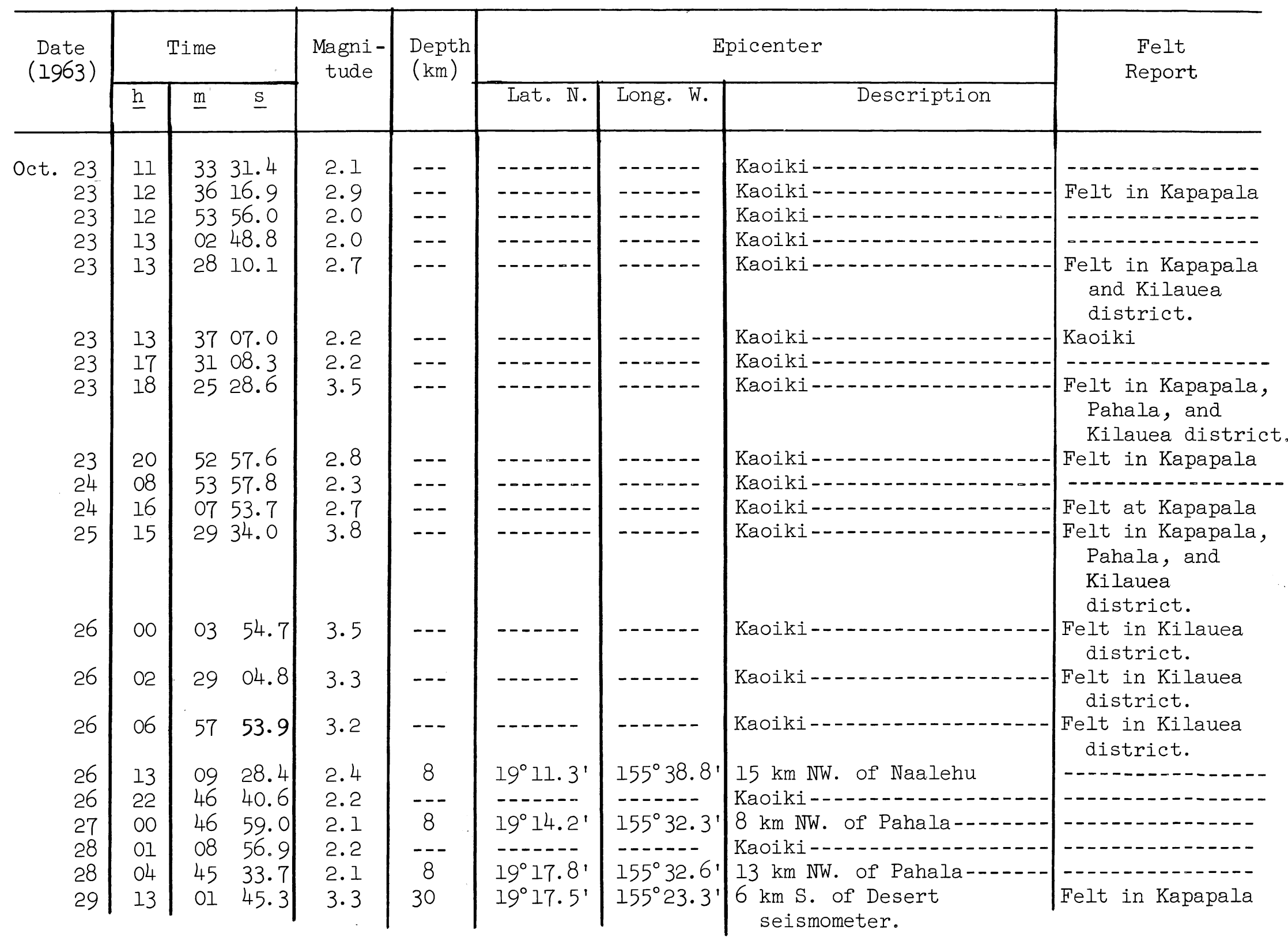


Table 4. - - Local earthquakes recorded by seismographs of the U.S. Geological Survey, October, November, and December, 1963--Continued

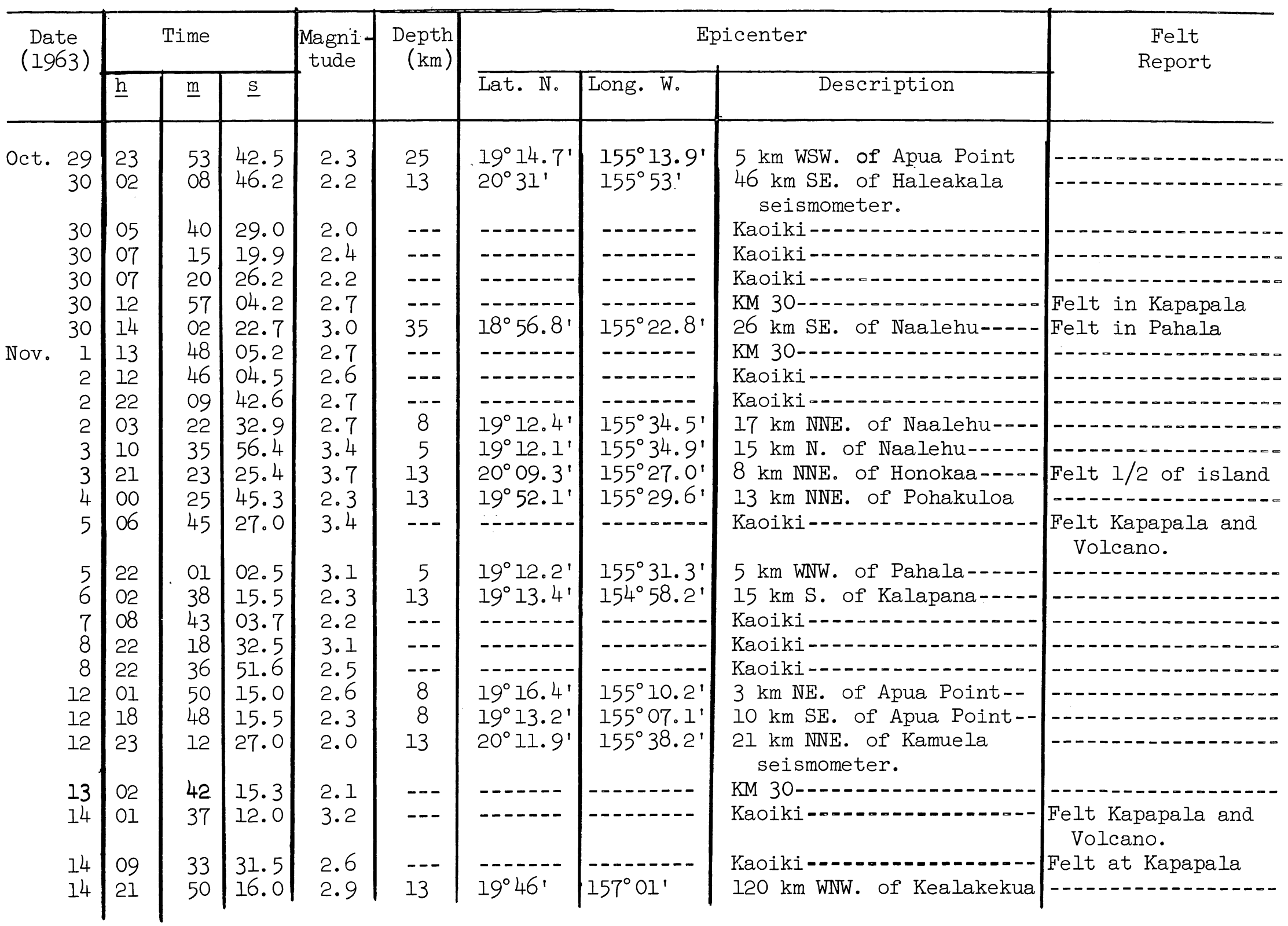


Table 4.--Local earthquakes recorded by seismographs of the U.S. Geological Survey, October, November, and December, 1963--Continued

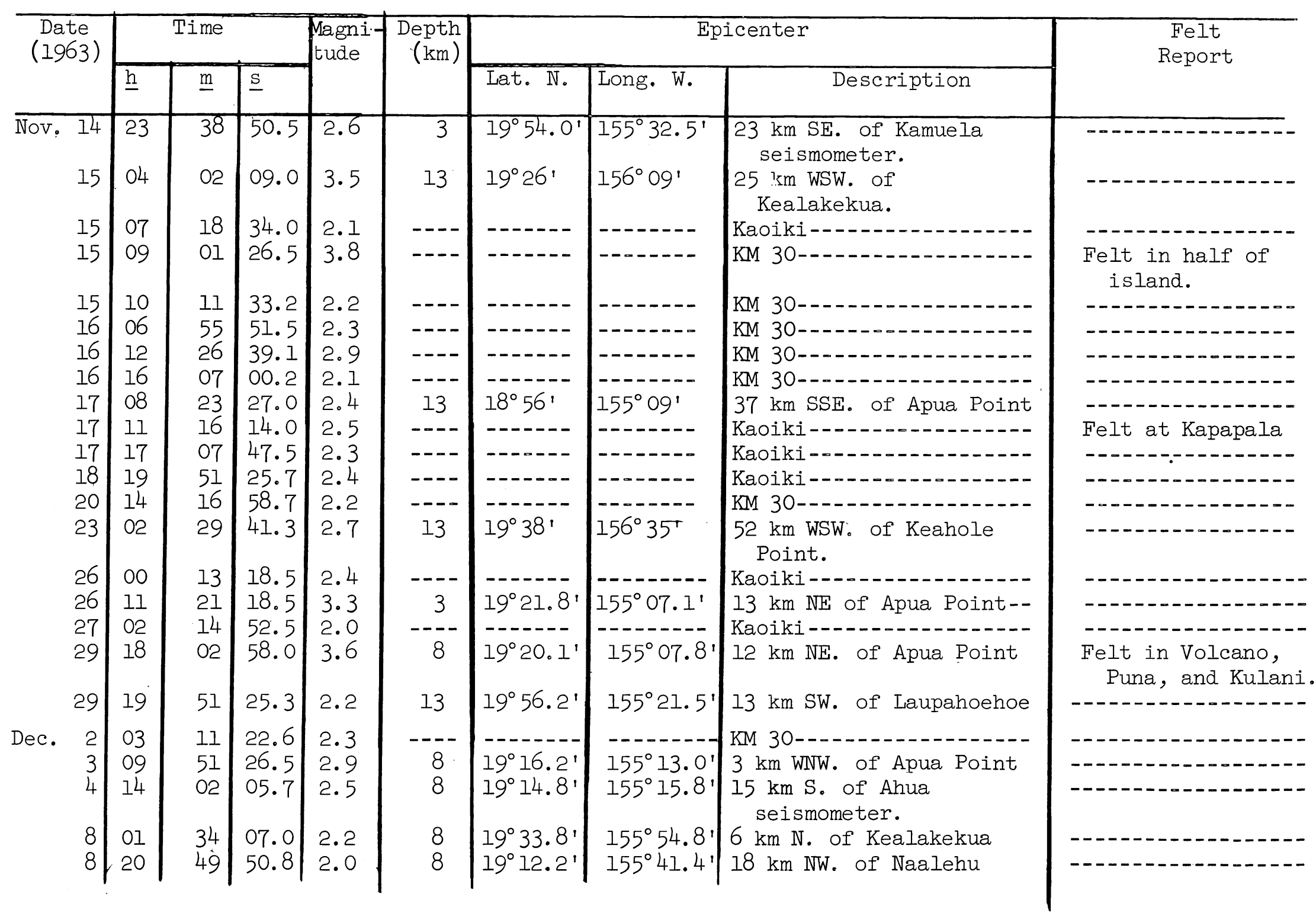


Table 4.--Local earthquakes recorded by seismographs of the U.S. Geological Survey, October, November, and December, 1963 --Continued

\begin{tabular}{|c|c|c|c|c|c|c|c|c|c|}
\hline \multirow{2}{*}{$\begin{array}{l}\text { Date } \\
(1963)\end{array}$} & \multicolumn{3}{|c|}{ Time } & \multirow{2}{*}{$\begin{array}{l}\text { Magni } \\
\text { tude }\end{array}$} & \multirow{2}{*}{$\begin{array}{r}\text { Depth } \\
(\mathrm{km})\end{array}$} & \multicolumn{3}{|r|}{ Epicenter } & \multirow{2}{*}{$\begin{array}{c}\text { Felt } \\
\text { Report }\end{array}$} \\
\hline & $\underline{\mathrm{h}}$ & $\underline{\mathrm{m}}$ & $\underline{s}$ & & & Lat. N. & Long. W. & Description & \\
\hline Dec. 9 & 18 & 47 & 55.5 & 3.4 & 8 & $19^{\circ} 53.1^{\prime}$ & $155^{\circ} 31.3^{\prime}$ & $26 \mathrm{~km} \mathrm{SE}$. of Kamuela-.- & Felt in half of \\
\hline 9 & 18 & 50 & 07.0 & 2.5 & 8 & $19^{\circ} 56.7^{\prime}$ & $155^{\circ} 30.6^{\prime}$ & $23 \mathrm{~km}$ ESE. of Kamuela-- & $---------1----\infty$ \\
\hline 11 & 22 & 22 & 24.0 & 2.6 & ---- & ------- & $-\cdots-1-1$ & Kaoiki-n- & 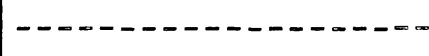 \\
\hline 12 & 17 & 29 & 19.0 & 2.3 & 8 & $19^{\circ} 18.8^{\prime}$ & $155^{\circ} 10.4^{\prime}$ & $8 \mathrm{~km}$ NNE. of Apua Point & 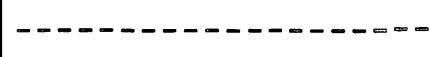 \\
\hline 13 & 21 & 19 & 24.0 & 2.4 & 8 & $19^{\circ} 17.8^{\prime}$ & $155^{\circ} 17.5^{\prime}$ & $\begin{array}{l}12 \mathrm{~km} \text { ESE. of Desert } \\
\text { seismometer. }\end{array}$ & $-\infty---------n---\infty, \infty$ \\
\hline 14 & 03 & 32 & 34.0 & 2.2 & --- & $----\infty$ & ------- & 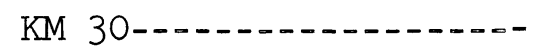 & $------------n$ \\
\hline 14 & 22 & 16 & 04.7 & 2.2 & 8 & $19^{\circ} 14.1{ }^{\prime}$ & $155^{\circ} 36.7^{\prime}$ & $19 \mathrm{~km} \mathrm{NNW}$ of Naalehu-.- & 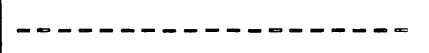 \\
\hline 14 & 23 & 59 & 22.1 & 2.3 & 13 & $19^{\circ} 59.2^{\prime}$ & $155^{\circ} 59.2^{\prime}$ & $18 \mathrm{~km}$ WSW. of Kawaihae-- & 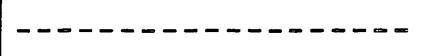 \\
\hline 16 & 10 & 57 & 39.8 & 2.6 & 8 & $19^{\circ} 13.1$ & $155^{\circ} 34.8^{\prime}$ & $17 \mathrm{~km} \mathrm{~N}$. of Naalehu----- & 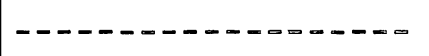 \\
\hline 19 & 21 & 17 & 30.0 & $2 \cdot 3$ & 13 & $19^{\circ} 57.0^{\prime}$ & $155^{\circ} 22.2^{\prime}$ & $15 \mathrm{~km}$ WSW. of Laupahoehoe & 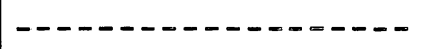 \\
\hline 21 & 00 & 30 & 58.4 & 2.1 & --- & $-\infty-\infty-\infty$ & $-\infty-\infty-\infty$ & KM 30 & $---1--1--n-\infty$ \\
\hline 21 & 06 & 06 & 11.1 & 2.8 & $-\infty$ & $-\ldots-\infty-n$ & $--\ldots-n-1$ & $\mathrm{KM} 3 \mathrm{O}-\ldots+\ldots$ & $-\infty---\infty-\infty-\infty-\infty-\infty-\infty---$ \\
\hline 23 & 16 & 49 & 19.4 & 2.2 & --- & $---\cdots$ & $---1---$ & Kaoiki_-_on & $--\infty---0---\infty-=-\infty---\infty$ \\
\hline 23 & 17 & 44 & 55.5 & 2.6 & 8 & $19^{\circ} 19.2^{\prime}$ & $155^{\circ} 12.7^{\prime}$ & $\begin{array}{l}10 \mathrm{~km} \mathrm{SE} \text { of Ahua } \\
\text { seismometer. }\end{array}$ & 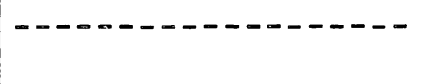 \\
\hline 24 & 04 & 33 & 37.0 & 2.0 & 3 & $19^{\circ} 18.1^{\prime}$ & $155^{\circ} 05.8^{\prime}$ & $12 \mathrm{~km}$ ENE. of Apua Point & 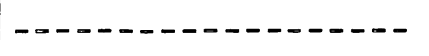 \\
\hline 24 & 20 & 04 & 55.5 & $2 \cdot 3$ & 8 & $19^{\circ} 14.9^{\prime}$ & $155^{\circ} 11.3^{\prime}$ & $2 \mathrm{~km}$ SSE. of Apua Point & $-----------\infty=----1$ \\
\hline 25 & 04 & 20 & 04.0 & 2.3 & 8 & $19^{\circ} 14.5^{\prime}$ & $155^{\circ} 25.9^{\prime}$ & $\begin{array}{l}12 \mathrm{~km} \text { SSW. of Desert } \\
\text { seismometer. }\end{array}$ & $------------\cdots---$ \\
\hline 26 & 12 & 11 & 21.0 & 2.7 & ---- & ------- & ------ & Kaoiki-1-n & 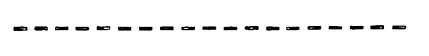 \\
\hline 27 & 15 & 39 & 31.1 & 2.7 & 13 & $20^{\circ} 01.2^{\prime}$ & $155^{\circ} 53.0^{\prime}$ & $7 \mathrm{~km}$ WSW. of Kawaihae--- & 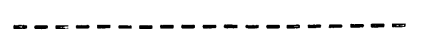 \\
\hline 27 & 02 & 12 & 30.6 & 2.0 & --- & ------ & $-\ldots---$ & KM 30-n. & 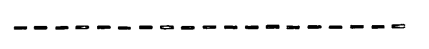 \\
\hline 27 & 03 & 03 & 23.3 & 2.0 & --- & ----- & $---\ldots--$ & KM 30-n. & 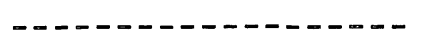 \\
\hline 27 & 17 & 33 & 15.0 & 2.4 & --- & ------ & ------ & KM 30- & 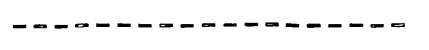 \\
\hline 28 & 11 & 14 & 55.5 & 2.4 & --- & & & Kaoiki-n-n-n & - - - - - - - - - - \\
\hline 29 & 04 & 02 & 02.1 & 2.4 & 25 & $19^{\circ} 19.8^{\prime}$ & $155^{\circ} 12.8^{\prime}$ & $\begin{array}{l}8 \mathrm{~km} \mathrm{SE} \text {. of Ahua } \\
\text { seismometer. }\end{array}$ & 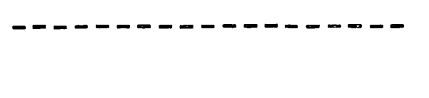 \\
\hline
\end{tabular}


Table 4. --Local earthquakes recorded by seismographs of the U.S. Geological Survey, October, November, and December, 1963--Continued

\begin{tabular}{|c|c|c|c|c|c|c|c|c|c|}
\hline \multirow{2}{*}{$\begin{array}{c}\text { Date } \\
(1963)\end{array}$} & \multicolumn{3}{|c|}{ Time } & \multirow{2}{*}{$\begin{array}{l}\text { Magni- } \\
\text { tude }\end{array}$} & \multirow{2}{*}{$\begin{array}{l}\text { Depth } \\
(\mathrm{km})\end{array}$} & \multicolumn{3}{|c|}{ Epicenter } & \multirow{2}{*}{$\begin{array}{l}\text { Felt } \\
\text { Report }\end{array}$} \\
\hline & $\underline{\mathrm{h}}$ & $\underline{\mathrm{m}}$ & $\underline{\mathrm{s}}$ & & & Lat. N. & Long. W. & Description & \\
\hline $\begin{aligned} & \text { Dec. } 29 \\
& 29 \\
& 31\end{aligned}$ & $\begin{array}{l}16 \\
22 \\
03 \\
09\end{array}$ & $\begin{array}{l}22 \\
14 \\
18 \\
24\end{array}$ & $\begin{array}{l}19.0 \\
50.3 \\
07.0 \\
56.3\end{array}$ & $\begin{array}{l}2.4 \\
2.7 \\
2.3 \\
3.1\end{array}$ & $\begin{array}{r}---- \\
----\end{array}$ & $\begin{array}{l}-19^{\circ} 12.7^{\prime} \\
-\cdots-\cdots\end{array}$ & $155^{\circ} 11.0$ & $\begin{array}{l}\text { Kaoiki } \\
\text { Kaoiki } \\
6 \text { km SSE. of Apua } \\
\text { Point. }\end{array}$ & 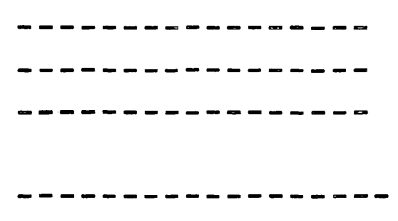 \\
\hline
\end{tabular}


Table 5.--Distant earthquakes

[Times are reported in Greenwich Civil Time which is 10 hours faster than Hawaiian Standard Time. A "c" following the time of $P$ indicates compressional first motion; a "d" indicates dilatational first motion. Station symbols, locations, and instrumentation are presented in summary. 29. Magnitudes calculated from the Hawaii seismograms are followed by (HVO). Location of epicenter, origin times, and focal depths, and magnitudes reported by other institutions are taken from "Preliminary Determination of Epicenters" published by the U.S. Coast and Geodetic Survey]

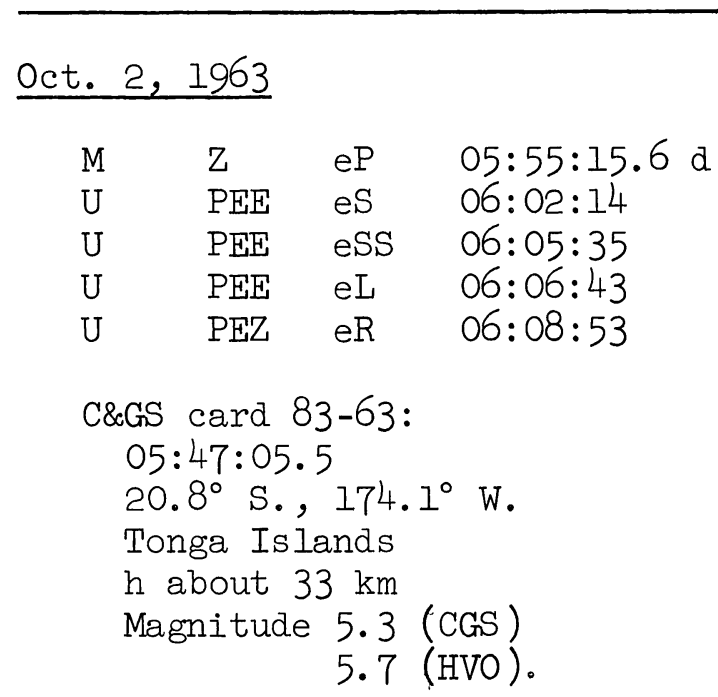

Oct. 3

U PEZ eR 16:48:07

C\&GS card 80-63:

$15: 48: 17.2$

$58.5^{\circ}$ S., $25.1^{\circ} \mathrm{W}$.

Sandwich Is lands

$\mathrm{h}$ about $54 \mathrm{~km}$.

Oct. 3

$\begin{array}{lllcc}M & Z & \text { eP } & 23: 35: 19.9 & c \\ U & \text { PEZ } & \text { iP } & 21 & c \\ U & \text { PEZ } & \text { eR } & 23: 54: 49 & \\ U & \text { PEE } & \text { iS } & 23: 44: 09 & \end{array}$

C\&GS card 80-63:

$23: 24: 34.7$

$32.2^{\circ} \mathrm{N}_{0}, 131.6^{\circ} \mathrm{E}$.

Kyushu, Japan

$\mathrm{h}$ about $33 \mathrm{~km}$

Magnitude 6.5 (Pas)

5.7 (CGS).
Oct. 7

$\begin{array}{lllr}\text { M } & \text { Z } & \text { iP } & 13: 22: 24.9 \mathrm{~d} \\ \mathrm{~A} & \mathrm{Z} & \text { iP } & 24.4 \mathrm{~d} \\ \mathrm{D} & \mathrm{Z} & \text { iP } & 23.1 \mathrm{~d} \\ \mathrm{~N} & \mathrm{Z} & \text { iP } & 24.4 \mathrm{~d} \\ \mathrm{MP} & \mathrm{Z} & \text { iP } & 24.7 \mathrm{~d} \\ \mathrm{Na} & \mathrm{Z} & \text { iP } & 20.9 \mathrm{~d} \\ \mathrm{Ka} & \mathrm{Z} & \text { iP } & 27.6 \mathrm{c} \\ \mathrm{Hi} & \mathrm{Z} & \text { iP } & 27.3 \mathrm{~d} \\ \mathrm{Ha} & \text { Z } & \text { iP } & 29.1 \mathrm{c}\end{array}$

C\&GS card 81-63:

$13: 14: 24.6$

$23.6^{\circ} \mathrm{S} ., 179.9^{\circ} \mathrm{E}$.

Fiji Islands region.

$h$ about $550 \mathrm{~km}$

Magnitude 5.7 (CGS).

Oct. 8

$\begin{array}{lllr}M & Z & \text { iP } & 00: 24: 25.3 \mathrm{c} \\ \mathrm{A} & \mathrm{Z} & \text { iP } & 23.9 \mathrm{c} \\ \mathrm{D} & \mathrm{Z} & \text { iP } & 23.2 \mathrm{c} \\ \mathrm{N} & \mathrm{Z} & \text { iP } & 24.8 \mathrm{c} \\ \mathrm{MP} & \mathrm{Z} & \text { iP } & 25.2 \mathrm{c} \\ \mathrm{Na} & \mathrm{Z} & \text { iP } & 21.0 \mathrm{c} \\ \mathrm{Hi} & \mathrm{Z} & \text { iP } & 27.6 \mathrm{c} \\ \mathrm{Ha} & \text { Z } & \text { iP } & 31.1 \mathrm{c}\end{array}$

C\&GS card 81-63:

$00: 17: 01.1$

$15.1^{\circ}$ S., $173.2^{\circ} \mathrm{W}$.

Samoa Islands region

$\mathrm{h}$ about $33 \mathrm{~km}$

Magnitude 6 (Pas)

5.75 (Bks)

5.7 (CGS). 


\begin{tabular}{|c|c|c|c|}
\hline \multicolumn{4}{|c|}{ Oct. 12, 1963} \\
\hline M & Z & iP & $11: 36: 09.4 \mathrm{c}$ \\
\hline D & $\mathrm{Z}$ & iP & $10.1 \mathrm{c}$ \\
\hline $\mathrm{N}$ & Z & $\mathrm{eP}$ & $10.3 \mathrm{c}$ \\
\hline $\mathrm{Pa}$ & Z & $\mathrm{eP}$ & $10.6 \mathrm{~d}$ \\
\hline $\mathrm{Na}$ & Z & $\mathrm{eP}$ & $14.7 \mathrm{~d}$ \\
\hline $\mathrm{Ka}$ & Z & $\mathrm{eP}$ & $05.2 \mathrm{c}$ \\
\hline $\mathrm{Hi}$ & Z & $\mathrm{eP}$ & $10.0 \mathrm{c}$ \\
\hline $\mathrm{Ha}$ & $\mathrm{Z}$ & $\mathrm{eP}$ & $11: 35: 57.5 \mathrm{c}$ \\
\hline $\mathrm{U}$ & PEZ & iP & $11: 36: 10$ d \\
\hline $\mathrm{U}$ & PEZ & iS & $11: 43: 31$ \\
\hline $\mathrm{U}$ & PEZ & $i R$ & $11: 50: 30$ \\
\hline
\end{tabular}

C\&GS card 85-63:

$11: 26: 57.9$

$44.8^{\circ} \mathrm{N} ., 149.0^{\circ} \mathrm{E}$.

Kurile Islands

$h$ about $40 \mathrm{~km}$

Magnitude 6.75-7 (Pas)

7 (Bks)
$6.75-7$ (Pal)
7.5 (HVO).

Oct. 13

$\begin{array}{lllrl}M & Z & \text { iP } & 05: 27: 03.6 & \mathrm{~d} \\ \mathrm{~A} & \mathrm{Z} & \mathrm{eP} & 04.7 \mathrm{~d} \\ \mathrm{D} & \mathrm{Z} & \mathrm{eP} & 04.2 \mathrm{~d} \\ \mathrm{U} & \mathrm{Z} & \mathrm{eP} & 03.9 \mathrm{~d} \\ \mathrm{Na} & \mathrm{Z} & \mathrm{iP} & 06.0 \mathrm{c} \\ \mathrm{Hi} & \mathrm{Z} & \mathrm{eP} & 01.3 \mathrm{~d} \\ \mathrm{Ha} & \mathrm{Z} & \mathrm{eP} & 05: 26: 52.7 & \mathrm{~d} \\ \mathrm{U} & \mathrm{PEZ} & \text { iP } & 05: 27: 04 & \mathrm{~d} \\ \mathrm{U} & \text { PEZ } & \text { iS } & 05: 34: 26 & \\ \mathrm{Ha} & \mathrm{Z} & \text { Tmax } & 06: 21: 20 & \end{array}$

C\&GS card 84-63:

$05: 17: 57.1$

$44.8^{\circ}$ N., $149.5^{\circ} \mathrm{E}$.

Kurile Islands

$\mathrm{h}$ about $60 \mathrm{~km}$

Felt: Hokkaido and Honshu,

Japan. Seismic sea waves:

4.0 ft. Hokkaido; 2.5 ft.

Oahu, Hawaii; 2.2 ft.

Crescent City, California;

$1.5 \mathrm{ft}$. Attu, Aleutians.

Magnitude 8.25 (Pas)

7.75-8 (Pal)

8.1 (HVO)

Small tsunami in Hilo Bay.

oct. 13

$\begin{array}{lllr}M & Z & \text { iP } & 13: 07: 24.9 \mathrm{~d} \\ \text { A } & \text { Z } & \text { iP } & 26.1 \mathrm{~d} \\ \text { D } & \text { Z } & \text { iP } & 25.1 \mathrm{~d} \\ \text { E } & \text { Z } & \text { iP } & 26.2 \mathrm{~d} \\ \text { U } & \text { Z } & \text { iP } & 25.2 \mathrm{~d} \\ \text { Hi } & \text { Z } & \text { iP } & 24.8 \mathrm{~d}\end{array}$

C\&GS card 84-63:

$12: 58: 21.6$

45. $0^{\circ} \mathrm{N}_{0}, 150.1^{\circ} \mathrm{E}$.

Kurile Islands

$\mathrm{h}$ about $50 \mathrm{~km}$

Magnitude 5.4 (CGS).

Oct. 13

$\begin{array}{lllr}\text { M } & \text { Z } & \text { iP } & 16: 08: 56.7 \mathrm{c} \\ \text { WP } & \text { Z } & \text { iP } & 57.2 \mathrm{c} \\ \text { MP } & \text { Z } & \text { iP } & 57.9 \mathrm{c} \\ \text { Hi } & \text { Z } & \text { eP } & 56.3 \mathrm{c} \\ \text { U } & \text { PEN } & \text { iS } & 16: 16: 24 \\ \text { U } & \text { PEE } & \text { iL } & 16: 21: 14 \\ \text { U } & \text { PEZ } & \text { iR } & 16: 23: 22\end{array}$

C\&GS card -84-63:

$15: 59: 52.9$

$45.6^{\circ}$ N., $150.5^{\circ} \mathrm{E}$.

Kurile Islands

$\mathrm{h}$ about $35 \mathrm{~km}$

Magnitude 6.1 (CGS).

oct. $13(14)$

U PEZ eR 00:17:50

C\&GS card 84-63:

$23: 52: 22.8$

44. $5^{\circ}$ N., $150.1^{\circ} \mathrm{E}$.

Kurile Islands

$\mathrm{h}$ about $50 \mathrm{~km}$

Magnitude 5.5 (CGS).

Oct. 14

$\begin{array}{llll}D & Z & \text { eP } & 04: 20: 15.5 \text { d } \\ \text { U } & \text { PEZ } & \text { eR } & 04: 35: 06\end{array}$ 
Table 5.--Distant earthquakes--Continued

Oct. 14, 1963--Continued

C\&GS card 84-63:

$04: 11: 14.0$

$44.7^{\circ}$ N., $150.6^{\circ} \mathrm{E}$.

Kurile Islands

$\mathrm{h}$ about $45 \mathrm{~km}$

Magnitude 5.3 (CGS).

Oct. 14

\begin{tabular}{|c|c|c|c|}
\hline M & Z & iP & $13: 30: 41.7 \mathrm{~d}$ \\
\hline A & Z & $\mathrm{eP}$ & $43.0 \mathrm{~d}$ \\
\hline D & Z & iP & 42.7 \\
\hline $\mathrm{MP}$ & Z & $e P$ & 43.7 \\
\hline $\mathrm{U}$ & $\mathrm{Z}$ & iP & 42.8 \\
\hline $\mathrm{Pa}$ & Z & iP & 44.1 \\
\hline $\mathrm{Na}$ & Z & iP & 43.0 \\
\hline $\mathrm{Hi}$ & Z & iP & 42.2 \\
\hline $\mathrm{Ha}$ & Z & iP & 1.3 \\
\hline $\mathrm{U}$ & PEN & $i G$ & $13: 42: 51$ \\
\hline $\mathrm{U}$ & PEZ & eR & $13: 44: 51$ \\
\hline
\end{tabular}

C\&GS card 84-63:

$13: 21: 45.2$

$44.8^{\circ} \mathrm{N} ., 151.0^{\circ} \mathrm{E}$.

Kurile Islands

$\mathrm{h}$ about $60 \mathrm{~km}$

Magnitude 5.75 (Bks)

5.9 (CGS)
6.0 (HVO).

Oct. 15

U PEZ eR 07:13:23

C\&GS card 90-63:

$06: 49: 37.8$

42. $2^{\circ}$ N., $152.3^{\circ} \mathrm{E}$.

Kurile Islands

$\mathrm{h}$ about $40 \mathrm{~km}$

Magnitude 4.2 (CGS)

5.8 (HVO).

Oct. 17

$\begin{array}{llrr}\text { M } & \text { Z } & \text { iP } & 23: 33: 57.8 \mathrm{~d} \\ \mathrm{~A} & \mathrm{Z} & \text { iP } & 59.4 \mathrm{~d} \\ \mathrm{D} & \mathrm{Z} & \text { iP } & 58.7 \mathrm{~d} \\ \mathrm{~Pa} & \mathrm{Z} & \text { eP } & 23: 34: 00.8 \mathrm{~d} \\ \mathrm{U} & \text { PEN } & \text { iS } & 23: 41: 17\end{array}$

Oct. 17--Continued

$\begin{array}{llll}\text { U } & \text { PEN } & \text { IG } & 23: 46: 09 \\ \text { U } & \text { PEZ } & \text { iR } & 23: 48: 23\end{array}$

C\&GS card 84-63:

$23: 24: 34.4$

$44.6^{\circ}$ N., $149.0^{\circ}$ E.

Kurile Islands

$\mathrm{h}$ about $45 \mathrm{~km}$

Magnitude 5.4 (CGS)

Oct. 20

\begin{tabular}{|c|c|c|c|}
\hline M & Z & iP & 01:02:20.6 \\
\hline A & Z & $\mathrm{eP}$ & 22.0 \\
\hline D & Z & iP & 21.3 \\
\hline $\mathrm{Ha}$ & Z & $\mathrm{eP}$ & 14.4 \\
\hline $\mathrm{Na}$ & Z & $e P$ & 22.8 \\
\hline $\mathrm{Hi}$ & Z & $\mathrm{eP}$ & 20.3 \\
\hline $\mathrm{U}$ & PEZ & iP & 22 \\
\hline $\mathrm{U}$ & PEZ & is & $01: 09: 36$ \\
\hline $\mathrm{U}$ & PEZ & $i R$ & $01: 16: 10$ \\
\hline $\mathrm{U}$ & PEN & $i G$ & $01: 14: 00$ \\
\hline
\end{tabular}

C\&GS card 85-63:

$00: 53: 07.2$

$44.7^{\circ}$ N., $150.7^{\circ} \mathrm{E}$.

Kurile Islands

$\mathrm{h}$ about $25 \mathrm{~km}$

Seismic sea waves: $1.0 \mathrm{ft}$.

LaJolla, California; 0.5 ft. Oahu,

Hawaii.

Magnitude 6.75-7 (Pas)

$7.25-7.5$ (PaI)

Oct. 20

$\begin{array}{lllr}M & Z & \text { iP } & 09: 19: 48.7 \mathrm{c} \\ \mathrm{A} & \mathrm{Z} & \text { 1P } & 49.7 \mathrm{c} \\ \mathrm{D} & \mathrm{Z} & \text { iP } & 49.3 \mathrm{c} \\ \mathrm{MP} & \mathrm{Z} & \text { iP } & 50.1 \mathrm{c} \\ \mathrm{U} & \mathrm{Z} & \text { iP } & 49.3 \mathrm{c} \\ \mathrm{Pa} & \mathrm{Z} & \text { iP } & 50.5 \mathrm{c} \\ \mathrm{Na} & \mathrm{Z} & \text { iP } & 49.4 \mathrm{c} \\ \mathrm{Hi} & \text { Z } & \text { iP } & 48.9 \mathrm{c}\end{array}$

C\&GS card 87-63:

$09: 10: 43.9$

$44.4^{\circ}$ N., $150.0^{\circ} \mathrm{E}$. 
Table 5.--Distant earthquakes--Continued

Oct. 20, 1963--Continued

C\&GS card--Continued:

Kurile Islands

h about $40 \mathrm{~km}$

Magnitude 5.5 (CGS).

Oct. 20

U PEZ eR 12:16:01

C\&GS card 87-63:

$11: 52: 20.7$

$44.7^{\circ}$ N., $150.2^{\circ}$ E.

Kurile Islands

$\mathrm{h}$ about $45 \mathrm{~km}$

Magnitude 4.2 (CGS)

Oct. 22

$\begin{array}{llll}\mathrm{Pa} & \mathrm{Z} & \mathrm{Tmax} & 05: 16: 20 \\ \mathrm{Ha} & \mathrm{Z} & \mathrm{Tmax} & 05: 14: 14\end{array}$

C\&GS card 85-63:

04:29:11. 7

$51.0^{\circ}$ N., $179.4^{\circ} \mathrm{E}$.

$\mathrm{h}$ about $55 \mathrm{~km}$

Rat Islands, Aleutian Islands

Magnitude 4.9 (CGS)

Oct. 22

U PEZ eR 03:49:26

C\&GS card 87-63:

$03: 25: 38.8$

43. $9^{\circ}$ N., $150.3^{\circ} \mathrm{E}$.

Kurile Islands

$\mathrm{h}$ about $50 \mathrm{~km}$

Magnitude 4.9 (CGS).

Oct. 25

$\begin{array}{lllr}\text { M } & \text { Z } & \text { iP } & 20: 09: 05.2 \mathrm{c} \\ \text { A } & \text { Z } & \text { iP } & 05.4 \mathrm{c} \\ \text { D } & \text { Z } & \text { iP } & 04.8 \mathrm{c} \\ \text { MP } & \text { Z } & \text { eP } & 06.9 \mathrm{c} \\ \text { U } & \text { Z } & \text { iP } & 05.2 \mathrm{c} \\ \mathrm{Ha} & \text { Z } & \text { eP } & 20: 08: 59.1 \mathrm{c}\end{array}$

oct. 25--Continued

C\&GS card 89-63:

19:58:58.3

$12.3^{\circ}$ N., $144.5^{\circ}$ E.

Mariana Is lands

$\mathrm{h}$ about $29 \mathrm{~km}$

Magnitude 5.4 (CGS).

Oct. 29

Ha Z Tmax 07:43:07

C\&GS card 88-63:

$07: 01: 42.7$

$40.4^{\circ}$ N. , $124.7^{\circ} \mathrm{W}$.

Near coast of Humbolt County, California

$\mathrm{h}$ about $38 \mathrm{~km}$

Magnitude 4.7 (CGS)

Oct. 31

M Z eP 03:26:00.6 c

A Z eP 03:25:58.0 C

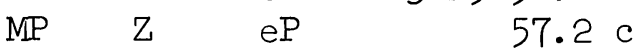

$\begin{array}{llll}\mathrm{U} & \mathrm{U} & \mathrm{P} & \mathrm{C}\end{array}$

$\begin{array}{llll}\mathrm{Pa} & \mathrm{Z} & \mathrm{eP} & 59.7 \mathrm{c}\end{array}$

$\mathrm{Na} \quad \mathrm{Z} \quad \mathrm{eP} \quad 56.7 \mathrm{~d}$

$\mathrm{Ka} \quad \mathrm{Z}$ eP 03:26:03.1 c

$\mathrm{Ke} Z \mathrm{Z} \quad \mathrm{eP} \quad 00.1 \mathrm{C}$

$\mathrm{Hi}$ Z eP $02.1 \mathrm{c}$

$U$ PEE ES 03:32:4I

U PEE eG 03:36:00

C\&GS card 89-63:

$03: 17: 42.0$

$21.8^{\circ}$ S., $175.0^{\circ} \mathrm{W}$.

Tonga Is lands

$\mathrm{h}$ about $33 \mathrm{~km}$

Magnitude 6.25 (Pas)

5.5 (Bks)

$6.25-6.5 \cdot(\mathrm{Pal})$

5.2 (CGS)

5.9 (HVO). 


\begin{tabular}{|c|c|c|c|}
\hline \multicolumn{4}{|c|}{ Oct. 31,1963} \\
\hline M & Z & iP & $08: 58: 53.2 \mathrm{~d}$ \\
\hline D & Z & $e P$ & $52.0 \mathrm{~d}$ \\
\hline $\mathrm{U}$ & $\mathrm{Z}$ & iP & $52.1 \mathrm{~d}$ \\
\hline $\mathrm{Pa}$ & $\mathrm{Z}$ & iP & $54.1 \mathrm{~d}$ \\
\hline $\mathrm{Ka}$ & $\mathrm{Z}$ & iP & $56.7 \mathrm{c}$ \\
\hline $\mathrm{Ke}$ & $\mathrm{Z}$ & iP & $52.7 \mathrm{c}$ \\
\hline $\mathrm{Hi}$ & $\mathrm{Z}$ & iP & $55.3 \mathrm{c}$ \\
\hline $\mathrm{Ha}$ & $\mathrm{Z}$ & iP & $57.9 \mathrm{c}$ \\
\hline
\end{tabular}

C\&GS card 89-63:

$08: 51: 42$

$17.9^{\circ}$ S., $178.8^{\circ} \mathrm{W}$.

Fiji Islands region

h about $637 \mathrm{~km}$

Magnitude 4.3 (CGS).

Nov. 3

$\begin{array}{lllr}\text { M } & Z & \text { iP } & 03: 22: 20.9 \mathrm{~d} \\ \mathrm{~A} & \mathrm{Z} & \text { iP } & 19.8 \mathrm{~d} \\ \mathrm{D} & \mathrm{Z} & \text { iP } & 20.0 \mathrm{~d} \\ \mathrm{MP} & \mathrm{Z} & \text { iP } & 19.5 \mathrm{~d} \\ \mathrm{U} & \mathrm{Z} & \text { iP } & 20.2 \mathrm{~d} \\ \mathrm{~Pa} & Z & \text { iP } & 18.8 \mathrm{~d} \\ \mathrm{Na} & Z & \text { iP } & 20.1 \mathrm{~d} \\ \mathrm{Ka} & Z & \text { iP } & 22.4 \mathrm{~d} \\ \mathrm{Ke} & \text { Z } & \text { iP } & 21.7 \mathrm{~d} \\ \mathrm{Hi} & Z & \text { iP } & 20.5 \mathrm{~d} \\ \mathrm{Ha} & \text { Z } & \text { iP } & 25.0 \mathrm{~d} \\ \mathrm{U} & \text { PEN } & \text { iS } & 03: 32: 32 \\ \mathrm{U} & \text { PEN } & \text { iG } & 03: 42: 56 \\ \mathrm{U} & \text { PEZ } & \text { iR } & 03: 46: 10\end{array}$

C\&GS card 90-63:

$03: 10: 12.7$

$3.5^{\circ}$ S., $77.8^{\circ} \mathrm{W}$.

Peru-Ecuador border

$\mathrm{h}$ about $33 \mathrm{~km}$

Magnitude 6.75 (Pas)

$6-6.25$ (Bks)

6.0 (Pal)

6. 0 (CGS).

Nov. 4

6.3 (HVO).

Nov. 4--Continued

$\mathrm{Ha}$ Z iP $\quad 19.8 \mathrm{c}$

C\&GS card 91-63:

$01: 14: 32.8$

$15.1^{\circ} \mathrm{S} ., 167.3^{\circ} \mathrm{E}$.

New Hebrides Is lands

$\mathrm{h}$ about $154 \mathrm{~km}$

Magnitude 6.75-7 (Pas)

5.8 (CGS).

Nov. 4

\begin{tabular}{|c|c|c|}
\hline Z & $\mathrm{eP}$ & 01:29:03.6 \\
\hline Z & $\mathrm{eP}$ & 04.9 \\
\hline Z & iP & 03.6 \\
\hline $\mathrm{MP}$ & $e P$ & 04.5 \\
\hline Z & iP & 04.8 \\
\hline $\mathrm{Pa}$ & iP & 06.4 \\
\hline $\mathrm{Na}$ & iP & 02.0 \\
\hline $\mathrm{Ke}$ & iP & 01.6 \\
\hline $\mathrm{Hi}$ & $e P$ & 06.7 \\
\hline PEZ & iP & $01: 29: 05$ \\
\hline PEZ & iPcP & $01: 29: 17$ \\
\hline PEZ & ipP & $01: 29: 34$ \\
\hline PEZ & isP & $01: 29: 50$ \\
\hline PEZ & i & $01: 32: 42$ \\
\hline PEZ & $i$ & $01: 35: 00$ \\
\hline $\mathrm{PEE}$ & is & $01: 38: 53$ \\
\hline $\mathrm{PEE}$ & iSS & $01: 44: 05$ \\
\hline PEN & iSSS & $01: 47: 42$ \\
\hline PEN & $i G$ & $01: 49: 51$ \\
\hline
\end{tabular}

C\&GS card 91-63:

$01: 17: 08.9$

$6.8^{\circ} \mathrm{S} ., 129.6^{\circ} \mathrm{E}$.

Banda Sea

$\mathrm{h}$ about $80 \mathrm{~km}$

Felt throughout Australia, northern Tasmania and Papua Magnitude 7.5 (HVO). 
Table 5.--Distant earthquakes--Continued

\begin{tabular}{clll}
\cline { 1 - 1 } Nov. 6, 1963 & & \\
\cline { 1 - 1 } & & & \\
M & Z & iP & $02: 24: 25.1 \mathrm{c}$ \\
U & Z & eP & $24.5 \mathrm{c}$ \\
U & PEE & iS & $02: 33: 19$ \\
U & PEN & eL & $02: 40: 35$ \\
U & PEZ & iR & $02: 44: 43$
\end{tabular}

C\&GS card 93-63:

$02: 13: 16.8$

$138.4^{\circ} \mathrm{E} ., 2.6^{\circ} \mathrm{S}$.

Western New Guinea

$\mathrm{h}$ about $33 \mathrm{~km}$

Magnitude 6.3 (HVO).

Nov. 8

$\begin{array}{lllr}M & Z & \text { iP } & 16: 25: 05.6 \mathrm{~d} \\ M P & Z & \text { iP } & 06.0 \mathrm{~d}\end{array}$

C\&GS card 91-63:

$16: 13: 36.2$

$128.4^{\circ}$ E. , $2.7^{\circ} \mathrm{N}$.

Molucca Passage

$\mathrm{h}$ about $216 \mathrm{~km}$

Magnitude 5.7 (CGS).

Nov. 9

\begin{tabular}{|c|c|c|c|}
\hline M & Z & $\mathrm{eP}$ & $21: 27: 17.4$ \\
\hline A & Z & eP & 16.5 \\
\hline $\mathrm{MP}$ & Z & eP & 15.9 \\
\hline $\mathrm{Pa}$ & Z & $\mathrm{eP}$ & 15.0 \\
\hline $\mathrm{Ka}$ & $\mathrm{Z}$ & $\mathrm{eP}$ & 18.1 \\
\hline $\mathrm{Hi}$ & Z & $\mathrm{eP}$ & 15.9 \\
\hline $\mathrm{Ha}$ & $\mathrm{Z}$ & iP & 21.8 \\
\hline U & PEZ & iP & 17 \\
\hline U & $\mathrm{PEZ}$ & ipP & $21: 29: 25$ \\
\hline U & PEZ & esP & $21: 30: 31$ \\
\hline U & PEZ & iPP & $21: 31: 09$ \\
\hline U & PEZ & ipPP & $21: 32: 55$ \\
\hline $\mathrm{U}$ & PEZ & iPPP & $21: 33: 43$ \\
\hline $\mathrm{U}$ & $\mathrm{PEZ}$ & $i$ & $21: 39: 35$ \\
\hline U & $\mathrm{PEE}$ & iSKS & $21: 36: 51$ \\
\hline $\mathrm{U}$ & $\mathrm{PEE}$ & iSP & $21: 38: 19$ \\
\hline $\mathrm{U}$ & $\mathrm{PEE}$ & is $P$ & $21: 41: 01$ \\
\hline $\mathrm{U}$ & PEN & iS & $21: 37: 15$ \\
\hline $\mathrm{U}$ & PEN & $i$ & $21: 42: 19$ \\
\hline $\mathrm{U}$ & PEN & iSS & $21: 43: 31$ \\
\hline $\mathrm{U}$ & PEN & iSSS & $21: 46: 47$ \\
\hline U & PEN & $i$ & $21: 49: 55$ \\
\hline $\mathrm{U}$ & PEN & eG & $21: 52: 13$ \\
\hline
\end{tabular}

Nov. 9--Continued

C\&GS card 93-63:

$21: 15: 30.4$

$9.0^{\circ}$ S., $71.5^{\circ} \mathrm{W}$.

Western Brazil

$\mathrm{h}$ about $600 \mathrm{~km}$

Magnitude 6.75-7 (Pas)

5.9 (CGS)

7 (HV).

Nov. 10

$\begin{array}{lllr}M & Z & \text { eP } & 01: 12: 26.8 \mathrm{c} \\ \mathrm{A} & \mathrm{Z} & \mathrm{eP} & 26.3 \mathrm{c} \\ \mathrm{MP} & \mathrm{Z} & \mathrm{eP} & 25.8 \mathrm{c} \\ \mathrm{Pa} & \mathrm{Z} & \text { iP } & 25.0 \mathrm{c} \\ \mathrm{Hi} & \mathrm{Z} & \text { iP } & 25.8 \mathrm{c} \\ \mathrm{Ha} & \mathrm{Z} & \mathrm{eP} & 32.0 \mathrm{c} \\ \mathrm{U} & \mathrm{PEE} & \text { iS } & 01: 22: 19 \\ \mathrm{U} & \mathrm{PEE} & \text { iSP } & 01: 23: 34 \\ \mathrm{U} & \mathrm{PEE} & \text { i } & 01: 26: 58\end{array}$

C\&GS card 93-63:

$01: 00: 38.8$

$9.2^{\circ}$ S., $71.5^{\circ} \mathrm{W}$.

Western Brazil

$\mathrm{h}$ about $600 \mathrm{~km}$

Magnitude 6.5-6.75 (Pas)

$$
\begin{aligned}
& 6.25 \text { (BKS) } \\
& 5.6 \text { (CGS). }
\end{aligned}
$$

Nov. 10

$\begin{array}{llll}\text { M } & \text { Z } & \text { eP } & 17: 27: 05.0 \mathrm{c} \\ \text { D } & \text { Z } & \text { eP } & 05.5 \mathrm{c} \\ \text { U } & \text { Z } & \text { eP } & 05.2 \mathrm{c} \\ \text { U } & \text { PEN } & \text { iS } & 17: 34: 45 \\ \text { U } & \text { PEN } & \text { eI } & 17: 39: 19 \\ \text { U } & \text { PEZ } & \text { iR } & 17: 41: 43\end{array}$

C\&GS card 93-63:

$17: 17: 42.7$

$44.4^{\circ} \mathrm{N} ., 149.0^{\circ} \mathrm{E}$.

Kurile Islands

$h$ about $40 \mathrm{~km}$

Magnitude 5.5 (CGS)

6.4 (HVO).

Nov. 15

$\begin{array}{lllr}M & Z & \text { eP } & 21: 15: 40.6 \mathrm{c} \\ \mathrm{A} & \mathrm{Z} & \mathrm{eP} & 42.7 \mathrm{c} \\ \mathrm{D} & \mathrm{Z} & \mathrm{iP} & 42.6 \mathrm{c}\end{array}$


Table 5.--Distant earthquakes--Continued

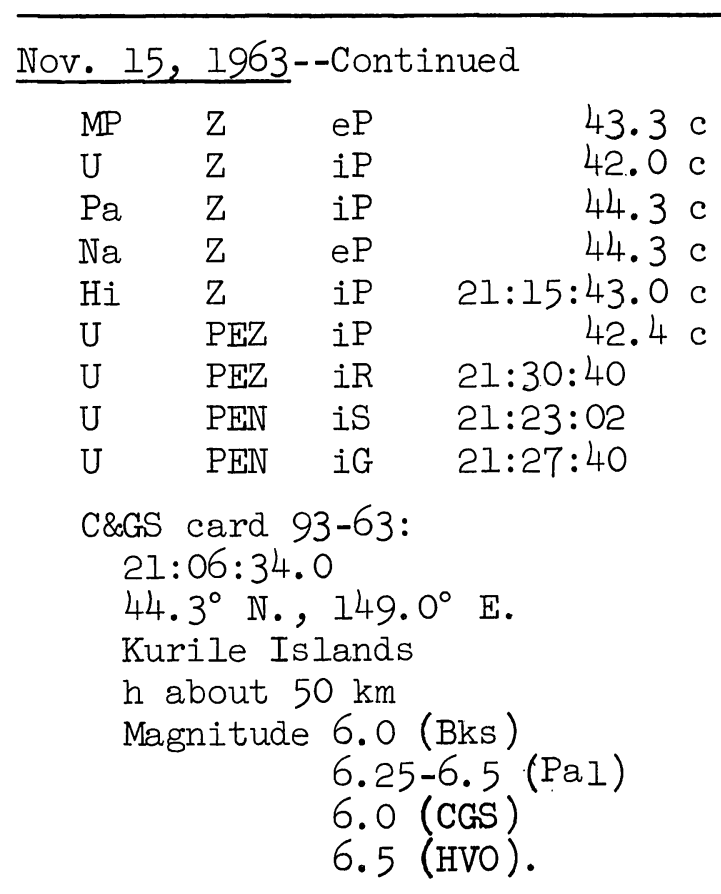

Nov. 18

\begin{tabular}{|c|c|c|c|}
\hline M & Z & iP & $14: 45: 58.7 \mathrm{~d}$ \\
\hline A & Z & eP & $58.3 \mathrm{~d}$ \\
\hline D & Z & eP & $59.3 \mathrm{~d}$ \\
\hline$M P$ & Z & iP & $57.9 \mathrm{~d}$ \\
\hline $\mathrm{U}$ & Z & iP & $58.1 \mathrm{~d}$ \\
\hline $\mathrm{Pa}$ & Z & iP & $56.8 \mathrm{c}$ \\
\hline $\mathrm{Na}$ & Z & iP & $14: 46: 03.1 \mathrm{c}$ \\
\hline $\mathrm{Hi}$ & Z & $\mathrm{eP}$ & $14: 45: 56 \quad d$ \\
\hline $\mathrm{Ke}$ & Z & iP & $14: 46: 02.7 \mathrm{c}$ \\
\hline $\mathrm{Ha}$ & Z & $\mathrm{eP}$ & $14: 46: 02.7 \mathrm{c}$ \\
\hline U & PEZ & iS & $14: 51: 56$ \\
\hline U & PEZ & iR & $14: 56: 06$ \\
\hline $\mathrm{U}$ & Z & Tmax & $15: 25: 50$ \\
\hline $\mathrm{Pa}$ & Z & $T \max$ & 41 \\
\hline $\mathrm{Ha}$ & Z & $T \max$ & $15: 26: 15$ \\
\hline
\end{tabular}

C\&GS card 93-63:

$14: 38: 28.9$

$29.9^{\circ}$ N., $113.6^{\circ} \mathrm{W}$.

Gulf of California

$\mathrm{h}$ about $14 \mathrm{~km}$

Magnitude 6.5 (Pas)

$6.5-6.75(\mathrm{PaI})$
5.7 (CGS).
6.5 (HVO).
Nov. 23

$\begin{array}{lllc}\text { M } & Z & \text { iP } & 07: 58: 08.6 \mathrm{c} \\ \text { U } & \text { Z } & \text { eP } & 08.2 \mathrm{c} \\ \text { U } & \text { PEZ } & \text { eR } & 08: 08: 04 \\ \text { M } & Z & \text { Tmax } & 08: 37: 41 \\ U & Z & T \max & 49\end{array}$

C\&GS card 96-63:

$07: 50: 46.3$

30. $1^{\circ}$ N., $114.0^{\circ} \mathrm{W}$.

Gulf of California

$\mathrm{h}$ about $14 \mathrm{~km}$

Magnitude 6 (Pas)

6 (Bks)

5.1 (CGS)

6.0 (HVO).

Nov. 23

$\begin{array}{lllr}\text { M } & \text { Z } & \text { Tmax } & 09: 19: 16 \\ \mathrm{U} & \mathrm{Z} & \mathrm{Tmax} & 12 \\ \mathrm{Ha} & \mathrm{Z} & T \max & 15\end{array}$

C\&GS card 102-63:

$08: 32.31$

$29.9^{\circ} \mathrm{N} ., 114.0^{\circ} \mathrm{W}$.

Gulf of California

$h$ about $14 \mathrm{~km}$

Magnitude 5.3 (CGS).

Nov. 28

Ha Z Tmax 16:02:06

C\&GS card 96-63:

$15: 13: 11.0$

$52.2^{\circ}$ N., $174.2^{\circ}$ E.

Near Islands,

Aleutian Islands

$\mathrm{h}$ about $33 \mathrm{~km}$

Magnitude 5.4 (CGS).

Nov. 30

$\begin{array}{lllr}\text { M } & \text { Z } & \text { iP } & 09: 59: 46.9 \mathrm{~d} \\ \text { A } & \text { Z } & \text { eP } & 47.0 \mathrm{~d} \\ \text { D } & \text { Z } & \text { eP } & 46.2 \mathrm{~d} \\ \text { U } & \text { Z } & \text { iP } & 47.0 \mathrm{~d}\end{array}$

C\&GS card 96-63:

$09: 47: 59.0$

$1.6^{\circ}$ N., $128.4^{\circ} \mathrm{E}$. 
Table 5.--Distant earthquakes--Continued

\section{Nov. 30, 1963--Continued \\ C\&GS card--Continued \\ Halmahera region \\ $h$ about $61 \mathrm{~km}$ \\ Magnitude 5.5 (CGS).}

Dec. 2

$\begin{array}{llrr}\text { M } & \text { Z } & T \max & 07: 40: 15 \\ \text { A } & Z & T \text { max } & 20 \\ \text { MP } & \text { Z } & T \max & 22 \\ \text { U } & \text { Z } & T \text { max } & 21 \\ \text { Pa } & \text { Z } & T \max & 17 \\ \mathrm{Hi} & \mathrm{Z} & T \max & 02 \\ \mathrm{Ha} & \mathrm{Z} & T \max & 07: 39: 24\end{array}$

C\&GS card 97-63:

$06: 52: 20$

$54.1^{\circ}$ N., $134.2^{\circ} \mathrm{W}$.

Queen Charlotte Islands

$\mathrm{h}$ about $33 \mathrm{~km}$

Magnitude 3.9 (CGS).

Dec. $3(4)$

$\begin{array}{llrr}\text { M } & \text { Z } & T \max & 01: 00: 14 \\ \text { A } & \text { Z } & T \max & 02 \\ \text { D } & \text { Z } & T \max & 11 \\ \text { U } & \text { Z } & T \max & 07 \\ \mathrm{~Pa} & \text { Z } & T \max & 00: 59: 43 \\ \mathrm{Ha} & \text { Z } & T \max & 01: 02: 48\end{array}$

C\&GS card 97-63:

$23: 03: 41.6$

$22.4^{\circ} \mathrm{S} ., 69.3^{\circ} \mathrm{W}$.

Northern Chile

$\mathrm{h}$ about $18 \mathrm{~km}$

Magnitude 6.25 (Pas)

6.1 (CGS).

Dec. 4

Ha Z Tmax 02:43:11

C\&GS card 103-63:

$01: 35: 24.5$

$45.3^{\circ}$ N., $153.4^{\circ} \mathrm{E}$.

Kurile Islands

$\mathrm{h}$ about $60 \mathrm{~km}$

Magnitude 4.1 (CGS).
Dec. 7

$\begin{array}{lllr}M & Z & \text { iP } & 04: 15: 40.9 \mathrm{c} \\ \mathrm{A} & \mathrm{Z} & \text { iP } & 40.1 \mathrm{~d} \\ \mathrm{U} & \mathrm{Z} & \text { iP } & 40.4 \mathrm{c} \\ \mathrm{Pa} & \mathrm{Z} & \text { iP } & 41.9 \mathrm{~d} \\ \mathrm{Hi} & \mathrm{Z} & \text { eP } & 42.8 \mathrm{~d} \\ \mathrm{Ke} & \mathrm{Z} & \text { iP } & 38.6 \mathrm{~d}\end{array}$

C\&GS card 99-63:

$04: 07: 52.8$

22. $1^{\circ}$ S., $179.4^{\circ} \mathrm{W}$.

Fiji Islands region

$\mathrm{h}$ about $546 \mathrm{~km}$

Magnitude 5.5 (CGS).

Dec. 7

$\begin{array}{lllr}M & Z & \text { iP } & 04: 55: 36.0 \mathrm{~d} \\ \mathrm{~A} & \mathrm{Z} & \text { iP } & 36.2 \mathrm{c} \\ \mathrm{U} & \mathrm{Z} & \mathrm{eP} & 36.0 \mathrm{c} \\ \mathrm{Pa} & \mathrm{Z} & \text { iP } & 38.0 \mathrm{~d} \\ \mathrm{Hi} & \mathrm{Z} & \mathrm{eP} & 38.4 \mathrm{~d}\end{array}$

C\&GS card 102-63:

$04: 46: 52.2$

$18.8^{\circ} \mathrm{S} ., 169.2^{\circ} \mathrm{E}$.

New Hebrides Islands

$\mathrm{h}$ about $227 \mathrm{~km}$

Magnitude 4.7 (CGS).

Dec. 9

$\begin{array}{lllr}\text { M } & \text { Z } & T \max & 06: 22: 49 \\ \text { A } & \text { Z } & T \max & 47 \\ \text { D } & \text { Z } & T \max & 43 \\ \text { MP } & \text { Z } & T \max & 47 \\ \text { U } & \text { Z } & T \max & 43 \\ \mathrm{~Pa} & \mathrm{Z} & T \max & 48 \\ \mathrm{Hi} & \mathrm{Z} & T \max & 19\end{array}$

C\&GS card 99-63:

$05: 38: 29.5$

$54.9^{\circ}$ N., $159.4^{\circ} \mathrm{W}$.

Alaska Peninsula

$h$ about $45 \mathrm{~km}$

Magnitude 5.1 (CGS). 
Table 5.--Distant earthquakes--Continued

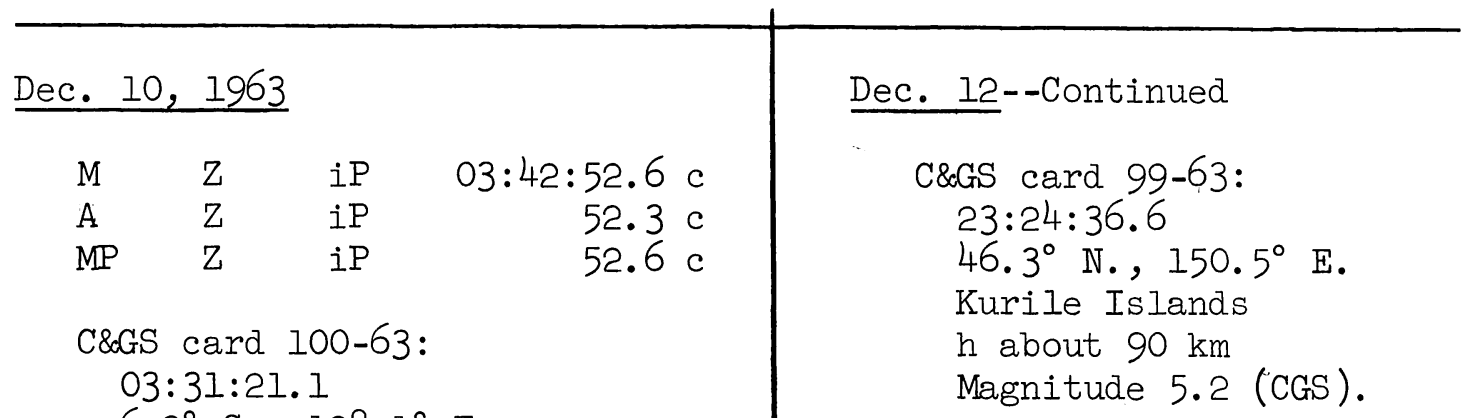

6. $2^{\circ}$ S., 128. $1^{\circ} \mathrm{E}$.

Banda Sea

Felt: Darwin

$\mathrm{h}$ about $366 \mathrm{~km}$

Magnitude 5.6 (CGS).

Dec. 11

$\begin{array}{lllr}\text { A } & \text { Z } & \text { eP } & 00: 55: 10.3 \mathrm{~d} \\ \mathrm{Na} & \mathrm{Z} & \text { iP } & 09.7 \mathrm{~d} \\ \mathrm{Hi} & \mathrm{Z} & \text { eP } & 15.9 \mathrm{c} \\ \mathrm{Ke} & \mathrm{Z} & \text { eP } & 11.2 \mathrm{c} \\ \mathrm{U} & \text { PEZ } & \text { eR } & 01: 05: 17\end{array}$

C\&GS card 101-63:

$00: 47: 48.3$

$15.1^{\circ}$ S., $173.6^{\circ} \mathrm{W}$.

Tonga Islands region

Magnitude 5.6 (CGS):

$\mathrm{h}$ about $33 \mathrm{~km}$.

Dec. 11

$\begin{array}{lllr}M & Z & \text { iP } & 02: 38: 37.4 \mathrm{c} \\ \mathrm{A} & \mathrm{Z} & \text { eP } & 36.4 \mathrm{c} \\ \mathrm{D} & \mathrm{Z} & \text { iP } & 35.8 \mathrm{c} \\ \mathrm{MP} & \mathrm{Z} & \text { iP } & 36.4 \mathrm{c} \\ \mathrm{U} & \mathrm{Z} & \text { iP } & 36.8 \mathrm{c} \\ \mathrm{Na} & \mathrm{Z} & \text { iP } & 33.7 \mathrm{c} \\ \mathrm{Ka} & \text { Z } & \text { iP } & 39.9 \mathrm{c} \\ \mathrm{Ke} & \text { Z } & \text { iP } & 35.5 \mathrm{c} \\ \mathrm{Hi} & \text { Z } & \text { iP } & 39.3 \mathrm{c} \\ \mathrm{Ha} & \text { Z } & \text { iP } & 41.7 \mathrm{c}\end{array}$

C\&GS card 103-63:

$02: 31: 19.4$

$17.8^{\circ} \mathrm{S} ., 178.6^{\circ} \mathrm{W}$.

Fiji Islands

$\mathrm{h}$ about $537 \mathrm{~km}$

Magnitude 4.9 (CGS).

Dec. 12

$\begin{array}{lllrl}M & Z & \text { iP } & 23: 33: 35.0 & \mathrm{~d} \\ D & Z & \text { iP } & 35.4 & \mathrm{~d}\end{array}$

Dec. 14

$\begin{array}{lllr}\text { M } & \text { Z } & \text { iP } & 07: 59: 19.8 \mathrm{c} \\ \text { A } & Z & \text { iP } & 20.4 \mathrm{c} \\ \text { D } & \text { Z } & \text { iP } & 20.7 \mathrm{c} \\ \text { MP } & \text { Z } & \text { iP } & 20.5 \mathrm{c} \\ \mathrm{Ke} & \text { Z } & \text { iP } & 19.4 \mathrm{c}\end{array}$

C\&GS card 101-63:

07:51:07.9

$62.7^{\circ}$ N., $149.5^{\circ} \mathrm{W}$.

Central Alaska

$\mathrm{h}$ about $95 \mathrm{~km}$

Magnitude 5.1 (CGS).

Dec. 15

$\begin{array}{lrrr}\text { M } & \text { Z } & T \max & \text { 16:30:12 } \\ \text { A } & \text { Z } & \text { Tmax } & 14 \\ \text { D } & \text { Z } & T \max & 15 \\ \text { MP } & \text { Z } & T \text { max } & 07 \\ \text { U } & \text { Z } & T \max & 12 \\ \text { Pa } & \text { Z } & T \max & 18 \\ \text { Hi } & \text { Z } & T \max & 00 \\ \text { Ha } & \text { Z } & T \max & \text { 16:29:31 }\end{array}$

C\&GS card 102-63:

$15: 44: 22.3$

$51.0^{\circ}$ N., $128.8^{\circ} \mathrm{W}$.

Vancouver Island region

$\mathrm{h}$ about $33 \mathrm{~km}$

Magnitude 4.0 (CGS).

Dec. 15

$\begin{array}{lllc}M & Z & \text { eP } & 19: 47: 17.5 \mathrm{~d} \\ \mathrm{~A} & \mathrm{Z} & \text { eP } & 17.9 \mathrm{~d} \\ \text { U } & \text { PEZ } & \text { iS } & 19: 57: 58\end{array}$

C\&GS card 103-63:

$19: 34: 45.5$

$4.8^{\circ} \mathrm{S} ., 108.0^{\circ} \mathrm{E}$.

Java Sea 
Table 5.--Distant earthquakes--Continued

Dec. 15, 1963--Continued

\section{C\&GS card--Continued \\ $\mathrm{h}$ about $650 \mathrm{~km}$ \\ Felt: Djakarta \\ Magnitude 6.4 (CGS).}

Dec. 18

\begin{tabular}{|c|c|c|c|}
\hline M & Z & iP & $00: 38: 43.4$ \\
\hline $\mathrm{Pa}$ & Z & iP & 44.7 \\
\hline $\mathrm{Na}$ & Z & iP & 40.5 \\
\hline $\mathrm{Ka}$ & Z & eP & 47.8 \\
\hline $\mathrm{Hi}$ & Z & $\mathrm{eP}$ & 45.7 \\
\hline $\mathrm{Ha}$ & $\mathrm{Z}$ & iP & 50.1 \\
\hline $\mathrm{U}$ & PEZ & iP & 43 \\
\hline U & PEZ & iR & $00: 52: 12$ \\
\hline $\mathrm{U}$ & $\mathrm{PEE}$ & is & $00: 45: 47$ \\
\hline U & $\mathrm{PEE}$ & iSS & $00: 49: 22$ \\
\hline $\mathrm{U}$ & PEN & $i G$ & $00: 50: 00$ \\
\hline
\end{tabular}

C\&GS card 107-63:

$00: 30: 02.6$

$24.8^{\circ}$ S., $176.6^{\circ} \mathrm{W}$.

Tonga Is lands

Felt: Tonga, Fiji \& Kermadec

Is lands.

$\mathrm{h}$ about $46 \mathrm{~km}$

Magnitude 6.5 (CGS).

Dec. 20

$\begin{array}{lllr}M & Z & \text { iP } & 09: 09: 12.4 \mathrm{c} \\ \mathrm{MP} & \mathrm{Z} & \mathrm{eP} & 13.1 \mathrm{c} \\ \mathrm{Hi} & \mathrm{Z} & \mathrm{eP} & 14.0 \mathrm{c}\end{array}$

C\&GS card 103-63:

$08: 57: 17.8$

7. $0^{\circ}$ S., $129.3^{\circ} \mathrm{E}$.

Banda Sea

$h$ about $103 \mathrm{~km}$

Magnitude 5.5 (CGS).

Dec. 21

$\begin{array}{lllcc}M & \text { Z } & \text { eP } & 12: 42: 49 & d \\ \mathrm{Na} & \mathrm{Z} & \text { iP } & 47.9 & \mathrm{~d} \\ \mathrm{Hi} & \mathrm{Z} & \mathrm{eP} & 53 & \mathrm{~d}\end{array}$

C\&GS card 104-63:

$12: 34: 22.7$
Dec. 2l--Continued

$$
\begin{aligned}
& \text { C\&GS card--Continued: } \\
& 21.2^{\circ} \mathrm{S} ., 175.8^{\circ} \mathrm{W} . \\
& \text { Tonga Islands } \\
& \mathrm{h} \text { about } 90 \mathrm{~km} \\
& \text { Magnitude } 5.1 \text { (CGS). }
\end{aligned}
$$

Dec. 21

$\begin{array}{lllr}M & Z & \text { iP } & 13: 21: 18.4 \mathrm{c} \\ \mathrm{A} & \mathrm{Z} & \mathrm{eP} & 19.1 \mathrm{c} \\ \mathrm{D} & \mathrm{Z} & \text { iP } & 18.4 \mathrm{c} \\ \mathrm{MP} & \mathrm{Z} & \text { iP } & 19.7 \mathrm{c} \\ \mathrm{U} & \mathrm{Z} & \mathrm{eP} & 18.9 \mathrm{c} \\ \mathrm{Pa} & \mathrm{Z} & \text { iP } & 20.4 \mathrm{c} \\ \mathrm{Na} & \mathrm{Z} & \text { iP } & 17.8 \mathrm{c} \\ \mathrm{Ka} & \mathrm{Z} & \mathrm{eP} & 17.8 \mathrm{c} \\ \mathrm{Hi} & \mathrm{Z} & \text { iP } & 20.0 \mathrm{c}\end{array}$

C\&GS card 105-63:

13:09:09.6

$16.1^{\circ} \mathrm{N} ., 119.7^{\circ} \mathrm{E}$.

Near west coast of Luzon, P.I. $\mathrm{h}$ about $49 \mathrm{~km}$

Felt: Manila

Magnitude 5.6 (CGS).

Dec. 24

$\begin{array}{lllr}M & Z & \text { eP } & 11: 27: 17.6 \mathrm{c} \\ \mathrm{U} & \mathrm{Z} & \text { iP } & 17.2 \mathrm{c}\end{array}$

C\&GS card 105-63:

$11: 18: 15.2$

13. $1^{\circ} \mathrm{S} ., 166.7^{\circ} \mathrm{E}$.

Santa Cruz Islands region $\mathrm{h}$ about $61 \mathrm{~km}$

Magnitude 5.5 (CGS). 
Dec. 28, 1963

$\begin{array}{lllr}M & Z & \text { eP } & 05: 54: 53.0 \mathrm{c} \\ \mathrm{D} & \mathrm{Z} & \mathrm{eP} & 51.9 \mathrm{c} \\ \mathrm{Na} & \mathrm{Z} & \text { iP } & 50.4 \mathrm{~d} \\ \mathrm{Hi} & \mathrm{Z} & \text { iP } & 55.7 \mathrm{c} \\ \mathrm{Ha} & \mathrm{Z} & \mathrm{eP} & 57.8 \mathrm{c}\end{array}$

C\&GS card 105-63:

$05: 45: 20.2$

$5.1^{\circ}$ S., $153.5^{\circ} \mathrm{E}$.

New Ireland region

$\mathrm{h}$ about $70 \mathrm{~km}$

Magnitude 5.5 (CGS).

Dec. 28

$\begin{array}{lllr}M & Z & \text { iP } & 09: 13: 35.0 \mathrm{c} \\ \mathrm{A} & \mathrm{Z} & \text { iP } & 34.3 \mathrm{c} \\ \mathrm{D} & \mathrm{Z} & \text { iP } & 33.6 \mathrm{c} \\ \mathrm{MP} & \mathrm{Z} & \text { iP } & 34.6 \mathrm{c} \\ \mathrm{U} & \mathrm{Z} & \text { iP } & 34.5 \mathrm{c} \\ \mathrm{Pa} & \mathrm{Z} & \text { iP } & 35.7 \mathrm{c} \\ \mathrm{Na} & \mathrm{Z} & \text { iP } & 31.2 \mathrm{c} \\ \mathrm{Ka} & \mathrm{Z} & \text { iP } & 37.4 \mathrm{c} \\ \mathrm{Hi} & \text { Z } & \text { iP } & 37.3 \mathrm{c}\end{array}$

C\&GSS card 106-63:

09:03:52.9

$32.7^{\circ}$ S., $178.9^{\circ} \mathrm{W}$.

Kermadec Islands

$\mathrm{h}$ about $33 \mathrm{~km}$

Magnitude 5.5-5.75 ( $\mathrm{Pal}$ )

5.8 (CGS). 
The following persons or agencies reported "felt" earthquakes during the fourth quarter, 1963. Their assistance is gratefully acknowledged.

North Kona

Mr. Ready

Dr. Heather

Mr. Sherwood

Dr. Tabrah

Mrs. Tabrah

Mrs. Christensen

Mrs. Van Gorder

Hilo region

Mrs. Shaffer

Mr. Isherwood

Mrs。 Lewis

Mrs. Chiquita

Sister Thecla

Mr. Okamura

Mrs. Okamura

Mrs. Potlock

Mr. Shoemaker

Mr. Baldwin

Mr. Blackwood

Kau region

Mrs. Paiva

Mrs. Ashton

Mr. Meinecke

Mrs. Billing

Miss Yamamoto

Mrs. Walters

Puna

Mr. Warner

Mr. Williamson

Miss Takemoto
Kilauea summit region

Mr. Smith

Mr. Bush

Mr. Forbes

Mrs. Yong

$\mathrm{Mr}$. Yong

Mrs. Mist

Mrs. Wentworth

Mrs. Yamamoto

Miss English

Mrs. Hansen

Mrs. Duncan

$\underline{\text { Kona coast }}$

Mrs. Glass

Mrs. Higashihara

Mr. Ladd

Miss Greenwell

Mr. Johnston

Mr. Apple

Mr. Yeaman

Mr. Wallace

Mr. Sutherland

Mr. Sleightholm

Mrs. Gray

Central Hawaii

Mrs. Vredenburg

Mrs. Lindsey 


\section{Publications of special interest and HVO \\ numbered contributions, 1963}

Kinoshita, W. T., Krivoy, H. L., Mabey, D. R., and McDonald, R. R., 1963, Gravity survey of the is.land of Hawaii: U.S. Geol. Survey Prof. Paper 475-C, art. 89, p. 114-116. (HVO Contr. 182)

Moore, J. G., and Reed, D. K., 1963, Pillow structures of submarine basalts east of Hawaii: U.S. Geol. Survey Prof. Paper 475-B, art. 40, p. 153-157. (HVO Contr. 181)

Muir, I. D., and Tilley, C. E., 1963, Contributions to the petrology of Hawaiian basalts, II, The tholeiitic basalts of Mauna Loa and Kilauea: Am. Jour. Sci., v. 261, no. 2, p.111-128.

Richter, D. H., 1963, Volcano observations: Am. Geophys. Union Trans., v. 44, no. 2, p. 505-507. (HVo Contr. 180) 